\title{
Core Movement During Processing of Sandwich Panels
}

\author{
by
}

\author{
Duncan Joseph Pawson
}

B.Sc. Mechanical Engineering, University of Calgary, 2015

\begin{abstract}
A THESIS SUBMITTED IN PARTIAL FULFILLMENT
OF THE REQUIREMENTS FOR THE DEGREE OF
\end{abstract}

\section{Master of Applied Science}

in

THE FACULTY OF GRADUATE AND POSTDOCTORAL STUDIES

(Materials Engineering)

The University of British Columbia

(Vancouver)

August 2019

(C) Duncan Joseph Pawson, 2019 
The following individuals certify that they have read, and recommend to the Faculty of Graduate and Postdoctoral Studies for acceptance, the thesis entitled:

\section{Core Movement During Processing of Sandwich Panels}

submitted by Duncan Joseph Pawson in partial fulfillment of the requirements for the degree of Master of Applied Science in Materials Engineering.

\section{Examining Committee:}

Göran Fernlund, Materials Engineering

Supervisor

Anoush Poursartip, Materials Engineering

Supervisory Committee Member

Chad Sinclair, Materials Enginieering

Additional Examiner 


\section{Abstract}

Core movement is a phenomenon that plagues sandwich panel fabrication during autoclave processing. External pressure can result in deformation of the core in its weak, lateral direction. This in turn, drags the facesheet plies inward. Parts displaying notable core movement are unfit for service and must be scrapped. Restraining plies, thereby preventing core movement, has become common practice in industrial processing of sandwich panels. Progress has been made in mitigating the risk of core movement through artificially increasing pressure within the core as well as through development of high friction resin/fiber systems. However, a complete understanding of the physics of the problem is still lacking.

The focus of this research is to understand how processing conditions affect core movement and to gain a better understanding of the fundamental nature of core movement. Parameters investigated include, temperature, pressure, and a variety of structural features such as tie downs, core machining stabilization, and core chamfer angles. The problem is investigated using novel techniques. In-situ data collection is performed through use of displacement and in-core pressure sensors. Moreover, the entire event is filmed in real time using an autoclavable camera. This allowed for identification of the exact processing parameters during initiation and progression of core movement. Individual ply movement is determined post-processing. It was shown that failure pressure is dictated by viscosity at lower processing temperatures, but at high temperatures this is no longer the case. This implies a shift in the lubrication regime and alters the mechanics of the problem.

A basic mechanical model describing the core movement process is outlined and the conditions necessary for core movement initiation are proposed. It was shown that core movement initiates at the chamfer radius and fluctuates between progressing through the core and down the chamfer edge. The results suggest that friction is the governing mechanism behind core movement initiation, which, if true, has important ramifications for sandwich panel design.

This work builds off previous research and lays the foundations for future work. 


\section{Lay Summary}

Composite sandwich panels are comprised of a low density core material surrounded by stiff, composite facesheets. They are important for structural engineering applications, particularly in aerospace. They are typically processed in industrial-scale autoclaves, which apply temperature and pressure to the part. Unfortunately, the external pressure can often cause the core material to collapse inward; known as core movement. This body of work investigates the basic parameters that influence core movement and relates this to the underlying physics of the phenomenon. Novel techniques are implemented, wherein the core movement process is measured in-situ through use of embedded sensors and a video camera. This allows for an accurate understanding of the problem and the conditions necessary to initiate core movement. 


\section{Preface}

This dissertation titled "Core Movement During Processing of Sandwich Panels" is original work conducted and written by Duncan Pawson under the supervision of Professor Göran Fernlund.

All experiments and analysis outlined in Chapter 4, Chapter 5, and Chapter 6 were designed and performed by Duncan Pawson while under the supervision of Professor Göran Fernlund. The aims and objectives described in Chapter 3 as well as Figure 3.1 were developed by Duncan Pawson while under the supervision of Professor Göran Fernlund. Each chapter was written by Duncan Pawson under the supervision of Professor Göran Fernlund. Materials for the research were provided by Mark Shead at Boeing Canada - Winnipeg. The sensors and autoclavable camera were provided by Convergent Manufacturing Technologies.

Results from Section 5.1 were presented at the $75^{\text {th }}$ International Society for the Advancement of Material and Process Engineering (SAMPE) in Charlotte, North Carolina, in May 2019. A conference paper was published in the proceedings with the following details. Pawson, Duncan and Fernlund, Göran. (2019). "Core Movement During Processing of Sandwich Panels", Proceedings of the Society for the Advancement of Material and Process Engineering - Seventy-fifth Technical Conference, Charlotte, 2019. The paper was written by Duncan Pawson under the supervision of Professor Göran Fernlund. The presentation was given by Duncan Pawson.

Results from Section 5.3 were presented at the $11^{\text {th }}$ Canadian - International Conference on Composites (CANCOM) in Kelowna, British Columbia, in July, 2019. A conference paper was published in the proceedings with the title, "Layup effects on core movement in sandwich panels". The listed authors are Duncan Pawson and Göran Fernlund. The conference paper was written by Duncan Pawson under the supervision of Professor Göran Fernlund. The presentation was given by Duncan Pawson. 


\section{Table of Contents}

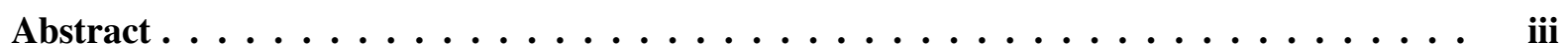

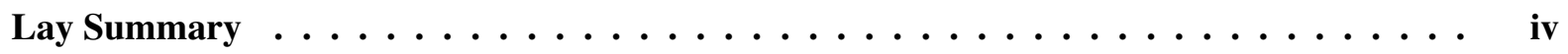

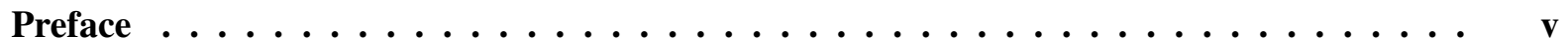

Table of Contents $\ldots \ldots \ldots \ldots \ldots \ldots \ldots \ldots \ldots$

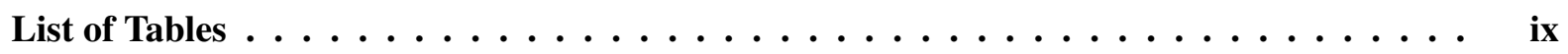

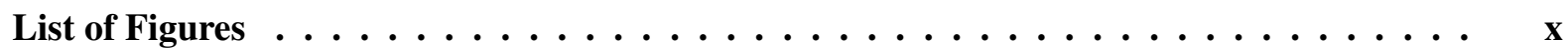

List of Symbols $\ldots \ldots \ldots \ldots \ldots \ldots \ldots \ldots \ldots \ldots \ldots \ldots \ldots \ldots$

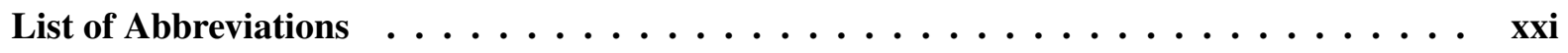

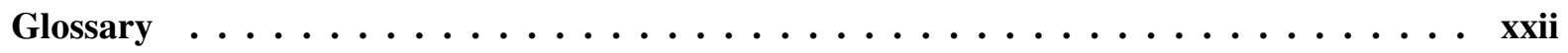

Acknowledgments $\ldots \ldots \ldots \ldots \ldots \ldots \ldots \ldots \ldots \ldots \ldots \ldots \ldots \ldots \ldots$

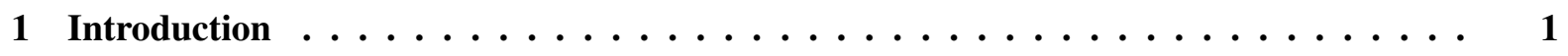

1.1 Composites and sandwich panels . . . . . . . . . . . . . . 1

1.2 Core movement . . . . . . . . . . . . . . . . 2

2 Literature Review .............................. 5

2.1 Honeycomb mechanics . . . . . . . . . . . . . . . . . 5

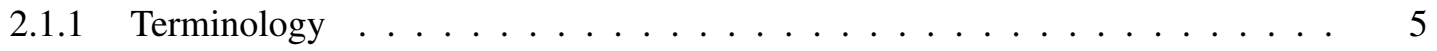

2.1 .2 Relative density . . . . . . . . . . . . . . . . 7

2.1 .3 Uniaxial loading . . . . . . . . . . . . . . . . . 7

2.1 .4 Linear elastic bending . . . . . . . . . . . . . . . 9

2.1 .5 Cell collapse ........................... 10

2.1.6 Biaxial loading . . . . . . . . . . . . . . . . . 11

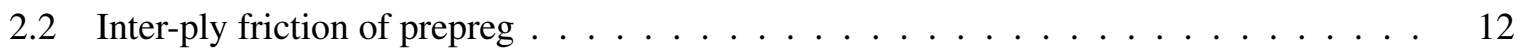

2.2.1 Pre-yield behaviour . . . . . . . . . . . . . . . 13 
2.3 Core Movement . . . . . . . . . . . . . . . . . . . . . . . 14

2.3.1 Physics of core movement . . . . . . . . . . . . . . 14

2.3 .2 Mitigation methods . . . . . . . . . . . . . . . . 16

2.3.3 Gas flow within the core . . . . . . . . . . . . . 18

3 Aims and Objectives . . . . . . . . . . . . . . . . 20

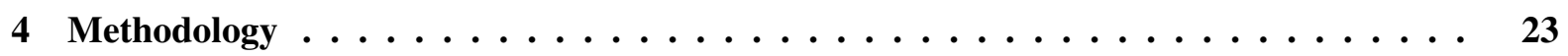

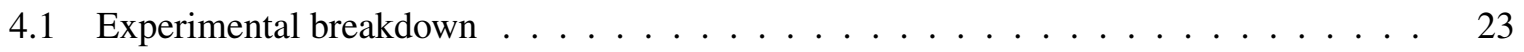

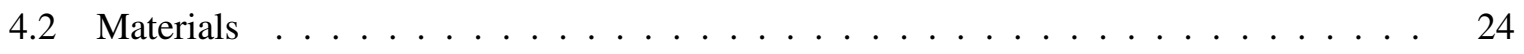

4.3 Sensors . . . . . . . . . . . . . . . . . . . . 24

4.4 Layup . . . . . . . . . . . . . . . . . . . . . . . . 26

4.4 .1 Standard layup . . . . . . . . . . . . . . . . . 26

4.4 .2 Specific layups . . . . . . . . . . . . . . . . . 27

4.5 Cure cycle . . . . . . . . . . . . . . . . . . . . . . . 28

4.5.1 Standard cure cycle . . . . . . . . . . . . . . . . . . 29

4.5.2 Specific cure cycles . . . . . . . . . . . . . . . . . . . 29

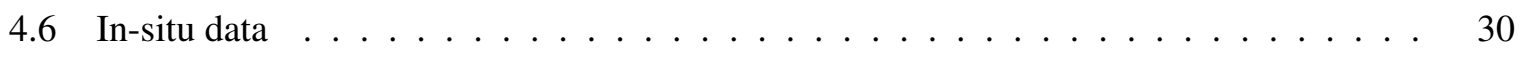

4.7 Post cure data . . . . . . . . . . . . . . . . . . . 32

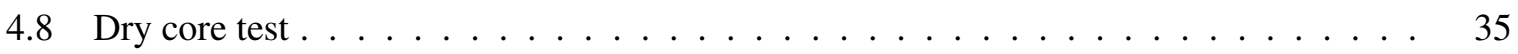

5 Results and Discussion . . . . . . . . . . . . . . . . . 36

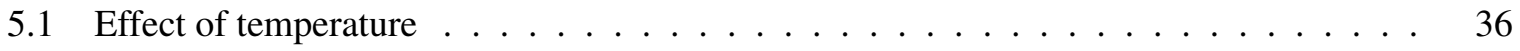

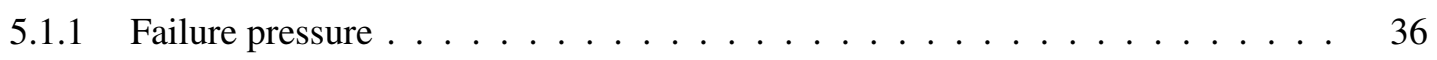

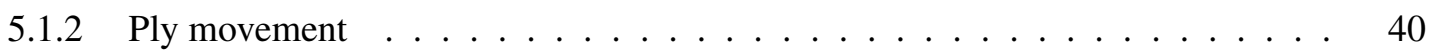

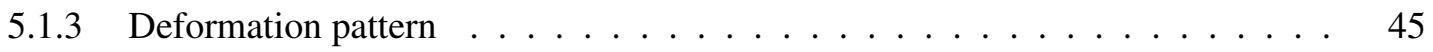

5.2 Effect of pressure . . . . . . . . . . . . . . . . . 49

$5.2 .1 \quad$ Failure temperature . . . . . . . . . . . . . . . . . . . 49

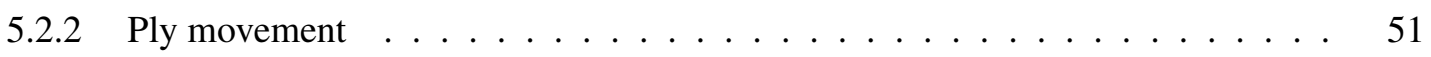

5.2 .3 Extent of core movement . . . . . . . . . . . . . . 54

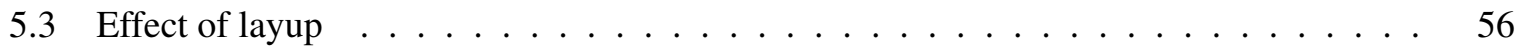

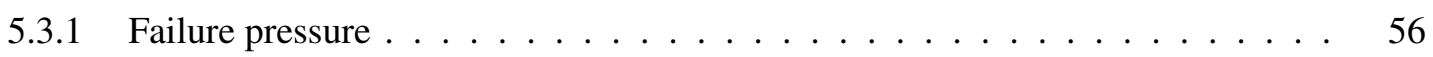

5.3 .2 Ply movement $\ldots \ldots \ldots \ldots \ldots \ldots$

5.3 .3 Extent of core movement . . . . . . . . . . . . . . 61

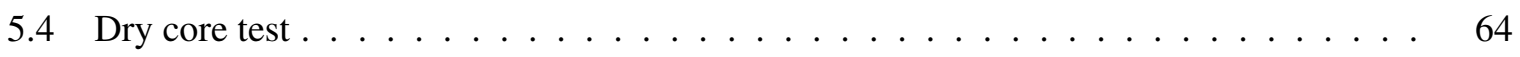

$5.4 .1 \quad$ Failure pressure . . . . . . . . . . . . . . . 64

5.5 Core movement mechanics . . . . . . . . . . . . . . . . . 6 68

5.5.1 Initiation of core movement . . . . . . . . . . . . 68

5.5.2 Progression of core movement . . . . . . . . . . . . . 69

5.5 .3 In-core pressure . . . . . . . . . . . . . . 89 
6 Summary and Improved Model . . . . . . . . . . . . . . . . . . . . 92

6.1 Drawback of current models . . . . . . . . . . . . . . . . . . . . 92

6.2 Core movement process . . . . . . . . . . . . . . . . . . . . 97

6.2 .1 Conditions for initiation . . . . . . . . . . . . . . . 97

6.2 .2 Conditions for progression . . . . . . . . . . . . . . . . 101

6.3 Processing window . . . . . . . . . . . . . . . . . . 103

6.4 Design improvements . . . . . . . . . . . . . . . . . . . . 108

6.4 .1 Recommendations . . . . . . . . . . . . . . . . . . 110

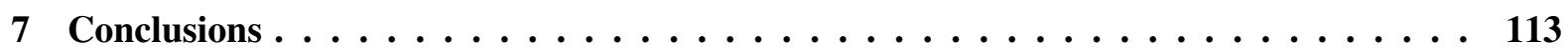

7.1 Summary of results . . . . . . . . . . . . . . . . . . . 113

7.2 Future work . . . . . . . . . . . . . . . . . 115

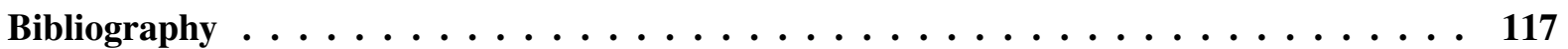

Appendix A Data Acquisition . . . . . . . . . . . . . . . . . 121

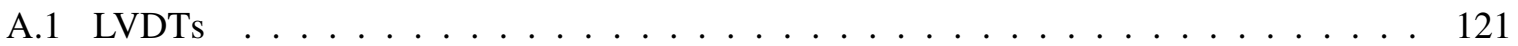

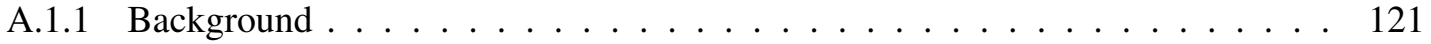

A.1.2 AC conditioner calibration . . . . . . . . . . . . . . 121

A.2 In-core pressure sensors . . . . . . . . . . . . . . . . . . . 123

A.3 Autoclavable camera . . . . . . . . . . . . . . . . . . 123

Appendix B Additional Post-processing Images . . . . . . . . . . . . . . . . . . . . . 124

B.1 Post-processed panels . . . . . . . . . . . . . . . . . . . . . . . . . . . . . . . 124

B.2 Slope gradient maps . . . . . . . . . . . . . . . . . . . . 125

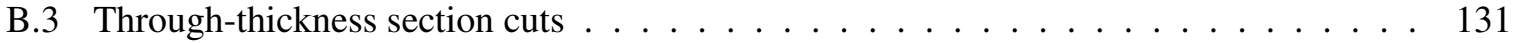

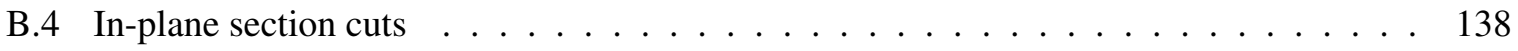

Appendix C Additional Data Sets . . . . . . . . . . . . . . . . . 147

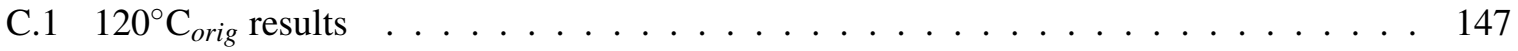




\section{List of Tables}

Table 4.1 Summary of experiments. Note that the $120^{\circ} \mathrm{C}$ and "Standard" experiment are the same test and represent the baseline test. . . . . . . . . . . . . . . . .

Table 5.1 Extent of deformation in the ribbon and non-ribbon direction associated with different processing temperatures. . . . . . . . . . . . . . . .

Table 5.2 Difference in displacement between the ribbon and non-ribbon edges associated with different processing temperatures. . . . . . . . . . . . . . . .

Table 5.3 Extent of deformation in the ribbon and non-ribbon direction associated with different processing pressures. . . . . . . . . . . . . . . . .

Table 5.4 Extent of deformation in the ribbon and non-ribbon direction associated with various layup features. . . . . . . . . . . . . . . . . . .

Table 5.5 Average panel height within the crush zone compared with the degree of core movement in the L direction. . . . . . . . . . . . . . . . . . .

Table 5.6 Average panel height within the crush zone compared with the degree of core movement in the $\mathrm{W}$ direction. . . . . . . . . . . . . . . . . . . . 86

Table 5.7 In-core pressure at onset and collapse. . . . . . . . . . . . . . . 89

Table 6.1 Main parameters involved in the initiation of core movement. . . . . . . . . . . 109 


\section{List of Figures}

Figure 1.1 (A) Sandwich panel after layup and prior to cure. (B) Same panel following cure; drastic core movement has occured. . . . . . . . . . . . . . . .

Figure 1.2 Processed sandwich panel on tool. Grit strips surrounding the part are used to restrain plies (tie-downs). Indents in the panel are from pressure sensors. . . . . . . .

Figure 2.1 Hexagonal honeycomb structure. Glue lines are present in the ribbon direction (L) as indicated by the red lines. The non-ribbon direction $(\mathrm{W})$ is perpendicular in-plane to this. . . . . . . . . . . . . . . . . . . . . . . . .

Figure 2.2 Honeycomb unit cell, where $\ell$ represents length, $h$ height, $t$ thickness, $c$ cell size, $\alpha$ the angle between each side, and $\theta$ the cell wall angle. For regular honeycomb structures $h=\ell, \alpha=120^{\circ}$, and $\theta=30^{\circ}$. The out-of-plane thickness is denoted as $b$.

Figure 2.3 Typical compressive stress-strain curve for an elastomeric honeycomb. I: Bending linear elastic bending of cell walls, II: Collapse (plateau region) - constant stress as cells fail, III: Densification - cell walls touching. Figure adapted from [22]. . . . .

Figure 2.4 Effect of increasing nominal relative density on the compressive stress-strain response of honeycombs. I: Bending - linear elastic bending of cell walls, II: Collapse (plateau region) - constant stress as cells fail, III: Densification - cell walls touching. Figure adapted from $[22] . \ldots \ldots \ldots \ldots \ldots$

Figure 2.5 Stribeck Curve demonstrating three regions of lubrication. I: Boundary lubrication, II: Mixed lubrication, III: Hydrodynamic lubrication. Figure adapted from [34]. . .

Figure 2.6 FBD of sandwich panel demonstrating forces involved in core movement. $F_{\text {stiffness }}$ includes both the material stiffness of the skins and core in the lateral direction as well as the in-core pressure. Figure adapted from $[28] \ldots \ldots$. . . . . . . . . . .

Figure 3.1 Core movement map. Major factors known to influence core movement are displayed. Green arrows indicate when one property influences another. Properties with an asterisk are suspected to influence core movement, but their relation to core movement has not yet been documented nor are they investigated in this body of work. 21 
Figure 4.1 Test setup showing in-situ sensors. Top left is an LVDT sensor, top right is an in-core pressure sensor, and the bottom is the full setup in the autoclave with the camera mounted above the panel. . . . . . . . . . . . . . . .

Figure 4.2 Standard sandwich panel layup. First layer against the tool is surfacing film, followed by 4 toolside plies. Core with pressure sensor and film adhesive layers is then added, followed by 4 filler plies. Next, 4 bagside plies are draped over the core. PTFE release film, breather, and vacuum bagging are then added. The Linear Variable Displacement Transducers (LVDTs) are placed after vacuum bagging is

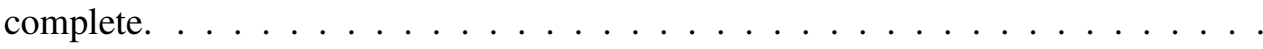

Figure 4.3 Core machining stabilization - $76.2 \mathrm{~mm}$ wide picture frame film adhesive strip is cured along the toolside of the core. (A) Toolside, (B) Bagside. . . . . . . . . . . .

Figure 4.4 Representative viscosity profile for three different temperatures during cure. Adapted from RAVEN data received from M. Shead (Boeing Winnipeg). . . . . . . . . . .

Figure 4.5 Data output from in-situ sensors. The colour scheme is as follows: black - autoclave pressure, blue - LVDT sensor, purple - in-core pressure sensor, green - vacuum pressure. The region between onset and collapse represents the critical zone, wherein core movement initiates. . . . . . . . . . . . . .

Figure 4.6 Polished through-thickness section cut. The region between the panel edge and core edge is referred to as the laminate edge. . . . . . . . . . . . . . . . . . . .

Figure 4.7 Cross sectional image of laminate edge showing individual plies and where they terminate after core movement. Numbers on the right identify the individual plies while the red Xs indicate where the associated ply terminates. . . . . . . . . .

Figure 4.8 Polished in-plane section cut showing the deformation profiles in L and W. . . . .

Figure 5.1 Net pressure acting on the sandwich panels at onset and collapse for the given (a) processing temperature and (b) associated resin viscosity. . . . . . . . . . .

Figure $5.225^{\circ} \mathrm{C}, 95^{\circ} \mathrm{C}, 120^{\circ} \mathrm{C}$, and $180^{\circ} \mathrm{C}$ tests post-processing, showing the extent of core

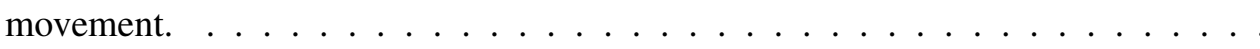

Figure 5.3 Schematic showing the expected thickness changes along the laminate edge of a sandwich panel that (a) experiences no core movement and (b) experiences core

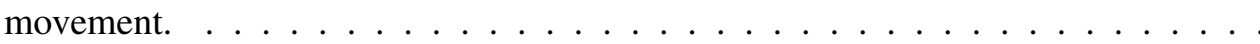

Figure 5.4 Change in thickness across laminate edge of processed panels in the L and W directions at varying temperatures. The "No movement" case represents the test with tie down plies, as no visible core movement was seen. . . . . . . . . . . . .

Figure 5.5 Microscope image of laminate edge showing surfacing film seeping through interstitial zones between tows. . . . . . . . . . . . . . . . .

Figure 5.6 Specific ply movement profile of processed panels in $\mathrm{L}$ and $\mathrm{W}$ at varying temperatures. Initially, the core edge is $50.8 \mathrm{~mm}$ from the panel edge. . . . . . . . . 
Figure 5.7 Core movement in ribbon and non-ribbon directions for (a) low versus (b) high temperature processing. Autoclave pressure is absolute external pressure. Vacuum

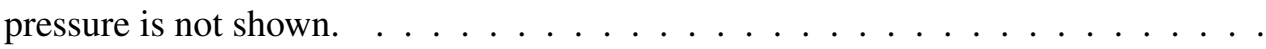

Figure 5.8 (a) Temperature at which onset and collapse occur for sandwich panels processed at a specific pressure and (b) the associated resin viscosity at failure. . . . . . . . . .

Figure 5.9 Change in thickness across laminate edge of processed panels in the $\mathrm{L}$ and $\mathrm{W}$ directions at varying pressures. The "No movement" case represents the test with tie down plies, as no visible core movement was seen. . . . . . . . . . . . . . . .

Figure 5.10 Specific ply movement profile of processed panels in $\mathrm{L}$ and $\mathrm{W}$ at varying pressures. Initially, the core edge is $50.8 \mathrm{~mm}$ from the panel edge. . . . . . . . . 53

Figure 5.11325 and $600 \mathrm{kPa}$ tests post-processing, showing the extent of core movement. . . .

Figure 5.12 Core movement in ribbon and non-ribbon direction for high pressure $(600 \mathrm{kPa})$ processing. Autoclave pressure is absolute external pressure. Vacuum pressure is

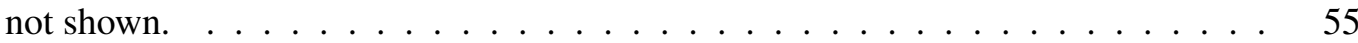

Figure 5.13 Minor deformations in cellular pattern of full tie down test. . . . . . . . . . 56

Figure 5.14 Net pressure acting on the sandwich panels at onset and collapse for varying layups. The data for half tie downs represents that of the unrestrained edges. . . . . . . . .

Figure 5.15 In-situ data from $45^{\circ}$ core test. Collapse occurs immediately after venting of vacuum, following a one minute hold at higher pressure. . . . . . . . . . . . .

Figure 5.16 Change in thickness across laminate edge of processed panels in the L and $\mathrm{W}$ directions for varying layups. The full tie down cases show no externally visible core movement.

Figure 5.17 Specific ply movement profile of processed panels in $\mathrm{L}$ and $\mathrm{W}$ for varying layups. Initially, the core edge is $50.8 \mathrm{~mm}$ from the panel edge . . . . . . . . . .

Figure 5.18 Microscope images showing termination of plies 5-6 (red circle), ply 7 (yellow circle), and ply 8 (blue circle) in the $\mathrm{W}$ and $\mathrm{L}$ directions for the Core Machining Stabilization $(\mathrm{CMS})$ sample. . . . . . . . . . . . . . . . . . .

Figure 5.19 Microscope images showing termination of plies 5-6 (red circle), ply 7 (yellow circle), and ply 8 (blue circle) in the $\mathrm{W}$ and $\mathrm{L}$ directions for the $45^{\circ}$ chamfer sample.

Figure 5.20 Standard, full tie down, half tie down, CMS, and $45^{\circ}$ chamfer tests post-processing, showing extent of core movement. . . . . . . . . . . . .

Figure 5.21 In-situ results from dry core test. Cells deform upon loading and recover almost entirely upon unloading. . . . . . . . . . . . . . . . . .

Figure 5.22 Snapshots taken during autoclave test for dry honeycomb. 1: Prior to pressure application. 2: Moment of collapse. 3: Maximum deformation. 4: Fully unloaded honeycomb core following depressurization. . . . . . . . . . . .

Figure 5.23 Plastic deformation in $\mathrm{W}$ following autoclave pressurization. (A) Image of entire honeycomb. (B) Magnified view of deformation in W. . . . . . . . . . . . . 
Figure 5.24 Change in surface roughness from onset of core movement through to collapse. Transitioning from 1-4, a notable change in surface roughness is seen within the green circle. The bagside plies conform to the honeycomb structure as it deforms, observable through the breather cloth. . . . . . . . . . . . .

Figure 5.25 In-plane initiation of core movement showing the progression of cell bending throughout the honeycomb. Images are from different tests showing (a) no core movement, (b) minor bending, (c) widespread bending, and (d) early stages of collapse. . . . .

Figure 5.26 Slope angle of processed panels. Dark red indicates a vertical slope with an angle of $90^{\circ}$. Dark blue indicates a flat plane with an angle of $0^{\circ}$. For most samples, a slope angle greater than $20^{\circ}$ indicates crush has occurred along the chamfer edge. For the $45^{\circ}$ chamfer, a slope angle larger than $45^{\circ}$ indicates crush. . . . . . . . . .

Figure 5.27 (a) Standard and (b) CMS crush patterns, displaying growth of the crush zone from image 1-4. Red arrows show the crush front in the L direction while blue arrows show the crush front in the $\mathrm{W}$ direction. Elapsed time from the application of pressure is displayed. Note the change in orientation between samples. . . . . . . . . .

Figure 5.28 Crush pattern for $600 \mathrm{kPa}$ sample, displaying growth of the crush zone from image 1-4. W crush front (blue arrow) progresses at a faster rate than the $\mathrm{L}$ crush front (red arrow). Elapsed time from the application of temperature is displayed. . . . .

Figure 5.29 Rate of deformation of the top and bottom of a $20^{\circ}$ chamfer edge witnessed through (a) in-situ sensors and (b) in-situ camera footage. Red arrows points to the bottom of the chamfer edge while yellow arrows points to the top. Each square in (b) is $12.7 \times 12.7 \mathrm{~mm}$, allowing for the crush rate to be determined. . . . . . . . . . .

Figure 5.30 Rate of deformation of the top and bottom of a $45^{\circ}$ chamfer edge witnessed through (a) in-situ sensors and (b) in-situ camera footage. Red arrows points to the bottom of the chamfer edge while orange arrows points to the top. Each square in (b) is $12.7 \times 12.7 \mathrm{~mm}$, allowing for the crush rate to be determined. . . . . . . . . .

Figure 5.31 Schematic showing the manner of crush progression through the core. A: Crush initiates at chamfer radius and progresses inward - bottom and top of chamfer move at same rate. B: Crush initiates at chamfer radius and progresses down chamfer edge - bottom of chamfer displaces quicker than top creating a concave surface. $\mathrm{C}$ : Actual manner of crush progression through core - combination of A-B-A. 1 - Crush initiates at chamfer radius and progresses inward (similar to A). 2 - Chamfer edge crushes quicker than cells beyond radius (similar to B). 3 - Crush switches again to collapse cells further within core $($ similar to $\mathrm{A}) \ldots \ldots \ldots \ldots$

Figure 5.32 Through-thickness section cuts of processed samples. Purple indicates the crush zone, blue the densified regions, and red the bagside wrinkles. Additional features are outlined in black. (a) The $120^{\circ} \mathrm{C}$, standard layup and (b) 45 chamfer samples are provided here. Other samples are shown in the following images. . . . . . . . 79

Figure 5.33 (a) CMS and (b) half tie-down samples. . . . . . . . . . . . . . 80 
Figure 5.34 Full tie-down and room temperature samples. . . . . . . . . . . .

Figure 5.35 General crush pattern through panel in the $\mathrm{W}$ direction. Image is taken from the $120^{\circ} \mathrm{C}$, standard layup experiment. Purple indicates the crush zone, red the bagside wrinkles, and blue the densified regions. . . . . . . . . . . .

Figure 5.36 Buckling of plies nearest the core (blue arrows) on the (a) bagside and (b) toolside surfaces within the crush zone. . . . . . . . . . . . .

Figure 5.37 Map of panel height extracted from Coordinate Measuring Machine (CMM) data for (a) no core movement and (b) drastic core movement. White bars in (b) represent the length of the crush zone in $\mathrm{L}$ and $\mathrm{W}$. There is a notable increase in height along the crush zone. . . . . . . . . . . . . . . . . . . . . . .

Figure 5.38 In-plane section cuts of processed panels showing densification along the $\mathrm{L}$ and $\mathrm{W}$ directions. The orange line represents the $\mathrm{L}$ crush front whereas the red line represents the $\mathrm{W}$ crush front. Yellow arrows point to regions of low densification in the non-ribbon direction $\ldots \ldots \ldots \ldots \ldots$

Figure 5.39 In-core pressure sensor response to core movement taken from the standard, $95^{\circ} \mathrm{C}$ sample. Pressure recovery is captured upon release of vacuum. No difference in in-core pressure is observed between onset and collapse. Following collapse, considerable noise is seen due to pulling of the sensor wires. . . . . . . . . .

Figure 6.1 Schematic showing the tension $\left(\mathrm{F}_{1}-\mathrm{F}_{4}\right)$ developed in bagside plies as core deforms. Friction $\left(\mathrm{F}_{12}-\mathrm{F}_{\text {Bag }}\right)$ at the interfaces is provided. The plies can be approximated as springs slipping relative to one another. . . . . . . . . . .

Figure 6.2 Schematic showing the first zone of resistance against core movement (i.e. the chamfer radius). In order for the cells to collapse, they must either slide relative to the under and overlying plies or the plies must buckle with the cells. . . . . . . . .

Figure 6.3 Schematic showing the second zone of resistance against core movement (i.e. the laminate edge). In order for the plies to move, they must either stretch and/or slip across an interface. . . . . . . . . . . . . . . . .

Figure 6.4 Core movement initiation for standard and high temperature processing. Each model incorporates the use of bagside Polytetrafluoroethylene (PTFE) release film and a tacky surfacing film across the tool. . . . . . . . . . . . .

Figure 6.5 Schematic showing friction across the core-ply interface. . . . . . . . . . . .

Figure 6.6 Empirical failure envelope outlining the processing conditions necessary for core movement. The curves are based on experimental results for a chamfer angle of $20^{\circ}$. Dotted lines are assumed values. Additional experimental results concerning a change in layup are provided at $120^{\circ} \mathrm{C} \ldots \ldots \ldots \ldots$

Figure 6.7 Expected failure envelope for a chamfer angle of $45^{\circ}$. The experimental results at $120^{\circ} \mathrm{C}$ are also provided. . . . . . . . . . . . . . . .

Figure 6.8 (a-b) General evolution of resistive forces with temperature and (c) Degree of Cure (DOC) . . . . . . . . . . . . . . . . . . . . . 
Figure 6.9 Effect of altering the laminate edge length on prepreg-prepreg friction at $120^{\circ} \mathrm{C}$. Red dots represent core movement initiation. In-core pressure is assumed to be equal to $1 \mathrm{~atm}$.

Figure 6.10 Effect of increasing the (a) core height and (b) chamfer angle for various laminate edge lengths at $120^{\circ} \mathrm{C}$. In-core pressure is assumed to be $1 \mathrm{~atm}$.

Figure A.1 LVDT wiring to conditioner module. Taken from [14] . . . . . . . . . . . . . 122

Figure A.2 Video footage synchronized with in-situ sensor data. $95^{\circ} \mathrm{C}$ experiment. . . . . . 123

Figure B.1 Top-down view of processed panels. . . . . . . . . . . . . . . . . . . . 125

Figure B.2 Slope gradient map for $95^{\circ} \mathrm{C}$ sample. Although not shown, the colour bar applies to each of the following images. Noise is seen in some of the samples which can be disregarded . . . . . . . . . . . . . . . . . . . 126

Figure B.3 Slope gradient map for $120^{\circ} C_{\text {orig }}$ sample . . . . . . . . . . . . . . . 126

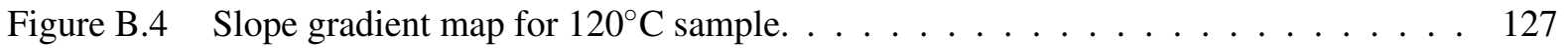

Figure B.5 Slope gradient map for $180^{\circ} \mathrm{C}$ sample. . . . . . . . . . . . . . . . . . . 127

Figure B.6 Slope gradient map for $325 \mathrm{kPa}$ sample. . . . . . . . . . . . . . . . . . . . 128

Figure B.7 Slope gradient map for $600 \mathrm{kPa}$ sample. . . . . . . . . . . . . . . . . . . . . . . . . . . . . . . . . . . . .

Figure B.8 Slope gradient map for full tie-down sample. . . . . . . . . . . . . . . . . . . . . . 129

Figure B.9 Slope gradient map for half tie-down sample. . . . . . . . . . . . . . . . . . . . 129

Figure B.10 Slope gradient map for CMS sample. . . . . . . . . . . . . . . . . . . 130

Figure B.11 Slope gradient map for $45^{\circ}$ chamfer sample . . . . . . . . . . . . . . . . 130

Figure B.12 Through-thickness section cut of room temperature sample. . . . . . . . . . 132

Figure B.13 Through-thickness section cut of $95^{\circ} \mathrm{C}$ sample. . . . . . . . . . . . . . . . . 132

Figure B.14 Through-thickness section cut of $120^{\circ} \mathrm{C}_{\text {orig }}$ sample. . . . . . . . . . . . . 133

Figure B.15 Through-thickness section cut of $120^{\circ} \mathrm{C}$ sample. . . . . . . . . . . . 133

Figure B.16 Through-thickness section cut of $180^{\circ} \mathrm{C}$ sample. . . . . . . . . . . . . . . 134

Figure B.17 Through-thickness section cut of $325 \mathrm{kPa}$ sample. . . . . . . . . . . . . . . . 134

Figure B.18 Through-thickness section cut of $600 \mathrm{kPa}$ sample. . . . . . . . . . . . . . . 135

Figure B.19 Through-thickness section cut of full tie-down sample. . . . . . . . . . . . . 135

Figure B.20 Through-thickness section cut of half tie-down sample . . . . . . . . . . . . 136

Figure B.21 Through-thickness section cut of CMS sample. . . . . . . . . . . . . . . . 136

Figure B.22 Through-thickness section cut of $45^{\circ}$ chamfer sample. . . . . . . . . . . . . 137

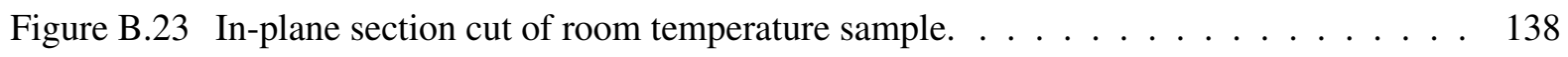

Figure B.24 In-plane section cut of $120^{\circ} \mathrm{C}$ sample. . . . . . . . . . . . . . . . . . . . 139

Figure B.25 In-plane section cut of $180^{\circ} \mathrm{C}$ sample. . . . . . . . . . . . . . . . . . 140

Figure B.26 In-plane section cut of $600 \mathrm{kPa}$ sample. . . . . . . . . . . . . . . . . . 141

Figure B.27 In-plane section cut of full tie-down sample. . . . . . . . . . . . . . . . 142

Figure B.28 In-plane section cut of half tie-down sample between two restrained edges. . . . . 143

Figure B.29 In-plane section cut of half tie-down sample between two unrestrained edges. . . . 144 
Figure B.30 In-plane section cut of CMS sample . . . . . . . . . . . . . . . . . 145

Figure B.31 In-plane section cut of $45^{\circ}$ sample. . . . . . . . . . . . . . . . . 146

Figure C.1 Core movement in ribbon and non-ribbon direction for $120^{\circ} \mathrm{C}_{\text {orig }}$ sample. Autoclave pressure is absolute external pressure. Vacuum pressure is not shown. . . . . . . . 147 


\title{
List of Symbols
}

\author{
$A_{\text {core }} \quad$ Area of core affected by lateral component of pressure $\left[\mathrm{m}^{2}\right]$. \\ $\alpha \quad$ Angle between honeycomb cell members $\ell$ and $h$ [rad]. \\ $b \quad$ Out-of-plane thickness of honeycomb cell [m]. \\ $c \quad$ Honeycomb cell size $[\mathrm{m}]$. \\ $d \quad$ Film thickness [m]. \\ $\frac{d \gamma}{d t} \quad$ Shear strain rate $\left[\mathrm{s}^{-1}\right]$. \\ E In-plane Young's modulus of regular honeycomb [Pa]. \\ $E_{1} \quad$ Young's modulus of honeycomb in $X_{1}$ direction $[\mathrm{Pa}]$. \\ $E_{2} \quad$ Young's modulus of honeycomb in $X_{2}$ direction [Pa]. \\ $E_{s} \quad$ Young's modulus of solid material from which honeycomb walls are comprised [Pa]. \\ $E_{p l y} \quad$ Young's modulus of ply [Pa]. \\ $\left(\varepsilon_{e l}^{*}\right)_{2} \quad$ Strain at cell collapse under elastic buckling. \\ $\varepsilon \quad$ In-plane compressive strain for regular honeycomb. \\ $\eta \quad$ Dynamic viscosity $[\mathrm{Pa} \cdot \mathrm{s}]$. \\ $F_{f} \quad$ Resistive force due to core material [N]. \\ $F_{f} \quad$ Coloumb friction force $[\mathrm{N}]$. \\ $F_{g} \quad$ Force due to gravity (weight) $[\mathrm{N}]$. \\ $F_{p-b} \quad$ Friction force between prepreg and bag [N]. \\ $F_{p-c} \quad$ Friction force between prepreg and core [N]. \\ $F_{p-p} \quad$ Friction force between prepreg and prepreg [N].
}




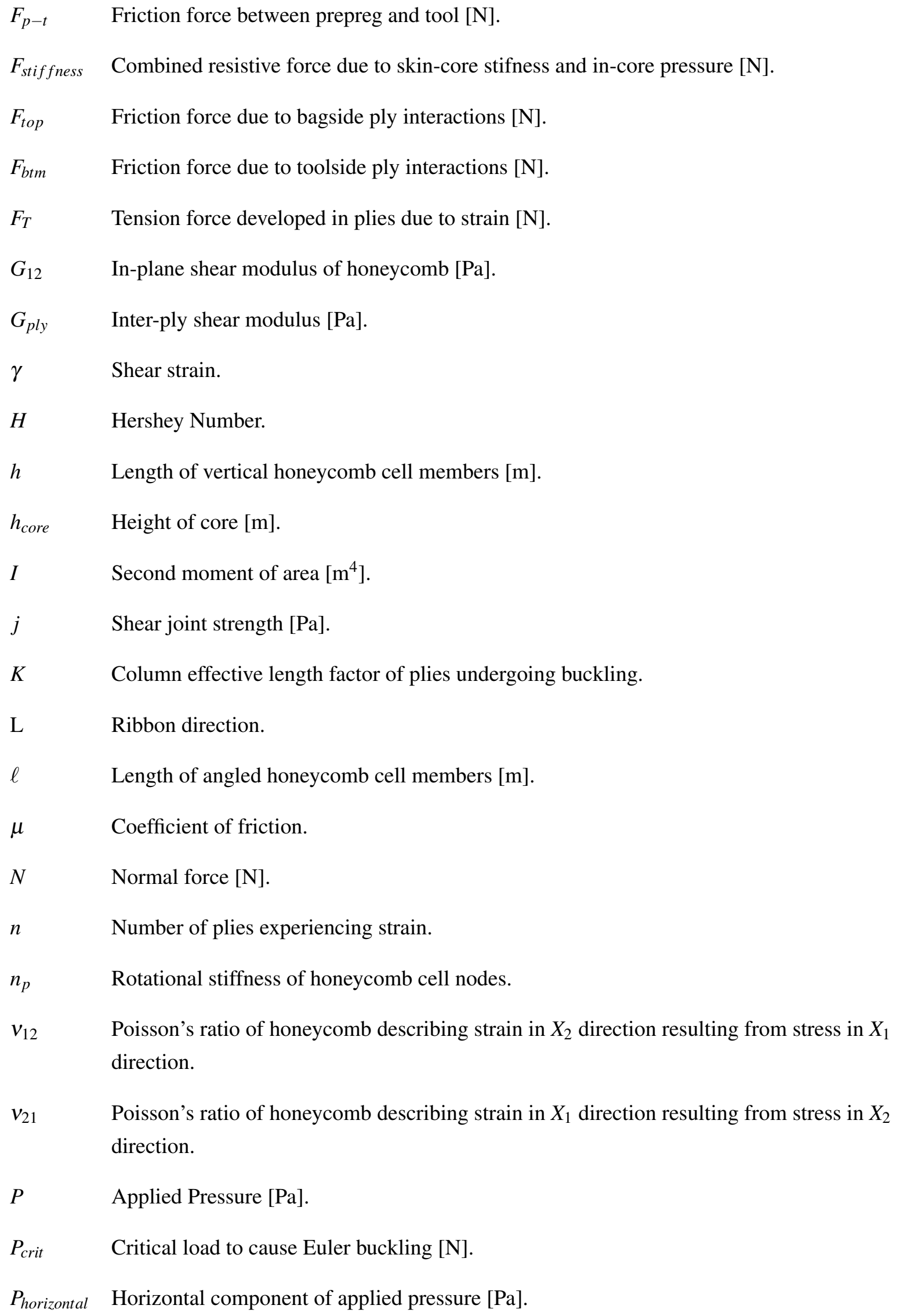




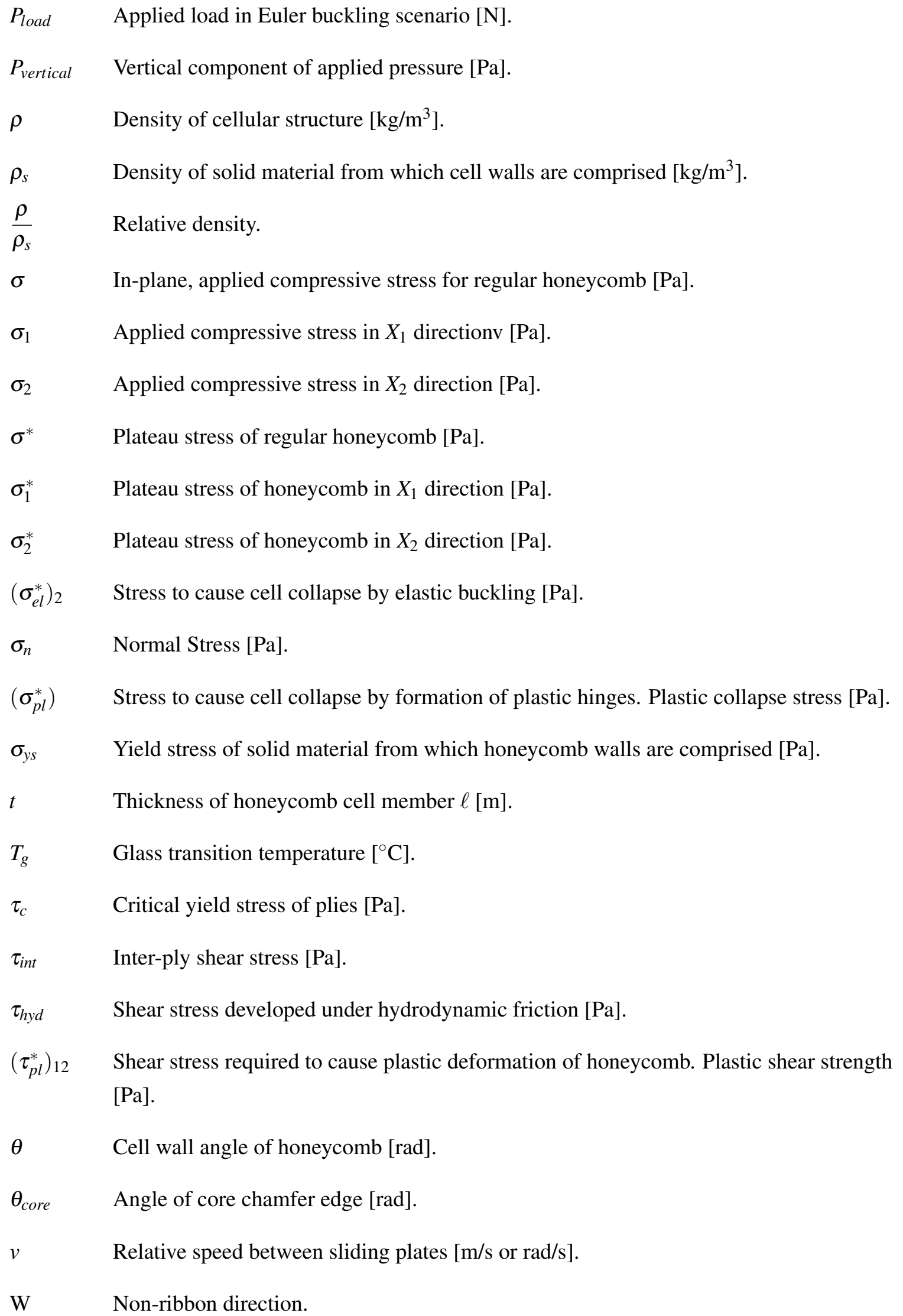


$w_{\text {core }} \quad$ Width of core $[\mathrm{m}]$.

$X_{1} \quad$ First axis of honeycomb core. Corresponds to the in-plane, non-ribbon direction.

$X_{2} \quad$ Second axis of honeycomb core. Corresponds to the in-plane, ribbon direction.

$X_{3} \quad$ Third axis of honeycomb core. Corresponds to the through-thickness or transverse direction. 


\section{List of Abbreviations}

AC Alternating Current

AISI American Iron and Steel Institute

BMS Boeing Material Specification

CFRP Carbon Fiber Reinforced Polymer

CMM Coordinate Measuring Machine

CMS Core Machining Stabilization

DOC Degree of Cure

GFRP Glass Fiber Reinforced Polymer

LCM Liquid Composite Molding

LVDT Linear Variable Displacement Transducer

MRCC Manufacturer Recommended Cure Cycle

NT Never Twisted

OX Overexpanded

PTFE Polytetrafluoroethylene

ST Standard Twist

UT Un-Twisted

VBO Vacuum-Bag Only 


\section{Glossary}

\author{
Bagside \\ Bending \\ Boundary lubrication \\ Chamfer edge \\ Chamfer radius \\ Core edge \\ Core machining stabilization
}

Core movement

Collapse (honeycomb mechanics) Failure of the honeycomb cells. Stress plateaus while strain

\section{Collapse (in-situ data)}

Coloumb friction

Critical Zone

Crush front increases significantly. Proceeds bending and precedes densification.

Top side of the core. Nearest the bag.

Linear, elastic bending of honeycomb cell walls. Precedes collapse.

Combination of coloumb friction and hydrodynamic friction arising from contact of two surfaces seperated by a viscous layer of molecular thickness.

Inclined edges of the core.

Top of the chamfer edge.

outside edge of the core.

Method to stabilize core for machining purposes and to mitigate core movement. Film adhesive precured to toolside of core.

Process induced deformation of sandwich panels resulting from compression of the core material due to external pressure.

Point where deformation of core is obvious, and beyond which, continuous, and significant. Corresponds to collapse of honeycomb cells.

Classical friction arising due to contact between two surfaces. Refer to Equation 2.16

Range of pressure between onset and collapse from the in-situ data. Represents the range of pressure over which core movement initiates.

The edge of the crush zone that is propagating across the panel. 
Crush zone

\section{Densification}

Dry core

Edgeband

Hydrodynamic friction

Laminate edge

Neat resin

NOMEX $^{\circledR}$

Non-ribbon direction

Onset

Overexpanded honeycomb

Panel edge

Pillowing

Prepreg

Regular honeycomb

Relative density

Ribbon direction

Sandwich panel
Region of the core that experiences densification during core movement.

Cell walls begin to touch each other. Proceeds collapse.

Honeycomb core without plies.

Region of panel laminate where all plies are located.

Friction arising from the presence of a viscous film layer of considerable thickness. Refer to Equation 2.15.

Region of the panel where no core is present. Between the panel edge and core edge.

Resin in absence of fibers.

A popular honeycomb material for composite sandwich panels.

Comprised of phenolic resin cured with aramid fibers.

Direction of honeycomb expansion. Perpendicular in-plane to the ribbon direction. Refer to Figure 2.1.

First point of observable movement in sandwich panel. LVDT sensors deviate from breather deformation pattern. Corresponds to bending of honeycomb cells.

Honeycomb that has been expanded past the regular configuration. The cell wall angle is less than $30^{\circ}$.

Outside edge of the panel.

Intrusion of plies into the core creating wrinkles.

Fibers/fabric pre-impregnated with resin.

Honeycomb with cell wall angles of $30^{\circ}$.

Ratio of cell density to that of the solid material from which the cell is comprised. Refer to Equation 2.1.

Direction of the glue lines from which honeycomb is expanded. Refer to Figure 2.1.

A structure comprising a low density core with stiff over and underlying facesheets for the purpose of increasing bending rigidity. 
Tie-down plies

Toolside
Plies that are restrained from moving.

Bottom side of the core. Nearest the tool. 


\section{Acknowledgments}

I would first and foremost like to thank my supervisor Professor Göran Fernlund. His knowledge and guidance made this thesis a possibility and has helped shape my professional outlook moving forward. I enjoyed our many discussions both in relation to this research and outside of the university.

I would also like to extend a special thanks to Professor Anoush Poursartip for filling in as supervisor while Göran was away. His insight and wisdom helped bring focus to this research. An additional thanks to Professor Reza Vaziri for the thoughtful discussions.

Thank you to The Boeing Company and especially Mark Shead who has been involved in the project since the beginning. Without his insight and experience, this project would not have been made a reality.

Thank you also to the folks of Convergent Manufacturing for providing me with the tools necessary to complete my research. In particular, I would like to thank Malcolm Lane and Trevor Fisher for their training and technical expertise.

I am grateful for all the help from each member of the Composite Research Network, particularly the professional staff. Dr. Casey Keulen for his consistent help on many aspects of the project and for training me on a variety of equipment, Dr. Navid Zobeiry for providing guidance and acting as an interim co-supervisor while Göran was away, Dr. Christophe Mobuchon for training me on the autoclave and the many enjoyable discussions we had, and Roger Bennett for assisting in technical matters. Thank you also to Ross Mcleod for helping with tooling for my project. I would like to extend a special thanks to Suzana Topic for her continual support and advice throughout my degree. I will miss Tuesday coffee breaks.

To my current and past friends and colleagues at the Composite Research Network, Hubert CourteauGodmaire, Caitlin Duffner, Scott Nesbitt, Cheng Chen, Gavin Tao, Shayan Fahimi, Shaghayegh Kiafer, Sahar Abouali, Mohammad Mohseni, Nasser Arbabi, Shayan Fahimi, Nima Bakhshi, Erfan Forghani, Margarita Galper, Dr. Andrew Stewart, Dr. Janna Fabris, Dr. Kamyar Gordnian, and John Park, thank you and welcome. Your friendship and fruitful discussions have made my time at UBC an enjoyable one. I would especially like to give a shout out to those students who began their degrees with me in September 2016, Hubert Courteau-Godmaire, Caitlin Duffner, and Cheng Chen. I would also like to acknowledge Dr. Johannes Reiner and Professor Martine Dube as well as visiting students Johannes Wiedemann, Alexandre Looten, Manish Nagaraj, Yuta Kumagai, Vincent Gill, Oliver Herb, and David Zimmerman. It was a pleasure to get to know you all and I hope to continue our friendships. To all current CRN students, I wish you all the best in the future.

Thank you to my funding sources, NSERC-CGSM. The funding received helped greatly offset the 
cost of living in Vancouver while completing a Master's Degree.

To all my friends I made while at UBC, thank you for the good times. From spending many days together in the mountains to pub trivia and heated board games matches, I enjoyed my time here and hope to continue seeing all of you around. I would especially like to thank Hubert Courteau-Godmaire, Stephen Ji, and Emma Kazic for being unreal roommates and all the huge plays.

I would also like to thank my parents, Mark and Michelle Pawson for their unwavering support and inspiring me to follow my passions. To my siblings, Kail, Danica, and Kenny Pawson, suhhh duh.

Finally, I would like to thank my partner Valentina Fazio for challenging me and helping keep things in perspective. I am beyond grateful for your continuing devotion and support. 


\section{Chapter 1}

\section{Introduction}

\subsection{Composites and sandwich panels}

A composite material is a multi-constituent material, wherein each component has differing physical and chemical characteristics. Typically a composite is comprised of a stiff, load-bearing component (reinforcement) coupled with a surrounding matrix material for force distribution. In advanced composite materials such as Glass and Carbon Fiber Reinforced Polymers (GFRP and CFRP respectively), the fibers act as the load-bearing component and the polymer as the matrix. A major benefit of composites and particularly CFRPs is that they offer exceptional specific strength characteristics. That is, they are strong and light-weight. This is advantageous in design, particularly in aerospace applications. Furthermore, the anisotropic nature of composites allows for tailoring of mechanical design to account for specific load paths. This lends itself to the importance of fiber architecture in composites for structural performance. There are a wide array of possible fiber orientations, including unidirectional or woven fabrics, which must be carefully chosen to suit the intended application.

Advanced composites are manufactured in several ways. Such methods include Liquid Composite Molding (LCM) processes whereby fibers are pre-placed in a mold and resin is injected into the mold via a pressure gradient and allowed to cure. Another common method is the use of fibers pre-impregnated with resin, known as prepreg, developed through a hot melt or solvent dip process. Prepreg manufacturing involves placing each individual layer on a tool which is then typically placed in an oven or autoclave to cure. This layer-by-layer construction is known as "laying up". Due to the anisotropy of composites, the pattern of layup greatly affects the mechanical performance of the final part and must be considered.

Polymer composites can be broken into two major categories, thermosets and thermoplastics, with the former being more common for prepreg applications. Thermosets begin in a viscous liquid state and undergo an irreversible transition through to a solid upon curing. Curing is achieved by subjecting the part to heat in order to allow for chemical rearrangement of the polymer chains into a cross-linked structure. Typically, the curing process is performed in an autoclave with the part placed under a vacuum bag to allow for external pressurization. This is done to achieve necessary consolidation of the part and prevent defects such as porosity and poor resin distribution. 
Two common types of composite structures that are used in design are laminates and sandwich panels. The former refers to a simple stack up of the composite in question (i.e. CFRP or GFRP) whereas the latter adds in an additional component, a core material. Sandwich panels typically consist of two constituent materials, a low-density core sandwiched between stiff under and overlaying facesheets or skins. This construction allows for increased bending stiffness without compromising on weight. The principle is similar to an I-beam, wherein the core represents the web and the facesheets represent the flanges. The additional thickness offered by the core increases the moment of inertia, with the locations of maximum bending moment (i.e the top and bottom surface) reinforced by the stiff facesheets. In composite panels, the facesheets will typically be a GFRP or CFRP material. Metal face-sheets are common outside of composites [5]. The core is generally a foam or honeycomb design, again with a wide range of materials used for each. The World War II military aircraft, the de Havilland Mosquito is often credited with being the first major structure to incorporate the use of sandwich panels [4, 5]. Both its wings and fuselage were made of a sandwich construction using plywood skins and a balsa wood core. Nowadays honeycomb core is becoming increasingly popular in sandwich panels, with both aluminum and NOMEX ${ }^{\circledR}$ used in engineering applications [4]. NOMEX ${ }^{\circledR}$ is the brand name for a textile made from aramid fibers, first developed by DuPont ${ }^{\mathrm{TM}}$ in the 1960s [18]. Aerospace grade NOMEX ${ }^{\circledR}$ honeycomb core is manufactured from sheets of NOMEX ${ }^{\circledR}$ paper adhesively bonded and then expanded to give the honeycomb pattern. The expanded structure is coated with phenolic resin and then cured to provide a stable shape.

\subsection{Core movement}

During processing of sandwich panels, the high pressure of the autoclave may actually result in deformation of the core in its weak, lateral direction. This problem is well known in the field of composites and is often referred to as core crush. However, core movement is becoming a preferred term in industry so to prevent confusion with core crush in the through-thickness direction. The term core movement is used in this body of work, although descriptive words such as "crush" may be used to illustrate the deformation process.

Core movement is often extreme and visible by the naked eye as seen in Figure 1.1. Deformed panels are irreparable and must be scrapped, making it one of the costliest manufacturing problems in composites fabrication [28]. The problem is so pervasive that processing methods are often altered to prevent core movement. This may include processing the parts at lower pressures than a traditional laminate. Moreover, specific tools and layup methods are used to mitigate the risk. Tie-downs are a common procedure wherein specific plies are restrained from moving, so to prevent deformation of the core itself (Figure 1.2). The idea being that if the core and immediate plies are well bonded, then by restraining ply movement, core movement is also prevented. This method has proved successful and is used extensively by major aerospace companies such as The Boeing Company. The problem, however, is that implementing tie-downs increases tooling and labour cost as well as production time. Moreover, because the physics of core movement is not well understood, the use of tie-downs is empirically based and not necessarily optimized. 

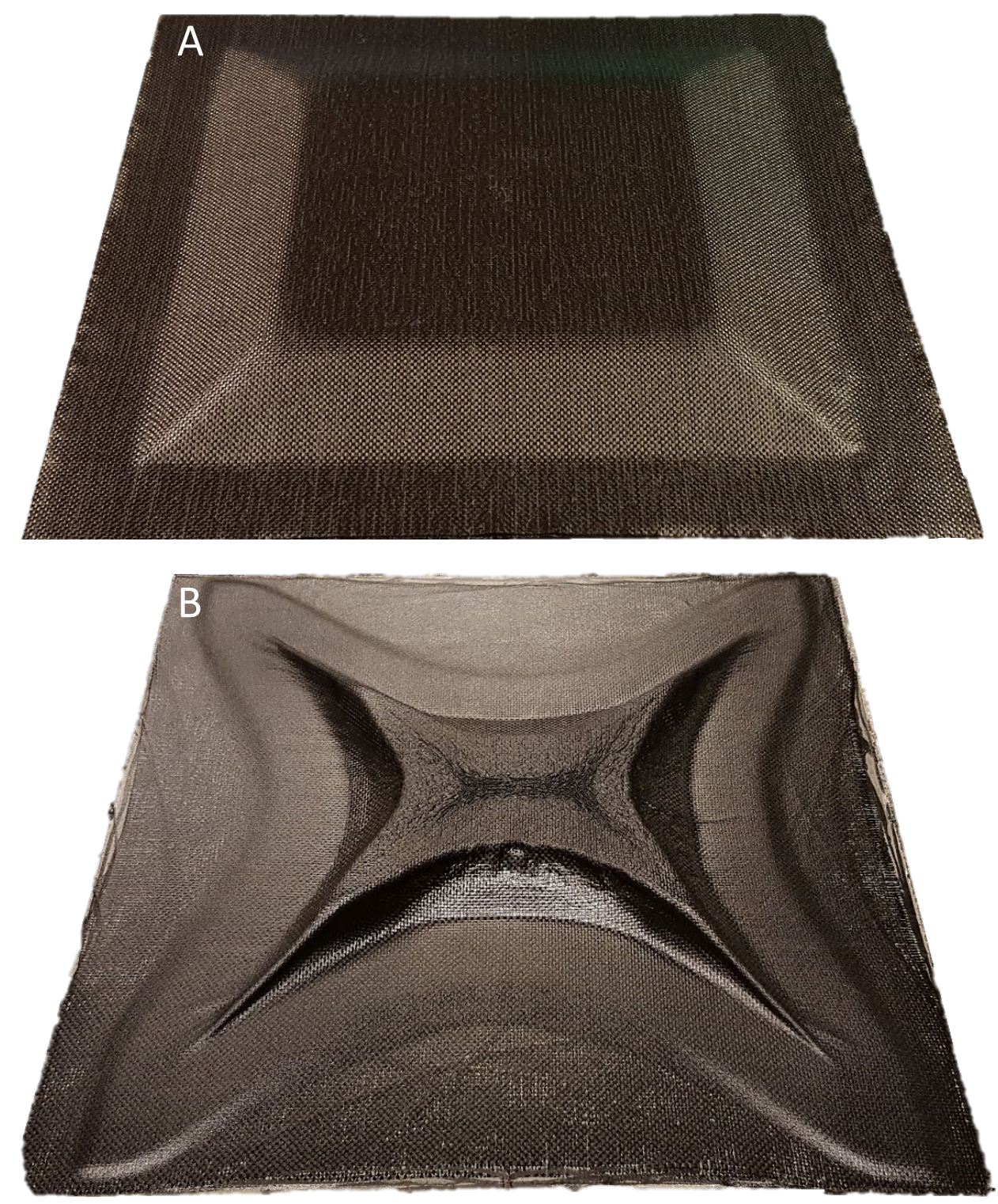

Figure 1.1: (A) Sandwich panel after layup and prior to cure. (B) Same panel following cure; drastic core movement has occured.

Much of the research on core movement focuses on the material aspect of the phenomena. That is, looking at the frictional resistance of various prepreg systems. However, there is a lack of fundamental knowledge regarding the mechanisms involved in the core movement process. Moreover, the processing conditions under which the phenomena occurs are not well defined. As such, methods to mitigate core movement are often based on empirical notions. There have been attempts to develop basic mechanical models for core movement [8, 28], however, these have yet to be validated. As of now, whether or not core movement will initiate during a given processing window remains largely unknown. This calls for a science based approach to understanding the driving factors involved in core movement during the processing of sandwich panels. By understanding the physics of the phenomenon, process maps can be 


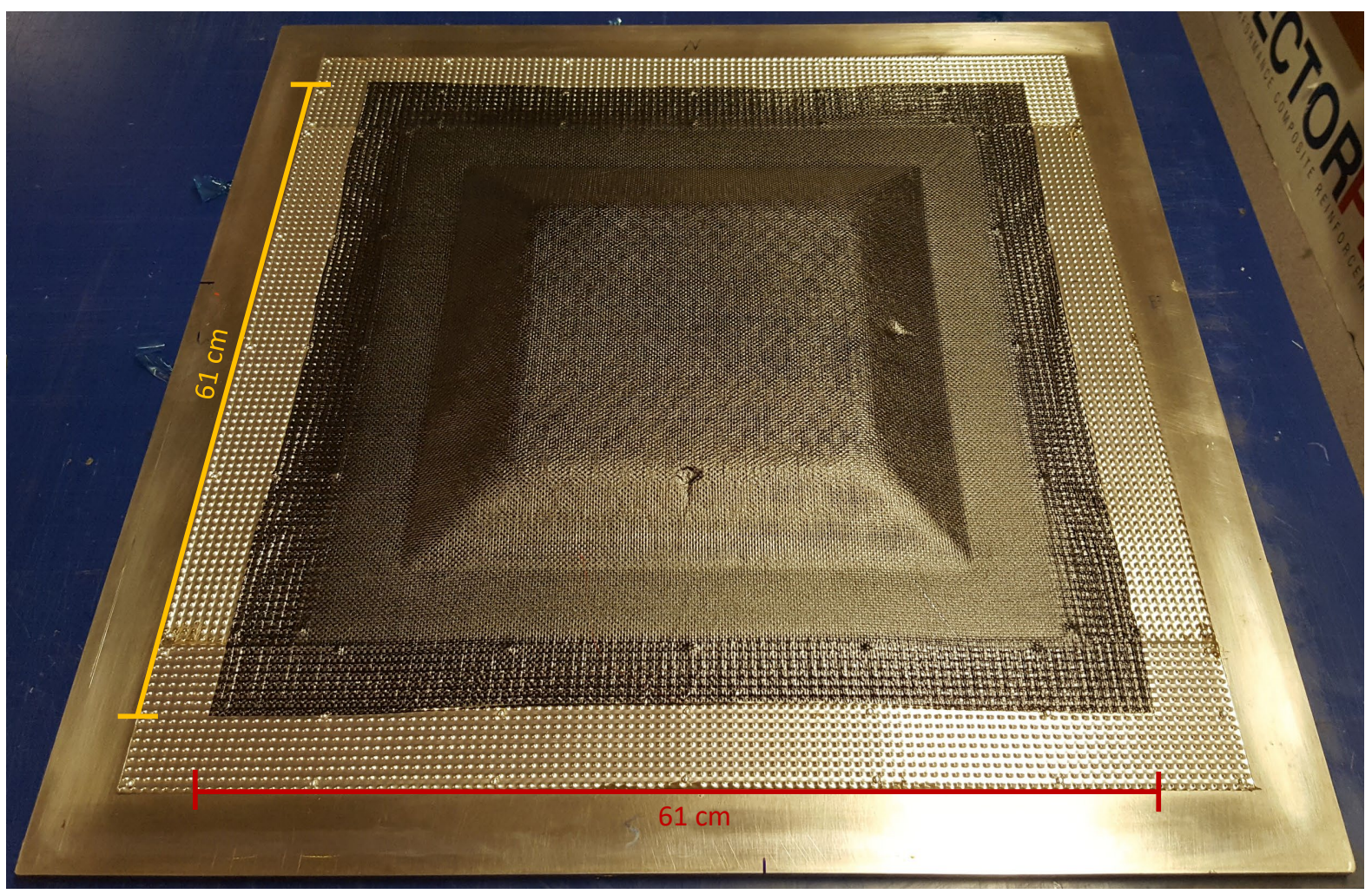

Figure 1.2: Processed sandwich panel on tool. Grit strips surrounding the part are used to restrain plies (tie-downs). Indents in the panel are from pressure sensors.

created to allow for prediction of core movement. This will provide manufacturers with the knowledge necessary to design for core movement. That is, mitigation methods can be tailored towards the panels and their processing window rather than employ a one-size-fits-all solution. 


\section{Chapter 2}

\section{Literature Review}

\subsection{Honeycomb mechanics}

Hexagonal honeycombs are the most common form used. The structural material can be a metal, polymer, or even ceramic. Polymer honeycombs, particularly NOMEX ${ }^{\circledR}$ honeycomb, is of importance in the context of this body of work and will be the focus of this section. Furthermore, as it pertains to core movement, in-plane deformation due to compression will be discussed as opposed to out-of-plane deformation and deformation due to tension.

\subsubsection{Terminology}

Many honeycombs are manufactured by expanding sheets of glued paper strips or ribbons. This gives directionality to the honeycomb structure as seen below in Figure 2.1. The in-plane dimensions of a honeycomb structure refer to the $X_{1}-X_{2}$ plane. The out-of-plane or through-thickness dimension is the $X_{3}$ direction as shown in the figure [22,49]. The $X_{2}$ direction is known as the ribbon (L) direction since it follows the direction of the glued ribbons. Correspondingly, the $X_{1}$ direction is known as the non-ribbon (W) direction. For the sake of consistency the terms $\mathrm{L}$ and $\mathrm{W}$ will be used to refer to the $X_{2}$ and $X_{1}$ direction respectively. Regular honeyomb are expanded such that the unit honeycomb cell has equal side lengths with the angle between each side being $120^{\circ}$. Overexpanded (OX) honeycomb is also available wherein the cellular structure is no longer regular, so to provide increased mechanical benefits in the $\mathrm{W}$ direction and improve formability [10]. Finally, honeycomb may also be manufactured by bonding corrugated sheets as opposed to expanding glued sheets. This is common in aluminum honeycomb. A hexagonal honeycomb unit cell is shown in Figure 2.2.

Hexcel is a common supplier of honeycomb materials. Their aramid lineup is given the following designation [25]:

$$
\text { Material - Cell Size - Density }
$$

Where, material refers to the honeycomb type, cell size refers to the size of the honeycomb unit cell (as shown in Figure 2.2), and density refers to the nominal density of the honeycomb material in pounds per 


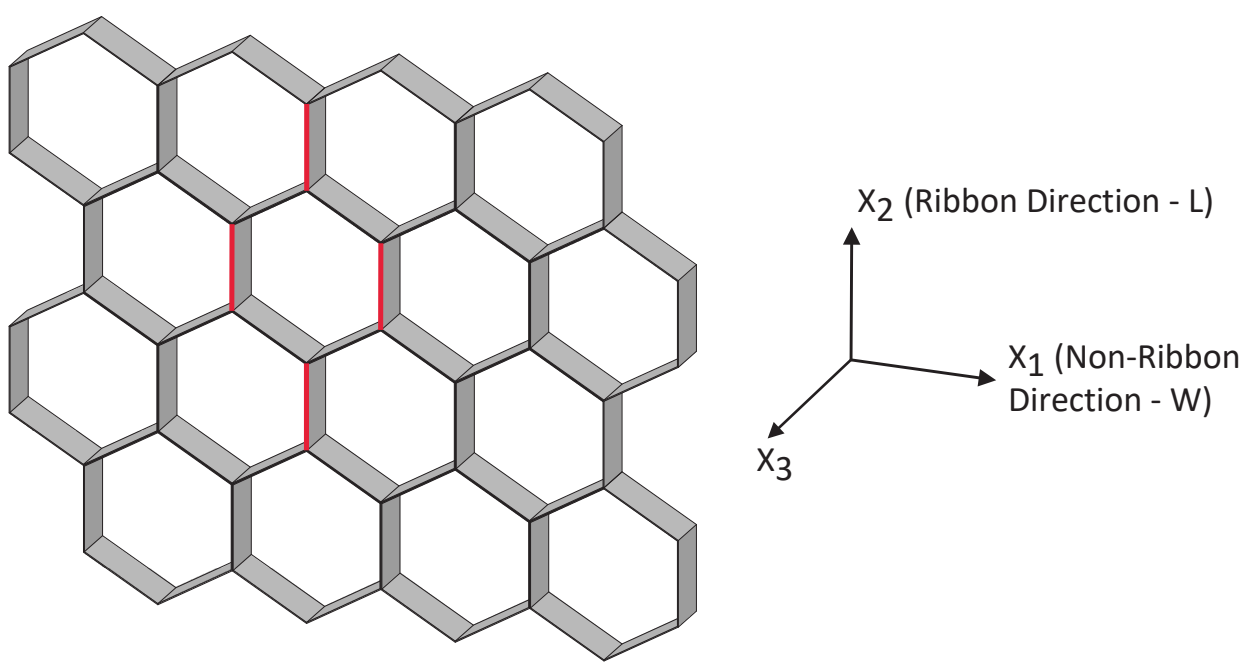

Figure 2.1: Hexagonal honeycomb structure. Glue lines are present in the ribbon direction (L) as indicated by the red lines. The non-ribbon direction $(\mathrm{W})$ is perpendicular in-plane to this.

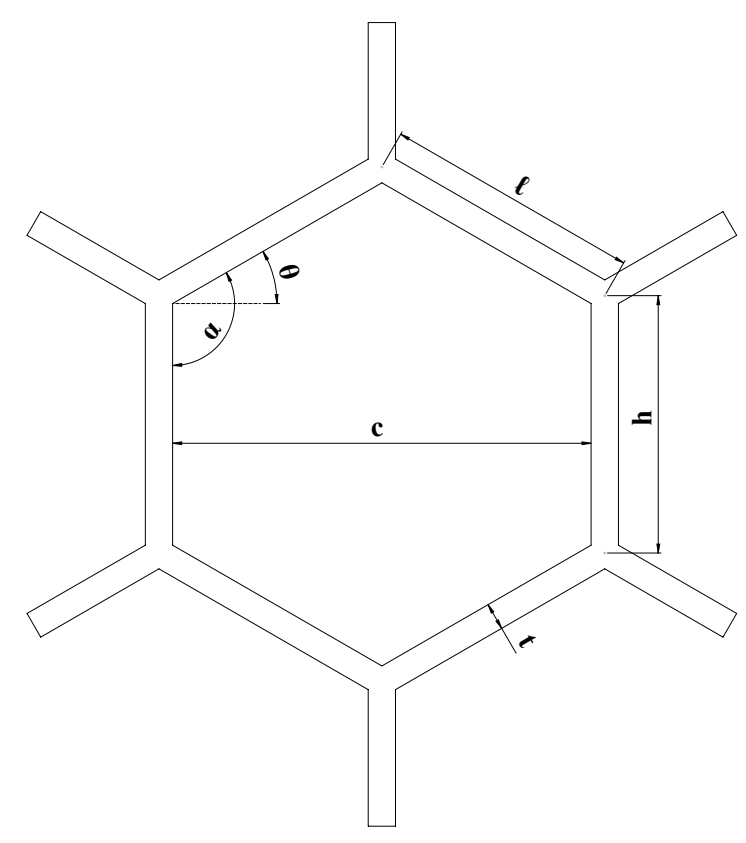

Figure 2.2: Honeycomb unit cell, where $\ell$ represents length, $h$ height, $t$ thickness, $c$ cell size, $\alpha$ the angle between each side, and $\theta$ the cell wall angle. For regular honeycomb structures $h=\ell$, $\alpha=120^{\circ}$, and $\theta=30^{\circ}$. The out-of-plane thickness is denoted as $b$. 
cubic foot. An example of a NOMEX ${ }^{\circledR}$-based honeycomb product designation from Hexcel is shown below.

$$
\text { E.g.: HRH-10 - 1/8 - } 3.0
$$

\subsubsection{Relative density}

Relative density is a highly important factor in determining the properties of a foam or honeycomb material [7, 22, 49]. It is defined as the ratio of cell density to that of the solid material, or simply:

$$
\text { Relative Density }=\frac{\rho}{\rho_{s}}
$$

Where, $\rho$ is the density of the cellular structure and $\rho_{s}$ is the density of the solid material from which the cell walls are comprised. Cell density is sometimes displayed with an asterisk [22].

Relative density is a function of the cell wall thickness $(t)$ and edge-lengths ( $\ell$ and $h)$. It can, in general, be written as follows:

$$
\frac{\rho}{\rho_{s}}=\frac{t / \ell(h / \ell+2)}{2 \cos \theta(h / \ell+\sin \theta)}
$$

For regular honeycomb where $h=\ell$ and $\theta=30^{\circ}$, then:

$$
\frac{\rho}{\rho_{s}}=\frac{2 t}{\sqrt{3} \ell}
$$

Therefore, it can be said that honeycombs with thicker cell walls have higher relative density, whereas honeycombs with longer edges, and therefore a larger cell size exhibit lower relative density, as would be expected.

It should be noted that the expansion method of honeycomb manufacturing results in a doubling of the cell wall thickness for members of length $h$ in Figure 2.2. This does not affect the in-plane Young's Moduli nor Poison's ratios, however it does affect the in-plane shear modulus and relative density. That said, if the ratio of $t / \ell$ is small (i.e. $t<<\ell$ ), meaning the relative density is low, then it can be assumed that thickness is constant amongst all cell walls [22]. This is assumed in the calculations that follow.

\subsubsection{Uniaxial loading}

The functional loading direction of honeycomb cores in sandwich panels is along their stiff, throughthickness direction (the $X_{3}$ direction as shown in Figure 2.1) [49]. However, it is the relatively large in-plane, compressive loads that lead to the deformation of the cellular structure that is core movement. In-plane compression of honeycomb results in three distinct regions. They are, in order of progression, bending, collapse, and densification [7, 11, 22, 38, 39]. Bending constitutes a linear elastic regime wherein there is an initial constant rise in the stress strain diagram. Cellular collapse may be the result of elastic buckling, formation of plastic hinges, or fracture of the cell walls. The manner in which collapse occurs is material dependent. It is characterized by a plateau region on the stress-strain curve, wherein deformation occurs under relatively constant stress due to failure of the cellular structure. Finally, 


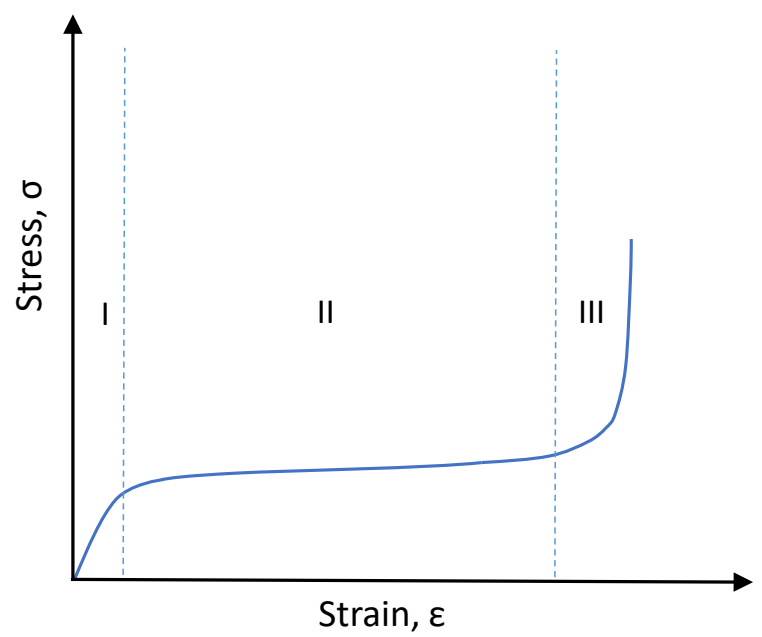

Figure 2.3: Typical compressive stress-strain curve for an elastomeric honeycomb. I: Bending linear elastic bending of cell walls, II: Collapse (plateau region) - constant stress as cells fail, III: Densification - cell walls touching. Figure adapted from [22].

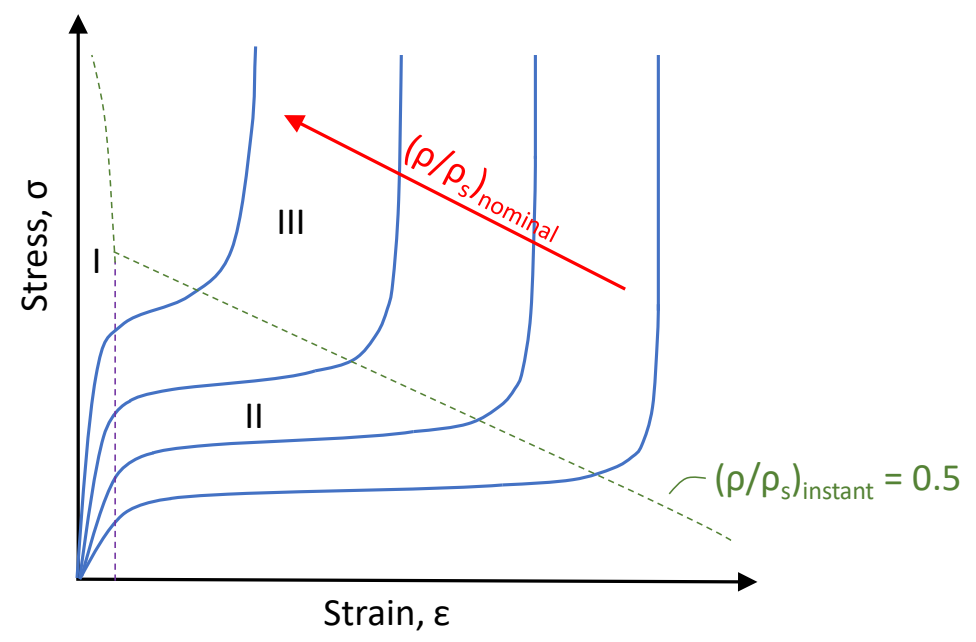

Figure 2.4: Effect of increasing nominal relative density on the compressive stress-strain response of honeycombs. I: Bending - linear elastic bending of cell walls, II: Collapse (plateau region) - constant stress as cells fail, III: Densification - cell walls touching. Figure adapted from [22].

densification occurs when opposing cell walls begin to touch. This is marked by an exponential rise in stress, as the cells close up and the structure increases in density. The magnitude of each regime is dependent on the relative density. Higher relative densities result in a stiffer initial response, a shorter (or less severe) plateau section, and earlier onset of densification. A typical stress-strain curve for an elastomeric honeycomb is shown in Figure 2.3. Similarly, Figure 2.4 shows how the stress strain response is affected by an increase in the nominal relative density. It should be noted that relative density increases as the cellular structure is compressed, due to closing of the cell walls. In fact, the onset of densification is defined as the point when the instantaneous relative density becomes 0.5 [22]. 
NOMEX $^{\circledR}$ honeycomb has been shown to exhibit a similar stress-strain curve as that shown in Figure 2.3 under compression in both the ribbon and non-ribbon direction [23]. That said, the manner in which collapse occurs is different for loads applied in the ribbon versus non-ribbon direction. Initially, in both cases the cell members marked $\ell$ in Figure 2.2 bend while the L direction members (marked $h$ in Figure 2.2) remain straight. Once the elastic limit is reached, loads applied in the L direction result in a homogeneous collapse of the cellular structure by folding of the $h$ members. In contrast, loads applied in the $\mathrm{W}$ direction result in a row-by-row cellular collapse pattern, wherein $h$ members are forced together. The first row(s) to collapse appear arbitrary [23]. In the case of aluminum honeycombs, local row-by-row collapse occurs in both the ribbon and non-ribbon direction [38, 39]. In general, for elastic honeycombs, such as NOMEX ${ }^{\circledR}$ or those with high hardening properties, homogeneous collapse dominates (at least in the ribbon direction). This gives a nonlinear, monotonically increasing stressstrain response [31, 38]. It should be noted that for $\mathrm{NOMEX}^{\circledR}$ the slope of the plateau region is still increasing slightly. This effect is more obvious in the L direction due to uniform cell collapse as opposed to localized failure. The general shape of the stress-strain response of NOMEX $^{\circledR}$ is independent of strain rate for both $\mathrm{L}$ and $\mathrm{W}$ loading, although higher strain rates result in an overall stiffer response, especially in the $\mathrm{L}$ direction due to the aforementioned uniform failure pattern [23].

\subsubsection{Linear elastic bending}

Similar to relative density, Young's modulus and shear modulus for honeycombs are given relative to the modulus of the solid material from which the cell walls are constructed. Due to their anisotropy, honeycombs have four independent moduli $\left(E_{1}, E_{2}, G_{12}, v_{12}\right)$ and two plateau stresses $\left(\sigma_{1}^{*}, \sigma_{2}^{*}\right)$, relating to loading in the $\mathrm{W}$ and $\mathrm{L}$ direction. However, for regular honeycombs $\sigma_{1}^{*}=\sigma_{2}^{*}=\sigma^{*}$ and $E_{1}=E_{2}=E$, resulting in only three independent moduli and one plateau stress [22]. For the purpose of being concise and as it pertains to this body of work, only the equations for regular honeycombs will be discussed. The equations describing linear elastic bending of regular honeycombs and their importance are shown below [22]:

Young's Modulus:

$$
\frac{E}{E_{s}}=2.3\left(\frac{t}{\ell}\right)^{3}
$$

Since the Young's modulus is only a function of $t / \ell$, just as relative density is, it can be said that a one-fold increase in relative density results in a cubic increase in compressive stiffness.

Poisson's Ratio:

$$
v_{12}=v_{21}=1
$$

Equation 2.5 has important implications as it means that in-plane compression of honeycomb in one direction results in an equivalent magnitude of expansion in the other in-plane direction. The through- 
thickness $\left(X_{3}\right)$ direction does not experience any deformation, however. In a solid material, a Poisson's Ratio of 1 is impossible as the material behaves in a three-dimensional manner; deformation along any axis results in deformation along the other two axes. However, honeycomb behaves as a hinged, geometrical material, allowing it to act as a two dimensional structure in-plane.

Shear Modulus:

$$
\frac{G_{12}}{E_{s}}=0.57\left(\frac{t}{\ell}\right)^{3} \approx \frac{E}{4 E_{s}}
$$

Similar to compressive stiffness, it can be said that a one-fold increase in relative density results in a cubic increase in shear stiffness.

It should be noted that when polymeric honeycombs approach their glass transition temperature, $T_{g}$, the cell walls no longer bend in a linear-elastic fashion but rather undergo viscoelastic deformation [22]. In the case of $\mathrm{NOMEX}^{\circledR}$ honeycomb the glass transition temperature occurs around $300^{\circ} \mathrm{C}$ [9]. Since this body of work only deals with $\mathrm{NOMEX}^{\circledR}$ honeycomb, at a maximum processing temperature of $180^{\circ} \mathrm{C}$, viscoelastic deformation of honeycomb is not discussed.

\subsubsection{Cell collapse}

Following bending, as mentioned above, there are three modes in which the cell structure may fail. That is, elastic buckling, plastic collapse, and brittle failure [22]. Elastic buckling and plastic collapse are shown below as they relate to polymers. Note that an asterisk denotes properties pertaining to that of the plateau region.

Elastic buckling only occurs in the ribbon direction, since members $h$ are aligned with the applied load. That is to say that at a critical load (i.e. $P_{\text {load }}=P_{\text {crit }}$ ), the members parallel to the loading direction will buckle like an Euler column [11, 22]. This critical load is displayed as follows:

$$
P_{c r i t}=\frac{n_{p}^{2} \pi^{2} E_{s} I}{h^{2}}
$$

Where, $I=b t^{3} / 12$ for a simple beam and $n_{p}$ refers to the rotational stiffness of the cell nodes where the walls meet. $P_{\text {load }}=2 \sigma_{2} \ell b \cos \theta$ (where $\sigma_{2}$ is the applied stress) and for regular hexagonal honeycombs, $n_{p}=0.69$ [22]. Therefore:

$$
\frac{\left(\sigma_{e l}^{*}\right)_{2}}{E_{S}}=0.22\left(\frac{t}{\ell}\right)^{3}
$$

Combining Equation 2.8 and Equation 2.4 yields:

$$
\left(\varepsilon_{e l}^{*}\right)_{2}=\frac{1}{10}
$$

Note that the subscript "2" in Equation 2.8 and Equation 2.9 denotes the loading direction, as buckling only occurs in the $\mathrm{L}$ (i.e. $X_{2}$ ) direction.

In the case of plastic collapse, plastic hinges form at the nodes, meaning further deformation of 
the cell structure is unrecoverable. This occurs when the the bending moment reaches the fully plastic moment, giving a plastic collapse stress of $\sigma_{p l}^{*}[22,31]$.

Once again, in the case of regular honeycombs, the plastic collapse stress is the same for both the $\mathrm{L}$ and $\mathrm{W}$ direction and is a function of the solid material yield stress. It is denoted as [22]:

$$
\frac{\sigma_{p l}^{*}}{\sigma_{y s}}=\frac{2}{3}\left(\frac{t}{\ell}\right)^{2}
$$

Similarly, a shear load can be applied beyond the plastic limit, such that honeycomb will undergo plastic shear deformation. For regular honeycombs the plastic shear strength is denoted as [22]:

$$
\frac{\left(\tau_{p l}^{*}\right)_{12}}{\sigma_{y s}}=\frac{1}{2 \sqrt{3}}\left(\frac{t}{\ell}\right)^{2}
$$

It is possible for both elastic buckling and plastic collapse to occur with the former proceeding the latter. It has been shown that this occurs at a critical value of $t / \ell$ (thereby relating to the relative density of the material). For regular honeycombs this value is defined as follows [22]:

$$
\left(\frac{t}{\ell}\right)_{c r i t}=3 \frac{\sigma_{y s}}{E_{s}}
$$

\subsubsection{Biaxial loading}

Linear elasticity under biaxial loading follows much of the same response as that for uniaxial loading. Collapse however changes, as the two stresses now influence one another and the buckling mode changes. This complicates the calculations of elastic buckling and is therefore not shown. The pattern of deformation seems to be a combination of that seen in uniaxial loading of the $\mathrm{L}$ and $\mathrm{W}$ direction [22].

$E$ and $v_{12}$ hold the same relation as those laid out in 2.4 and 2.5. However, axial deformation of the cell walls must now be considered. It was previously ignored, as bending was the predominant mode of deformation. Taking this into account and assuming that the two applied stresses are equal $\left(\sigma_{1}=\sigma_{2}=\sigma\right)$, then the strain response in the $\mathrm{L}$ and $\mathrm{W}$ direction are equivalent and can be written as follows [22]:

$$
\varepsilon=\frac{\sqrt{3} \sigma}{E_{S}(t / \ell)}
$$

In the case of plastic collapse for a regular honeycomb where the applied stresses are equal, the following relation can be obtained [22, 31]:

$$
\frac{\sigma_{p l}^{*}}{\sigma}=\frac{4}{3}\left(\frac{t}{\ell}\right)^{2}
$$




\subsection{Inter-ply friction of prepreg}

Inter-ply friction of carbon/epoxy prepreg systems refers to the friction between plies in a stack up. Not to be confused with intra-ply friction, which refers to friction between tows within a single ply. When the inter-ply shear force exceeds that of the inter-ply friction, relative movement of plies will occur. Generally speaking, there are two main modes of friction between articulating surfaces. That is, hydrodynamic friction and coulomb (or dry) friction. Hydrodynamic friction is present when a viscous film of appreciable thickness exists between two surfaces. It is a function of film thickness, viscosity, and sliding rate according to Equation 2.15. Coulomb friction is the classical friction description between two surfaces and exists when no fluid interface is present. It is governed by the normal force and coefficient of friction according to Equation 2.16.

$$
\begin{gathered}
\text { Hydrodynamic Friction: } \\
\tau_{\text {hyd }}=\frac{\eta}{d} v
\end{gathered}
$$

Where, $\tau_{\text {hyd }}=$ hydrodynamic shear stress, $\eta=$ viscosity, $d=$ film thickness, and $v=$ relative speed between plates.

\section{Coloumb Friction :}

$$
F_{f}=\mu N
$$

Where $F_{f}=$ coulomb friction force, $\mu=$ coefficient of friction, and $N=$ normal force.

Of course, it is possible for both modes to be present. If the film separating two surfaces is of molecular thickness, it is considered to be under boundary lubrication and follows a behaviour similar to that of coloumb friction [34]. If both boundary (or pure coloumb friction) and hydrodynamic lubrication are present, it is referred to as mixed lubrication [34, 45]. In the case of prepreg there will be points where the two surfaces are in direct contact, separated only by a thin film, and areas where they are separated by a film of considerable thickness, resulting in mixed lubrication. The Stribeck Curve provides a visual for lubrication friction regimes, wherein the coefficient of friction is mapped against the Hershey Number, as shown in Figure 2.5 [34, 44]. The curve allows for the governing friction mechanisms at different processing conditions to be determined, wherein processing conditions are represented by the Hershey Number. That is to say, depending on the friction coefficient at a given Hershey Number one can tell whether they are in a state of boundary, mixed, or hydrodynamic lubrication. The Hershey Number is a function of the viscosity, sliding rate (as presented above), and applied pressure (P), according to Equation 2.17. Note that $v$ typically denotes rotational motion when referring to the Hershey Number. Hence, the Hershey Number is often presented as dimensionless.

$$
H=\frac{\eta v}{P}
$$

Inter-ply friction between prepreg layers changes throughout the cure cycle. Initially, when there is a considerable film thickness, hydrodynamic friction dominates. As temperature is ramped up, viscosity 


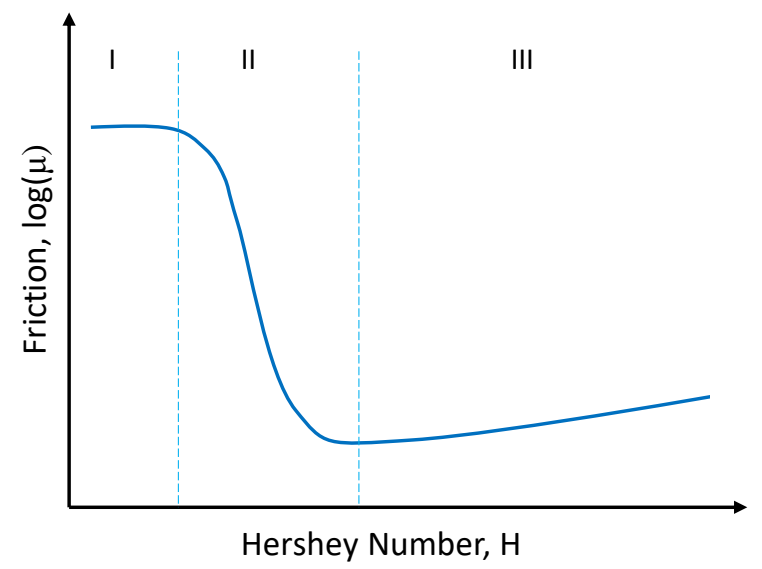

Figure 2.5: Stribeck Curve demonstrating three regions of lubrication. I: Boundary lubrication, II: Mixed lubrication, III: Hydrodynamic lubrication. Figure adapted from [34].

decreases and inter-ply friction thus also decreases. However when the viscosity is low enough, this effect is no longer seen and, in fact, inter-ply friction is shown to increase [19, 20, 34, 35]. This increase in friction at low viscosity has been associated with an insufficient buildup of film thickness, allowing for mechanical interaction between fibers. In other words, the friction response transitions from a hydrodynamic regime to boundary lubrication, resulting in a coloumb dominated friction response at low viscosity. However, some material systems seem apathetic to changes in viscosity at viscosities above $250 \mathrm{~Pa} \cdot \mathrm{s}$, with a friction behaviour that remains coloumbic in nature [34].

It has also been shown that as normal force increases, friction initially decreases but eventually stabilizes in accordance with boundary lubrication [30,34]. This has been attributed to the reduction of surface roughness under pressure until the prepreg can no longer be consolidated further and roughness remains constant.

\subsubsection{Pre-yield behaviour}

When the shear stress acting on a laminate exceeds the inter-ply frictional resistance, yielding occurs. That is, slip or relative movement between plies occur. It is this inter-ply slip that allows for the progression of core movement. As such, pre-yield behaviour is of importance.

Erland et al [19] describe inter-ply shear using a viscoelasto-plastic model, wherein the viscoelastic contribution pertains to the pre-yield behaviour. The relation is given as:

$$
\tau_{i n t}=G_{p l y} \gamma+\eta \frac{d \gamma}{d t}
$$

Where $\tau_{i n t}=$ inter-ply shear stress, $\gamma=$ shear strain, $G_{p l y}=$ inter-ply shear modulus, and $\frac{d \gamma}{d t}=$ shear strain rate. Yield occurs when the inter-ply shear stress exceeds the critical yield stress (i.e. $\tau_{\text {int }} \geq \tau_{c}$ ). The critical yield stress is given as a function of the coloumb and hydrodynamic friction, according to Equation 2.19. 


$$
\tau_{c}=\mu \sigma_{n}+j
$$

Where $\sigma_{n}=$ normal stress and $j=$ shear joint strength. Shear joint strength is similar to tack and represents the hydrodynamic portion of the friction response. As mentioned earlier, inter-ply friction decreases upon heating to a minimum value after which it increases again. This is marked by a change in friction response from one dominated by hydrodynamic lubrication to one dominated by boundary lubrication. A similar response is shown when it comes to the critical yield stress [19]. That is, $\tau_{c}$ decreases upon heating, reaches a minimum value, and then increases again slightly. Slip is most dominant when the critical yield stress is at its minimum. The changes in $\tau_{c}$ with temperature can be attributed to the affects of $\mu$ and $j$. The latter, $j$, highest within the hydrodynamic regime, begins at a maxima and decreases with an increase in temperature. Conversely, $\mu$, highest during boundary lubrication, begins at a minima and increases with an increase in temperature [19]. These contradictory affects are related to the amount of surface resin present [19, 28]. Initially, the surface resin layer is thick and resistance to shear is dominated by properties of the resin matrix. As temperature is ramped up, the softened friction response is due to a decrease in the shear modulus of the resin. With continual heating, the resin migrates to the core of the plies [16, 43, 50], allowing for improved fiber intermingling and an increase in dry friction. Therefore, the shear response at low temperature processing is dominated by the resin, whereas at high temperature, the shear response is dominated by fiber-fiber contact. The magnitudes constituting "high" versus "low" temperature processing are dependent on the material system [34].

\subsection{Core Movement}

\subsubsection{Physics of core movement}

Core movement occurs when the lateral force acting on the panel exceeds the resistive forces of the panel. The lateral force manifests through the horizontal component of pressure acting along the chamfer edge of the panel. Brayden and Darrow [8] hypothesized that the resistive forces are a combination of the frictional resistance of the prepreg, the compressive strength of the core and plies, and the force exerted by entrapped gas filling the core volume. The latter variable was identified as the predominant factor resisting core collapse. This model, however, wrongly assumes frictional resistance to be negligible without evidence supporting the notion. Alteneder et al [6] expanded this work by claiming that it is the difference in autoclave pressure and internal core pressure that is the driving force for core movement. They correlated the extent of core deformation with the level of in-core pressure and showed that in-core pressure could be manipulated to delay or prevent the onset of core movement.

In a multivariate design of experiment, Renn et al [42] further showed that manipulation of the internal core pressure greatly influenced core movement. Vacuum pressure during layup was identified as the primary factor responsible for dictating in-core pressure during autoclave processing. However, the resin system was found to be the most important factor influencing core movement resistance; although, they were unsure how exactly how. 
Martin et al [35, 36] noted that in order for core movement to occur, plies must slip across an interface. They developed a method for measuring frictional resistance of woven prepregs and showed that inter-ply friction does in fact play a key role in dictating honeycomb core movement [35]. As such, the magnitude of friction is dictated by temperature according to the properties of inter-ply friction as described above. In the hydrodynamic regime, viscosity dictates inter-ply friction. However, if little surface resin is present (i.e the film layer is thin), fiber intermingling occurs and boundary lubrication dominates. In a first-of-its-kind study, Martin et al [35] showed that both viscosity and surface resin distribution affect frictional resistance, which ultimately influences core movement. Moreover, in a separate study [36], they hypothesized that the impregnation level of the prepreg should affect core movement by influencing the resin distribution. It was shown that, for unidirectional fibers, fiber tension was the only impregnation condition that had any significant affect on core movement. Prepregs with low-tensioned fibers displayed a higher concentration of fibers near the prepreg surface. This allowed for greater mechanical interaction with adjacent plies and thus increased the inter-ply friction and reduced the severity of core movement. Martin and Seferis [37] then further showed that resin systems could be tailored to improve their frictional characteristics by adjusting the concentration of elastomer content. Doing so, would effectively alter the amount of surface resin present during cure.

Pelton et al [40] developed a similar testing rig to quantify frictional values associated with core movement for specific GFRP and CFRP systems. They showed that for carbon fabrics, fiber type, in addition to the resin system, plays a large role in influencing friction and thus dictating core movement. Similarly, Hsiao et al [28] demonstrated that fiber architecture, plays a crucial role in determining frictional resistance and the extent of core movement.

Hsiao et al [28] built upon the initial free body diagram laid out by Brayden and Darrow [8] to include frictional resistance at the ply level, as seen in Figure 2.6. This includes friction between the bottom ply and the tool $\left(F_{p-t}\right)$, friction between plies $\left(F_{p-p}\right)$, and friction between the top ply and the bag $\left(F_{p-b}\right)$. In this model, the applied force is transferred through the plies by a series of shear interactions, wherein the plies nearest the core experience the greatest shear force. Assuming the core and prepreg are well bonded, then in order for the core to deform, slippage must occur along a bagside and toolside interface [28, 36]. Hsiao et al [28] postulate that slippage occurs across the interfaces of least friction, wherein the prepreg-bag interaction $\left(F_{p-b}\right)$ presents the highest friction and the prepreg-tool interaction $\left(F_{p-t}\right)$ presents the lowest friction. The following expression can be made [28]:

$$
F_{p-b}>F_{p-p}>F_{p-t}
$$

Therefore, bagside slippage is assumed to occur across a prepreg-prepreg interface $\left(F_{p-p}\right)$, whereas toolside slippage occurs across the prepreg-tool interface.

Hence, the condition for core movement becomes [28]:

$$
F_{p-p}+F_{p-t}+F_{\text {stiffness }}<P_{\text {horizontal }} \cdot A_{\text {core }}
$$

Or more specifically: 


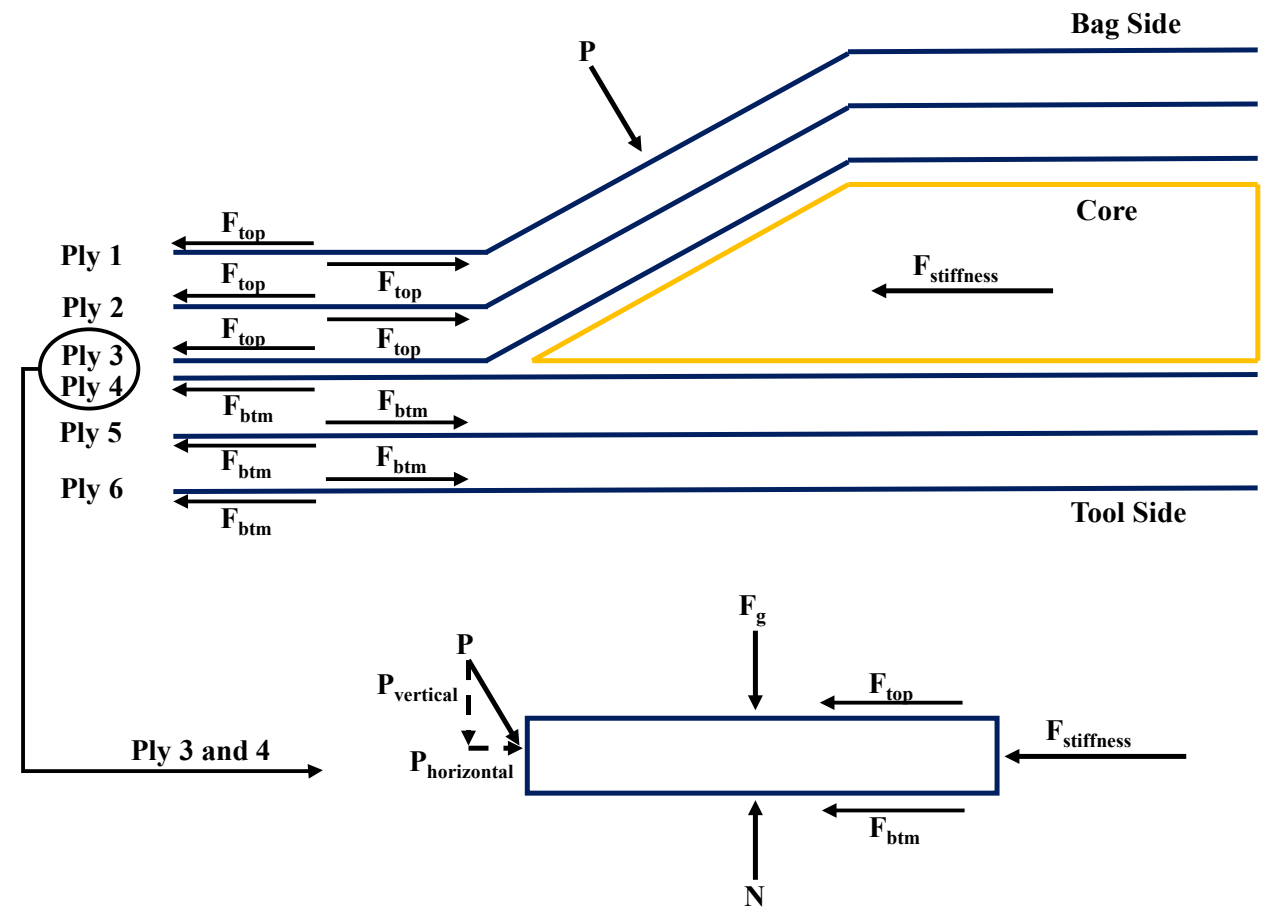

Figure 2.6: FBD of sandwich panel demonstrating forces involved in core movement. $F_{\text {stiffness }}$ includes both the material stiffness of the skins and core in the lateral direction as well as the in-core pressure. Figure adapted from [28].

$$
F_{p-p}+F_{p-t}+F_{\text {stiffness }}<P \sin \theta_{\text {core }} \cdot h_{\text {core }}
$$

Where $F_{\text {stiffness }}$ encompasses the material stiffness of the core and uncured plies, as well as the the in-core pressure. $\theta_{\text {core }}$ represents the chamfer angle of the core and $h_{\text {core }}$ is the height of the core with unit width into-the-page.

This model represents the most up-to-date core movement model. That said, it isn't without limitations. The manner in which ply slippage occurs according to Equation 2.20 is speculative. It also assumes this condition holds true for all material systems, which may not be the case considering their frictional values can be quite different [34, 35]. Furthermore, forces arising from in-core pressure ply stiffness, and interface friction evolve during cure and are therefore transient. This makes the evaluation of such a condition as presented in Equation 2.22 quite challenging. It also adds a further condition that if the frictional forces are transient then the assumption laid out in Equation 2.20 must hold true throughout the cure cycle for the model to be valid. These limitations have not yet been discussed or explored.

\subsubsection{Mitigation methods}

Various methods to mitigate core movement have been suggested over the years. Simple methods include reducing the core chamfer angle $\left(20^{\circ}\right.$ is standard) and using cores with higher relative density 
[10]. Owing to the severity of core movement, it is common for manufacturers to use a reduced pressure cycle of $310 \mathrm{kPa}$ (45 psi) gauge [8, 10, 35]. This is in contrast to traditional laminates which are typically processed around $621 \mathrm{kPa}(90 \mathrm{psi})$ gauge. In addition to reducing the external pressure, manufacturers often implement tie-down plies [12, 26, 29, 37, 40, 41], wherein specific plies are restrained from moving. This is one of the most common ways to prevent core movement. In this setup, generally plies nearest the core are tied-down. The idea being that if ply movement is prevented, then so is core movement - assuming the core does not deform relative to the over and underlying facesheets. Plies are often restrained through the use of grit strips (sharp strips along the tool that plies are adhere to) [37, 41], although adhesives have also been used [26]. Restraining occurs along the plies' outer edge, past the trim point of the panel.

Another industrial technique to combat core movement includes mechanical stabilization of the core $[10,13,17,35]$. Many variations on the process exist, with the idea being to increase rigidity of the core. In one scenario [13, 17], a foam adhesive is applied to the core chamfer and a film adhesive is applied along the flat underside of the core. The structure is then cured. The process, however, may take several hours and must be done prior to the sandwich panel layup [13]. Alternatively, it is common to implement a film adhesive layer only along the underside of the core and pre-cure it. Processes such as these may also be used to improve rigidity for the purpose of machining and are often referred to as Core Machining Stabilization (CMS). Septumization of the core has also been used to mitigate core movement [10,37] by increasing core rigidity; although this technique has largely been used to improve acoustical dampening properties of the honeycomb. Adding a septum involves sectioning the core and bonding pre-cured plies (typically glass fiber) within the center of the core. Finally, while potting is typically used to allow for mechanical fastners to be attached to the sandwich panel, it has also be employed as a means to strengthen the core in order to prevent core movement [10]. In the latter case, potting agent is added to select honeycomb cells and then cured to effectively increase the local core density.

Altering the cure cycle to allow for a build up of in-core pressure has also been shown to be an effective solution to managing to core movement [6, 8, 42]. The gas pressure entrapped within the core during cure increases with temperature according to the ideal gas law. Therefore, during the heating phase of the cure cycle resistivity to core collapse increases (assuming pressure is kept constant). Hence, core movement is most likely to occur early in the cure cycle. As such, Brayden and Darrow [8] suggest keeping the autoclave pressure low initially - 69 to $103 \mathrm{kPa}$ (10-15 psi) gauge - and increase it to 310 $\mathrm{kPa}$ once the temperature has reached a desired limit and in-core pressure has built up; typically the first hold temperature. In this design, vacuum pressure is initially reduced and then vented as pressure is raised. This allows for volatiles to escape during heating but prevents over pressurizing the panel once external pressure is increased. Working off this theory, Alteneder et al [6] showed that you could induce an initial pressure state in the core which prevents core movement from occurring early in the cure cycle. This is achieved by early pressurization of the vacuum bag to match the autoclave pressure. As long as the consolidation pressure is less than $125 \mathrm{kPa}$, the gas within the vacuum bag will permeate through the prepreg to the core. After some time, bag pressure is reduced to atmospheric conditions while autoclave 
pressure increases. This effectively seals off the core by increasing the consolidation pressure across the prepreg. Autoclave pressure can then be increased at a rate proportional to the expansion of gas within the core (during heating) such that the pressure differential between autoclave and core remains relatively low.

Aside from early work on core movement, much of the research has focused on improving the material system. It has been shown that the both resin viscosity and surface resin distribution affect core movement [28, 35, 37]. Although low viscosity systems may exhibit lower frictional resistance in the hydrodynamic regime, the resin can easily flow into interstitial zones, thus decreasing the amount of surface resin present and improving fiber intermingling. High viscosity systems present higher friction in the hydrodynamic regime but don't flow as well and are, therefore, typically coupled with high amounts of surface resin. As temperature increases, viscosity decreases and remaining surface resin may, therefore, act as a lubricant. As such, it has been suggested to avoid prepreg systems exhibiting the combination of high resin viscosity and high initial surface resin content [35]. Furthermore, reducing elastomer content in prepregs allows for a reduction in surface resin content during cure, leading to a subsequent increase in frictional resistance [37].

Pelton et al further showed that by twisting fiber tows, one can significantly improve the frictional resistance of the system [40]. Three kinds of tows were tested, namely, Never Twisted (NT), UnTwisted (UT), and Standard Twist (ST). The UT tows represented tows that were twisted and then untwisted. Both UT and ST tows demonstrated significant improvement in frictional resistance over NT tows, and eliminated or greatly reduced the severity of core movement. The use of ST tows has become common practice in some industrial settings. Hsiao et al [28] also showed that prepreg with rounder tows exhibit higher friction. Tow rounding is typically achieved by twisting or untwisting the tow. It can also be done by applying a chemical sizer or naturally occurs due to cross sectional conformations of the fibers. Rounder tows result in larger undulations and thus have a rougher surface. This in-turn creates a more open fabric allowing for improved fiber interlocking. Moreover, rounder tows showed a higher degree of impregnation, meaning less surface resin is present to act as a slip plane. Prepregs with varying tow aspect ratios and thicknesses were used in their experiments, with ubiquitous agreement that lower aspect ratio and greater thickness (i.e. a more round shape) hinders core movement. It was also shown that prepreg with larger tows ( $12 \mathrm{k}$ versus $3 \mathrm{k}$ ) were more susceptible to movement, but could be mitigated through tow rounding. Patents have been filed in utilizing such methods to create crush resistant fabric and prepreg for composite sandwich panels [27].

\subsubsection{Gas flow within the core}

While in-core pressure is important for reducing core movement, the application of vacuum will reduce entrapped gas unless specifically accounted for [8]. Honeycomb is generally considered impermeable in its in-plane dimension, if not perforated [46]. Therefore, gas must travel through or within the prepreg to escape [33, 46-48]. Gas is vented from the core in two ways. Either through the bagside laminate in the transverse (through-thickness) direction or out the edges of the panel in the in-plane direction. The latter occurs if edge breathing techniques are used and the former occurs if the release film is permeable 
or perforated [33]. Depending on the permeability of the adhesive and the compaction pressure along the panel edgeband, it can take anywhere from minutes to hours for gas to flow in and out of the core [6, 33, 47, 48]. Kratz and Hubert [33] showed that gas transport out of the core can be broken into three main events, namely: delay time, linear increase in air permeability, and final in-core pressure. Delay time refers to the time until in-core pressure begins to drop. The permeability then increases linearly as pressure continues to drop, until the pressure eventually stabilizes. The magnitude of each of these regimes showed large variations amongst equivalent experiments. In addition, different weave patterns greatly influenced permeability. Plain weave fabric showed the highest permeability in comparison with unidirectional and 5 harness satin weaves. The final in-core pressures of plain weave fabric panels held under vacuum for 24 hours varied between 5-20 kPa. It was also shown that permeability was significantly greater in-plane rather than in the through-thickness direction.

Travares et al [47] demonstrated that the delay time is a result of the film adhesive being impermeable prior to cure. Panels without film adhesive showed an immediate reduction of in-core pressure upon application of vacuum. Evacuation of in-core gas is significantly improved if the film adhesive is perforated. Traveres et al [48] also showed that the optimal range of in-core pressure for skin-core adhesion is 40-70 kPa. Similarly Kratz et al [32] showed that pressure fluctuations arise if the in-core pressure reaches $1 \mathrm{~atm}$ and may act to increase porosity. However, these experiments are for VacuumBag Only (VBO) processing of sandwich panels. Therefore, they do not necessarily contrast the desire for high in-core pressure to mitigate core movement during autoclave processing of sandwich panels.

Both Taveres et al [46, 47] and Kratz et al [32,33] used a similar setup to measure in-core pressure and related this to permeability. The setup involves the use of a cavity under the core connected to a pressure transducer. As a result, plies are only placed on the bagside of the core. The transducer measures the pressure in the cavity prior to cure and as cure develops. This is synonymous with in-core pressure for this setup. However, without the presence of toolside plies, each cell of the honeycomb core is effectively connected. This behaves similar to a core with perforated cell walls. Thus a pressure gradient cannot develop across the honeycomb, as may be the case in a "real" sandwich panel.

Alteneder et al [6] measured in-core pressure during autoclave processing of sandwich panels for a material system qualified to BMS8-256. In their experiments, bag-core pressure stabilization was achieved in around 15 minutes. The exact time depended on the adhesive system implemented and the compaction pressure. It was shown that the rate of gas flow across the panel edgeband achieved a steady-state value within 500 seconds for consolidation loads less than $125 \mathrm{kPa}$. Above $125 \mathrm{kPa}$ of compaction pressure the prepreg became impermeable in a matter of minutes, trapping gas within the core. 


\section{Chapter 3}

\section{Aims and Objectives}

Factors influencing core movement and their relation to each other were identified and a core movement map was created. Figure 3.1 provides an overview of the map. The layout is such that there are three main categories that influence core movement, namely: materials, core geometry, and processing parameters. Within these there are sub-categories that directly influence core movement. The map is not intended to provide an exhaustive list of all potential factors involved in core movement, but rather to summarize the major components along with those of interest to this body of work.

Much of the past research on core movement focuses on the materials aspect of the phenomenon. In particular, a great deal of work has been done on correlating the in-core pressure and frictional resistance of the prepreg system to core movement. However, there is a lack of knowledge in understanding the physical process of core movement. As a result, the impact of altering processing parameters in regards to core movement is not well defined. This similarly extends to alterations in geometry and materials as well.

The objectives of this body of work can be broken into two broad categories. Namely, to better understand the factors involved in core movement during processing and to construct an improved model of the phenomenon based on the experimental findings. This is not an attempt to provide a complete analysis of all potential factors in core movement, but rather provide clarity to the problem of core movement itself as well as analyze the effect specific parameters have on core movement.

The objectives of this research can be broken down as follows:

1. Develop a better understanding of the factors influencing core movement during processing of sandwich panels including:

- Effect of temperature/viscosity

- Effect of pressure

- Effect of various layups

2. Build a simple mechanical model based on experimental observations including:

- Initiation/progession of core movement 


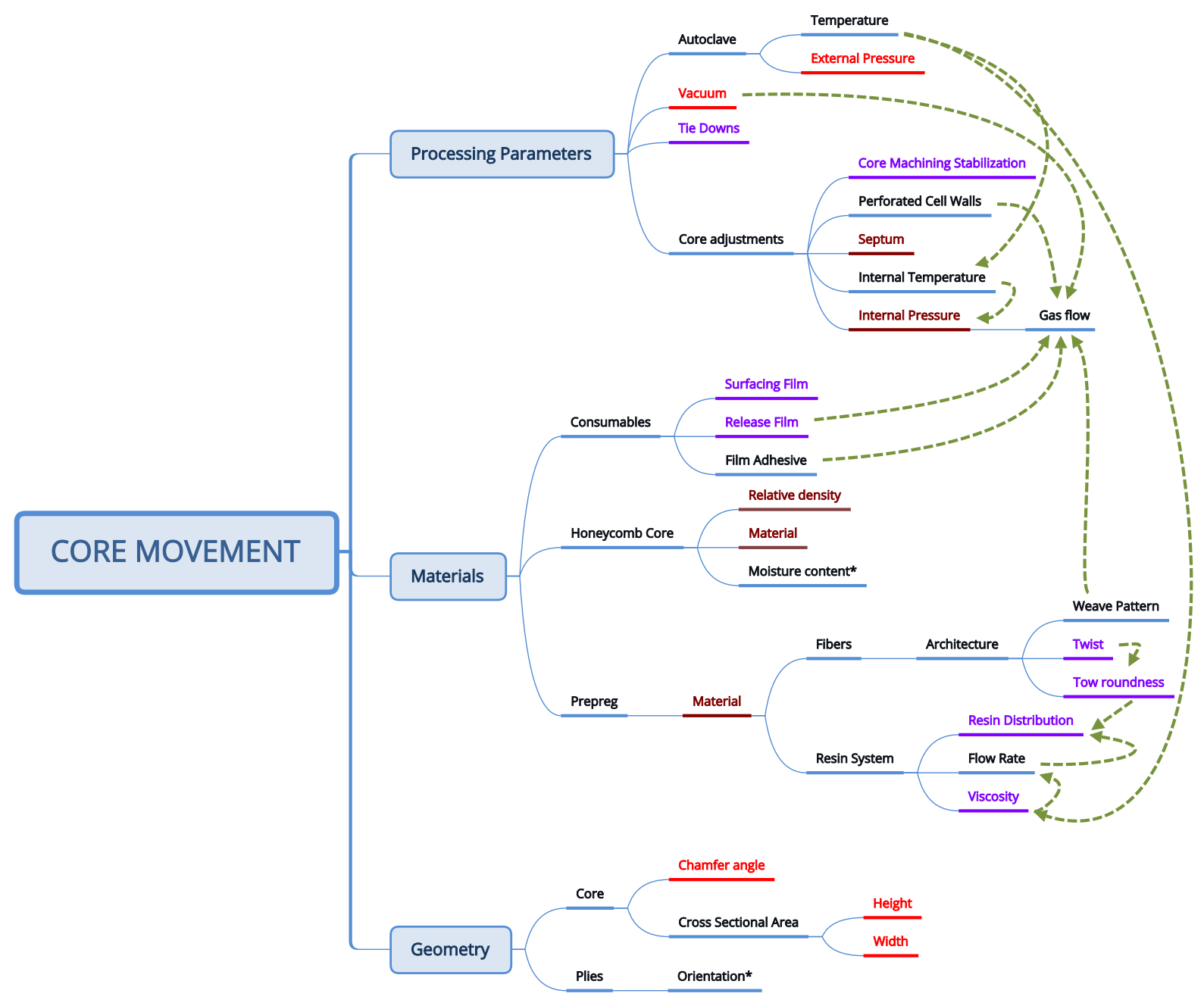

Figure 3.1: Core movement map. Major factors known to influence core movement are displayed. Green arrows indicate when one property influences another. Properties with an asterisk are suspected to influence core movement, but their relation to core movement has not yet been documented nor are they investigated in this body of work.

- Specific ply movement

- Effect of processing conditions

The aim of this research is to shed light on the core movement process so that manufacturers can implement science-based methods to combat the problem. The primary questions that are posed and addressed include:

- How does core movement occur?

- How/where does it initiate?

- What are the sequence of events?

- When in the processing cycle does core movement occur? 
- Is it temperature dependent?

- Does it occur below a certain viscosity?

- Do current models capture everything?

- What are the major forces at play?

Other specific questions that are addressed include:

- How effective are tie downs?

- How does core machining stabilization affect core movement?

- How does core type affect processing?

- Chamfer angle

- Ribbon vs non-ribbon direction 


\section{Chapter 4}

\section{Methodology}

\subsection{Experimental breakdown}

In order to effectively answer the questions posed in Chapter 3, a design of experiment was performed and ten individual tests were conducted. They can be broken into the following three broad categories:

\section{Effect of Temperature}

Temperature is increased to a set value, after which pressure is increased relatively slowly. Layup is equivalent among tests; only the set temperature differs.

- Pressure at core movement initiation is recorded

\section{Effect of Pressure}

Pressure is increased rapidly to a set value, after which temperature is increased relatively slowly. Layup is equivalent among tests; only the set pressure differs.

- Temperature at core movement initiation is recorded

\section{Effect of Layup}

Aspects of the layup are changed for each test. During processing, temperature is increased to $120^{\circ} \mathrm{C}$ and held constant. Thereafter, pressure is increased relatively slowly.

- Pressure at core movement initiation is recorded

In each test, the material system is kept constant. A summary of the experiments is listed in Table 4.1. Further details regarding the testing procedure is provided in the sections that follow. 
Table 4.1: Summary of experiments. Note that the $120^{\circ} \mathrm{C}$ and "Standard" experiment are the same test and represent the baseline test.

\begin{tabular}{|c|c|c|}
\hline $\begin{array}{c}\text { 1. Effect of Temperature } \\
\text { Standard layup } \\
\text { Set temperature } \\
\text { Increase pressure }\end{array}$ & $\begin{array}{c}\text { 2. Effect of Pressure } \\
\text { Standard layup } \\
\text { Set pressure } \\
\text { Increase temperature }\end{array}$ & $\begin{array}{c}\text { 3. Effect of Layup } \\
120^{\circ} \mathrm{C} \\
\text { Set layup } \\
\text { Increase pressure }\end{array}$ \\
\hline $25^{\circ} \mathrm{C}$ & $325 \mathrm{kPa}(47 \mathrm{psi})$ & Standard \\
\hline $95^{\circ} \mathrm{C}$ & $600 \mathrm{kPa}(87 \mathrm{psi})$ & Full single tie-down plies \\
\hline $120^{\circ} \mathrm{C}$ & & Half single tie-down plies \\
\hline $180^{\circ} \mathrm{C}$ & & Core machining stabilization \\
\hline & & $45^{\circ}$ core chamfer \\
\hline
\end{tabular}

\subsection{Materials}

The prepreg used comprised of Cycom 970 epoxy resin with T300 3k fibers from Toray. The fibers are plain weave, standard twist, carbon fabric. The nominal resin content is $40 \%$ by weight and contains added toughners. The material system is qualified to BMS 8-256. It is a low flow, high temperature cure $\left(177^{\circ} \mathrm{C}\right)$ material and is non self-adhesive, meaning a film adhesive must be placed between the prepreg and core. The film adhesive used was Metlbond 1515-4 which is co-cured with the prepreg and has the same Manufacturer Recommended Cure Cycle (MRCC). The core itself was HexWeb HRH-101/8-3.0 from Hexcel [25]. It is a regular hexagonal Nomex honeycomb core with a cell size of 3.175 $\mathrm{mm}$, density of $48.06 \mathrm{~kg} / \mathrm{m}^{3}$, and approximate cell thickness of $0.08 \mathrm{~mm}$. The chamfer angle of the core was $20^{\circ}$, except in the noted experiment where the chamfer angle was $45^{\circ}$. Finally, Surface Master 905 from Solvay was used as the surfacing film on the tool, to provide a good surface and improve resin distribution on the base of the panel. It is also co-cured with the prepreg and has the same MRCC. A 12 mm thick, A36 steel tool was used for the experiments. The tool included AISI 321 stainless steel grit strips adhesively bonded and riveted along the tool's perimeter to allow for plies to be tied down.

\subsection{Sensors}

Two DS1000SUH Linear Variable Displacement Transducers (LVDTs) were used to track deformation of the panel in-situ. These were coupled with a 5M30 AC LVDT Conditioner to provide a calibrated analog output signal for displacement. The sensors were placed normal to the chamfer edge or radius of the panels in the $\mathrm{L}$ and $\mathrm{W}$ direction. For the $120^{\circ} \mathrm{C}$ and $45^{\circ}$ chamfer experiments (described later), the LVDTs were placed along a single panel edge at the top and bottom of the chamfer in order to track the pattern of deformation of the chamfer itself. In each experiment, embedded pressure sensors were surgically placed in the core at the chamfer radius to track in-core pressure fluctuations throughout the entire process. This included measuring pressure in the core following vacuum application, as well as during core movement. Early experiments featured two pressure sensors. However, due to lack of resources, later experiments only featured one pressure sensor. Both bagside and toolside locations were tested for pressure sensor placement with no differences seen. Finally, an autoclavable camera was setup 


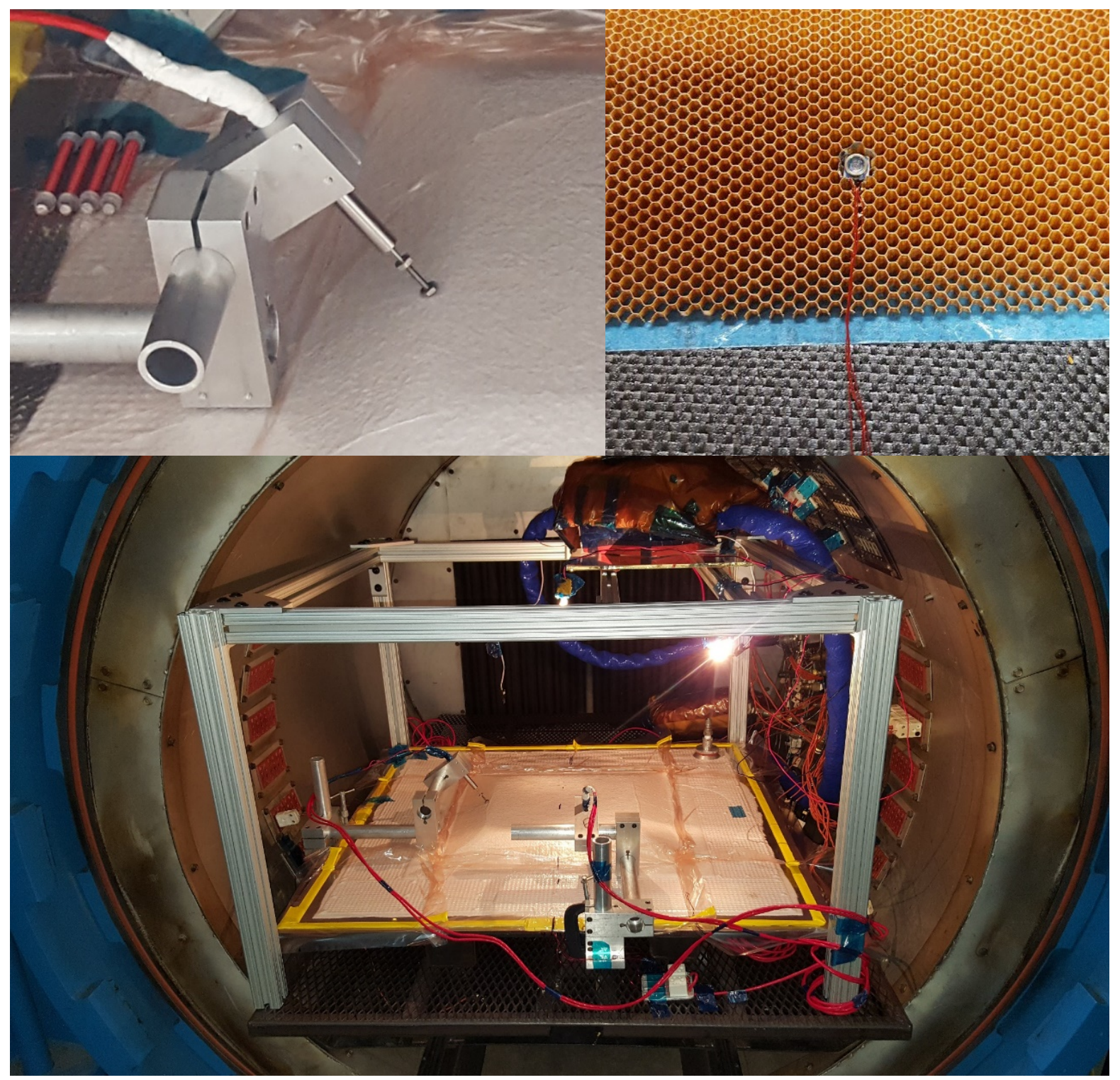

Figure 4.1: Test setup showing in-situ sensors. Top left is an LVDT sensor, top right is an in-core pressure sensor, and the bottom is the full setup in the autoclave with the camera mounted above the panel.

to allow for real-time visualization of the core movement process. Due to the size of the panels and field of view of the camera, the recording was restricted to one corner of the panel. Both the pressure sensors and camera were developed by Convergent Manufacturing Technologies Inc. The sensor setup is shown in Figure 4.1. More information on the in-situ sensors and camera setup are provided in Appendix A.

For the Dry Core test (described in Section 4.8) a GoPro was used to record the process, allowing for the entire core to be captured. The same setup could not be implemented in tests involving heating due to operating restrictions of the GoPro. 


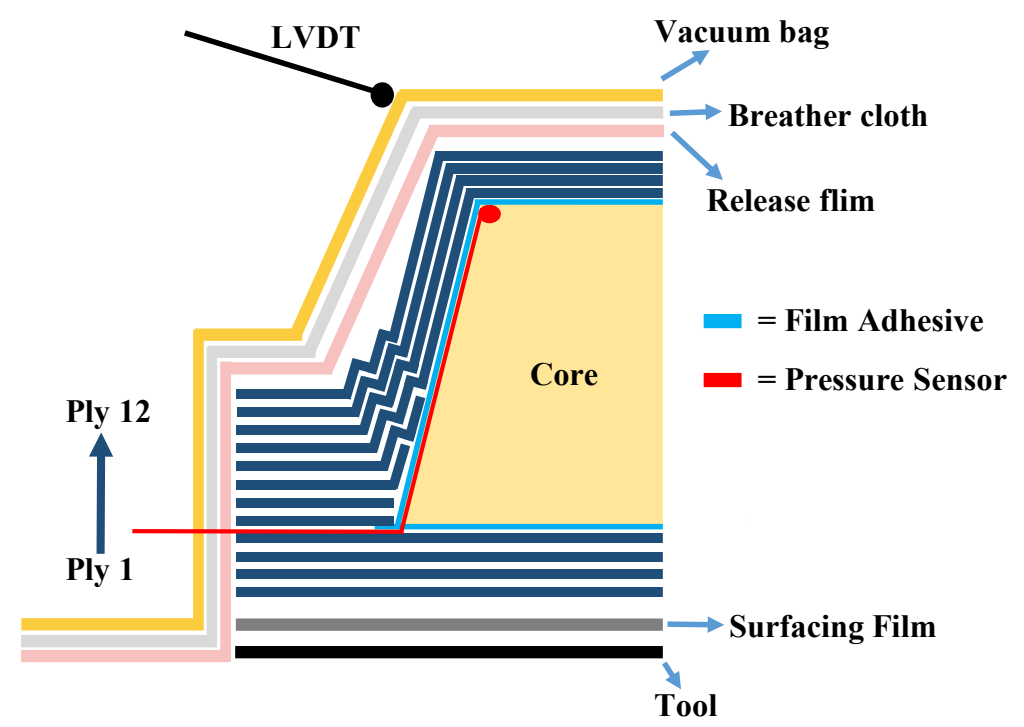

Figure 4.2: Standard sandwich panel layup. First layer against the tool is surfacing film, followed by 4 toolside plies. Core with pressure sensor and film adhesive layers is then added, followed by 4 filler plies. Next, 4 bagside plies are draped over the core. PTFE release film, breather, and vacuum bagging are then added. The LVDT; are placed after vacuum bagging is complete.

\subsection{Layup}

As laid out in Table 4.1, the experiments consisted of three distinct categories, 1 - effect of temperature, 2 - effect of pressure, and 3 - effect of layup. In category 1 and 2, the layup for each experiment was identical and is referred to as the standard layup. In category 3 , however, the layup was varied for each experiment in order to study its effect on core movement.

\subsubsection{Standard layup}

Figure 4.2 provides an illustration of the general layup used. Each panel consisted of a layer of surfacing film, twelve individual prepreg plies, one core, and two layers of film adhesive. The surfacing film was the first layer against the tool, followed by four prepreg plies (plies 1-4). Film adhesive was then centered on the laminate and the core was centered on the film adhesive. Pressure sensors were inserted in the core and then another layer of film adhesive was draped over the core. Four layers of filler plies (plies 5-8) were placed around the core in a pinwheel design, to allow for a smooth edgeband transition. The first two filler plies (plies 5-6) were butted against the core with the following two plies (plies 7-8) extending $6.35 \mathrm{~mm}$ and $12.7 \mathrm{~mm}$ up the core edge respectively. Four, full length prepreg plies (plies 9-12) were draped over the whole structure. The film adhesive layers extended $12.7 \mathrm{~mm}$ past the core edge. Ply 1 was held under vacuum for five minutes after being laid down to prevent shifting during layup.

Figure 4.2 shows the pressure sensor on the bagside surface of the core. As mentioned, the toolside location, directly under the radius of the core, was also tested with no observed difference of in-core 
pressure as compared with the bagside location. For pressure sensors placed on the bagside surface, A small cutout in the upper film adhesive around the sensors were made to prevent intrusion of the film into the sensors during cure. For both toolside and bagside locations, the walls of the honeycomb cells were cut along the length of the pressure sensor cables to allow the cables to sit firmly in the core, thus preventing shifting of the sensor itself and the allowing for plies to be laid flat.

Once the layup was finished, Polytetrafluoroethylene (PTFE) release film was placed on the prepreg and breather cloth surrounded the entire panel. The part was then vacuumed bagged with pleats extending along the edgeband transitions to prevent bridging. Exact debulk time was not measured, but was limited to a few minutes.

As mentioned in Section 4.3, the $120^{\circ} \mathrm{C}$, standard layup and $45^{\circ}$ chamfer experiment (described below) featured both LVDTs on the same panel edge, with the second sensor positioned against the base of the chamfer. This is not shown in Figure 4.2. Moreover, a $12.7 \mathrm{~mm}$ x $12.7 \mathrm{~mm}$ grid pattern was drawn on the breather cloth to track the rate of deformation in the video footage. These two panels were chosen as representative tests for investigating the rate of deformation in panels with varying chamfer angles, namely $20^{\circ}$ and $45^{\circ}$.

\subsubsection{Specific layups}

Four specific layups were tested to observe their effects on core movement. They include, full single tie-down plies, half single tie-down plies, CMS, and use of a core with a $45^{\circ}$ chamfer angle. The layups follow the same gross procedure as for the standard layup, with minute differences in each. The differences are described below.

\section{Full single tie-down plies:}

The term "single" refers to the number of plies above and below the core that are tied-down. Single tie-down, therefore, refers to a layup in which one toolside and one bagside ply are restrained. In this experiment, ply 4 and ply 9, as shown in Figure 4.2, are restrained. These represent the nearest full plies (i.e. not filler plies) to the core. The plies are restrained by tacking the plies onto the grit strips surrounding the tool. All four edges of each ply are restrained, hence the term "full tie-down". Since ply 4 is placed first, it extends $25.4 \mathrm{~mm}$ onto the grit strips. To allow ply 9 to have an equivalent "fresh" section of grit strip, it extends a further $25.4 \mathrm{~mm}$ past ply 4 , or $50.8 \mathrm{~mm}$ total, onto the grit strip. The remainder of the layup is identical to that described in Section 4.4.1.

\section{Half single tie-down plies:}

This layup follows the exact same procedure as that for full single tie-down plies, with one difference. Only two edges of plies 4 and 9 are restrained. Two adjacent edges are restrained such that a ribbon and non-ribbon edge are restrained and the other ribbon and non-ribbon edges are free to move. A pressure sensor was placed on both a restrained and unrestrained non-ribbon edge. 

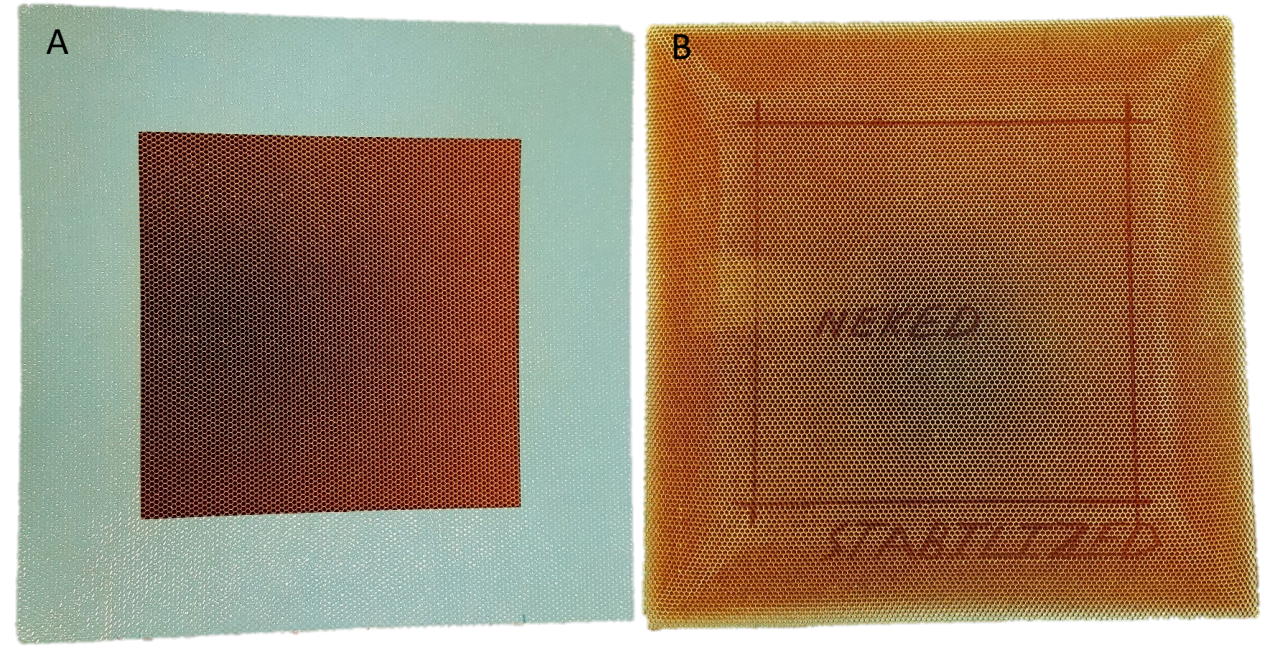

Figure 4.3: Core machining stabilization - $76.2 \mathrm{~mm}$ wide picture frame film adhesive strip is cured along the toolside of the core. (A) Toolside, (B) Bagside.

\section{Core Machining Stabilization (CMS):}

Prior to layup, CMS is performed (see Figure 4.3). A $76.2 \mathrm{~mm}$ wide picture frame strip of film adhesive is cut out and placed around the toolside perimeter of the core. This is then cured under reduced vacuum (27.1 - $33.9 \mathrm{kPa}$ ) according to the MRCC, with no external pressure applied to prevent core movement. A layer of PTFE release film was placed under the film adhesive during cure in order to roughen up the surface and provide a good bond during layup. The center of the core remained unstabilized or "neked". Once stabilized, the core is then used in the layup as described in Section 4.4.1. During layup, film adhesive is still placed under the stabilized core, to provide the necessary bond with the underlying prepreg facesheet.

\section{$45^{\circ}$ chamfer:}

In this scenario, the layup follows that of the standard layup described in Section 4.4.1. However, a honeycomb core with a chamfer angle of $45^{\circ}$ was used instead of one with a $20^{\circ}$ chamfer angle.

\subsection{Cure cycle}

Similar to layup, the cure cycle varied depending on the specific experiment. The general procedure was such that the part was heated until reaching a set temperature after which pressure was applied, with the exception of category 2 experiments according to Table 4.1. The cure cycle for category 3 experiments is referred to as the standard cure cycle. Category 1 experiments largely follow this cure cycle with the main difference being that the hold temperature varies for each experiment. It should be noted that the cure cycles presented in 4.4 are different from the MRCC, as the purpose of these cycles are to isolate their effects in relation to core movement. RAVEN models [3] were used to calculate the resin viscosity of each sample throughout the cure cycle. 


\subsubsection{Standard cure cycle}

Heating was applied at a rate of $5^{\circ} \mathrm{C} / \mathrm{min}$, until the temperature reached $120^{\circ} \mathrm{C}$, after which pressure application began. External pressure was set to increase at a rate of $21 \mathrm{kPa} / \mathrm{min}$, however the autoclave controller could not achieve this. As a result, a stepwise pressure cycle was introduced to attain a stable response. Autoclave pressure was ramped up in steps of $103 \mathrm{kPa}(\approx 1 \mathrm{~atm})$ up to $600 \mathrm{kPa}$ (87 psi) gauge. After each step, pressure was held for one minute to artificially induce slow pressurization. Once maximum pressure was achieved, it was held for three hours while the part cured. At $103 \mathrm{kPa}$ gauge, the vacuum bag was vented to atmospheric pressure to prevent additional force acting on the panel and reduce the chance of core movement occurring too early. $120^{\circ} \mathrm{C}$ was chosen as the standard processing temperature as it is around this temperature when Cycom 970 reaches minimum viscosity under the MRCC.

\subsubsection{Specific cure cycles}

\section{Room temperature test}

A single test was conducted at room temperature to observe the effects on core movement when no heating is applied. This is listed as the $25^{\circ} \mathrm{C}$ experiment under Table 4.1. Since this was the first test conducted, the pressure cycle was not altered to achieve slow pressurization. As a result, pressure initially increased around two times faster than the standard cure cyle described above. Once the external pressure reached $420 \mathrm{kPa}$ gauge, however, the rate of pressurization slowed considerably. No heating was applied, however the temperature within the autoclave rose to approximately $30^{\circ} \mathrm{C}$ due to pressurization. The remainder of the cure cycle followed the standard cure cycle outlined above.

\section{Effect of temperature:}

Three panels were built according to the standard layup. Each were processed at different temperatures, namely $95^{\circ} \mathrm{C}, 120^{\circ} \mathrm{C}$, and $180^{\circ} \mathrm{C}$. The $120^{\circ} \mathrm{C}$ sample followed the standard cure cycle described above. The other two samples also followed the same cure cycle but with different hold temperatures, i.e. $95^{\circ} \mathrm{C}$ and $180^{\circ} \mathrm{C}$ respectively. In these tests, after one minute at maximum pressure, the hold temperature was changed to $120^{\circ} \mathrm{C}$ for final cure.

The $180^{\circ} \mathrm{C}$ test was chosen as that is similar to the hold temperature listed under the MRCC. Moreover, $180^{\circ} \mathrm{C}$ is representative of high temperature processing for sandwich panels, while $120^{\circ} \mathrm{C}$ is indicative of low temperature processing. A test at $95^{\circ} \mathrm{C}$ provides an additional data point outside the typical processing window. For both $95^{\circ} \mathrm{C}$ and $120^{\circ} \mathrm{C}$ tests, the viscosity remains low after reaching its respective minimum; thereby maximizing the potential for core movement. Additionally, as shown in literature, frictional resistance reaches a minimum before viscosity does. $120^{\circ} \mathrm{C}$ represented the temperature at minimum viscosity under the MRCC. Therefore, it was hypothesized the frictional resistance could be lower at $95^{\circ} \mathrm{C}$. The viscosity profiles of the three samples are shown in Figure 4.4. It should be noted that at $180^{\circ} \mathrm{C}$, the sample begins to cure rapidly after reaching minimum viscosity.

In an early attempt to prevent rapid pressurization, an experiment was performed at $120^{\circ} \mathrm{C}$ following 


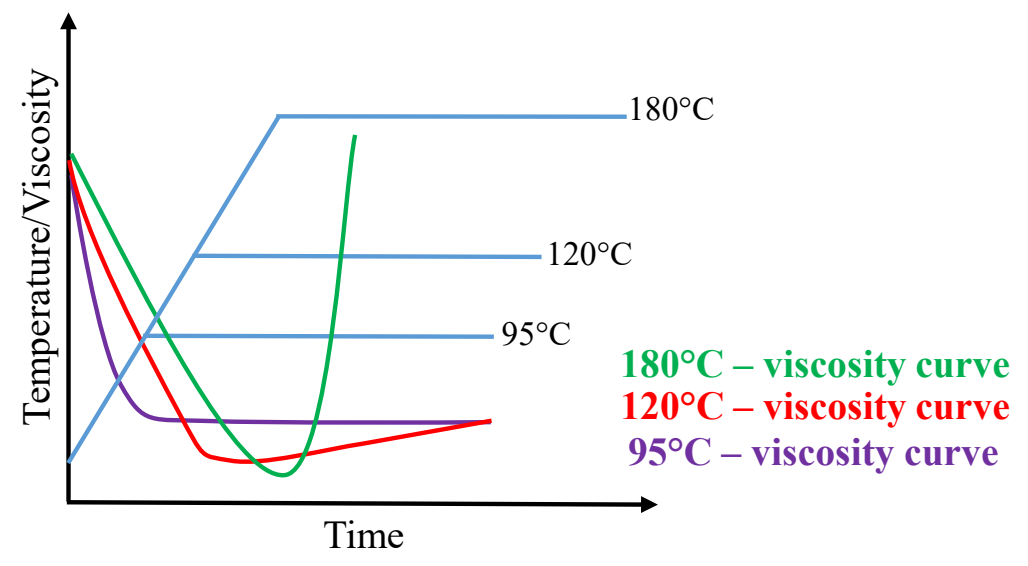

Figure 4.4: Representative viscosity profile for three different temperatures during cure. Adapted from RAVEN data received from M. Shead (Boeing Winnipeg).

the standard layup. It is herein referred to as $120^{\circ} \mathrm{C}_{\text {orig. }}$. In this test, a pressure hold of one minute was introduced at an autoclave gauge pressure of $325 \mathrm{kPa}$. Rapid pressurization still occurred however, paving the way for the introduction of the standard cure cycle outlined in Section 4.5.1. Other than extracting patterns in the deformation of the $\mathrm{L}$ and $\mathrm{W}$ edges, the results of this test are dismissed. They are presented in Appendix C.

\section{Effect of pressure:}

Two tests were conducted at different pressures, namely $325 \mathrm{kPa}$ (47 psi) gauge and $600 \mathrm{kPa}$ (87 psi) gauge. Pressure was applied rapidly $(\approx 172 \mathrm{kPa} / \mathrm{min})$ until the set pressure, after which heating began. Heating and the remainder of the cure cycle followed that outlined in Section 4.5.1. It should be noted that rapid pressurization induced notable heat in the system prior to external heating.

These tests are more indicative of standard sandwich panel processing in the sense that pressure is applied before the part is heated. $325 \mathrm{kPa}$ is typical for processing of sandwich panels, while $600 \mathrm{kPa}$ is representative of pressures used in the processing of laminates.

\subsection{In-situ data}

Data from the LVDT and pressure sensors were recorded real time using LabVIEW and later analyzed with MATLAB. A typical output is shown in Figure 4.5. Note that only one LVDT and pressure sensor is shown for clarity. The sudden early rise in vacuum pressure corresponds to venting of the vacuum bag. This is followed by a rise of in-core pressure. Considerable noise is often seen in the pressure sensors once deformation is apparent due to pulling of the wires as the core moves inward. In-situ sensor data and video footage were synchronized to allow for an understanding of the exact processing conditions during the entire core movement window.

The test involving full tie downs displayed no visible core movement. As such, the deformation seen in this test was taken to represent deformation of the breather cloth. It was assumed this breather deformation was indicative of the breather deformation for all the tests. This allowed for a decoupling 


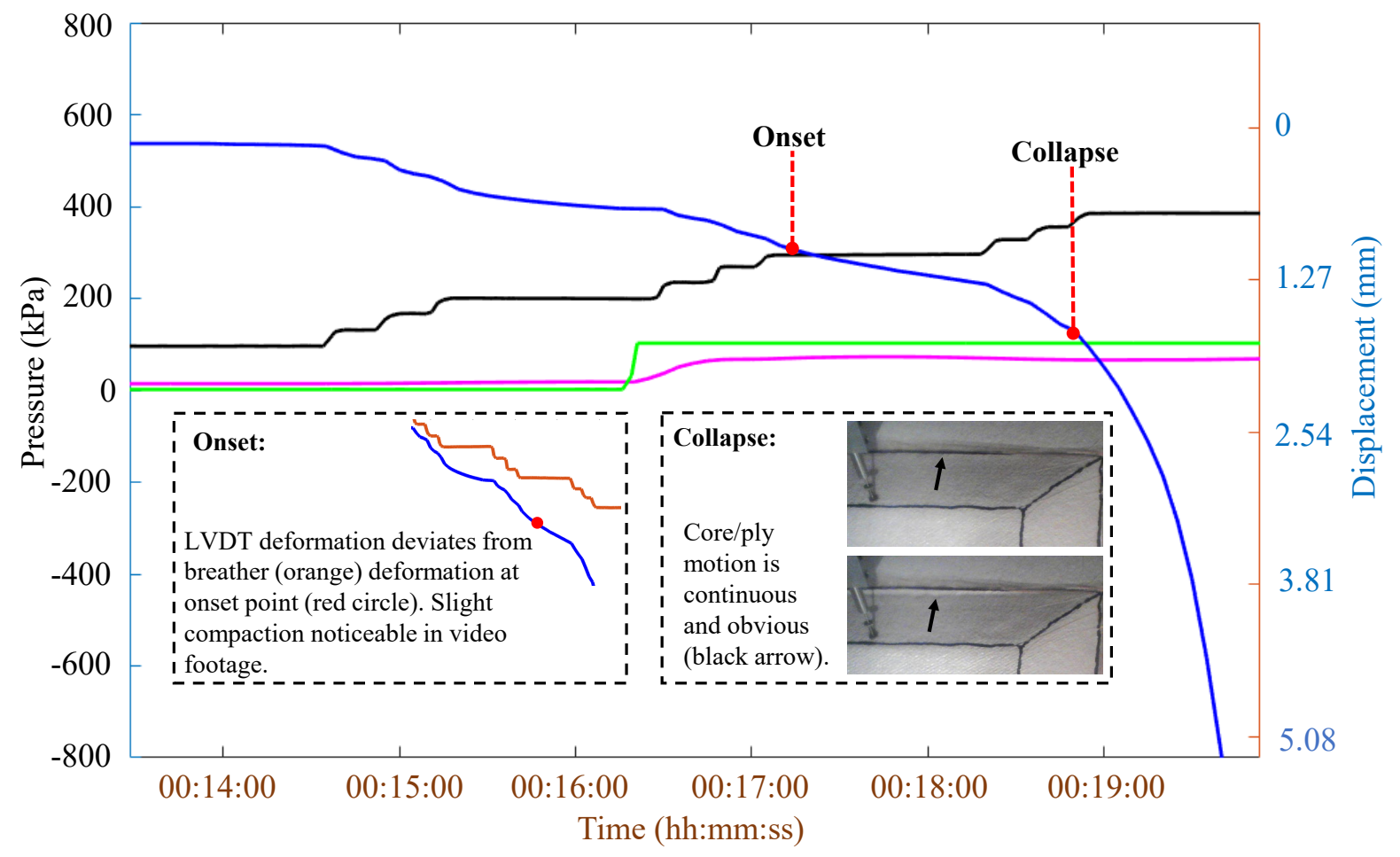

Figure 4.5: Data output from in-situ sensors. The colour scheme is as follows: black - autoclave pressure, blue - LVDT sensor, purple - in-core pressure sensor, green - vacuum pressure. The region between onset and collapse represents the critical zone, wherein core movement initiates.

of core movement from breather displacement for each experiment.

Core movement was broken into two stages, onset and collapse. These correspond to elastic bending and collapse of the honeycomb structure, respectively, as shown in Figure 2.3. Onset was defined as the first point of observable movement in both the video footage and LVDT data. This is represented in the LVDT data as the location where the displacement pattern of the panel deviates from that of the breather cloth. This manifests either as an increase in rate of panel deformation compared to the breather cloth or continued panel deformation while the breather deformation flatlines. In the video footage, the panel surface becomes notably rougher. Collapse was defined as the point of obvious, continuous deformation. That is, it is undeniable from video footage and LVDT data that core movement is occurring. Following collapse, the deformation proceeds rapidly at a relatively constant rate, independent of pressure holds. The transition is marked by a sharp increase in slope in the LVDT data and movement of core and plies are apparent in the video footage.

The absolute net pressure acting on the panels at the moment of onset and collapse were determined for each sample. This was in turn correlated with the viscosity of each sample at the exact moment of onset and collapse. This was done by matching the location on the viscosity profile, Figure 4.4, with the known time at which core movement occurs from the in-situ data. The range of pressure between onset and collapse is referred to as the "critical zone", as it represents the range over which core movement 
initiates. Pressures exceeding the upper bound of the critical zone are likely to result in drastic core movement, whereas core movement may be prevented if pressure is kept below the lower bound.

\subsection{Post cure data}

Following cure, panels were scanned using a Coordinate Measuring Machine (CMM) to obtain the surface topography. MATLAB was used to analyze the generated Point Cloud Data files. From this, the chamfer angle and panel height after core movement could be obtained. Furthermore, thickness changes across the samples were tracked to get an idea of ply movement. This was done for each sample. The full tie down test is represented as the control test, where no obvious ply movement is observed. Various regions along the panels were identified and labeled. Specifically, the panel edge refers to the outside edge of the panel, the core edge refers to the outside edge of the core at the base of the chamfer, and the edgeband refers to the plies immediately surrounding the core. It is representative of the region where all plies are located and is the thickest region of the panel excluding the core. Figure 4.6 provides a visual reference. The entire region between the panel edge and core edge is herein referred to as the laminate edge, as the plies in this area are effectively stacked as a laminate.

In addition to scanning, the panels were sectioned through-thickness along the $\mathrm{L}$ and $\mathrm{W}$ direction using a vertical bansaw with a diamond blade cutter. The cut samples were polished with sandpaper of varying grit. This allowed for individual plies along the laminate edge to be observed via microscopy. Several images of each sample were taken using a Keyence digital microscope and stitched together along the entire laminate edge. From these images, the location of each ply was tracked, so that individual ply movement could be determined as shown in Figure 4.7. It should be noted that individual plies often seem to disappear only to reappear later. This is due to plies moving in and out of the plane of the cut, either due to the cut not being perfectly straight or waviness associated with the tows themselves. In these cases, opposing tows (i.e. those in the out-of-the page direction) are used to help track the plies.

Through-thickness cuts also allowed for specific features of interest to be identified and labeled, such as the "crush zone". The crush zone represents the region of the core that undergoes deformation during core movement. It is best observable from a through-thickness section cut as shown in Figure 4.6. Additionally, wrinkle patterns in the bagside and toolside plies were more obvious from a throughthickness perspective.

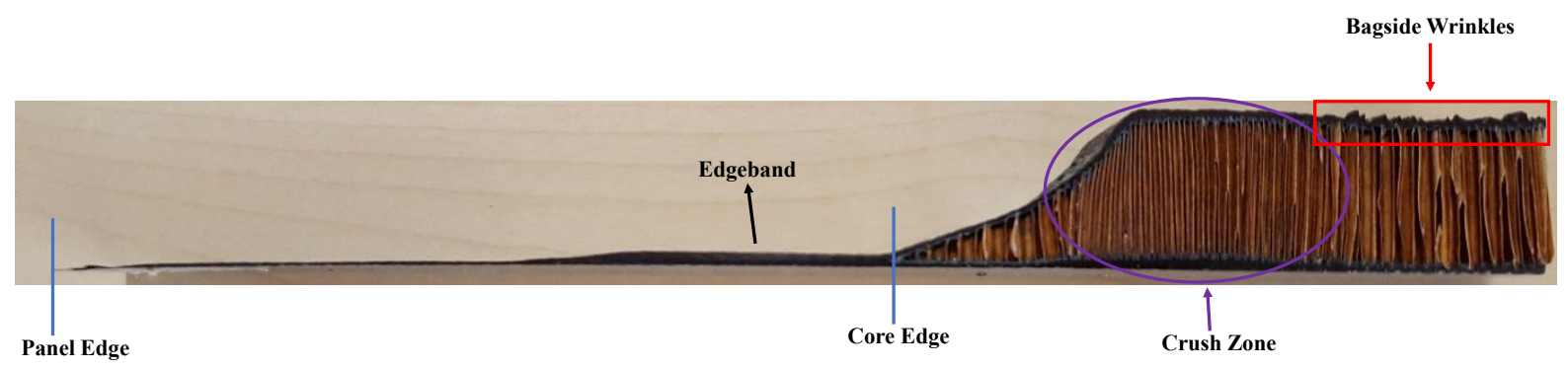

Figure 4.6: Polished through-thickness section cut. The region between the panel edge and core edge is referred to as the laminate edge. 


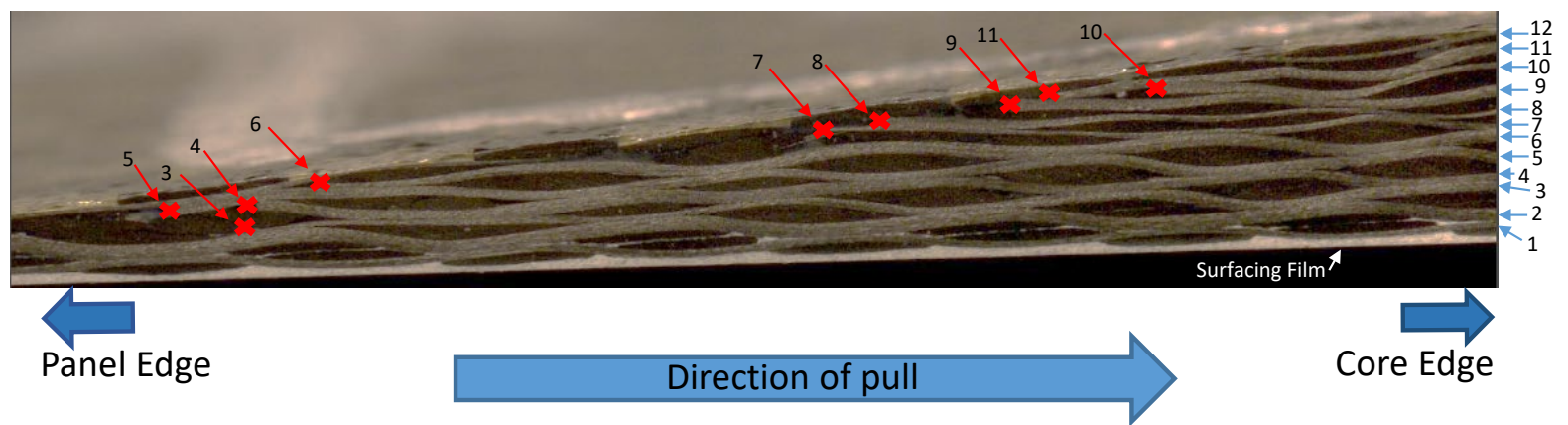

Figure 4.7: Cross sectional image of laminate edge showing individual plies and where they terminate after core movement. Numbers on the right identify the individual plies while the red Xs indicate where the associated ply terminates.

Several in-plane section cuts were also made using a vertical bansaw with a diamond blade cutter. The cut sections were polished with sandpaper of varying grit to allow for the cell deformation pattern to be observed. The difference in deformation pattern associated with compression in the $\mathrm{L}$ and $\mathrm{W}$ direction is of particular interest. Figure 4.8 provides a visual reference. 


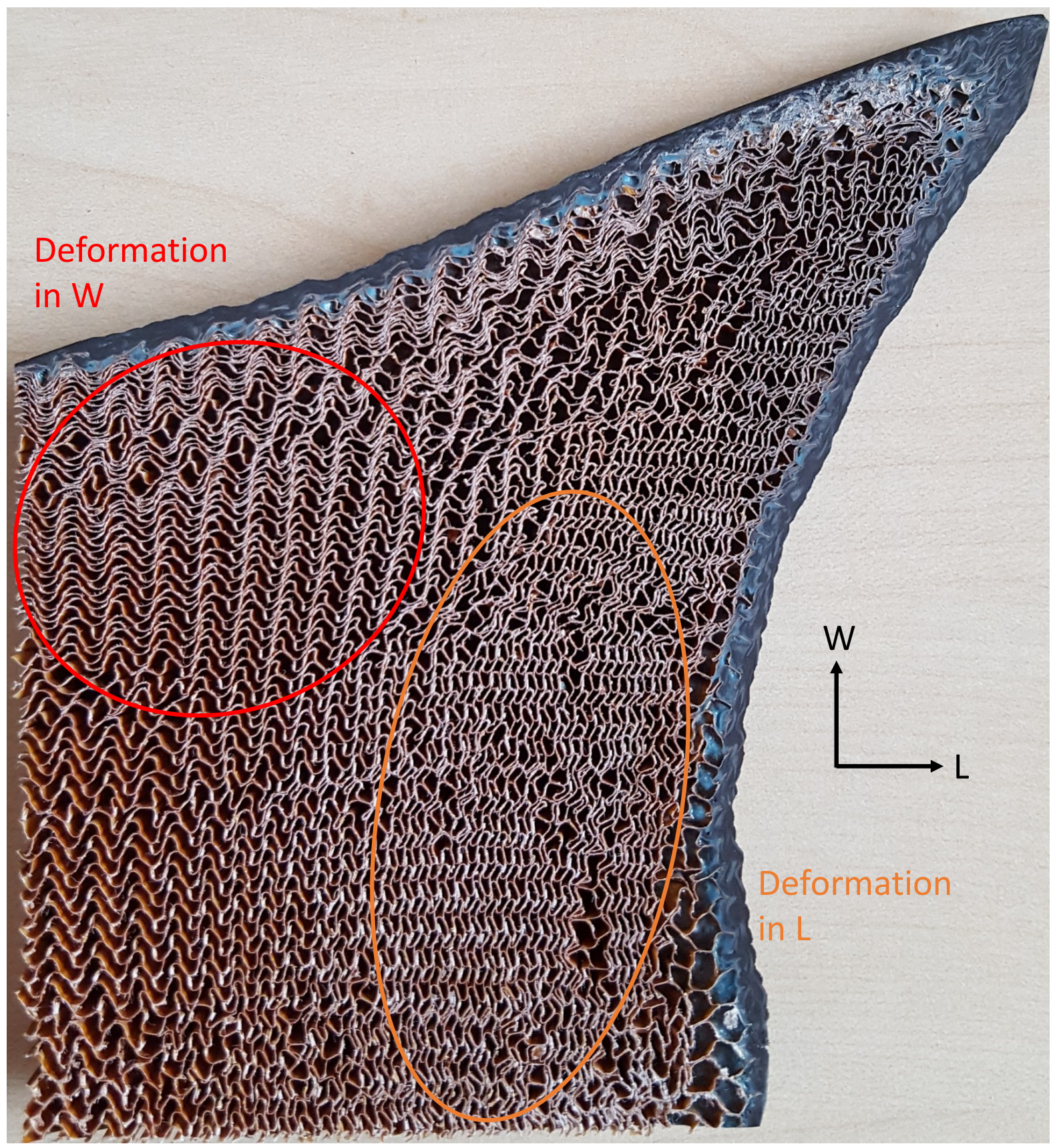

Figure 4.8: Polished in-plane section cut showing the deformation profiles in L and $\mathrm{W}$. 


\subsection{Dry core test}

A separate test was run where dry core (i.e. without plies) was placed in the autoclave. In this setup, breather cloth was only placed around the edges of the core. Release film was placed under the core to reduce friction effects with the tool. Other than the vacuum bag, nothing was placed on the bagside of the core to allow for visualization of the structure as it deformed. Only one LVDT was implemented as the other failed.

Pressure was applied according to the standard cure cycle outlined in Section 4.5.1. No heating was applied, however temperature rose to $38^{\circ} \mathrm{C}$ due to pressurization. A GoPro was mounted to the ceiling of the autoclave to film the process. 


\section{Chapter 5}

\section{Results and Discussion}

\subsection{Effect of temperature}

The following section details the effect temperature has on core movement. This includes both the initiation of core movement as well as the manner in which it progresses. Tests conducted include those listed under "Effect of Temperature" in Table 4.1.

\subsubsection{Failure pressure}

The absolute autoclave pressure at the moment of onset and collapse (as defined in Figure 4.5) was recorded for each specimen. The associated vacuum pressure at these times was subtracted from the autoclave pressure to give the pressure differential across the vacuum bag. This is the net pressure acting on the panels and represents the driving force for core movement initiation.

Figure 5.1(a) shows the net pressure at onset and collapse for each of the samples. The room temperature test displayed no core movement and is therefore not included in the figure. This indicates, that some level of heat application is required to cause core movement. Specifically, the external temperature must be greater than $25^{\circ} \mathrm{C}$ to result in noticeable core movement.

It is not temperature itself that is the root cause of core movement initiation. Rather, temperature influences properties of the resin, such as viscosity and ability to flow, which in turn influence core movement. Moreover, the length of time at which the sandwich panel is held at a given temperature also influences these properties. As such, the resin viscosity at onset and collapse is shown in Figure 5.1(b) to give a direct indication of the resin properties at the time of failure. It should be noted that from the time of pressure application through to initiation of core movement, the viscosity does not change for the $95^{\circ} \mathrm{C}$ and $120^{\circ} \mathrm{C}$ samples. However, the degree of cure (DOC) advances quickly for the $180^{\circ} \mathrm{C}$ sample, resulting in a rapid increase in viscosity. At the time of pressure application the $180^{\circ} \mathrm{C}$ sample had a viscosity of $12 \mathrm{~Pa}$.s. A few minutes later, the viscosity rose to $68 \mathrm{~Pa} \cdot \mathrm{s}$ during the onset of core movement, and was around $368 \mathrm{~Pa} \cdot \mathrm{s}$ by the time collapse occurred. These values are based on the numerical simulations from the RAVEN software and are best visualized in Figure 4.4.

The net pressure at onset is similar amongst the $95^{\circ} \mathrm{C}$ and the $180^{\circ} \mathrm{C}$ samples. The $95^{\circ} \mathrm{C}$ sample 


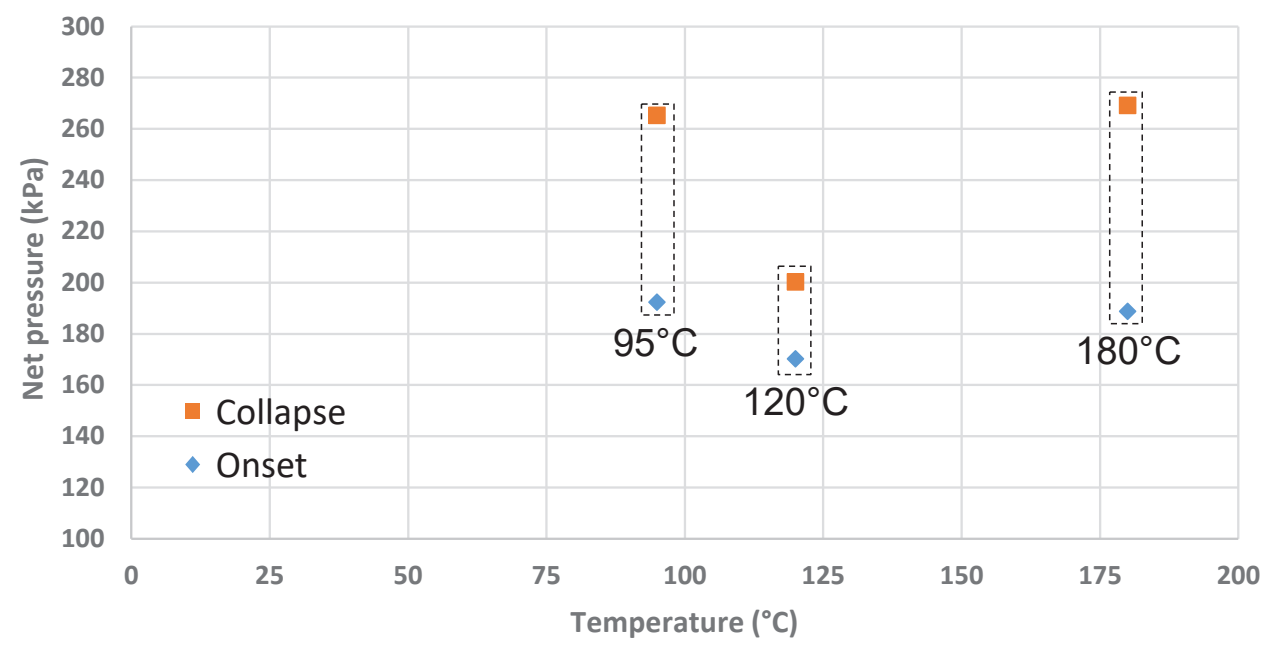

(a) Net pressure at failure for various processing temperatures

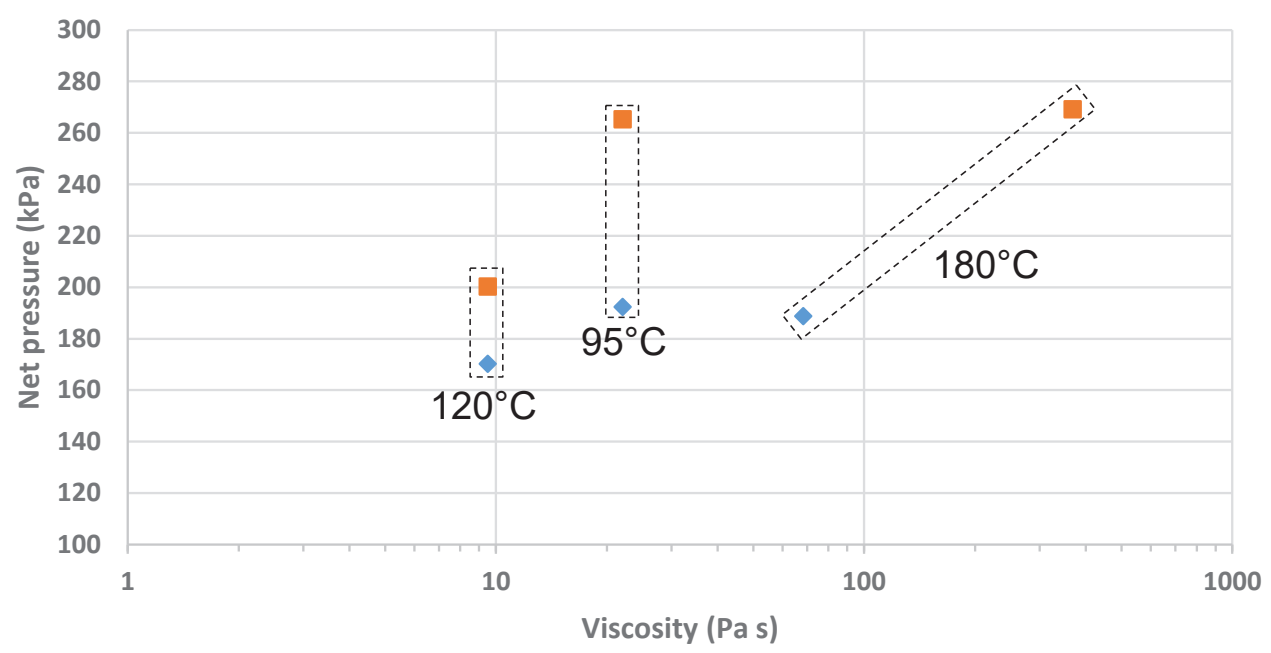

(b) Resin viscosity at failure

Figure 5.1: Net pressure acting on the sandwich panels at onset and collapse for the given (a) processing temperature and (b) associated resin viscosity.

displayed onset at $192 \mathrm{kPa}$ and the $180^{\circ} \mathrm{C}$ sample at $189 \mathrm{kPa}$. However, at $120^{\circ} \mathrm{C}$ the pressure to cause onset drops slightly to $170 \mathrm{kPa}$. This difference becomes more apparent at collapse, where the $95^{\circ} \mathrm{C}$ and $180^{\circ} \mathrm{C}$ samples fail at 265 and $269 \mathrm{kPa}$ respectively, yet the $120^{\circ} \mathrm{C}$ sample fails at $200 \mathrm{kPa}$. In addition to the lower failure pressure, the $120^{\circ} \mathrm{C}$ sample shows a much tighter critical zone of $30 \mathrm{kPa}$. Pressure fluctuations are, therefore, of concern as even small deviations of pressure could push the operating pressure from below the onset point up to collapse. In contrast, the critical zone of the $95^{\circ} \mathrm{C}$ and $180^{\circ} \mathrm{C}$ samples are 73 and $80 \mathrm{kPa}$ respectively. This is important for manufacturers as a larger critical zone allows for less stringent operating conditions.

The divergent behaviour of the $120^{\circ} \mathrm{C}$ sample is associated with a drop in viscosity below $10 \mathrm{~Pa} \cdot \mathrm{s}$ (as seen in Figure 5.1(b)). Interestingly, the $95^{\circ} \mathrm{C}$ and $180^{\circ} \mathrm{C}$ samples display markedly similar results in regards to failure pressure despite significant differences in their viscosity. This suggests that viscosity 
does not play as much of a role in resisting core movement at high temperatures; likely as a result of the surface resin distribution.

Since the prepreg system is constant amongst samples, the initial surface resin distribution is equivalent. Therefore, differences in surface resin distribution must come about during cure. Two factors contributing to the amount of surface resin are the ability for the resin to flow out under pressure and the rate at which the resin migrates from the surface to the core of the tows. The former is influenced by the resin viscosity while the latter is a product of the cure cycle. Since the $120^{\circ} \mathrm{C}$ sample displayed a lower resin viscosity at failure, it is reasonable to assume the surface resin content would similarly be reduced due to the ease of flow. However, this would act to increase the frictional resistance not decrease it. Therefore, this is perhaps not the case. The resin viscosity actually reaches a minimum in the $180^{\circ} \mathrm{C}$ sample, although this occurs prior to the application of pressure. Therefore, it is possible that the surface resin content of the $180^{\circ} \mathrm{C}$ sample was reduced in comparison with the other two samples due to resin migration into the core of the tows. This may explain its higher resistance to crush in comparison with the $120^{\circ} \mathrm{C}$ sample. It would also explain why viscosity has no apparent affect in regards to failure pressure between the $95^{\circ} \mathrm{C}$ and $180^{\circ} \mathrm{C}$ samples, because the $180^{\circ} \mathrm{C}$ sample is in a state of boundary lubrication dominated by dry, coloumb friction. The $95^{\circ} \mathrm{C}$ sample on the other hand displayed the highest resin viscosity throughout heating and therefore likely also contained the highest surface resin content. In short, the sample remained in a state of hydrodynamic lubrication. Therefore, its relatively "high" viscosity increased its frictional resistance to crush. It is possible that the $95^{\circ} \mathrm{C}$ sample exhibited hydrodynamic friction equal in magnitude to a boundary lubrication mode presented by the $180^{\circ} \mathrm{C}$ sample; hence their apparent similar resistance to core movement. The $120^{\circ} \mathrm{C}$ sample would then lie in between the others, i.e. considerable surface resin content coupled with a low resin viscosity resulting in reduced frictional resistance.

Figure 5.1 (a) implies that resistance to core movement follows a similar trend to inter-ply friction. That is, early on friction decreases with viscosity but eventually fiber-fiber contact dominates the response and friction stabilizes or increases slightly. The transition to a coloumb dominated response typically occurs before minimum viscosity [35]. However, as seen in Figure 5.1 (b), the lower failure pressure exhibited during the $120^{\circ} \mathrm{C}$ test seemingly corresponds to minimum viscosity, indicating hydrodynamic lubrication remains dominant up to this point. It is suspected, however, that beyond this point, viscosity would continue to decrease yet resistance to movement would in fact increase due to fiber intermingling. This would support the notion that the $180^{\circ} \mathrm{C}$ response is dominated by coloumb friction and thus match that of typical inter-ply friction results; therefore, suggesting that resistance to core movement is proportional to the inter-ply friction of the prepreg.

It should be noted however, that with a higher Degree of Cure (DOC) as exhibited in the $180^{\circ} \mathrm{C}$ sample, the contribution of ply resistance to core movement increases. This increase in ply stiffness may, in part, be responsible for the increase in failure pressure between $120^{\circ} \mathrm{C}$ and $180^{\circ} \mathrm{C}$. This is further elaborated in later sections. 


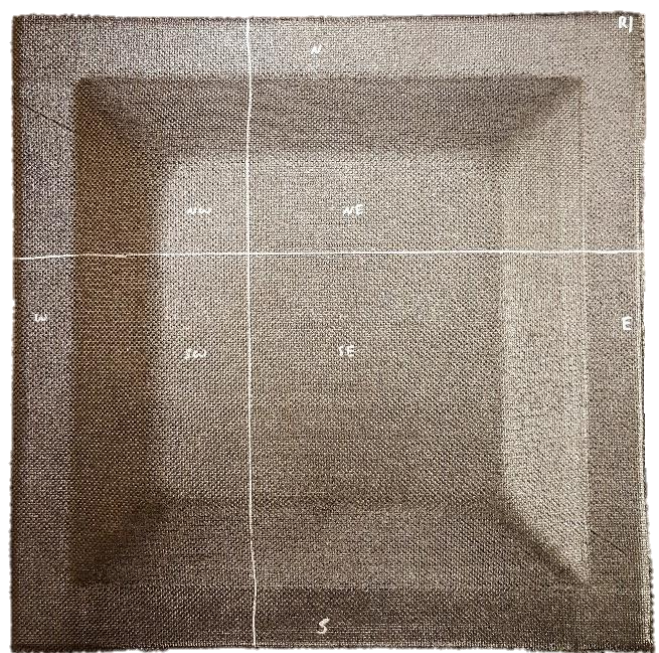

$25^{\circ} \mathrm{C}$

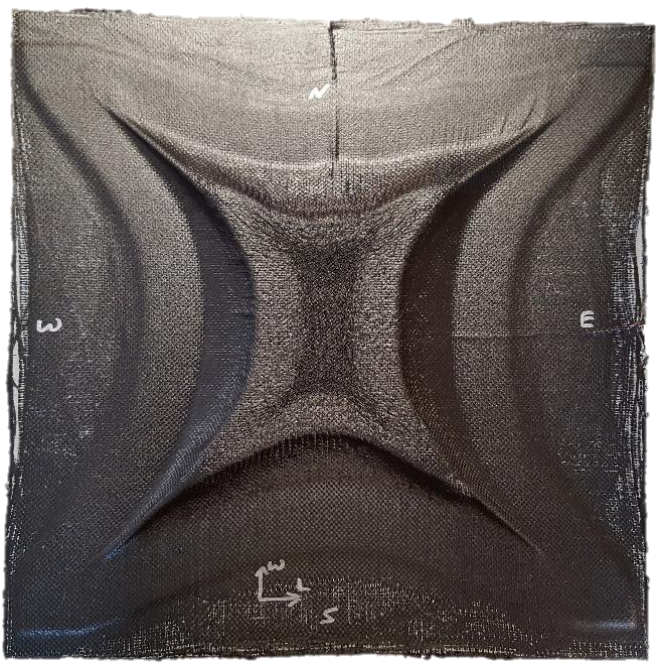

$120^{\circ} \mathrm{C}$

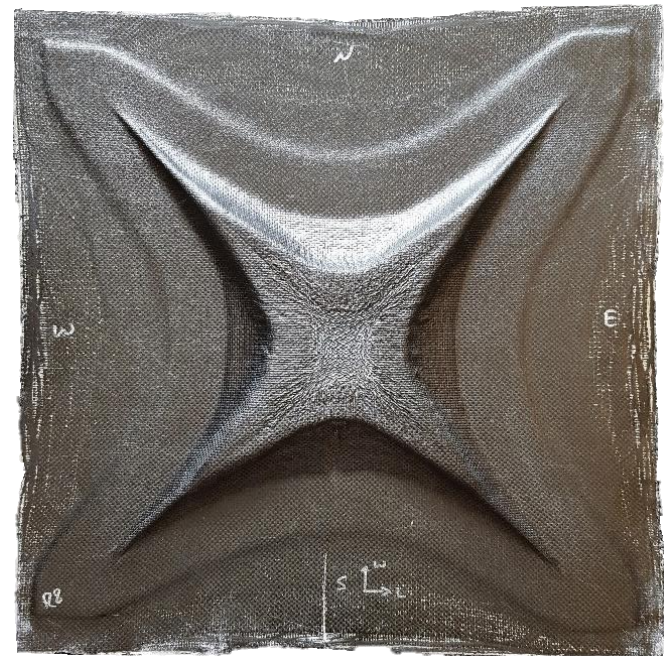

$95^{\circ} \mathrm{C}$

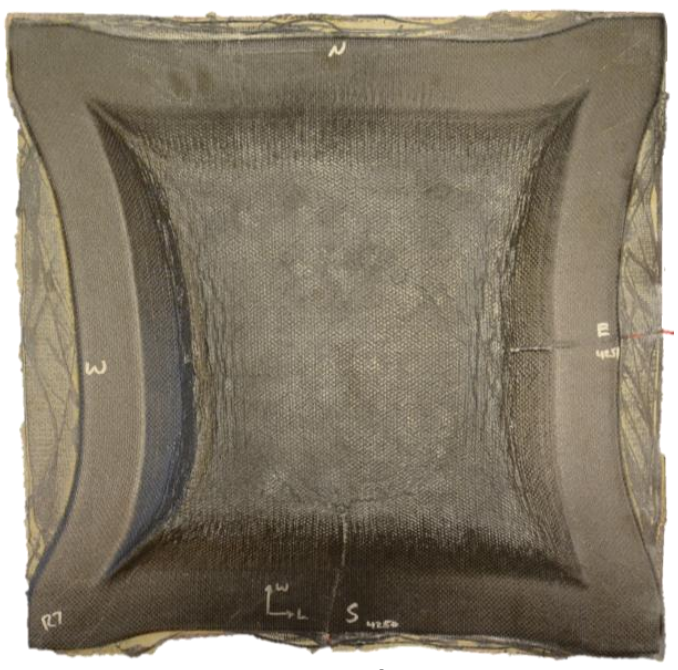

$180^{\circ} \mathrm{C}$

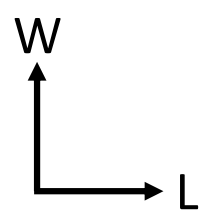

Figure 5.2: $25^{\circ} \mathrm{C}, 95^{\circ} \mathrm{C}, 120^{\circ} \mathrm{C}$, and $180^{\circ} \mathrm{C}$ tests post-processing, showing the extent of core movement.

\section{Extent of core movement}

Although initiation of core movement occurs at similar levels of pressure for the $95^{\circ} \mathrm{C}$ and $180^{\circ} \mathrm{C}$ samples, the extent of core movement varies drastically amongst tests. In the case of the $95^{\circ} \mathrm{C}$ and $120^{\circ} \mathrm{C}$ samples, the extent of core movement is far greater than for the $180^{\circ} \mathrm{C}$ sample as seen in Figure 5.2. This is due to advancement in the $\mathrm{DOC}$ during pressurization. In the case of the $180^{\circ} \mathrm{C}$ test, the panel cures rapidly, effectively solidifying the panel and preventing further movement. Recall that temperature is increased to the set point before pressure is applied. Therefore, for the $180^{\circ} \mathrm{C}$ test, once pressurization occurs, not much time is allowed for crushing to take place before the cure is quite advanced. At onset and collapse the DOC is approximately 0.5 and 0.6 respectively, based on RAVEN simulations. Therefore, gelation has likely already occurred by the time core movement begins for the $180^{\circ} \mathrm{C}$ test. 
The $95^{\circ} \mathrm{C}$ and $120^{\circ} \mathrm{C}$ samples, on the other hand, remain at low viscosity during pressurization and their DOC does not advance significantly. This allows for core movement to continue, rather than halting shortly after initiation as in the $180^{\circ} \mathrm{C}$ case. This is represented by the viscosity profiles given in Figure 4.4. As mentioned previously, the $25^{\circ} \mathrm{C}$ experiment displays no core movement.

\subsubsection{Ply movement}

\section{Gross ply movement}

Surface topography CMM scans of through-thickness cuts as shown in Figure 4.6 allow for thickness changes across the panel surface to be tracked. As core movement progresses, plies are dragged inward to different extents. Changes in thickness across the panel edge gives an indication of the gross movement of the plies. That is, the number of plies at a given location can be inferred from the laminate edge thickness. From this, the extent of ply movement can be deduced. Figure 5.3 provides representative curves of an ideal sample, showing both no core movement and a core movement scenario. In the no core movement scenario (Figure 5.3a), the thickness of the laminate edge remains constant. Moreover, the edgeband encompasses the entirety of the laminate edge as no ply movement occurs. In the core movement case (Figure 5.3b), plies are pulled inward with the core, thus moving the edgeband. Individual plies move to different extents creating a transition in thickness toward the panel edge as plies drop off from the edgeband.

Figure 5.4 shows the thickness changes across the laminate edge of the processed panels in the ribbon and non-ribbon direction for the three samples (CMM scans of the room temperature sample were not performed). A copy of Figure 4.6 is included as a reference of the scanned sections. Near the panel edge some noise is seen which can be disregarded. Measurements of thickness include that of the paint layer, which is not necessarily even among samples. The magnitude of thickness should, therefore, be used as an indicator for the number of plies as opposed to an absolute value.

The edgeband is represented by the relatively flat region at the end of each of the curves. It terminates at the core edge as the thickness begins to rise steeply. In almost every case, excluding the no movement case, the thickness decreases just before the core edge. This indicates a higher level of compaction nearest the core. Alternatively, it could indicate that the core is deforming relative to the plies; however this was not observed when looking at the specific ply movement, as outlined in the next section.

The "no movement" case displays the ideal thickness profile, taken from the full tie-down test. That is, the edgeband extends over the entire laminate edge of the panel with no change in thickness. A minor drop off in thickness is seen nearest the panel edge. This is, however, likely due to plies being slightly offset during layup. Prior to cure, the edgeband represents the entirety of the laminate edge. Following core movement, the plies then move and the edgeband shifts. The $180^{\circ} \mathrm{C}$ sample displays far less ply movement than the other two samples. This is due to the fact that it experienced a lesser degree of core movement as explained in Section 5.1.1. What is of particular interest, however, is the shape of the thickness curves.

Both the $120^{\circ} \mathrm{C}$ and $95^{\circ} \mathrm{C}$ samples display a gradual increase in thickness until the edgeband transi- 

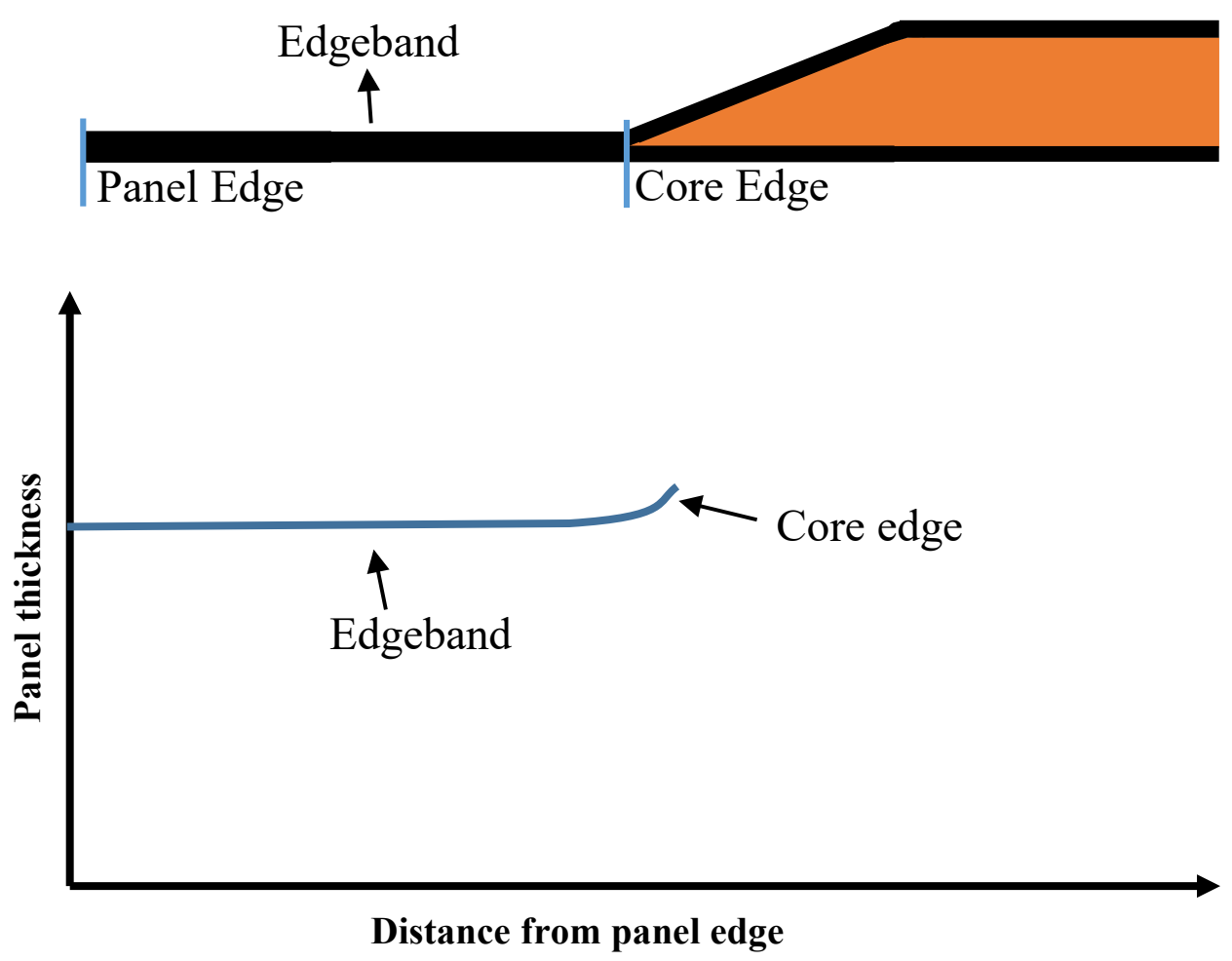

(a) No core movement
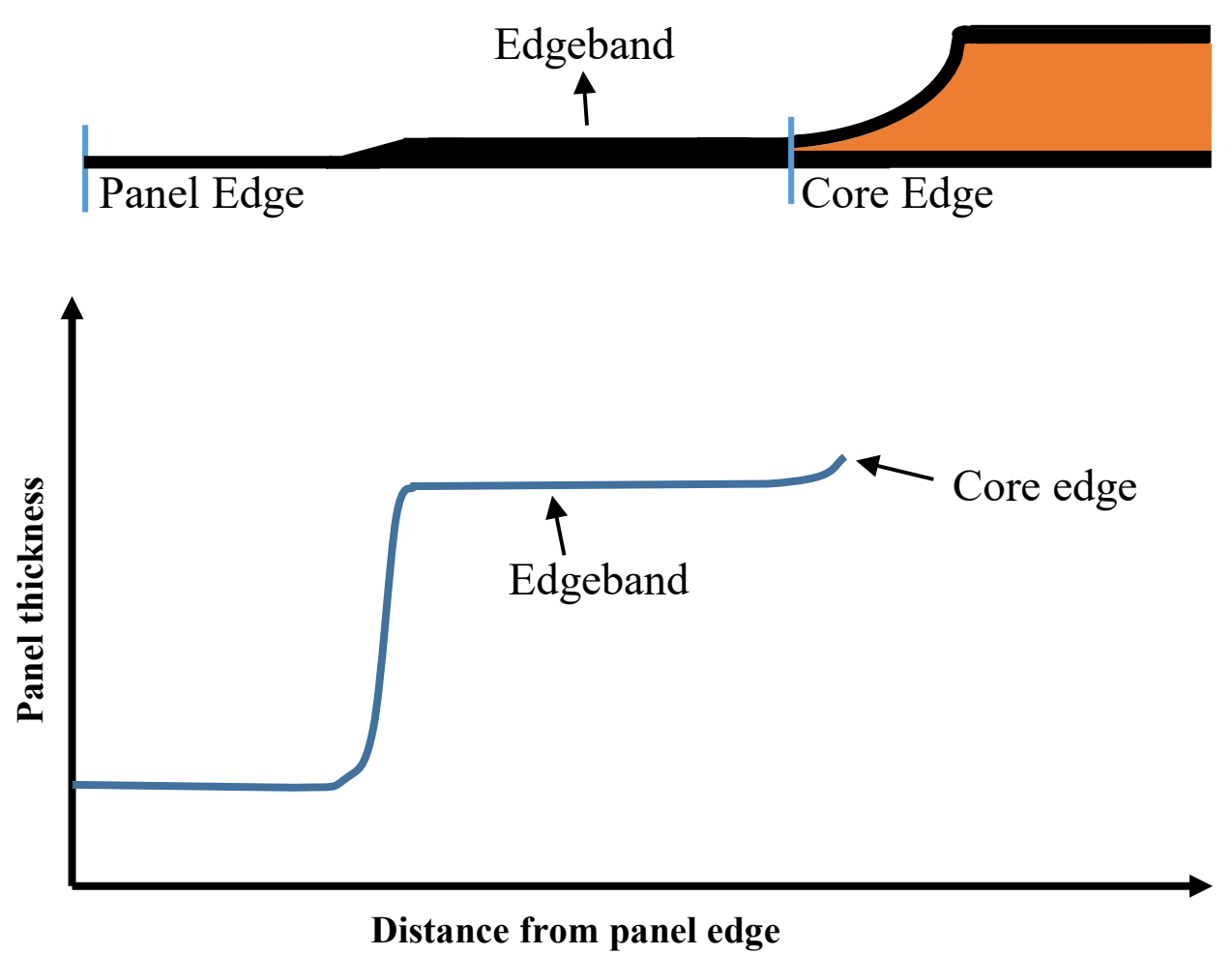

(b) Core movement

Figure 5.3: Schematic showing the expected thickness changes along the laminate edge of a sandwich panel that (a) experiences no core movement and (b) experiences core movement. 


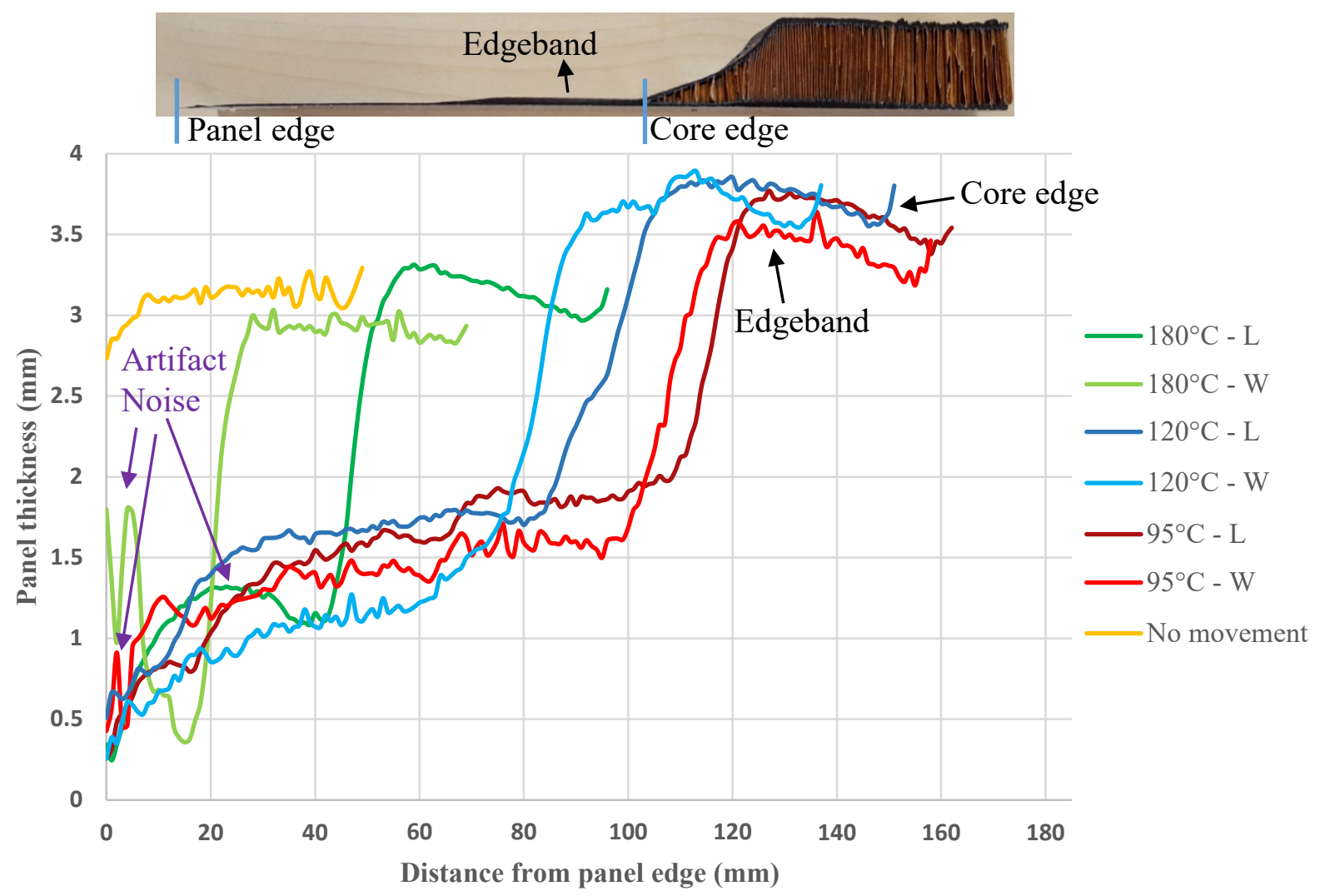

Figure 5.4: Change in thickness across laminate edge of processed panels in the $\mathrm{L}$ and $\mathrm{W}$ directions at varying temperatures. The "No movement" case represents the test with tie down plies, as no visible core movement was seen.

tion, whereas the $180^{\circ} \mathrm{C}$ case transitions rapidly to the edgeband at some distance from the panel edge. This indicates that the lower temperature tests display more relative movement of plies. The gradual change in thickness is the result of plies being offset from one another due to differences in the extent of individual ply movement. The $180^{\circ} \mathrm{C}$ panel shows less relative movement amongst plies, but rather a bulk movement of all plies. This is evident upon visual inspection of the cured panel (Figure 5.2), where the surfacing film is clearly seen up to the edgeband of the $180^{\circ} \mathrm{C}$ sample, indicating all plies have slid on this layer. While the surfacing film is apparent in the other samples, it is not as clear and is still mostly covered by carbon fabric. In fact, surfacing film was seen to seep through interstitial spacings between tows, as shown in Figure 5.5. When few plies are present it can be seen from the surface of the laminate edge.

\section{Specific ply movement}

While Figure 5.4 provides insight into the gross movement of plies, it does not indicate the individual plies that were pulled in with the core. As demonstrated in Figure 4.7, specific ply movement was tracked using digital microscopy. This is shown in Figure 5.6. Again, ply movement is displayed between the panel edge and core edge. 


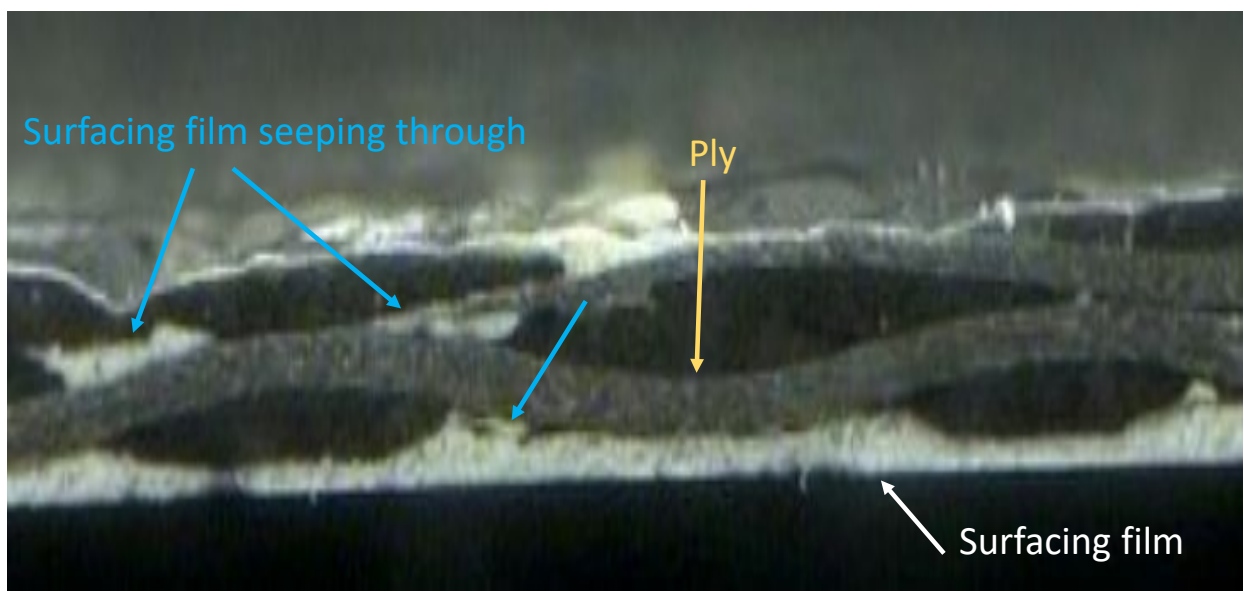

Figure 5.5: Microscope image of laminate edge showing surfacing film seeping through interstitial zones between tows.

In each case the plies nearest the core (recall Figure 4.2) pull in the furthest, as would be expected. However, only in the $180^{\circ} \mathrm{C}$ case does the first ply (i.e. the ply directly against the surfacing film) pull in. It does so in both the ribbon and non-ribbon direction. Moreover, in the $180^{\circ} \mathrm{C}$ sample, the magnitude of individual ply movement does not differ greatly amongst plies. There is notable relative movement between ply 1 and the other plies in the ribbon direction, however this is the only occurance of considerable relative movement for the $180^{\circ} \mathrm{C}$ case. In general, the pattern of movement is such that the plies shift as a unit. This supports the aforementioned notion that bulk motion of plies is favourable at higher temperatures. Similarly, as processing temperature is reduced, relative ply movement increases between plies near the core edge and those further from the core edge. The $95^{\circ} \mathrm{C}$ sample displays considerable relative ply movement on the toolside, while on the bagside inter-ply slip is largely isolated to the ply $11 / 12$ interface. In the case of the $120^{\circ} \mathrm{C}$ sample, the number of toolside interfaces presenting inter-ply slip is less, while on the bagside it is greater. In no case did the surfacing film move with the plies.

In order for core movement to initiate, one bag-side and one-toolside ply must slip. If the plies are treated as rigid bodies, slippage is dictated by the interfaces of least friction. It is impossible for plies further from the core to move without their counterparts nearer the core also moving, as inter-ply shear originates from the core. On the other hand, it is entirely possible that plies near the core could slip while those further away remain stationary. If the weakest interface were between the prepreg and peel ply (bag), then slippage of this layer would result in all bagside plies moving equivalently. Similarly, if the prepreg-surfacing film (tool) interaction represented the weakest toolside interface, then all toolside plies would slip equivalently. Conversely, if a prepreg-prepreg interface exhibited the lowest frictional resistance it would result in relative movement between the plies, where those nearest the core would exhibit the greatest movement. The problem can be thought of as deck of cards where the center of the deck is being pulled. If the top and bottom of the deck are not well supported (i.e. low friction at the tool/bag interface), the whole deck may move. However, if the top and bottom are well supported (i.e. 


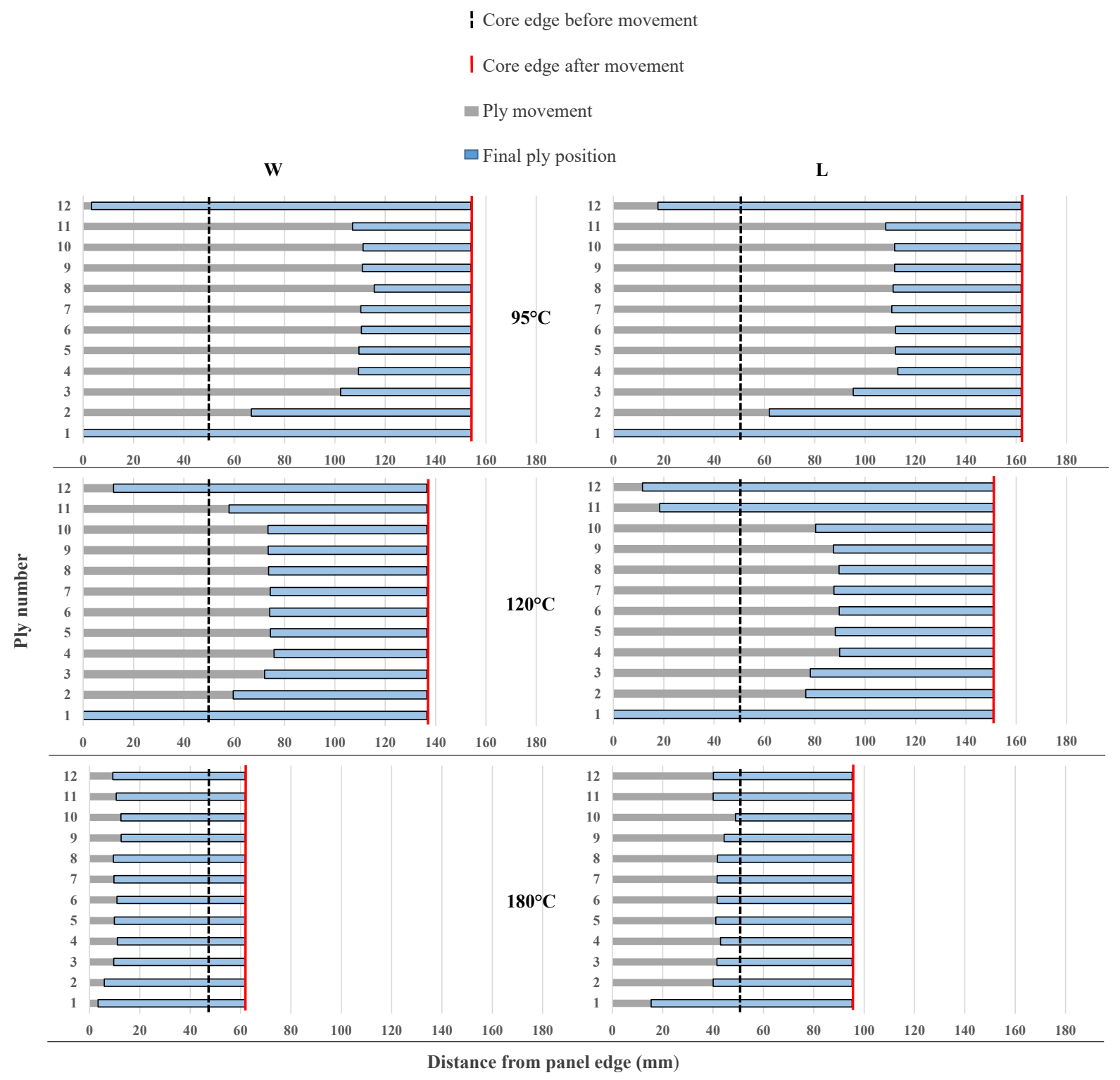

Figure 5.6: Specific ply movement profile of processed panels in $\mathrm{L}$ and $\mathrm{W}$ at varying temperatures. Initially, the core edge is $50.8 \mathrm{~mm}$ from the panel edge.

high friction at the tool/bag interface), a cascading pattern will be observed with cards near the center pulling further than those towards the exterior. The results suggest that high temperature processing promotes the former scenario, whereas low temperature processing results in the latter case.

This trend fits with the previous assertion that the $180^{\circ} \mathrm{C}$ sample exhibited boundary lubrication at the time of core movement, while hydrodynamic friction is still prevalent in the lower temperature samples. In this scenario, coloumb friction dominates the $180^{\circ} \mathrm{C}$ test. Inter-ply friction would therefore increase as a result of fiber intermingling. However, friction between the prepreg-bag and prepreg-tool interface would likely remain unchanged, due to the lack of fiber interactions. It is, therefore, possible that inter-ply friction would increase above that of the prepreg-bag and prepreg-tool interactions. Hence, 
yielding of a prepreg-prepreg interface would not have been the root cause for slip in the $180^{\circ} \mathrm{C}$ case. Rather, the peel ply and surfacing film would have presented the weakest bagside and toolside interfaces, respectively, resulting in all plies sliding relative to these layers. This is opposite to that of the lower temperature samples, where inter-ply friction would remain low due to an inability for fiber interlocking. Another possible explanation for the $180^{\circ} \mathrm{C}$ movement pattern is that because this sample exhibited a higher DOC at the time of pressure application, the resin would have been in a near glassy state. The plies would have been effectively solidified as one mass, demoting the chance for inter-ply movement. At the same time, the friction coefficient between the tool and bagside interface would have decreased as the plies lost their tack, thus promoting slippage across these layers.

The above explanation assumes the plies do experience any strain. It is possible that the extent of ply movement is a combination of pure friction as well tension developed through deformation of the plies. In this case, slippage does not necessarily originate at the interface of least friction but also depends on the number of engaged plies at the interface in question. That is, slip is dictated by the magnitude of friction of the interface as well as the extent of ply tension. If friction is considerably higher than the level of ply tension, then interfacial slip will occur across the interface of least friction regardless. Moreover, At high DOC; , as the plies become stiffer they are likely to slip rather than strain. Therefore, tensile resistance of the plies is only of concern at low DOCs. This explained in more detail later.

It should also be noted that relative motion of plies increases hydrodynamic shear according to Equation 2.15. This is of particular concern for the $95^{\circ} \mathrm{C}$ and $120^{\circ} \mathrm{C}$ samples. It is possible that slippage occurred across a single ply interface, after which shear between neighbouring layers increased, resulting in slippage of further layers. The result would be a cascading pattern of ply movement as seen in Figure 5.6. This may also explain the discrepancy in movement between ply 1 and the other plies in the $180^{\circ} \mathrm{C}$ ribbon direction test. It is possible that, although largely dominated by coloumb friction, hydrodynamic shear still contributed to inter-ply friction in $180^{\circ} \mathrm{C}$ test. In this sense, all plies may have initially slid across the ply 1 interface, after which shear across ply 1 exceeded the frictional limit of the ply 1-surfacing film interface, resulting in slippage across that layer as well.

\section{Relation to mechanical model}

The patterns exhibited in Figure 5.2 and Figure 5.4 indicate that the mechanical nature of ply motion during core movement is influenced by temperature. The interfaces of slip change depending on the processing temperature. Therefore, cure cycle plays a significant role in dictating the manner in which

crush occurs. Although, according to Figure 5.1, this does not necessarily influence resistance to core movement. Nevertheless, current mechanical models do not address this. Regardless of failure pressure, it is important to understand the nature of ply movement as that may dictate core movement mitigation procedures. For example, which plies to tie down.

\subsubsection{Deformation pattern}

Surprisingly, in all three samples the ribbon direction exhibits more deformation than the non-ribbon direction. This is best seen in Figure 5.2 and Figure 5.4, with the magnitude of displacement presented 
Table 5.1: Extent of deformation in the ribbon and non-ribbon direction associated with different processing temperatures.

\begin{tabular}{|c|c|c|}
\hline Test & Displacement in $\mathbf{L ~ ( m m )}$ & Displacement in W $\mathbf{( m m )}$ \\
\hline $95^{\circ} \mathrm{C}$ & 108 & 104 \\
\hline $120^{\circ} \mathrm{C}$ & 100 & 86 \\
\hline $180^{\circ} \mathrm{C}$ & 41 & 16 \\
\hline
\end{tabular}

Table 5.2: Difference in displacement between the ribbon and non-ribbon edges associated with different processing temperatures.

\begin{tabular}{|c|c|}
\hline Test & Difference in displacement between $\mathbf{L}$ and $\mathbf{W}$ edges $(\mathbf{m m})$ \\
\hline $95^{\circ} \mathrm{C}$ & 4 \\
\hline $120^{\circ} \mathrm{C}$ & 14 \\
\hline $180^{\circ} \mathrm{C}$ & 25 \\
\hline
\end{tabular}

in Table 5.1. Moreover, the discrepancy in magnitude is more apparent the higher the processing temperature, with the $95^{\circ} \mathrm{C}$ sample displaying only minor differences in deformation. This is displayed in Table 5.2. The values provided are measured from a single edge. The total discrepancy in ribbon versus non-ribbon deformation would, therefore, be twice the amount shown, assuming the deformation pattern is symmetric.

The section cuts used to obtain the individual ply movement profiles were not always the exact center of the panel. Extent of crush was, however, directly measured along the center line of the panels. As a result, the extent of core movement displayed in Figure 5.6 may not align exactly with that provided in Table 5.1

Interestingly, in-situ data indicates onset of failure occurs in both directions simultaneously. Although, at higher processing temperatures, crush in the ribbon direction progresses at a notably faster rate following collapse. This is shown in Figure 5.7. For the $95^{\circ} \mathrm{C}$ sample, the two LVDT; measure a similar rate of deformation. However, for the $180^{\circ} \mathrm{C}$ sample, the LVDT in the L direction deforms at a quicker rate than its counterpart in the $\mathrm{W}$ direction. Unfortunately, data on the rate of progression of core movement in the $\mathrm{L}$ and $\mathrm{W}$ directions is not available for the $120^{\circ} \mathrm{C}$ sample, since LVDTs were placed on equivalent sides of the panel for this test. However, a similar pattern to the $180^{\circ} \mathrm{C}$ sample was observed in the $120^{\circ} \mathrm{C}_{\text {orig }}$ experiment (refer to Section 4.5.2 for a description of the test). The results of this test are provided in Appendix C. It should be noted that the LVDTs do not necessarily capture the full extent of core movement as they are limited by their stroke length. Hence, when the LVDTs indicate no further movement is occurring, this may not actually be the case.

The difference in magnitude between crush in the ribbon versus non-ribbon direction may be attributed to the manner in which cell collapse occurs in the two directions. Recall that under uniaxial loading in the $\mathrm{L}$ direction, NOMEX ${ }^{\circledR}$ honeycomb displays homogenous collapse of the cellular structure by buckling of the cell walls along the load path. Conversely, loading in the $\mathrm{W}$ direction results in a row-by-row collapse pattern. The homogenous buckling of cells in the L direction may present itself as a quicker or preferential mode of deformation once collapse initiates. Hence, for high temperature 


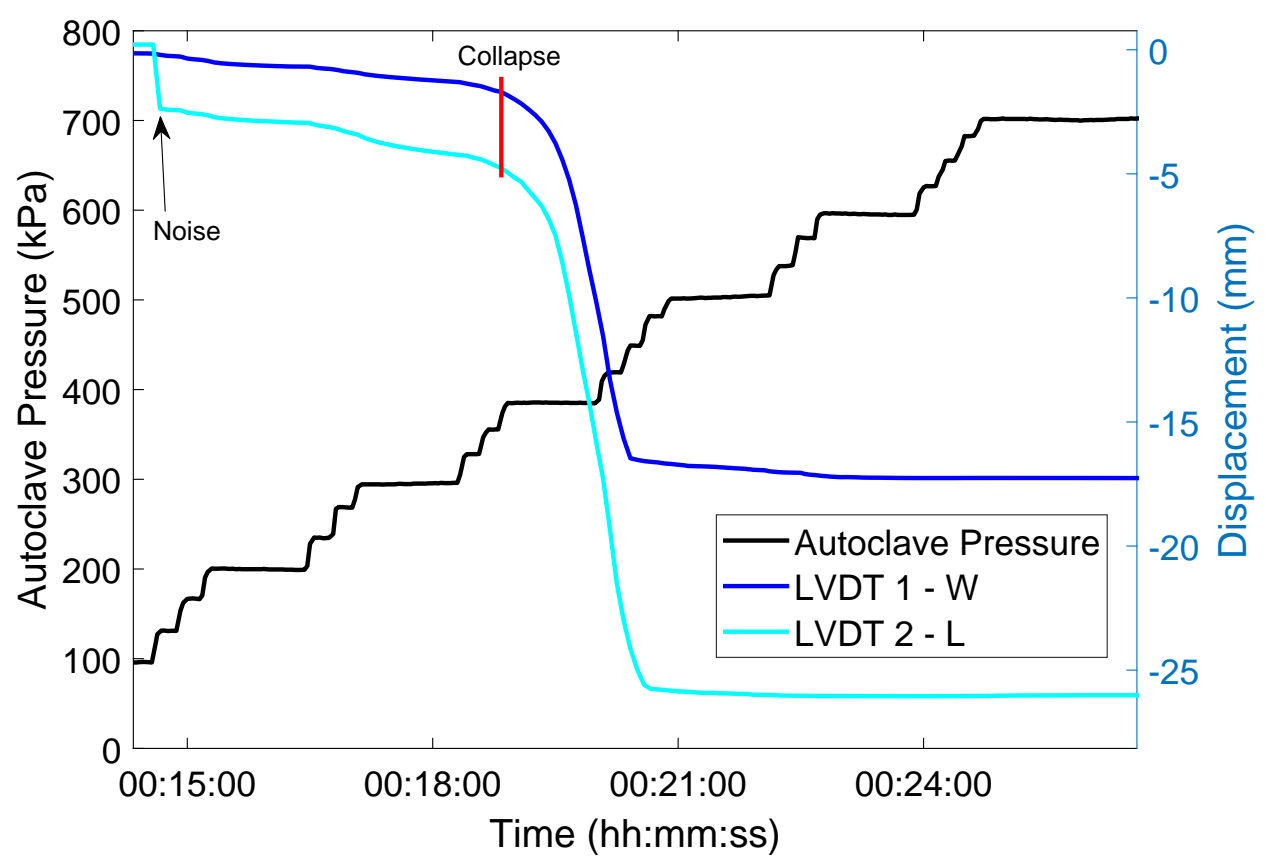

(a) $95^{\circ} \mathrm{C}$

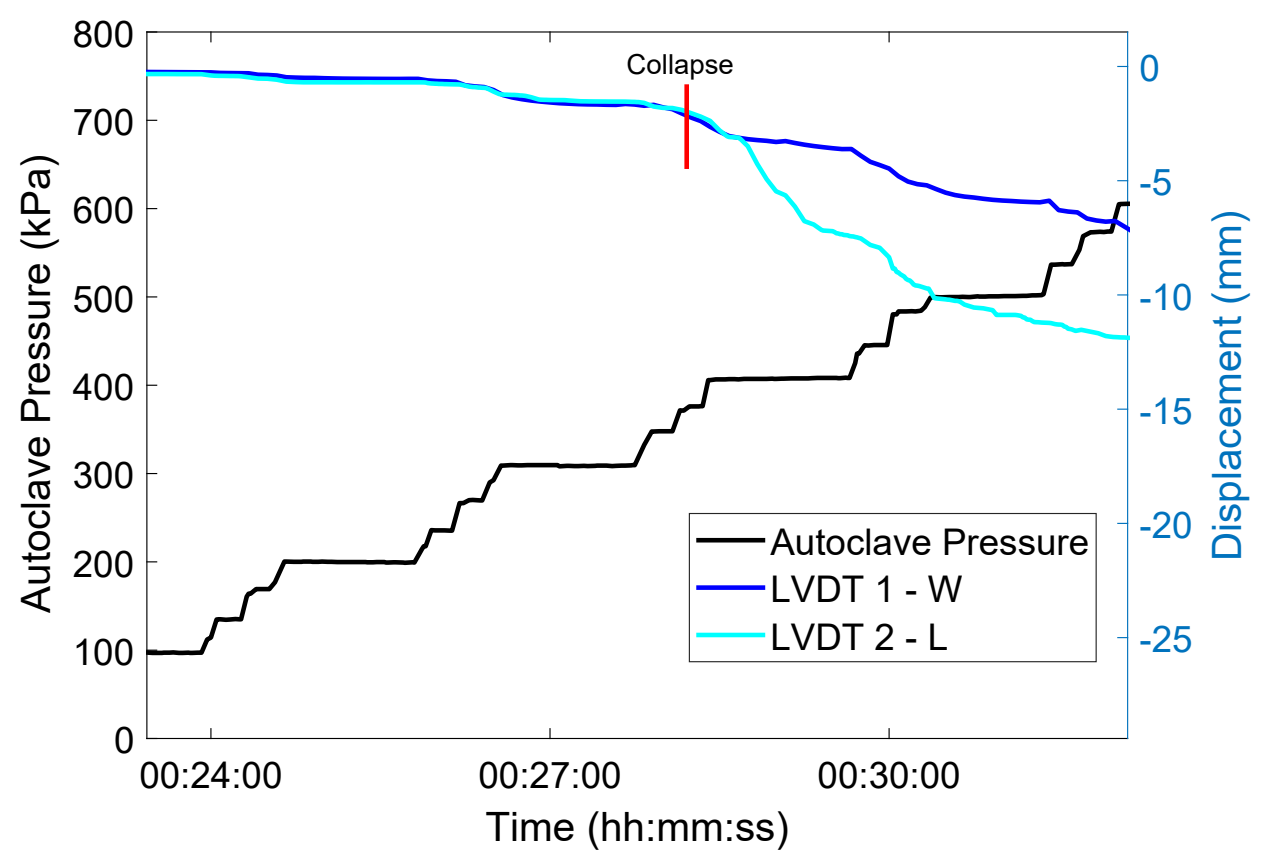

(b) $180^{\circ} \mathrm{C}$

Figure 5.7: Core movement in ribbon and non-ribbon directions for (a) low versus (b) high temperature processing. Autoclave pressure is absolute external pressure. Vacuum pressure is not shown. 
processing, where the period of core movement is short due to the rapidly advancing DOC, deformation in the $\mathrm{W}$ direction does not have time to progress significantly. Conversely, for low temperature processing, the extended core movement window allows for the slower $\mathrm{W}$ deformation to catch up in magnitude to the deformation exhibited in the L direction. 


\subsection{Effect of pressure}

It is beneficial to understand the range of pressure necessary to cause core movement at a given temperature. However, this begs the question, for a given pressure at what temperature does core movement occur? This is more indicative of a "real-world" processing scenario, as often pressure is set and temperature is then increased according to a specific cure cycle. The following section aims to answer this question. Tests conducted include those listed under "Effect of Pressure" in Table 4.1.

\subsubsection{Failure temperature}

The two experiments conducted are representative of typical processing pressures seen in processing of laminates and sandwich panels. The former pressure being $600 \mathrm{kPa}$ gauge and the latter $325 \mathrm{kPa}$ gauge. Recall that net pressure is the absolute external pressure minus the pressure within the vacuum bag. Failure of the panels occurred after venting of the vacuum bag (i.e. pressure within the bag was $1 \mathrm{~atm}$ ), therefore the net pressure acting on the panels is also 600 and $325 \mathrm{kPa}$. Onset of the $600 \mathrm{kPa}$ sample, however, occurred while pressure was still increasing due to pressure-induced heating. That said, for simplicity, the two samples are referred to by their set processing pressures, 325 and $600 \mathrm{kPa}$.

The temperature that the two samples failed at is displayed in Figure 5.8a. For the $325 \mathrm{kPa}$ sample, onset and collapse occurred at $54.6^{\circ} \mathrm{C}$ and $82^{\circ} \mathrm{C}$ respectively. The $600 \mathrm{kPa}$ sample showed slightly different results as onset occurred before maximum pressure was achieved. As pressure increases, temperature also increases according to the ideal gas law. In this case, the temperature reached a critical point sufficient to result in the onset of core movement. This occurred at a net pressure and temperature of $497 \mathrm{kPa}$ and $34.2^{\circ} \mathrm{C}$. Collapse occurred later after the set pressure $(600 \mathrm{kPa})$ was achieved and convection heating began. This occurred at a temperature of $42.6^{\circ} \mathrm{C}$. The fact that onset occurred near room temperature and without any external heating is testament to the necessity for strict processing conditions in order to prevent core movement. The room temperature sample presented in the previous section displayed no core movement, yet underwent a processing cycle that was, initially, very similar to that of $600 \mathrm{kPa}$ sample. That is, pressure was rapidly increased to $600 \mathrm{kPa}$ with no external heating applied during this step. In the room temperature sample however, the maximum temperature within the autoclave was only $30^{\circ} \mathrm{C}$ due to pressurization and remained for a relatively short period of time approximately two minutes. The $600 \mathrm{kPa}$ sample presented here, had been held above $30^{\circ} \mathrm{C}$ for around four minutes before onset was observed. This further demonstrates the stringent tolerances involved in core movement, as an apparent $4^{\circ} \mathrm{C}$ rise in temperature held over a longer time period can be the difference as to whether or not core movement initiates. Moreover, the room temperature sample had actually reached a higher pressure $(600 \mathrm{kPa})$ without core movement initiating. Here, however, the sample displayed onset at a lower pressure $(497 \mathrm{kPa})$ due to a slight increase in temperature. This stresses the importance of hydrodynamic friction in resisting core movement during the early stages of processing. If the resin viscosity remains high enough during the hydrodynamic regime, then core movement will not initiate for pressures up to $600 \mathrm{kPa}$. However, slight increases in temperature will decrease the resin viscosity which may tip the scales in favour of core movement as shown here.

Figure $5.8 \mathrm{~b}$ shows the resin viscosity at failure for the two samples. The values presented are 


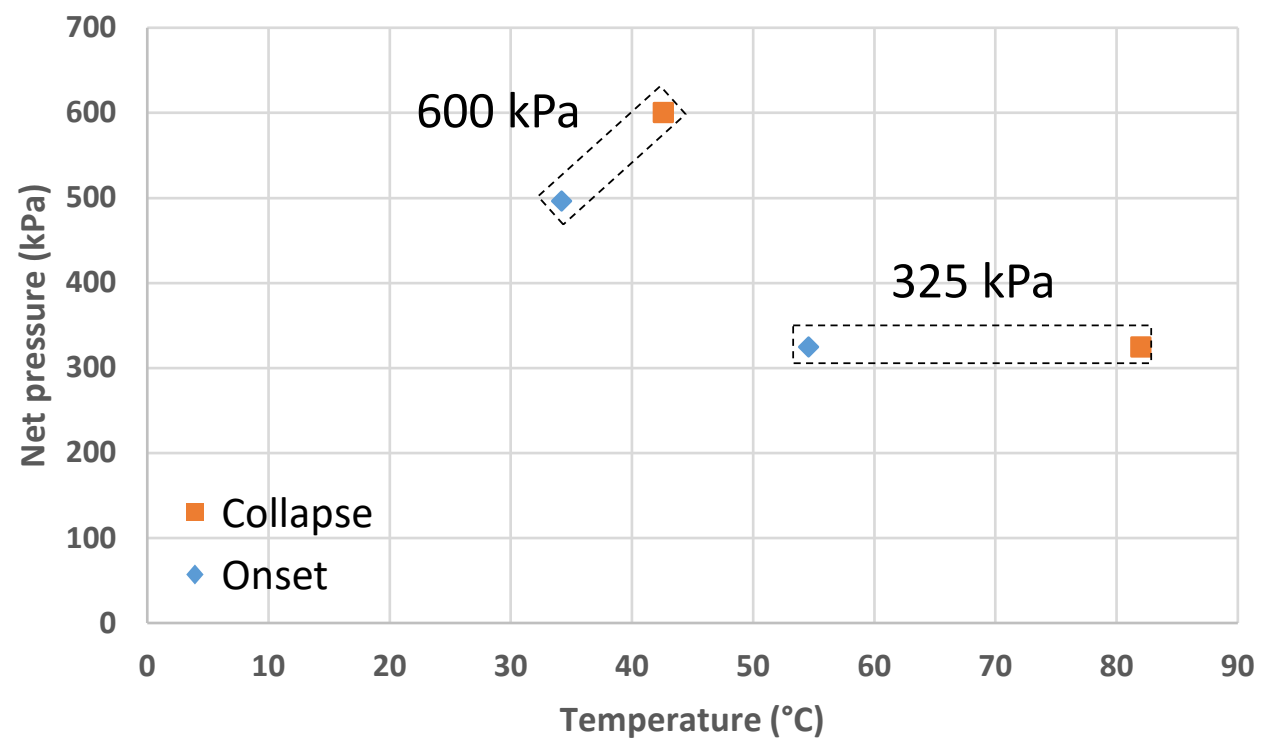

(a) Temperature at failure for various processing pressures

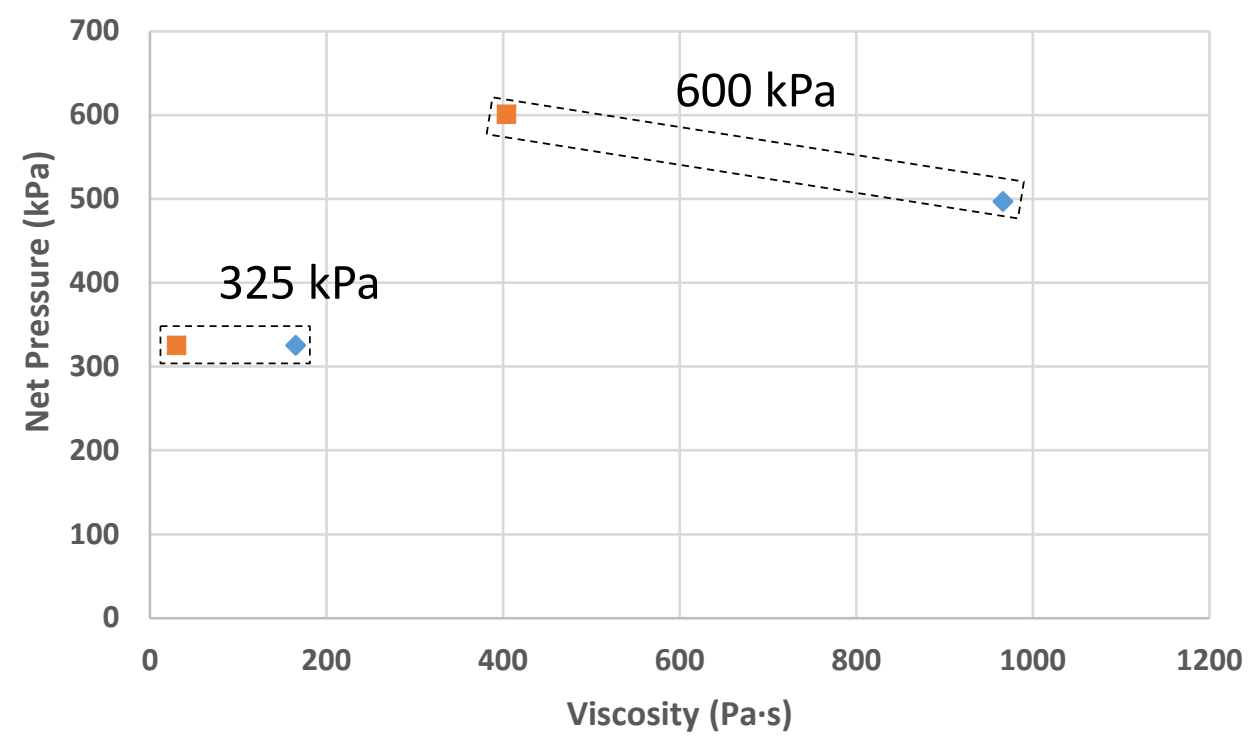

(b) Resin viscosity at failure

Figure 5.8: (a) Temperature at which onset and collapse occur for sandwich panels processed at a specific pressure and (b) the associated resin viscosity at failure. 
estimates based off RAVEN viscosity curves as demonstrated in Figure 4.4. The initial pressure-induced heating occurs at an approximate rate of $3^{\circ} \mathrm{C}$ per minute, whereas the RAVEN viscosity curves are based off a $5^{\circ} \mathrm{C}$ per minute heating cycle. Further, as the pressure approaches the set value, the rate of pressurization slows, thus decreasing the rate of pressure-induced heating. As such, the viscosity values are approximates. In this set of experiments viscosity decreases between onset and collapse. This is because onset and collapse occur during the heatup stage, where gelation has not yet occurred. Therefore, as temperature is increased, viscosity decreases. It is likely the friction regime remains hydrodynamic throughout the core movement process for both samples.

The resin viscosity at onset and collapse for the $325 \mathrm{kPa}$ sample is approximately 165 and $30 \mathrm{~Pa} \cdot \mathrm{s}$ respectively. Viscosity at onset and collapse for the $600 \mathrm{kPa}$ sample is much higher, approximately 966 and $404 \mathrm{~Pa} \cdot \mathrm{s}$. This is in line with the expected response. At higher pressure, the temperature, and associated resin viscosity, necessary to allow for core movement is reduced. Interestingly, the temperature to cause core movement decreases linearly with an increase in pressure. Onset at $325 \mathrm{kPa}$ occurs at a temperature of $55^{\circ} \mathrm{C}$ and onset at $497 \mathrm{kPa}$ occurs at a temperature of $34^{\circ} \mathrm{C}$. Similarly, collapse at $325 \mathrm{kPa}$ occurs at a temperature of $82^{\circ} \mathrm{C}$ and collapse at $600 \mathrm{kPa}$ occurs at a temperature of $43^{\circ} \mathrm{C}$. In both scenarios the temperature at failure is inversely proportional to the increase in pressure. As evidenced through the room temperature sample, however, there is a minimum temperature requirement to cause core movement for the range of pressures discussed. Following the above relation, at a pressure of $600 \mathrm{kPa}$, one would assume core movement should initiate above a temperature of $29^{\circ} \mathrm{C}$. That was not observed to be the case for the room temperature sample though, where net pressure was $600 \mathrm{kPa}$ and the autoclave temperature reached $30^{\circ} \mathrm{C}$.

In these set of experiments, the critical zone is no longer a range of pressures but rather a range of temperatures. At a processing pressure of $325 \mathrm{kPa}$ the critical zone is $27.4^{\circ} \mathrm{C}$, whereas at a processing pressure of $600 \mathrm{kPa}$ the critical zone is reduced to $8.4^{\circ} \mathrm{C}$. Since onset in the latter case actually occurred at a pressure around $500 \mathrm{kPa}$, it is expected that the true critical zone at $600 \mathrm{kPa}$ is even less, assuming the minimum temperature requirement is satisfied. Therefore, at high pressures, temperature fluctuations within the autoclave may bear significant consequences with regards to core movement.

\subsubsection{Ply movement}

\section{Gross ply movement}

Just as in Section 5.1 panel thickness across the laminate edge was measured in order to gain an understanding of the scale of ply movement. The results are displayed in Figure 5.9. Recall Figure 5.3 when interpreting the figure. Note also that the magnitude of thickness includes that of the paint layer. As a result, some error is introduced.

The pattern of movement among the two samples is fairly similar with the major difference lying in the extent of movement. At higher pressure, core movement is more severe as expected. In both cases, there is a gradual increase in panel thickness until the edgeband. This indicates that in the two experiments, plies are sliding relative to one another resulting in plies dropping off at different locations 


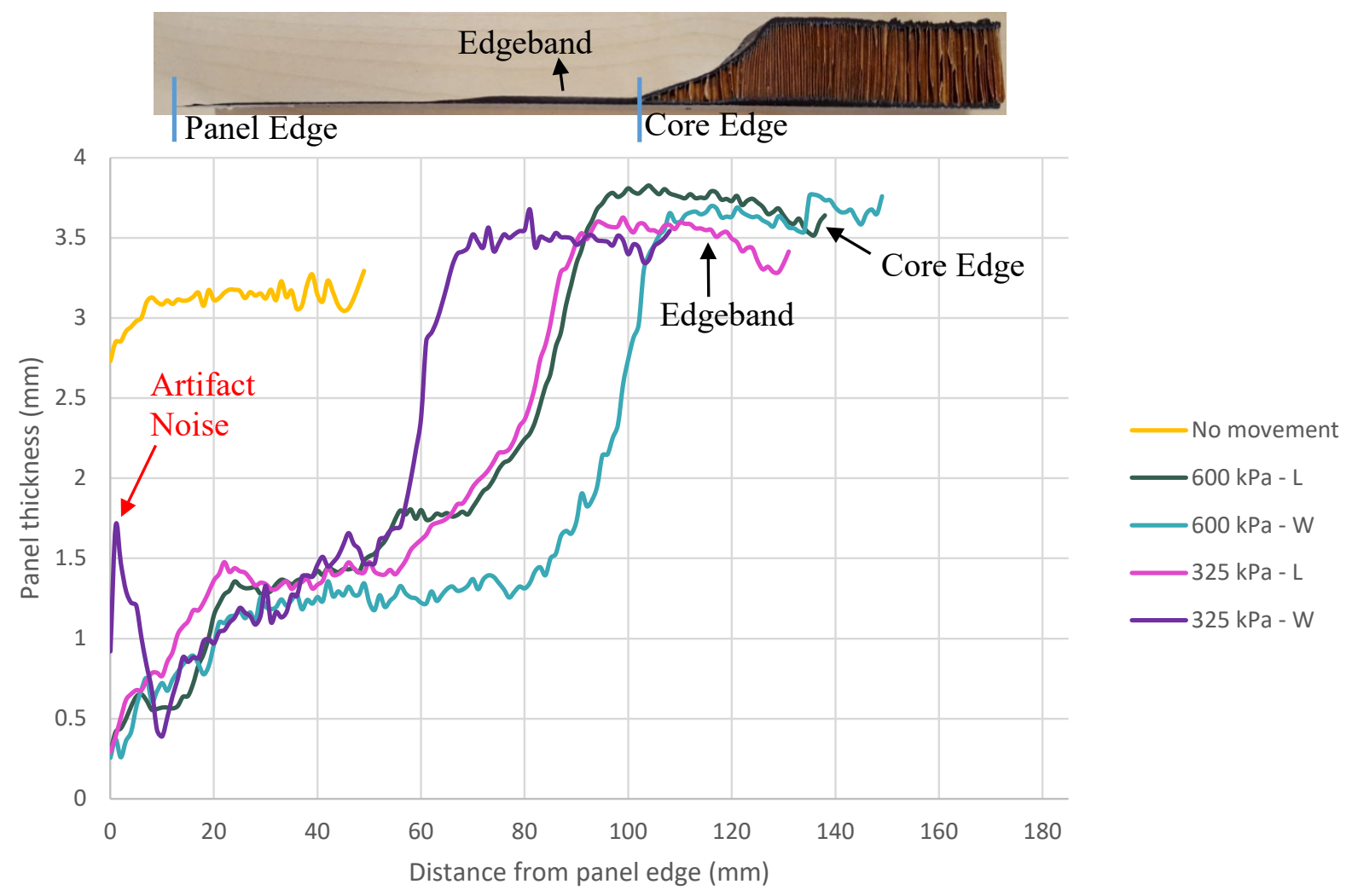

Figure 5.9: Change in thickness across laminate edge of processed panels in the $\mathrm{L}$ and $\mathrm{W}$ directions at varying pressures. The "No movement" case represents the test with tie down plies, as no visible core movement was seen.

along the laminate edge. In the previous section, low temperature processing showed similar results. Since movement occurs during the heating phase, resin has likely not infiltrated the core of the tows, meaning a considerable amount of surface resin is present. As a result, the friction mode would be hydrodynamic in nature. Hence, the frictional resistance to crush would be dominated by properties of the resin. Ply drop offs are, therefore, expected. The shear force between plies increases as neighbouring plies slip, according to Equation 2.15. Once plies nearest the core begin to slip, additional plies would follow thereafter, resulting in a cascading pattern.

\section{Specific ply movement}

The individual movement of each ply is presented in Figure 5.10. For the most part, the pattern of movement among the high and low pressure samples are similar; with only the extent of movement differing. Moreover, considerable relative ply movement is seen which matches that of a hydrodynamic friction regime. For the $600 \mathrm{kPa}$ sample, ply 1 moves slightly in the $\mathrm{L}$ and $\mathrm{W}$ directions. The only other occurrence of this happening is in the $180^{\circ} \mathrm{C}$ sample. Unlike in the $180^{\circ} \mathrm{C}$ test, however, the plies do not move as a bulk. It is therefore likely that with a predominant hydrodynamic friction regime and a relatively high viscosity at failure (Figure 5.8(b)), the hydrodynamic shear stress acting on ply 1 was 


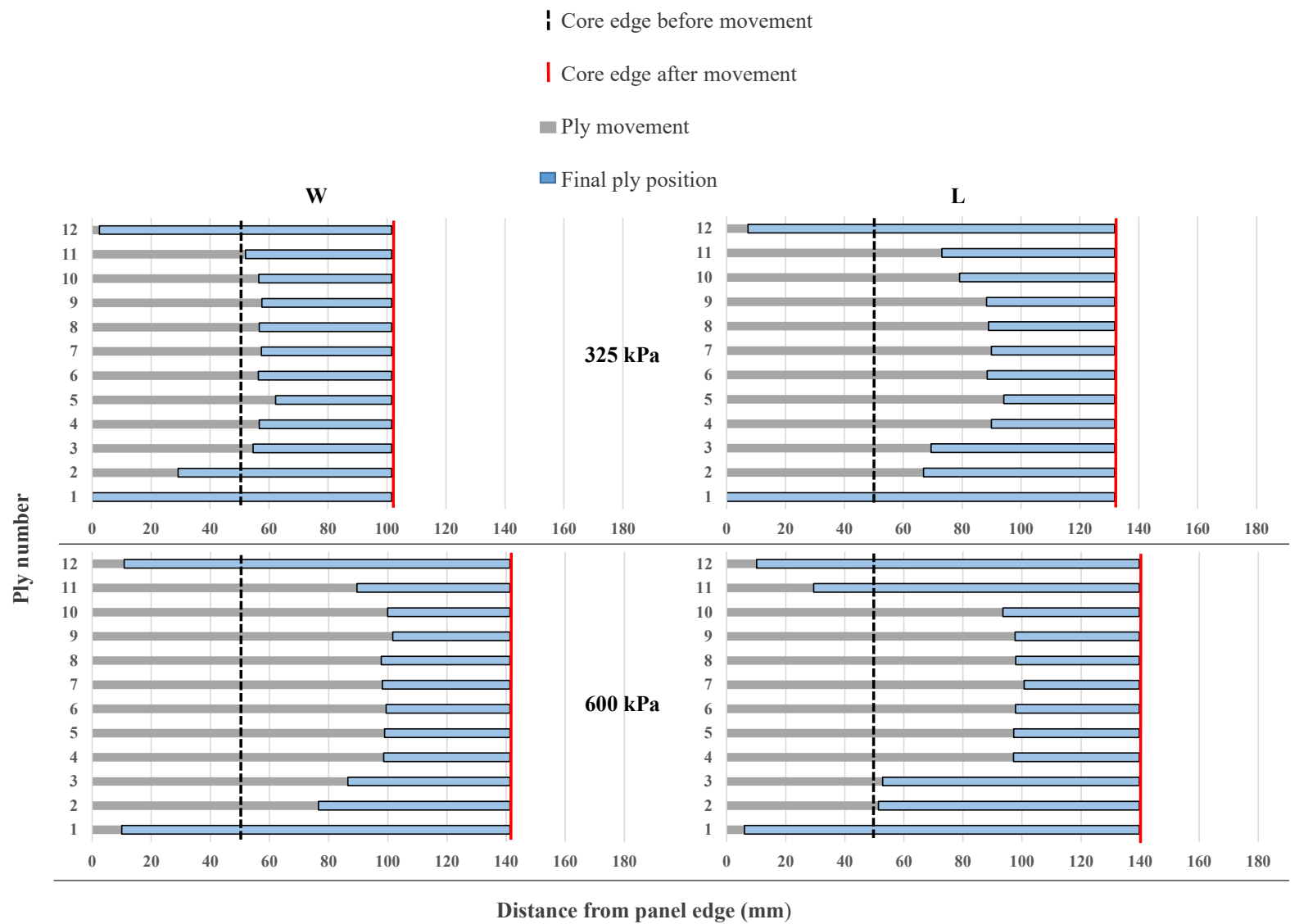

Figure 5.10: Specific ply movement profile of processed panels in $\mathrm{L}$ and $\mathrm{W}$ at varying pressures. Initially, the core edge is $50.8 \mathrm{~mm}$ from the panel edge.

sufficient to result in slippage across the ply 1-surfacing film interface. The same phenomenon is not observed in the lower pressure experiment; likely due to the decrease in applied pressure and subsequent reduction in resin viscosity at failure. While the measured deformation of ply 1 in the L direction sample for the $600 \mathrm{kPa}$ test was around $6 \mathrm{~mm}$, the opposing L edge showed an approximate deformation of 16 $\mathrm{mm}$ for ply 1 . The two $\mathrm{W}$ edges however, showed similar levels of displacement for ply 1 . Generally speaking the deformation pattern is fairly symmetric amongst equivalent edges, however some variance is occasionally seen.

In a sense, because the friction mode does not change throughout the time period over which core movement occurs, managing core movement becomes simpler. Higher processing pressures result in a reduction of temperature necessary for core movement initiation. The mechanical progression of core movement does not change however. Since most cure cycles involve rapid pressurization coupled with slower heating, this represents the general relation for most situations in which core movement would occur. Although high temperature processing affects the mechanical nature of core movement (presented in the previous section), if heating is simultaneously performed with pressurization, then core movement is likely to occur before the processing temperature is achieved. Thus, the effects of high temperature processing can be negated in this scenario. 


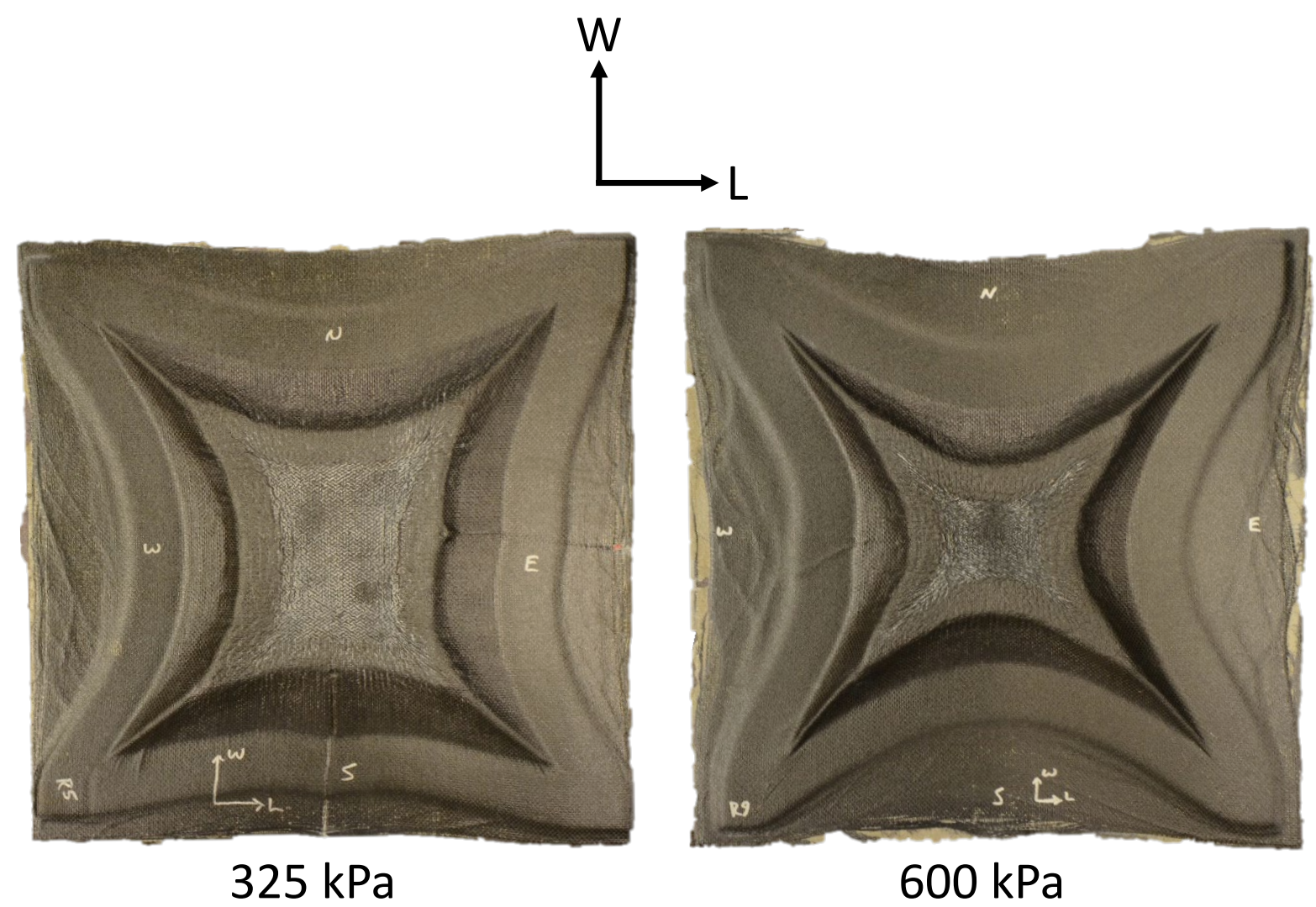

Figure 5.11: 325 and $600 \mathrm{kPa}$ tests post-processing, showing the extent of core movement.

\subsubsection{Extent of core movement}

As before, deformation in the ribbon direction is more extensive than in the non-ribbon direction for the $325 \mathrm{kPa}$ sample. However, for the $600 \mathrm{kPa}$ sample, deformation in the non-ribbon direction is slightly greater than in the ribbon direction. This is the only instance of this occurring among the experiments conducted. Images of the two samples, post-processing, are shown in Figure 5.11 with their respective magnitudes of displacement presented in Table 5.3. Recall that extent of crush is measured directly along the center line of the panels and do not necessarily match the core position presented in Figure 5.10.

Near a given edge, the collapse pattern is largely uniaxial in nature. That is, cells fail according to the ribbon or non-ribbon collapse mode (i.e. buckling versus plastic bending). However, towards the center of the panel, as adjacent edges are pushed together, the collapse pattern takes on a biaxial state. A combination of buckling and plastic bending is seen. It is possible that the reason deformation in the $\mathrm{L}$ direction is higher for all samples except one, is that homogeneous, buckling is a favourable failure mode and therefore will usually dominate the biaxial response. However, as suggested by the $600 \mathrm{kPa}$ sample, plastic bending may "win" the response on occasion, allowing for higher deformation in the W direction. This is further shown in Section 5.5.2.

Interestingly, despite showing greater overall displacement in the $\mathrm{W}$ direction, the $600 \mathrm{kPa}$ sample exhibited a slightly faster rate of displacement in the L direction initially. This is shown in Figure 5.12. 
Table 5.3: Extent of deformation in the ribbon and non-ribbon direction associated with different processing pressures.

\begin{tabular}{|c|c|c|}
\hline Test & Displacement in L (mm) & Displacement in W (mm) \\
\hline $325 \mathrm{kPa}$ & 78 & 52 \\
\hline $600 \mathrm{kPa}$ & 85 & 94 \\
\hline
\end{tabular}

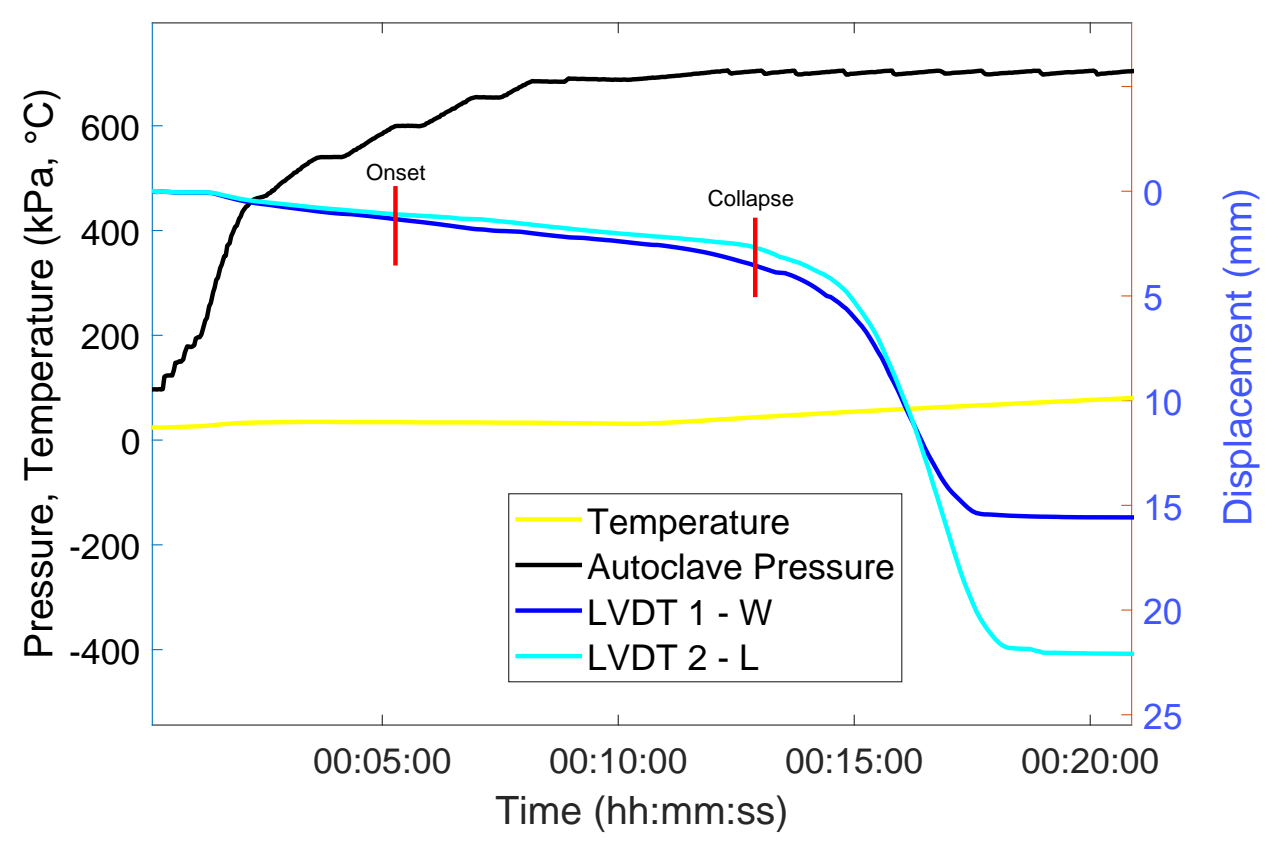

Figure 5.12: Core movement in ribbon and non-ribbon direction for high pressure (600 kPa) processing. Autoclave pressure is absolute external pressure. Vacuum pressure is not shown.

Recall that the LVDT; only capture the initial stages of core movement before their stroke lengths are reached. This indicates that the initial rate of deformation does not necessarily correspond to the final magnitude of deformation. Rather, the rate of progression of the crush front is important as the crush zone that projects further into the core quicker, will likely dominate the failure mode. In this case, the W collapse front "won" the biaxial response. This is explained in Section 5.5.2. Unfortunately, rate of progression of the two edges is not available for the $325 \mathrm{kPa}$ sample, as one of the LVDTs failed. 


\subsection{Effect of layup}

The following section details the effect various layup features have on core movement. This includes both the initiation of core movement as well as the manner in which it progresses. Tests conducted include those listed under "Effect of Layup" in Table 4.1.

\subsubsection{Failure pressure}

Similar to Section 5.1.1 the net pressure at onset and collapse was determined for each of the experiments conducted. The results are presented in Figure 5.14. Unlike the previous tests, viscosity does not vary among samples since all tests were conducted at the same processing temperature of $120^{\circ} \mathrm{C}$. The full tie down test displayed no notable core movement and so is not presented in Figure 5.14. That said, minor distortions in the cellular pattern were seen upon sectioning the panel (Figure 5.13). However, these were not externally visible and likely were not picked up by the LVDTs.

Not surprisingly, restraining only two edges (i.e. half tie downs) of the plies allows for movement along the unrestrained edges while, at the same time, restricting movement along the restrained edges. Interestingly, the resistance to movement increases along the unrestrained edges in comparison with the standard layup, indicating that core movement across adjacent edges are somewhat coupled. The critical zone also increases in size, providing a larger buffer between onset and collapse. Onset and collapse occur at 183 and $252 \mathrm{kPa}$ respectively for half tie downs, as opposed to 170 and $200 \mathrm{kPa}$ for the standard layup.

CMS slightly increases the resistance to core movement, with onset and collapse occurring at 189 and $225 \mathrm{kPa}$ respectively. The critical zone remains similar in size however. It was observed from video footage that crush initiates beyond the stabilized zone which extends past the chamfer radius. For all other samples crush initiates around the radius of the core. CMS therefore, prevents crush from occurring along the stabilized zone while providing resistance to crush in the unstabilized zone. This is further detailed in Section 5.5.2.

As would be expected, a steeper chamfer angle reduces the force required for core movement with onset and collapse occurring at 152 and $183 \mathrm{kPa}$ respectively. Again, the size of critical zone does not change however. Interestingly, in the $45^{\circ}$ core test, collapse occurs immediately following venting of

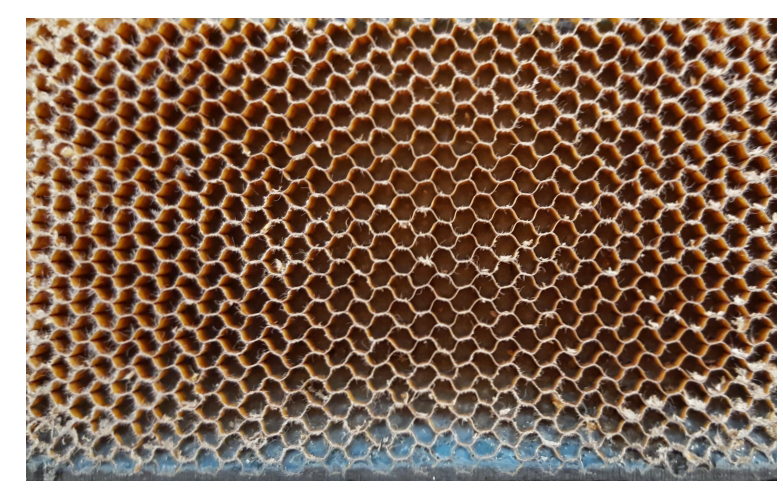

Figure 5.13: Minor deformations in cellular pattern of full tie down test. 


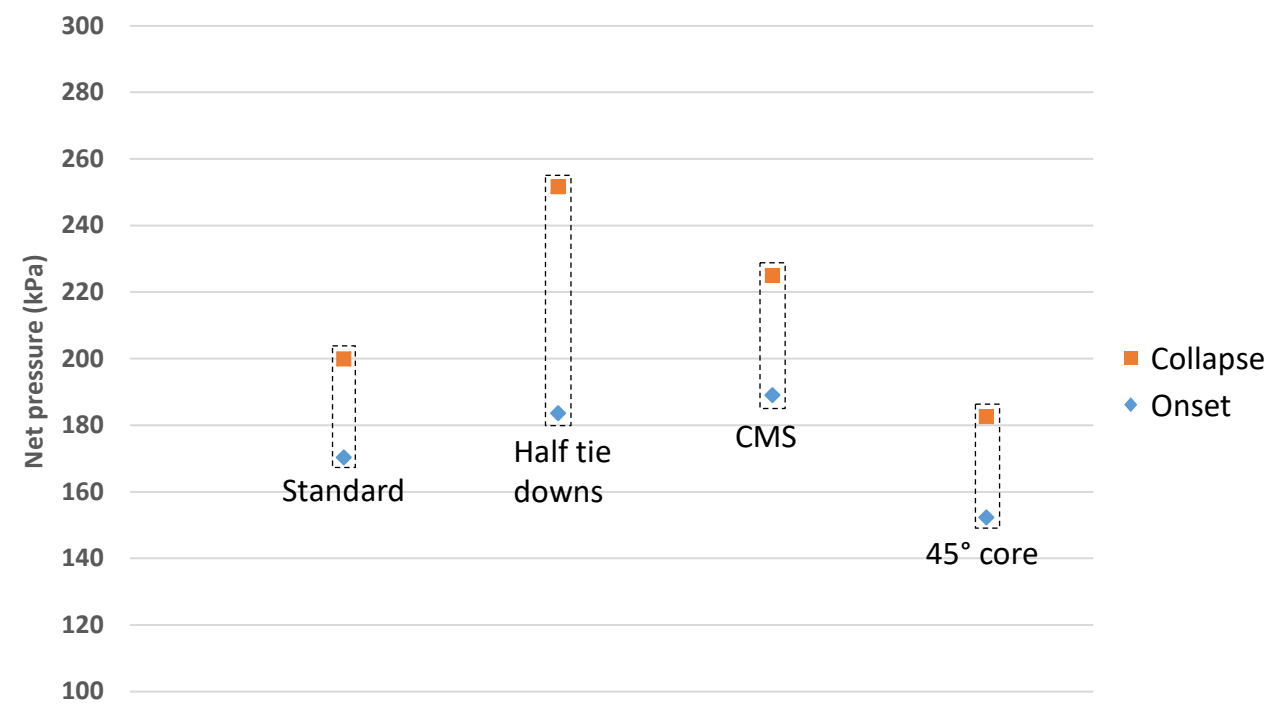

Figure 5.14: Net pressure acting on the sandwich panels at onset and collapse for varying layups. The data for half tie downs represents that of the unrestrained edges.

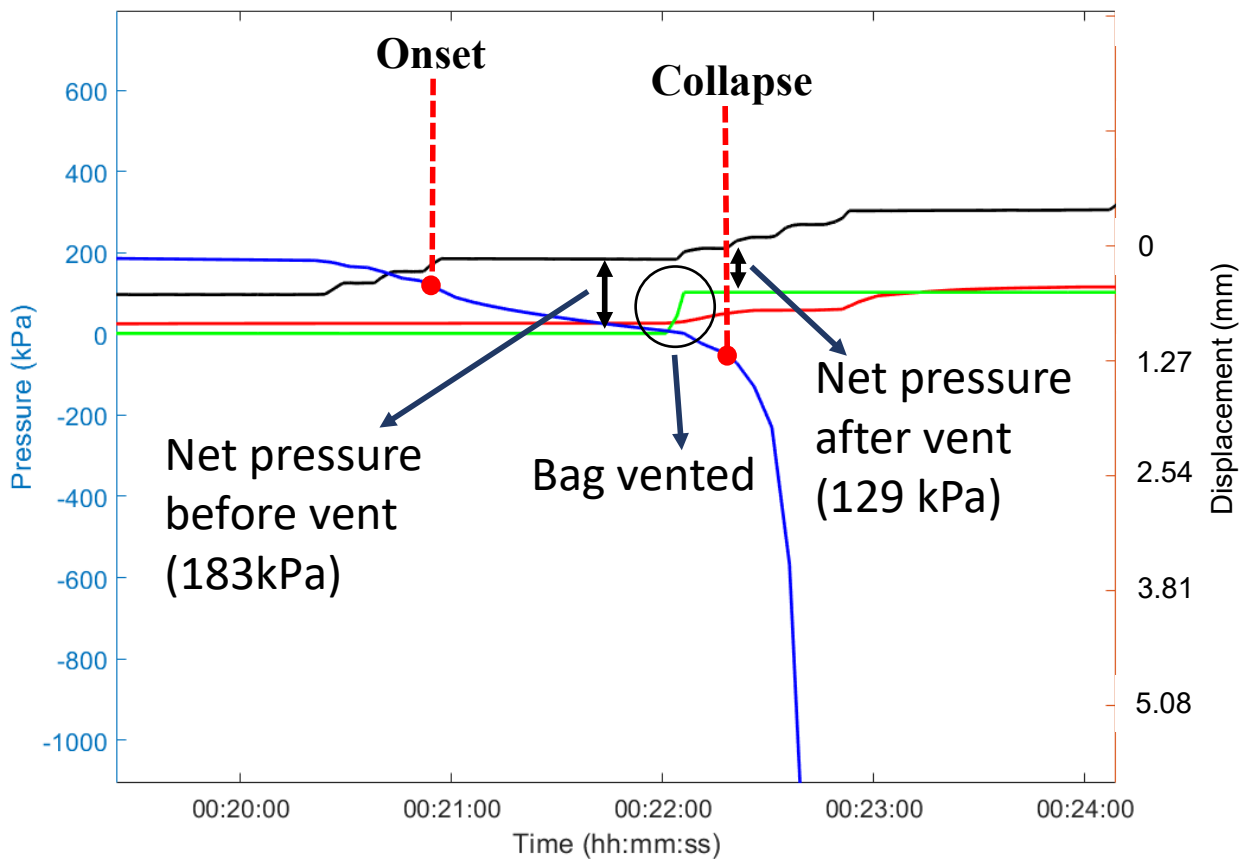

Figure 5.15: In-situ data from $45^{\circ}$ core test. Collapse occurs immediately after venting of vacuum, following a one minute hold at higher pressure.

the vacuum bag. In this instant, the net pressure is actually reduced to $129 \mathrm{kPa}$. The pressure prior to venting $(183 \mathrm{kPa})$ had been held for one minute. It is likely that the higher pressure state prior to venting was responsible for core movement and is therefore the value used in Figure 5.14. This suggests a time dependent response as the core was likely on the verge of collapse and reducing the net pressure did not delay failure. This is seen in Figure 5.15. 


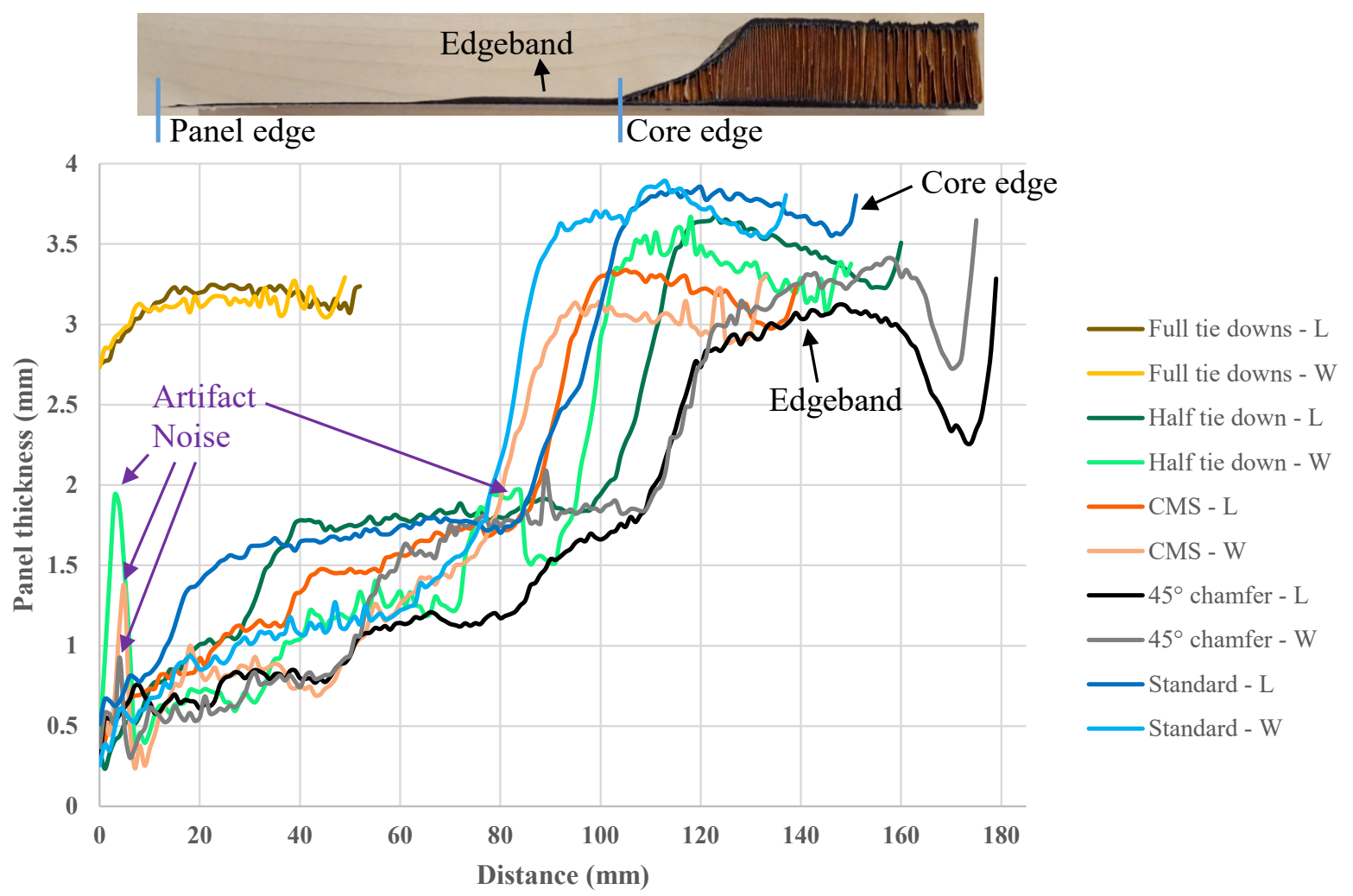

Figure 5.16: Change in thickness across laminate edge of processed panels in the $\mathrm{L}$ and $\mathrm{W}$ directions for varying layups. The full tie down cases show no externally visible core movement.

\subsubsection{Ply movement}

\section{Gross ply movement}

As in the two previous sections, the panel thickness across the laminate edge for each of the samples is displayed in Figure 5.16. Recall Figure 5.3 when interpreting the figure. The full tie down case provides a reference for a sample that does not exhibit core movement. Some artifact noise is seen which can be ignored. Again, measurements of thickness include that of the paint layer, which is not necessarily even among samples, resulting in variability between tests.

Thickness changes are similar amongst samples, indicating a similar pattern of core movement. The $45^{\circ}$ chamfer test is an exception, however. Several step-like changes in thickness are observed along the laminate edge, indicating a cascading effect has occurred. This is associated with considerable relative ply movement due to slippage across multiple prepreg-prepreg interfaces.

In each case a depression in thickness is seen just in front of the core edge. A similar pattern was observed in the previous tests, further suggesting that ply compaction is greatest at the edgeband transition. The depression observed in the $45^{\circ}$ chamfer test is signifantly larger than those of the other samples,. Microscope images revealed that this difference is the result of the core moving relative to the plies to the extent that filler plies are no longer in contact with the core. This creates a ply drop off in 
front of the core edge.

\section{Specific ply movement}

Individual ply movement maps are presented in Figure 5.17. In all cases, ply 1 remains stationary while all other plies slide to varying degrees. This matches that seen in Section 5.1 for samples processed at $120^{\circ} \mathrm{C}$.

Half tie downs show similar results to the standard layup despite a greater resistance to crush as previously shown. For the CMS sample, the bagside plies move more than the equivalent toolside plies. This is particularly true in the ribbon direction. This may be due to a weaker bond between plies and core along the toolside due to stabilization. Typically as the panel is heated, the film adhesive will form fillets with the core cells. This acts to provide a good bond between core and ply. Similarly pillowing of plies into the core may occur. While not ideal for mechanical properties [10], it likely increases the friction between core and ply. Therefore, if the core moves, the plies will likely move with it. However, if the toolside is stabilized, fillets cannot form and pillowing will not occur. Therefore, less friction acts on the toolside plies as the core moves inward. In short, CMS may result in a decrease in frictional resistance between ply and core along the toolside. In fact, in the non-ribbon direction, the core actually displaces $7 \mathrm{~mm}$ relative to the underlying plies. This is evidenced by the fact that filler plies 5-6 no longer butt up against the core. Interestingly, filler plies 7-8, which extend slightly up the core, don't seem to move much, if at all, in the microscope images (Figure 5.18). This confirms that the toolside frictional bond between core and prepreg has been weakened through stabilization. Therefore, for unstabilized layups, the frictional bond between core and prepreg can be assumed to be considerably higher than other frictional interfaces. That is, friction between prepreg layers, friction between the prepreg and bag (release film), and friction between the prepreg and tool (surfacing film). This raises the question that if tie downs were used in combination with CMS, would resistance to core movement increase or decrease as compared with only using tie downs? On one hand, the core chamfer is stiffer. However, on the other hand, the plies are now restrained yet the frictional resistance between ply and core on the toolside is less. In this scenario the core may simply deform relative to the plies.

The $45^{\circ}$ core sample displays considerable relative movement between plies. The plies immediately surrounding the core (plies 4-9) experience the largest displacement. It is possible that plies 4-9 initially slipped across the ply $9 / 10$ and 3/4 interfaces. As these plies slid, the hydrodynamic friction acting on the neighbouring plies would have increased; thus dragging further plies inward, resulting in a cascading effect. Just as in the CMS-W sample, a gap is seen between the core and filler plies 5-6, indicating deformation of the core relative to the plies. In this sample however, the core moved relative to both toolside and bagside plies. This is observed in the microscope images (Figure 5.19), as plies 7-8 are no longer at their original position along the core (6.35 and $12.7 \mathrm{~mm}$ up the chamfer edge respectively). In fact, in the L direction, the relative displacement of the core with ply 7 is such that the ply no longer contacts the core. Ply 8 only just maintains contact with the edge of the core (Figure 5.19-L). Similarly, in the $\mathrm{W}$ direction ply 7 only just maintains contact with the core. The displacement of the core edge relative to the plies was approximately $15 \mathrm{~mm}$ in the $\mathrm{L}$ direction and $8 \mathrm{~mm}$ in the $\mathrm{W}$ direction. Such 
| Core edge before movement

I Core edge after movement

- Ply movement

W

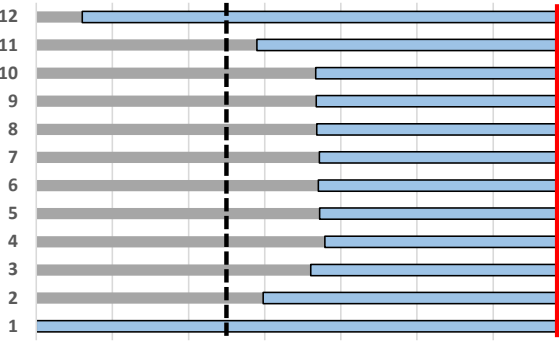

$\square$ Final ply position

$\mathbf{L}$

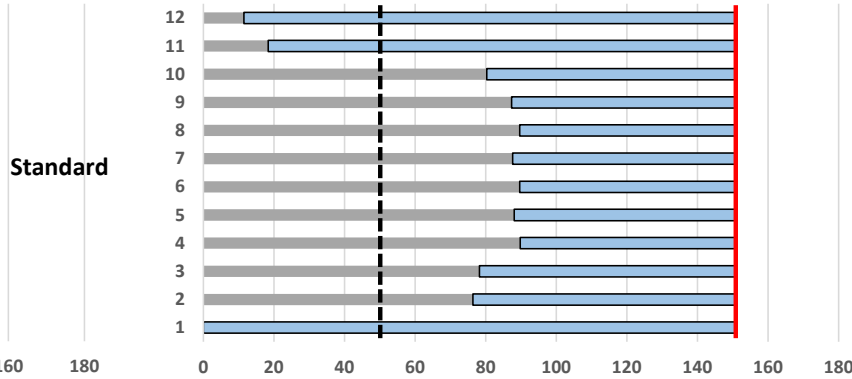

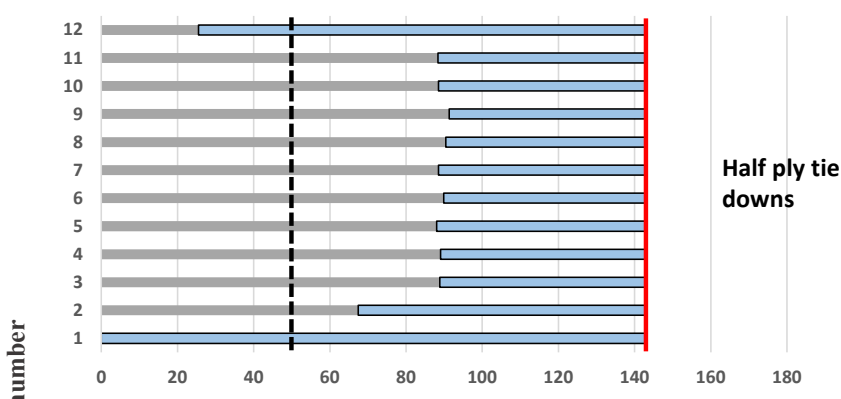

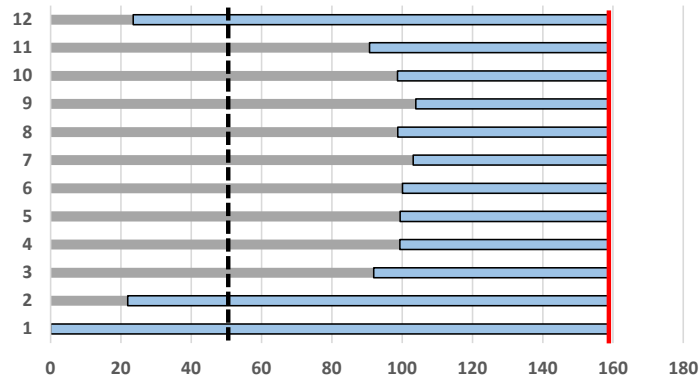

$\frac{2}{2}$
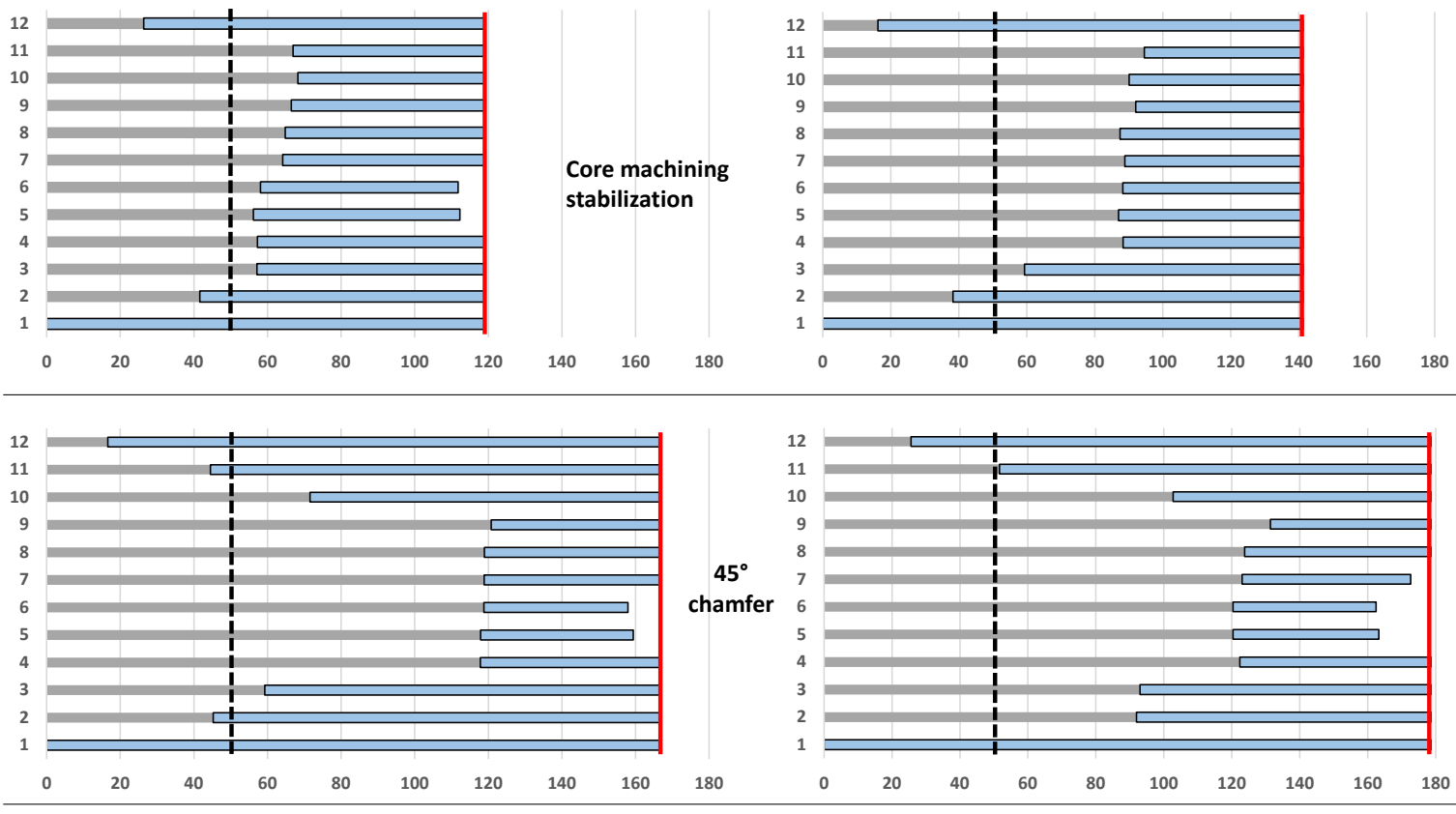

Distance from panel edge ( $\mathrm{mm})$

Figure 5.17: Specific ply movement profile of processed panels in $\mathrm{L}$ and $\mathrm{W}$ for varying layups. Initially, the core edge is $50.8 \mathrm{~mm}$ from the panel edge. 


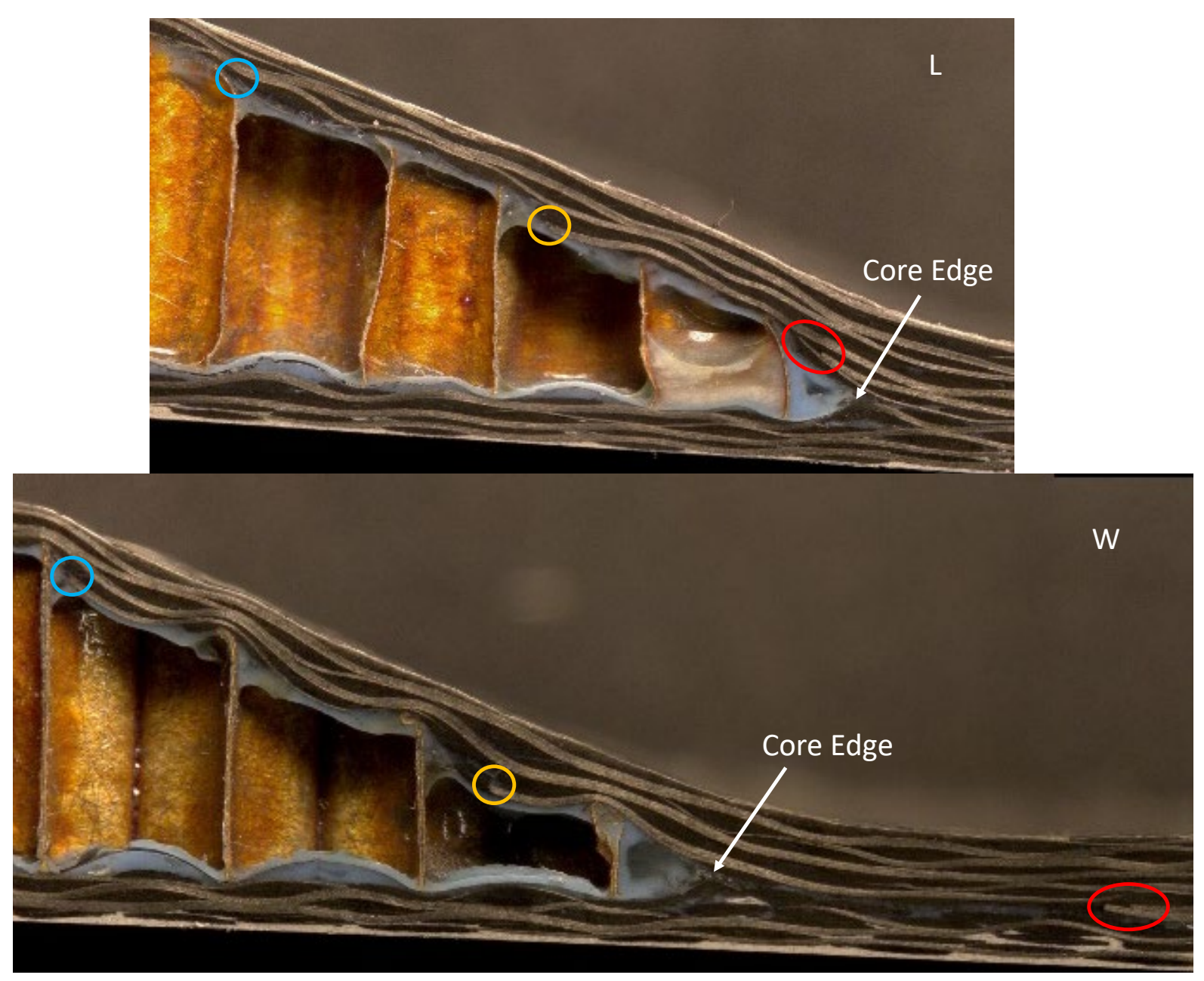

Figure 5.18: Microscope images showing termination of plies 5-6 (red circle), ply 7 (yellow circle), and ply 8 (blue circle) in the $\mathrm{W}$ and $\mathrm{L}$ directions for the CMS sample.

relative deformation was not observed in any of the other samples except in the case of CMS Hence it can be said that while the frictional interface between core and prepreg can be ignored for chamfer angles of $20^{\circ}$ and less, the interface must be considered for steeper chamfer angles.

\subsubsection{Extent of core movement}

Images of each sample post processing are shown in Figure 5.20. The extent of core movement for each sample is outlined in Table 5.4. This represents the distance the core moves in from a single side. This is determined by subtracting the original position of the core from its final, post-cure, position.

Generally speaking, samples that exhibited a higher onset pressure as compared with the standard layup also exhibited a lower degree of core movement and vice versa. The half tie down test is an exception, where onset occurs at a higher pressure than the standard test, yet the extent of core movement is also greater. Unlike in the previous section, DOC does not play a role in dictating the extent of crush. Therefore, propagation of the crush front is allowed to continue until a high enough level of densification 


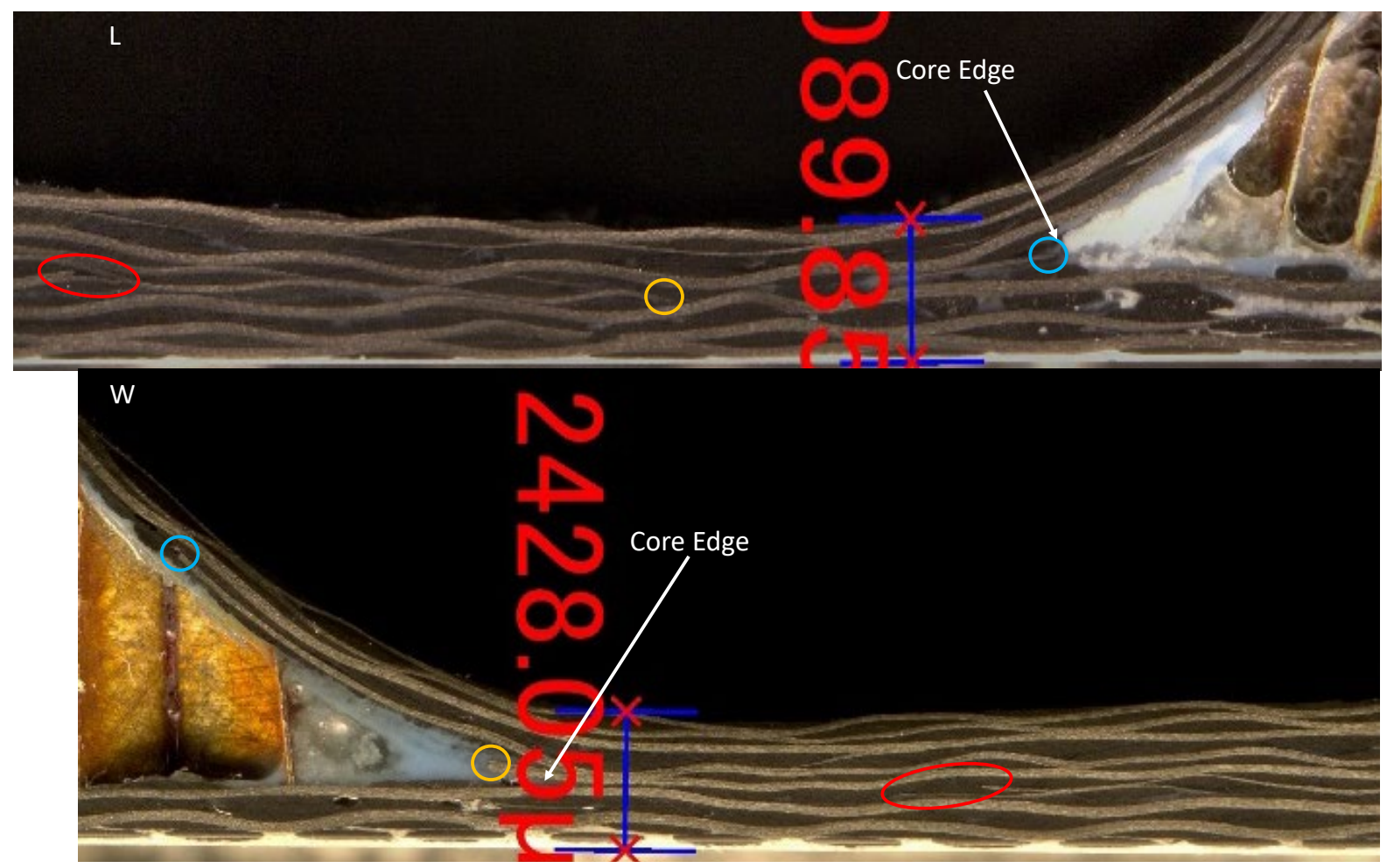

Figure 5.19: Microscope images showing termination of plies 5-6 (red circle), ply 7 (yellow circle), and ply 8 (blue circle) in the $\mathrm{W}$ and $\mathrm{L}$ directions for the $45^{\circ}$ chamfer sample.

is achieved that the resistive forces outweigh the applied force. In the case of the half tie down test, crush is only occurring along adjacent edges. Hence, the crush front can continue further in a single direction since the cells far away are not simultaneously being crushed from the opposite direction, as would be the case for the other tests. In other words, the edges experiencing core movement must compensate by crushing further to achieve a similar level of densification that would be present if opposing edges were crushing together.

In the CMS sample, it was observed that the stabilized region experienced little to no core movement. CMS effectively limited the area that is able to crush to that of the unstabilized region. This resulted in a smaller area to densify, thus reducing the extent of movement.

With a steeper chamfer angle, the horizontal component of pressure is much greater. Therefore a higher level of densification is required to arrest the crush front, resulting in a higher degree of core movement. Hence, why the $45^{\circ}$ sample displayed the highest level of crush.

Recall that the section cuts used to obtain the individual ply movement profiles were not always the exact center of the panel. Therefore, the extent of core movement displayed in Figure 5.17 may not align exactly with that provided in Table 5.4.

Once again, the ribbon direction exhibits greater crush than the non-ribbon direction. This further supports the notion that homogeneous, buckling of cells in the L direction is preferential to plastic collapse in the $\mathrm{W}$ direction. As shown previously, a homogeneous collapse pattern as seen in the $\mathrm{L}$ direction, seems to progress through the panel at a faster rate; thus dominating the response. Unfortu- 


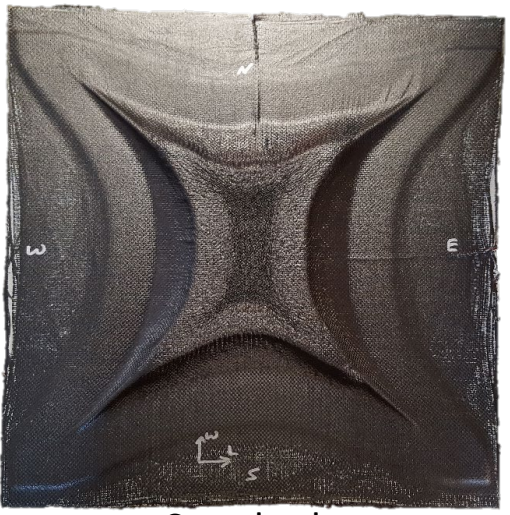

Standard

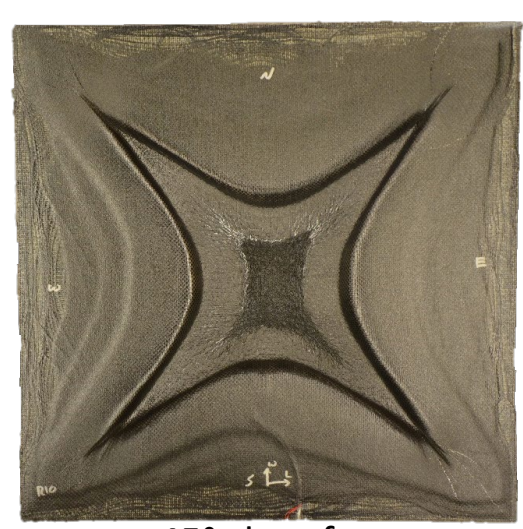

$45^{\circ}$ chamfer

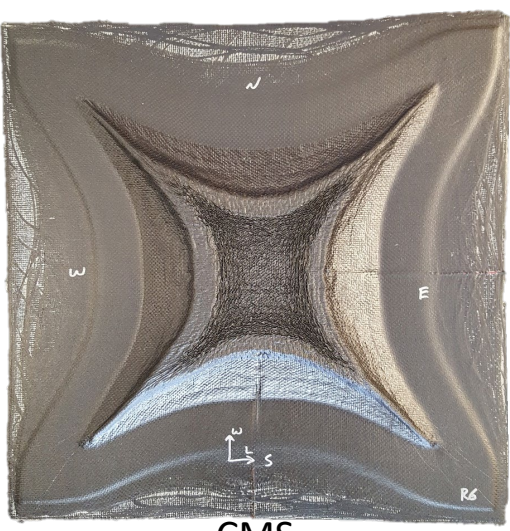

CMS

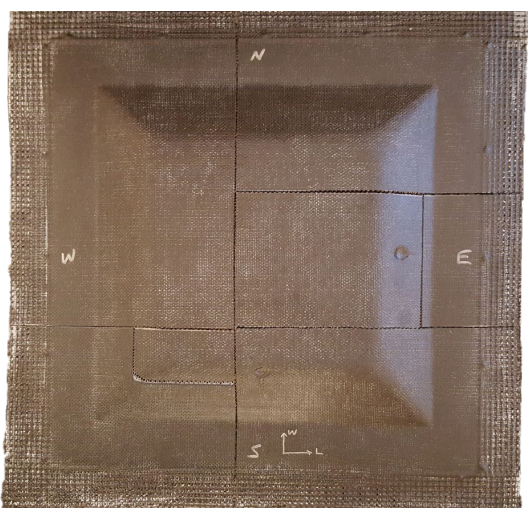

Full tie downs

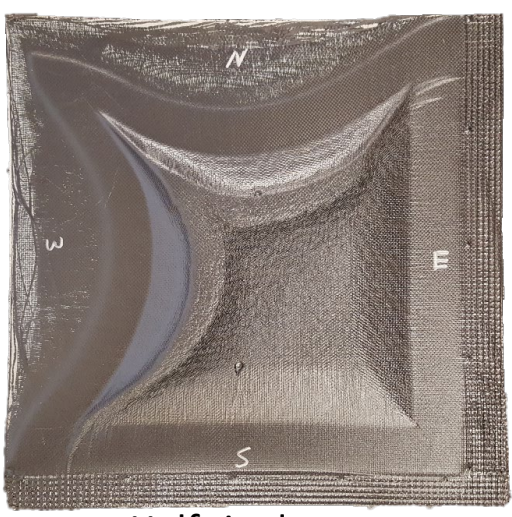

Half tie downs

Figure 5.20: Standard, full tie down, half tie down, CMS, and $45^{\circ}$ chamfer tests post-processing, showing extent of core movement.

Table 5.4: Extent of deformation in the ribbon and non-ribbon direction associated with various layup features.

\begin{tabular}{|c|c|c|}
\hline Test & Displacement in L $(\mathbf{m m})$ & Displacement in W $(\mathbf{m m})$ \\
\hline Standard & 100 & 86 \\
\hline Half tie downs & 109 & 99 \\
\hline CMS & 88 & 82 \\
\hline $45^{\circ}$ chamfer & 128 & 124 \\
\hline
\end{tabular}

nately due to failure of one or more of the LVDT during processing, the rate of progression of core movement in the $\mathrm{L}$ and $\mathrm{W}$ directions could not be compared. 


\subsection{Dry core test}

The purpose of this section is to gain a basic understanding of the behaviour of honeycomb core movement on its own, in the absence of plies (dry honeycomb). The test results come from the "Dry Core Test" outlined in Section 4.8 .

\subsubsection{Failure pressure}

Movement of the core was noticeable almost immediately after external pressure application, at a net pressure of $111 \mathrm{kPa}$. This is labeled as the onset point. Collapse occurs at $147 \mathrm{kPa}$, after which the rate of deformation increases further. This is shown in Figure 5.21. While the numbers are indicative of the resistance to core movement offered by the core, the experiment does not mimic a compression test. Since the core is sliding across the tool under external pressure, there is a friction force that resists movement. That said, the PTFE release film under the core reduces the magnitude of friction with the tool. However, the maximum deformation observed was less than the typical deformation in the sandwich panels tests, suggesting that the core-tool friction is still considerable. The core and release film slide together over the tool (as observed from the video footage). The core was not see to crush solely under vacuum pressure.

Surprisingly, the core recovers almost entirely upon depressurization (Figure 5.22). In fact, the $\mathrm{L}$ direction displayed perfect elasticity with only minor plastic deformation observed in $\mathrm{W}$ along the chamfer radius (Figure 5.23). This indicates that the core cells can experience large strains (up to densification) while remaining elastic in nature. Therefore, the plateau stress (stress at cell collapse) is not synonymous with an ultimate compressive strength. It follows then that honeycomb stiffness rather than strength is of importance in regards to core movement. It should be noted that, in sandwich panels, core movement is non-recoverable due to curing of the plies. This raises the notion that perhaps core movement initiation should not be thought of in terms of a critical force, but rather in terms of a failure strain.

Unfortunately, in-plane properties of the honeycomb are not provided on data sheets by Hexcel [24, 25]. However, studies have been performed [21] which determined the Young's Modulus of NOMEX ${ }^{\circledR}$ paper to be on the order of $3.1 \mathrm{GPa}$. In the experiments presented here, the honeycombs had an approximate cell size and thickness of 3.175 and $0.08 \mathrm{~mm}$ respectively. Therefore, using Equation 2.4, the in-plane Young's Modulus of the honeycomb is approximately 0.6 MPa. Similarly, the plateau stress can be calculated using Equation 2.8. This gives a plateau stress of $57 \mathrm{kPa}$. Thus, it does not require much stress to significantly deform the honeycomb. This is corroborated by the experimental results above. Although the experimental values are double the analytical results (111 kPa vs 57 $\mathrm{kPa}$ ), there is likely a significant friction component that affects the experimental results. Moreover, the analytical results assume uniaxial compression, whereas in reality, biaxial affects may influence deformation. 


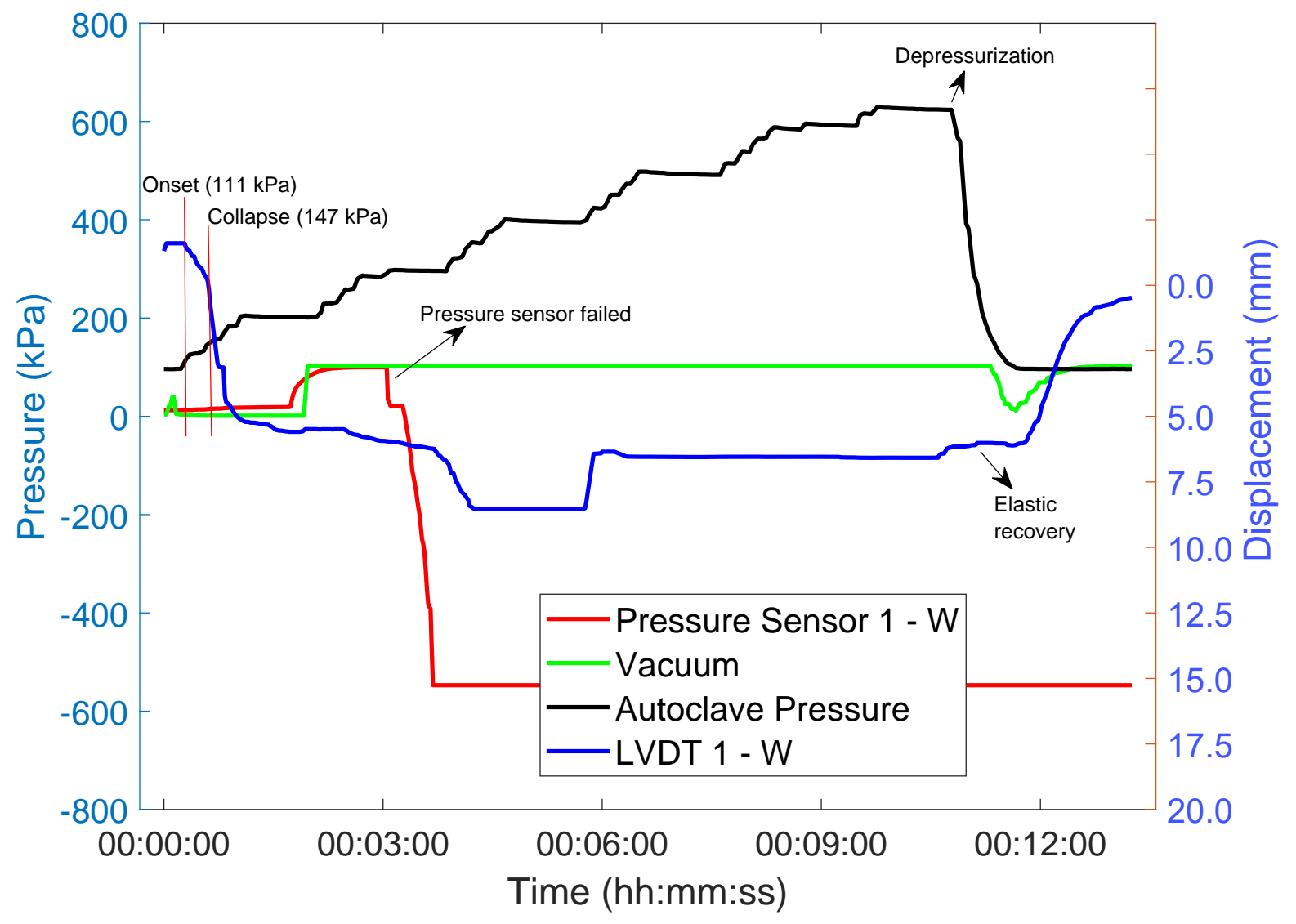

Figure 5.21: In-situ results from dry core test. Cells deform upon loading and recover almost entirely upon unloading. 


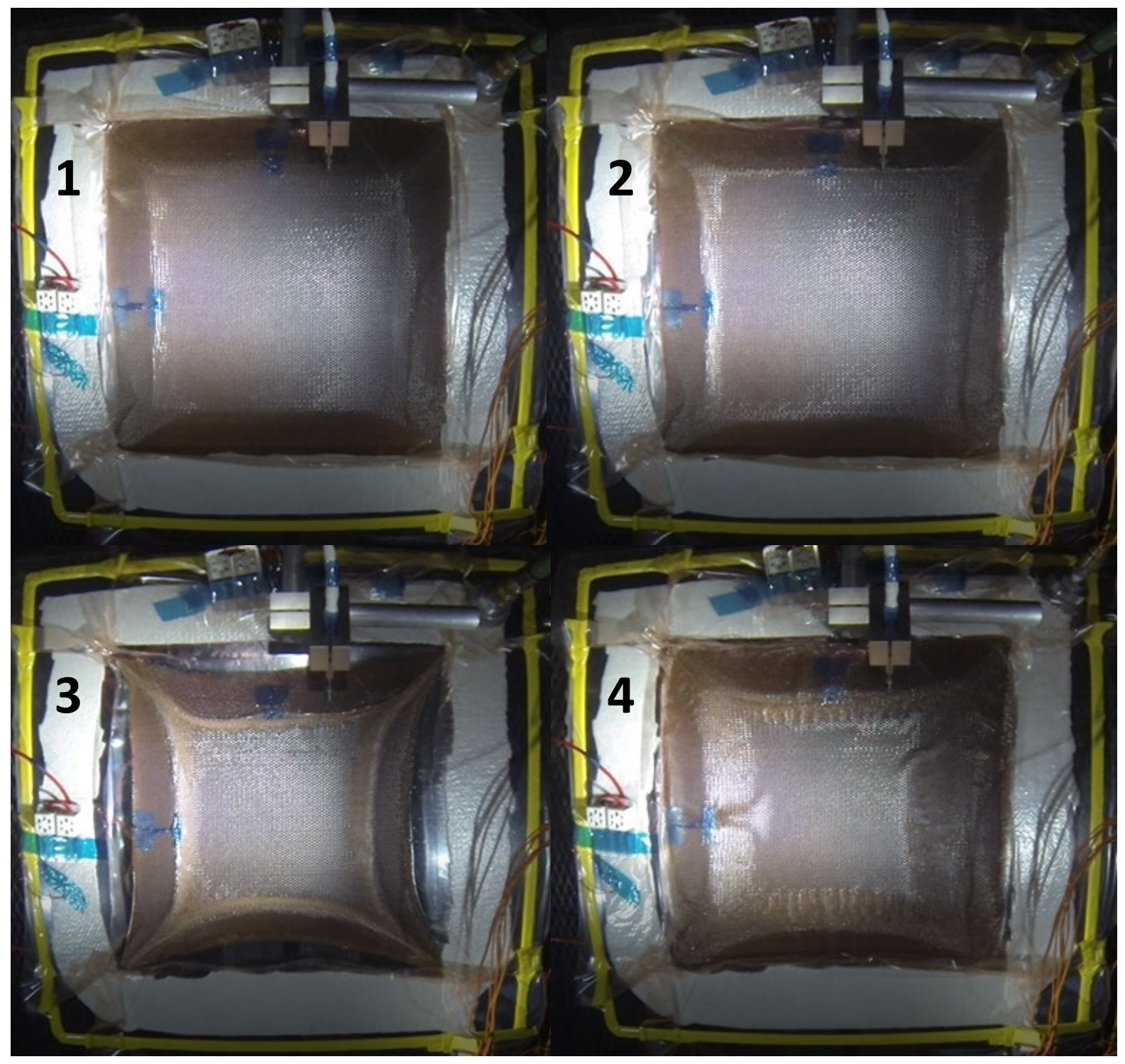

Figure 5.22: Snapshots taken during autoclave test for dry honeycomb. 1: Prior to pressure application. 2: Moment of collapse. 3: Maximum deformation. 4: Fully unloaded honeycomb core following depressurization. 

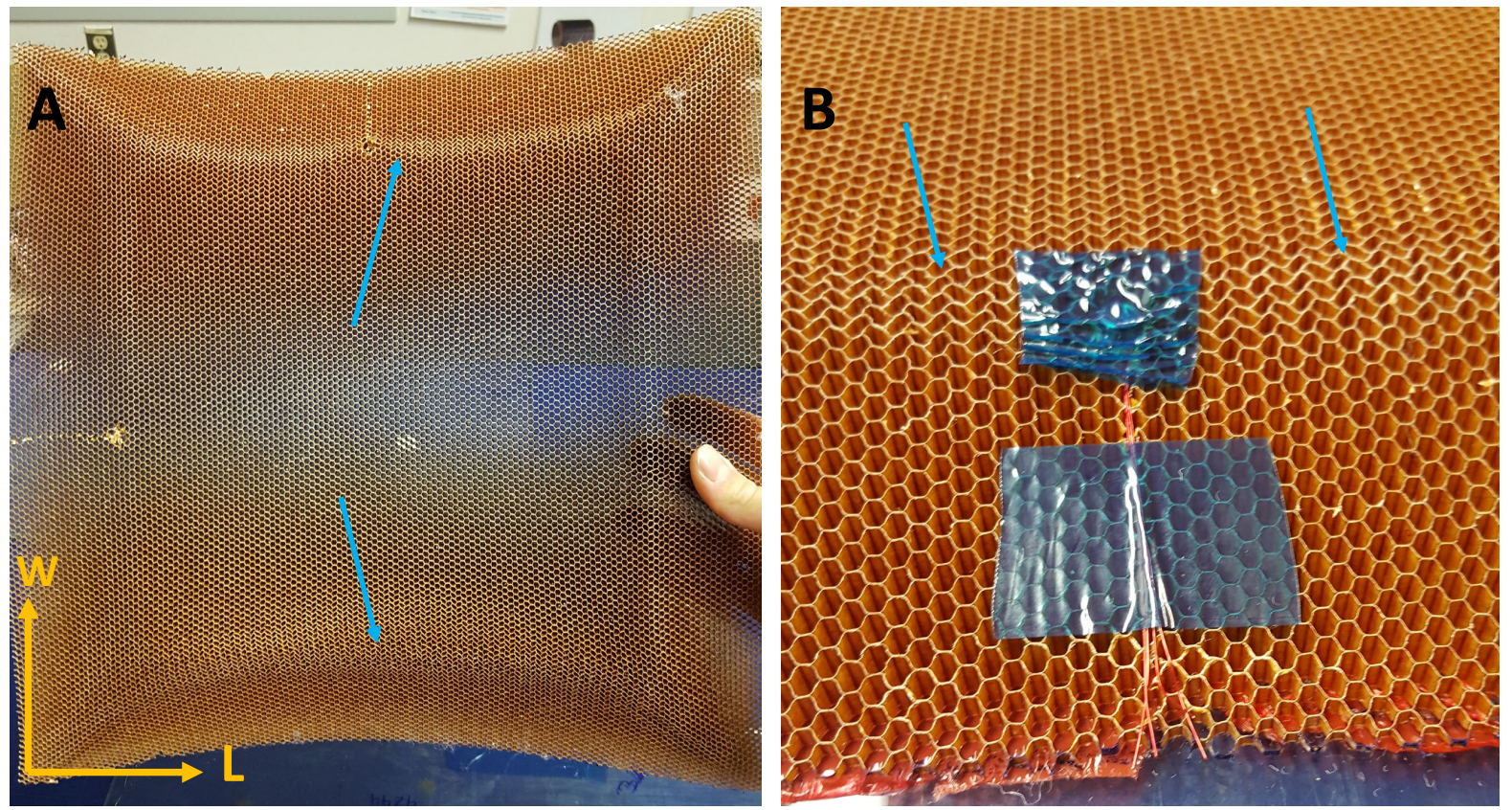

Figure 5.23: Plastic deformation in $\mathrm{W}$ following autoclave pressurization. (A) Image of entire honeycomb. (B) Magnified view of deformation in $\mathrm{W}$. 


\subsection{Core movement mechanics}

The following section details observations regarding the initiation and progression of core movement. These observations along with results from the previous sections provide the basis for an updated mechanical model.

\subsubsection{Initiation of core movement}

During the bending phase of honeycomb compression, the entire structure exhibits this response. The same pattern presents itself during the initiation of core movement. As the cellular structure bends, the prepreg similarly deforms until eventually, plies are dragged inward with the core and wrinkles form. The initial deformation of the prepreg creates a rougher surface which is picked up by the video camera and LVDT during onset. Figure 5.24 provides an example of the change in surface roughness of the panel, seen through the breather cloth, prior to collapse. The images in the figure come from the in-situ video footage.

The change in surface roughness associated with bending of the honeycomb structure is sensitive to minute deformations. In the case of the full tie down test, core movement was not detected, however small distortions in the cellular pattern were observed from in-plane section cuts as shown in Figure 5.13. The surface roughness of the tie down test is notably different to the touch from that of the room temperature test where no distortion in the cellular pattern was seen. This infers that slight alterations in the cellular pattern similarly present themselves in the prepreg surface. However, minor bending is not easily detectable in-situ. Haptic feedback may prove a possible means of detection, but is limited to post-processing assessment. It is also possible that rearrangement of the LVDTs sensors would allow for such small deformations to be picked up. That said, aside from very minute distortions, the experimental setup described here provides a reliable means to detect core movement during the early stages of the process.

Figure 5.25 provides a comparison of in-plane section cuts showing different levels of core movement during initiation. The first two images are of the room temperature sample displaying no core movement, and the full tie down test showing minor bending. The latter two images are of the half tie down test displaying considerable bending and of the $180^{\circ} \mathrm{C}$ test showing early stages of collapse. Recall that the $180^{\circ} \mathrm{C}$ test exhibited relatively little core movement in comparison with the other samples due to a rapid advancement of DOC. The level of densification is significantly more advanced in the $\mathrm{L}$ direction than in the $\mathrm{W}$ direction as mentioned in Section 5.1.3. The image provided for the half tie down test is between the two restrained sides. Note that the scale of bending in this sample is greater than in the full tie down test, showing that movement along the unrestrained edges influences deformation along the restrained edges. Ideally, images would come from the same sample at different points along the processing window. However, due to the nature of the experiments this was not possible. As a result, varying extents of deformation are observed through different experiments. 

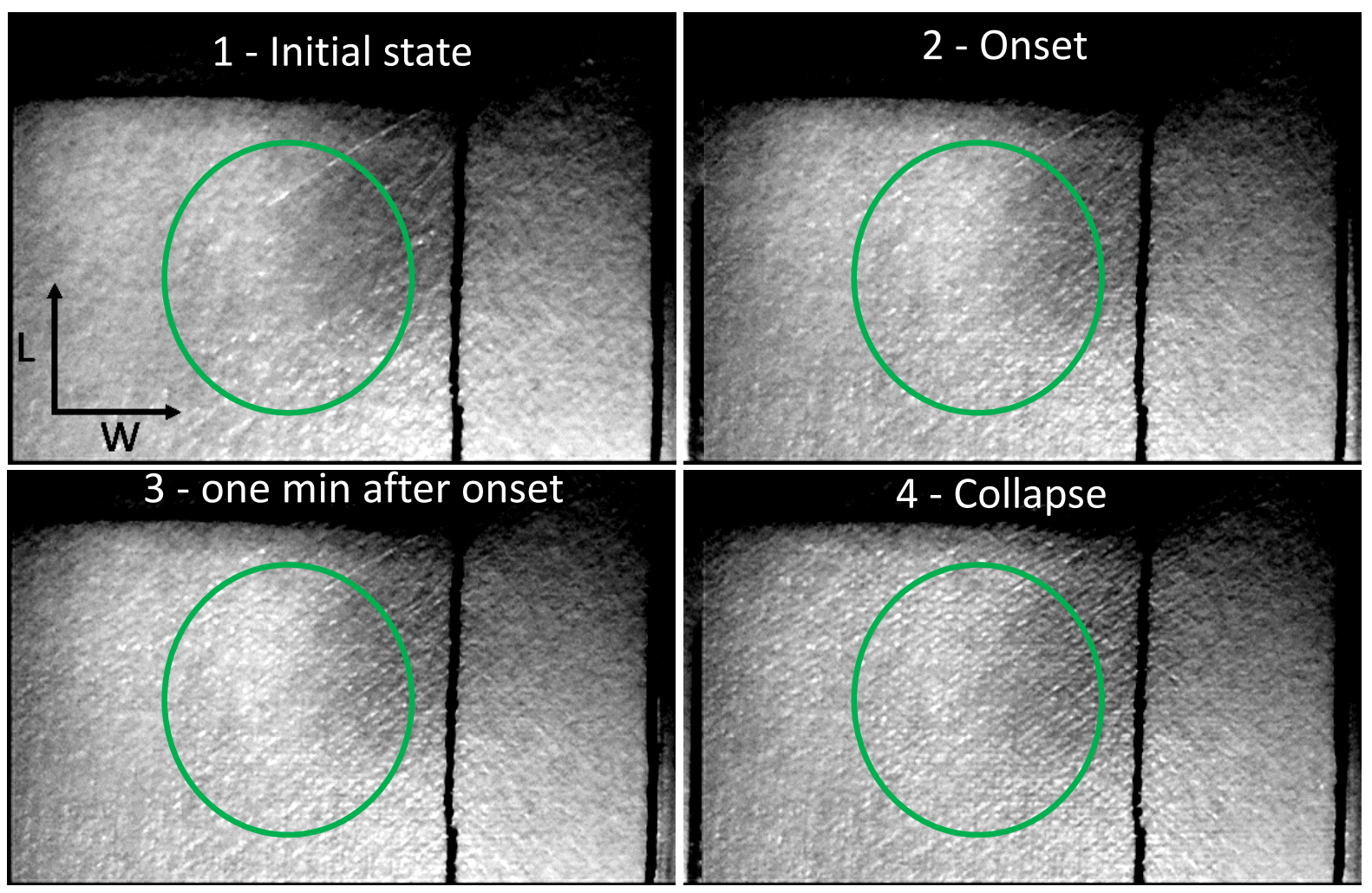

Figure 5.24: Change in surface roughness from onset of core movement through to collapse. Transitioning from 1-4, a notable change in surface roughness is seen within the green circle. The bagside plies conform to the honeycomb structure as it deforms, observable through the breather cloth.

\subsubsection{Progression of core movement}

\section{Collapse pattern}

Data from CMM scans allowed for a gradient map displaying slope angle to be created for each sample. This data is presented in Figure 5.26. The maps gives an indication of where core movement occurs. Artifact noise, presenting itself as a sudden line across the sample, is seen in a few of the images and should be disregarded. The images in the figure are representative of the experiments performed in Section 5.3, as the variation in layup results in an altered mechanical response. The experiments performed in Section 5.1 and Section 5.2 show similar results to the standard layup experiment, with the primary difference being the magnitude of core movement. The slope-gradient maps for each sample are provided in Appendix B

Near the chamfer radius, the angle of the chamfer drastically increases. Further along the chamfer edge, however, the slope angle is largely unchanged, indicating crush does not occur along the lower portion of the chamfer edge. This was also observed in experiments by Alteneder et al [6] and mentioned as being a reoccurring theme by Renn et al [42]. This effect is particularly true for the CMS sample, where the angle of the chamfer remains at $20^{\circ}$ except for right at the radius, where there is a steep 


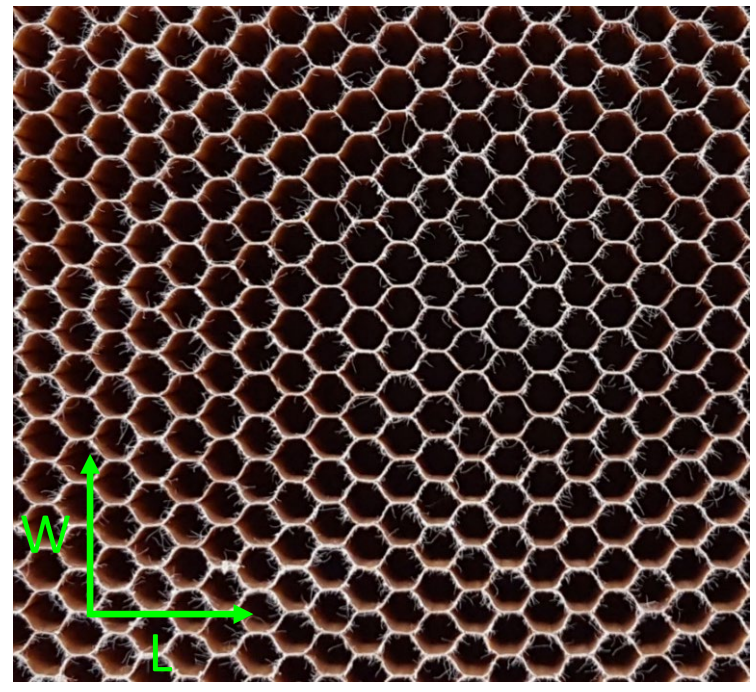

(a) No core movement - room temp.

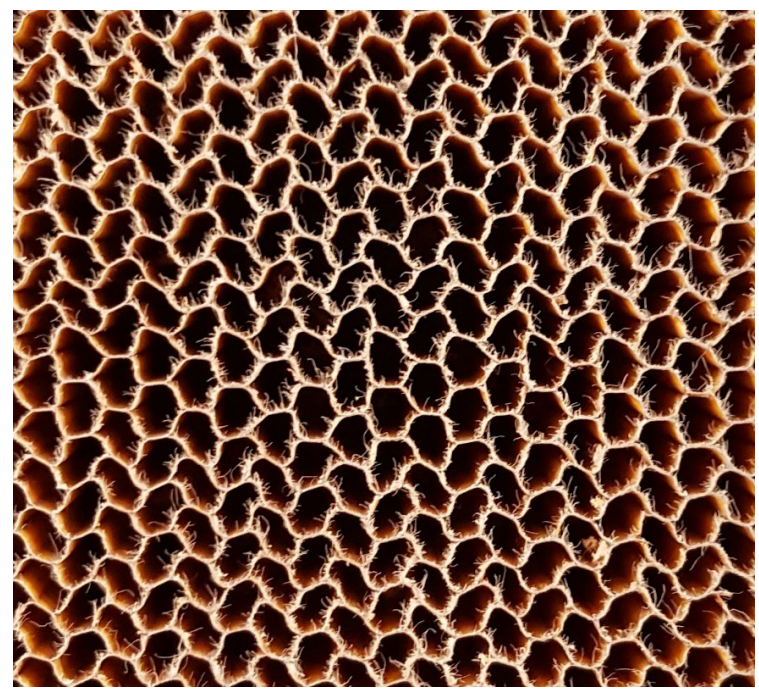

(c) Bending - half tie downs.

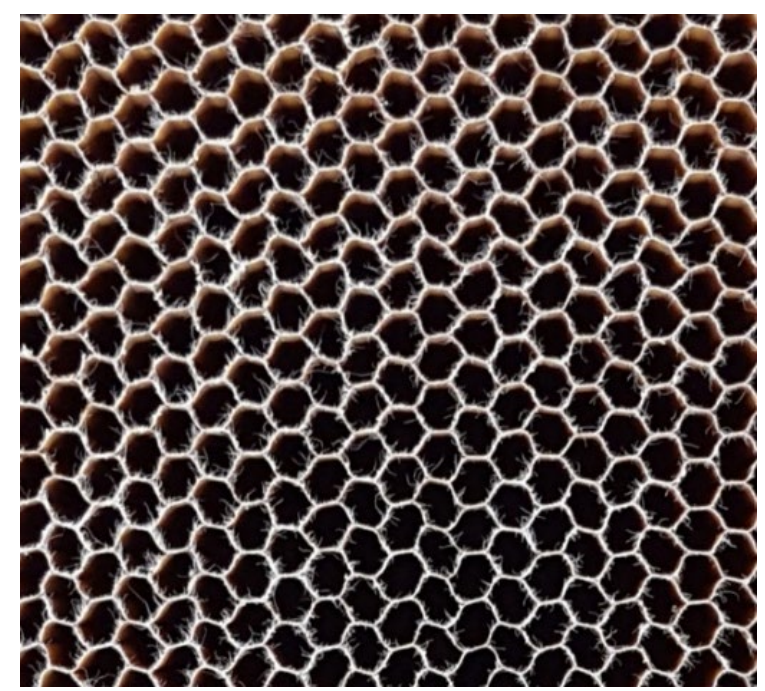

(b) Minor bending - full tie downs.

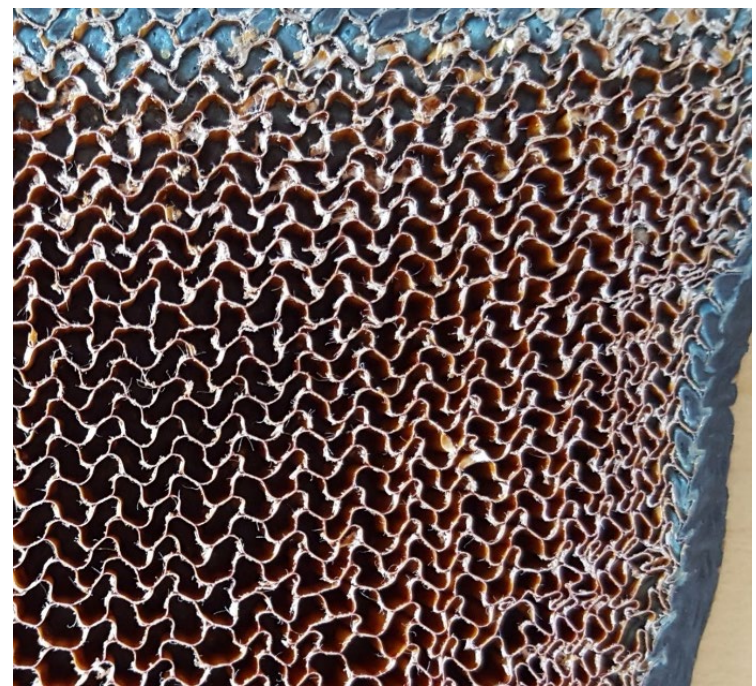

(d) Collapse $-180^{\circ} \mathrm{C}$.

Figure 5.25: In-plane initiation of core movement showing the progression of cell bending throughout the honeycomb. Images are from different tests showing (a) no core movement, (b) minor bending, (c) widespread bending, and (d) early stages of collapse. 


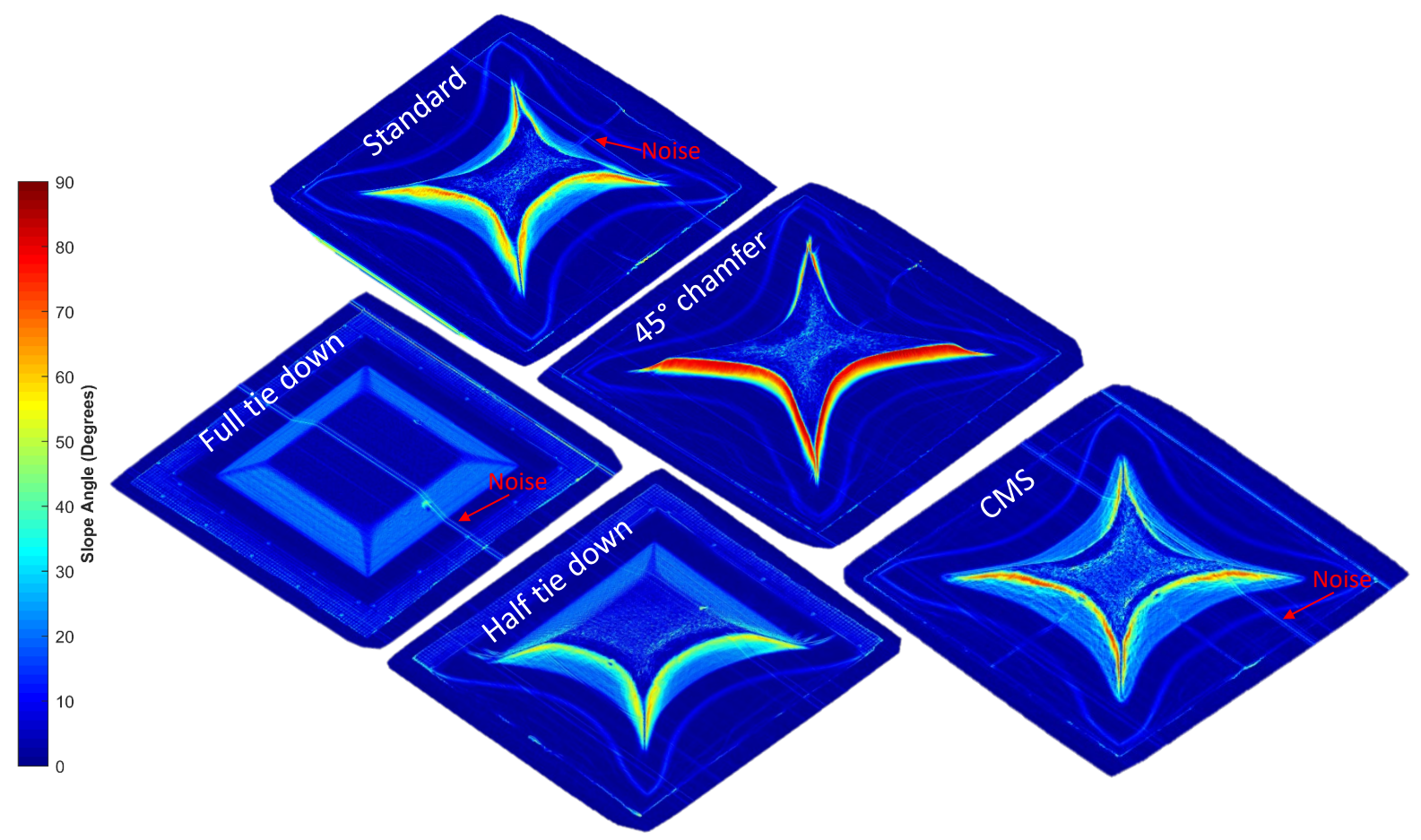

Figure 5.26: Slope angle of processed panels. Dark red indicates a vertical slope with an angle of $90^{\circ}$. Dark blue indicates a flat plane with an angle of $0^{\circ}$. For most samples, a slope angle greater than $20^{\circ}$ indicates crush has occurred along the chamfer edge. For the $45^{\circ}$ chamfer, a slope angle larger than $45^{\circ}$ indicates crush.

increase in slope. This shows that CMS restricts core movement from occurring within the stabilized zone. The unstabilized zone remains susceptible to crush, however. The sample with a $45^{\circ}$ chamfer displays a different result. In this test a drastic increase in slope is seen immediately, indicating that crush occurs along the majority of the chamfer edge if the chamfer is steep enough.

In most samples, notable movement initiates as the panel begins to crush at the chamfer radius. The crush front (i.e. the edge of the crush zone) propagates through the flat section of the panel and the core moves inward like an accordion. As the chamfer edge pulls in, it too crushes near the radius and the crush zone extends further down the chamfer edge. This creates a change in slope along the chamfer edge. With CMS however, crush does not originate at the chamfer radius. Rather, it originates beyond the stabilized zone. As the crush front progresses, the chamfer edge pulls inward resulting in crush at the radius. However, the crush zone does not extend down the stabilized chamfer edge. Therefore, the chamfer angle remains relatively constant. The difference in movement pattern during core movement for the standard layup versus CMS are displayed in Figure 5.27a and Figure 5.27b respectively. The images in the figures come from the in-situ video footage of the $95^{\circ} \mathrm{C}$ and $\mathrm{CMS}$ samples.

Figure 5.27a shows a gradual increase in the size of the crush zone as the crush front progresses inward from the chamfer radius toward the center of the panel. In Figure $5.27 \mathrm{~b}$ the crush front originates $16.5 \mathrm{~mm}$ from the chamfer radius. Stabilization extends $6.41 \mathrm{~mm}$ past the chamfer radius meaning that the crush front originates $10.1 \mathrm{~mm}$ past the stabilized area. The crush front is observed through the 


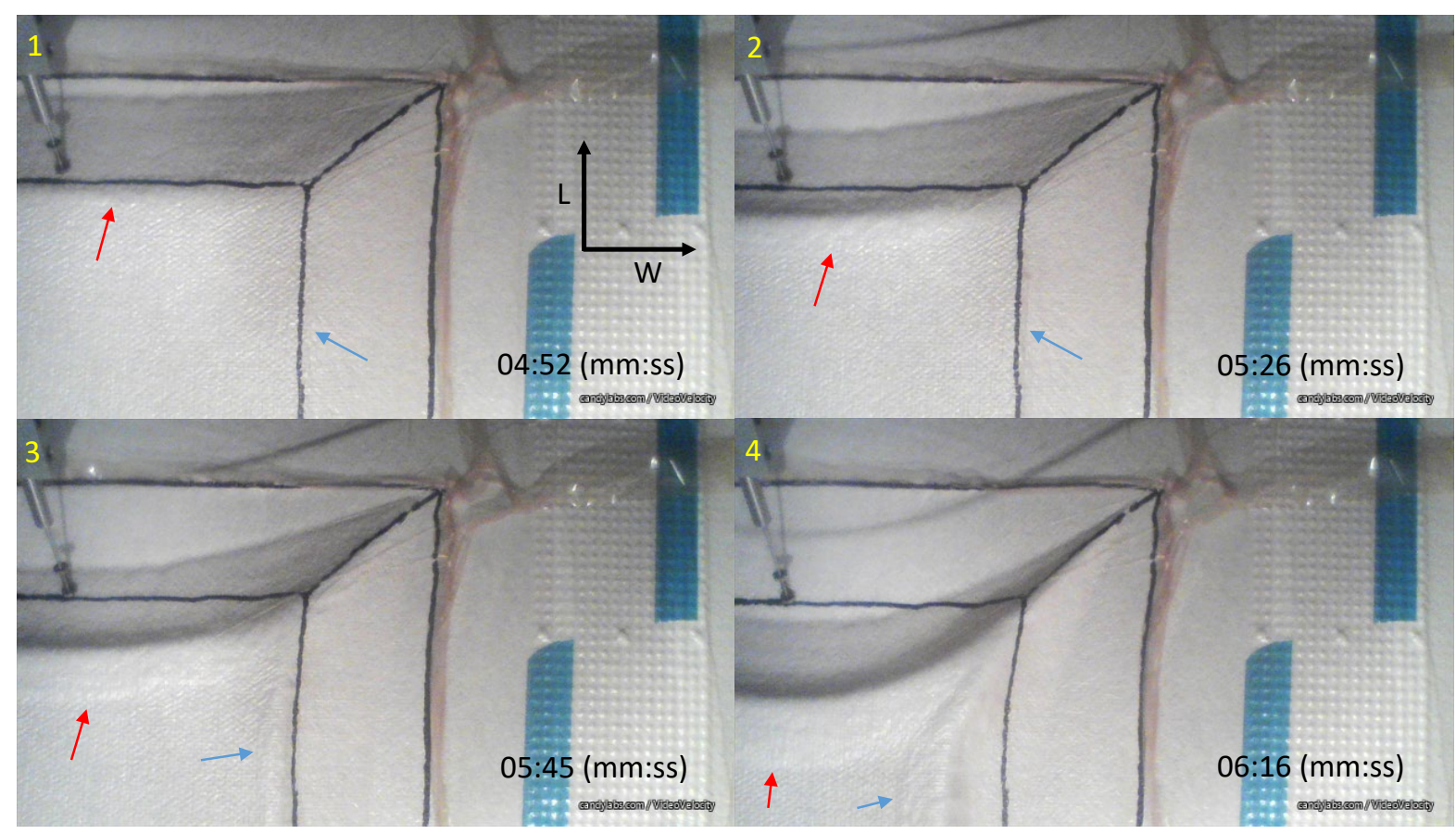

(a) Standard crush pattern $-95^{\circ} \mathrm{C}$ sample

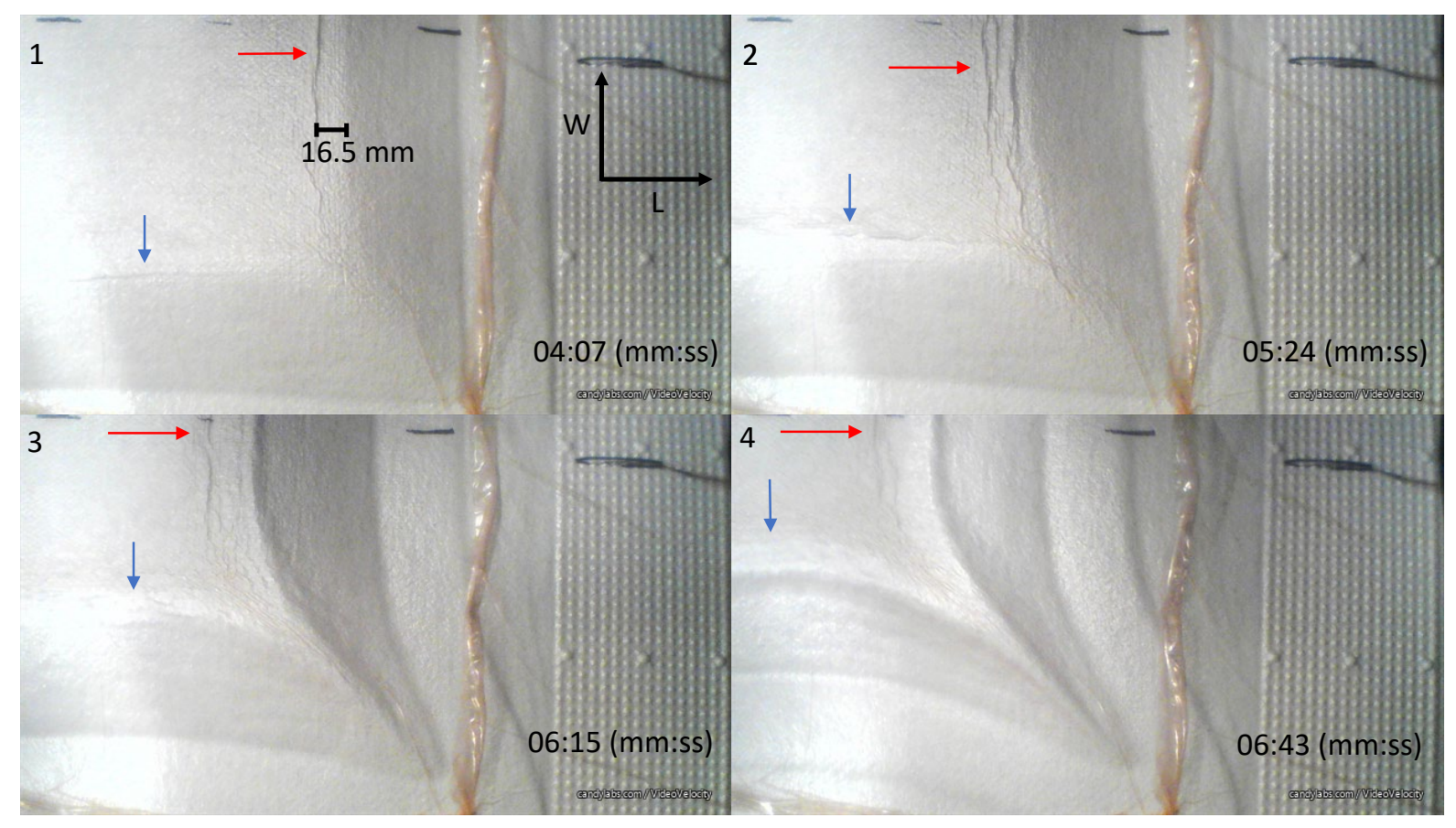

(b) CMS crush pattern.

Figure 5.27: (a) Standard and (b) CMS crush patterns, displaying growth of the crush zone from image 1-4. Red arrows show the crush front in the L direction while blue arrows show the crush front in the $\mathrm{W}$ direction. Elapsed time from the application of pressure is displayed. Note the change in orientation between samples. 
formation of a large bagside wrinkle (image 1). Initially, as the crush front progresses, many wrinkles form within the crush zone (image 2). However, eventually as the chamfer edge moves inward, the radius of the core crushes and the crush zone begins to smooth out as the wrinkles congregate past the crush front (image 3-4).

Contrast is poor in the $\mathrm{W}$ direction, however LVDT sensors indicate that, for all samples, onset and collapse occur simultaneously in the $\mathrm{L}$ and $\mathrm{W}$ directions. The video footage shows that the crush front progresses at a faster rate in the $\mathrm{L}$ direction as compared with the $\mathrm{W}$ direction, particularly in Figure 5.27a. That is not to say that the core necessarily moves inward quicker. Rather, in the L direction cells beyond the crush front seemingly undergo collapse before the current row fully densifies, thus extending the crush zone. However, in the $\mathrm{W}$ direction densification at the crush front seems to precede collapse in neighbouring rows. In both cases, however, the chamfer edge moves inward with cell displacement. In fact, for the $95^{\circ} \mathrm{C}$ sample, LVDT data indicates that movement in the $\mathrm{L}$ and $\mathrm{W}$ direction occur at a similar rate (Figure 5.7a). Unfortunately, this is difficult to distinguish in the video footage due to poor contrast in the $\mathrm{W}$ direction. This observation makes sense considering the manner of cell collapse in the two directions. The homogenous, buckling pattern in the L direction would allow for an extended projection of area susceptible to crush. On the other hand, the row-by-row collapse pattern in the $\mathrm{W}$ direction would only allow for the crush front to progress once the current row has densified. Both increase the magnitude of core movement, however the latter option results in a crush zone that grows at a slower rate. A similar result was presented by Heimbs et al [23]. It is reasonable to assume that this manner of crush progression would result in regions of higher densification in the $\mathrm{W}$ direction. Through-thickness and in-plane section cuts reveal this exact revelation and are presented below. Through-thickness sections of the $95^{\circ} \mathrm{C}$ sample are provided in Appendix B.

As observed in the $600 \mathrm{kPa}$ sample, it is possible for the $\mathrm{W}$ crush front to progress at a faster rate than the $\mathrm{L}$ crush front. This is shown in Figure 5.28. Again, the rate of core movement is similar among the samples, with the L direction actually showing a slightly quicker deformation response initially (recall Figure 5.12). This suggests that the crush front that progresses through the material quicker will result in a greater final magnitude of core movement in that direction. As shown, initially the rate of progression of the crush zone does not necessarily correspond to the rate of core movement due to the different collapse modes. However, it is likely that the crush front which progresses quicker will dominate the late stage response, allowing for greater core movement in that direction.

It should be noted that a faster crush front can also result in a faster rate of core movement initially. This was the case for the $120_{\text {orig }}^{\circ} \mathrm{C}$ and $180^{\circ}$ tests.

\section{Deformation of chamfer edge}

In the $120^{\circ} \mathrm{C}$, standard layup and $45^{\circ}$ chamfer experiments the LVDTs were placed along equivalent edges to track deformation of the chamfer edge itself. Moreover, a 12.7 x $12.7 \mathrm{~mm}$ grid pattern was implemented to measure the rate of displacement of the chamfer edge from the video footage. Only two tests of this caliber were conducted to see the difference in the rate of core movement for a chamfer angle of $20^{\circ}$ versus $45^{\circ}$. The results of these tests are shown in Figures 5.29 and 5.30 In both Figure 


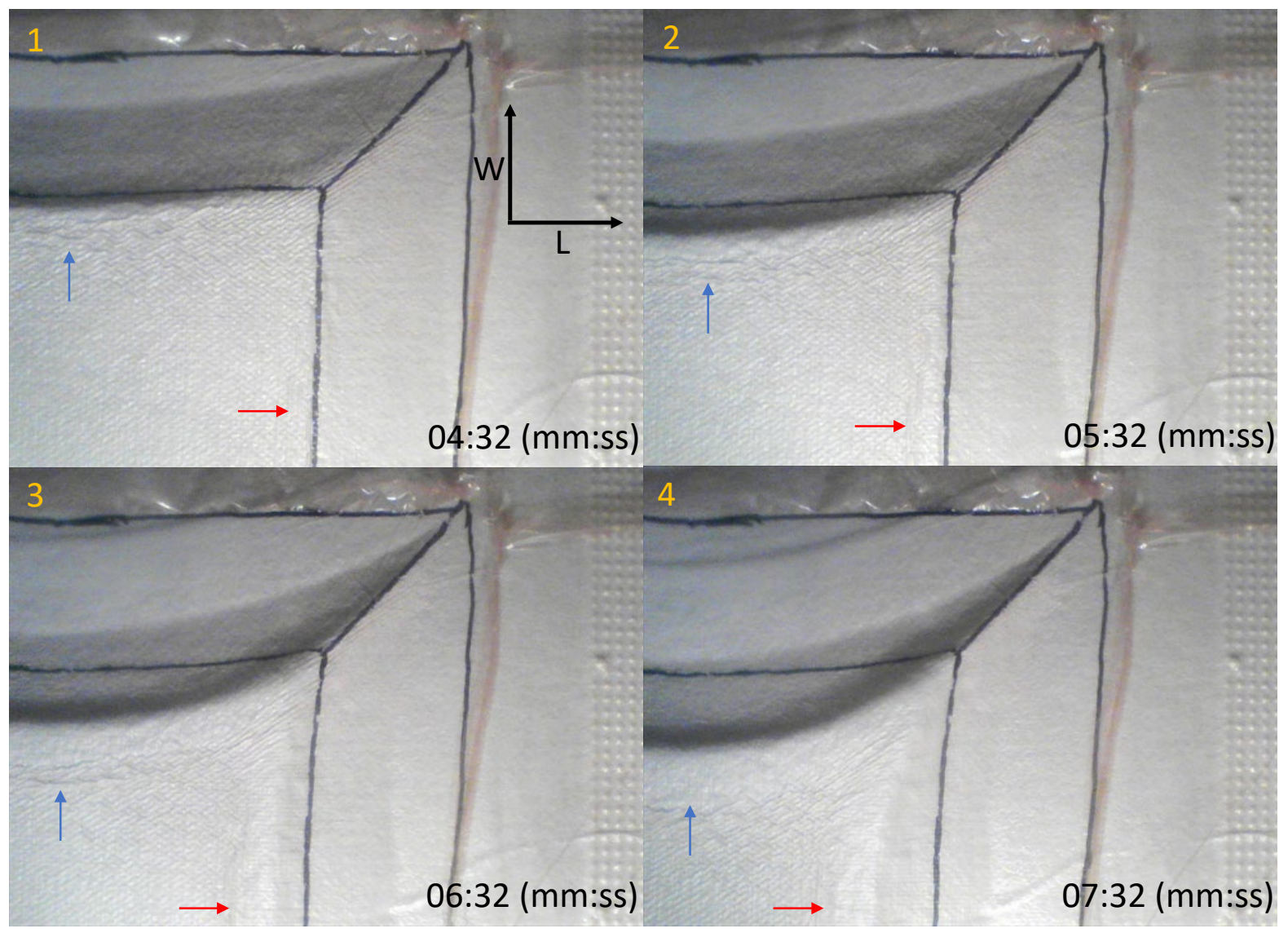

Figure 5.28: Crush pattern for $600 \mathrm{kPa}$ sample, displaying growth of the crush zone from image 1-4. W crush front (blue arrow) progresses at a faster rate than the L crush front (red arrow). Elapsed time from the application of temperature is displayed.

$5.29 \mathrm{~b}$ and Figure 5.30b the dark lines mark the initial position of the radius and chamfer bottom and serve as reference points for measuring displacement. The points corresponding to the camera snapshots are shown along the LVDT curves.

For the $20^{\circ}$ chamfer, initially, both the the top and bottom of the chamfer edge pull in at similar rates according to Figure 5.29b. The video footage confirms this observation as both the top (yellow arrow) and bottom of the chamfer (red arrow) move one square over an equivalent timeframe (photos 1 to 2 in Figure 5.29b). As crush continues, the bottom of the chamfer edge displaces at a faster rate than the chamfer radius. This is evidenced by the steeper slope of the bottom (cyan) LVDT, It is also apparent in the video footage, as the chamfer radius moves less than one square for each square that the bottom edge displaces (photos 2 to 3 ). However, this pattern is not constant. The top and bottom edge are again seen deforming at approximately the same rate of one square per unit time (photos 3 to 4 ). The same result is also seen in Figure 5.29a as the two LVDT curves display a similar average slope between points 3 and 4. Unfortunately, the top LVDT fails shortly after point 4, however video footage indicates that the chamfer edge continues to progress at a relatively constant rate of deformation along its length.

A similar pattern of deformation is seen at a chamfer angle of $45^{\circ}$, albeit at a much quicker rate. As 


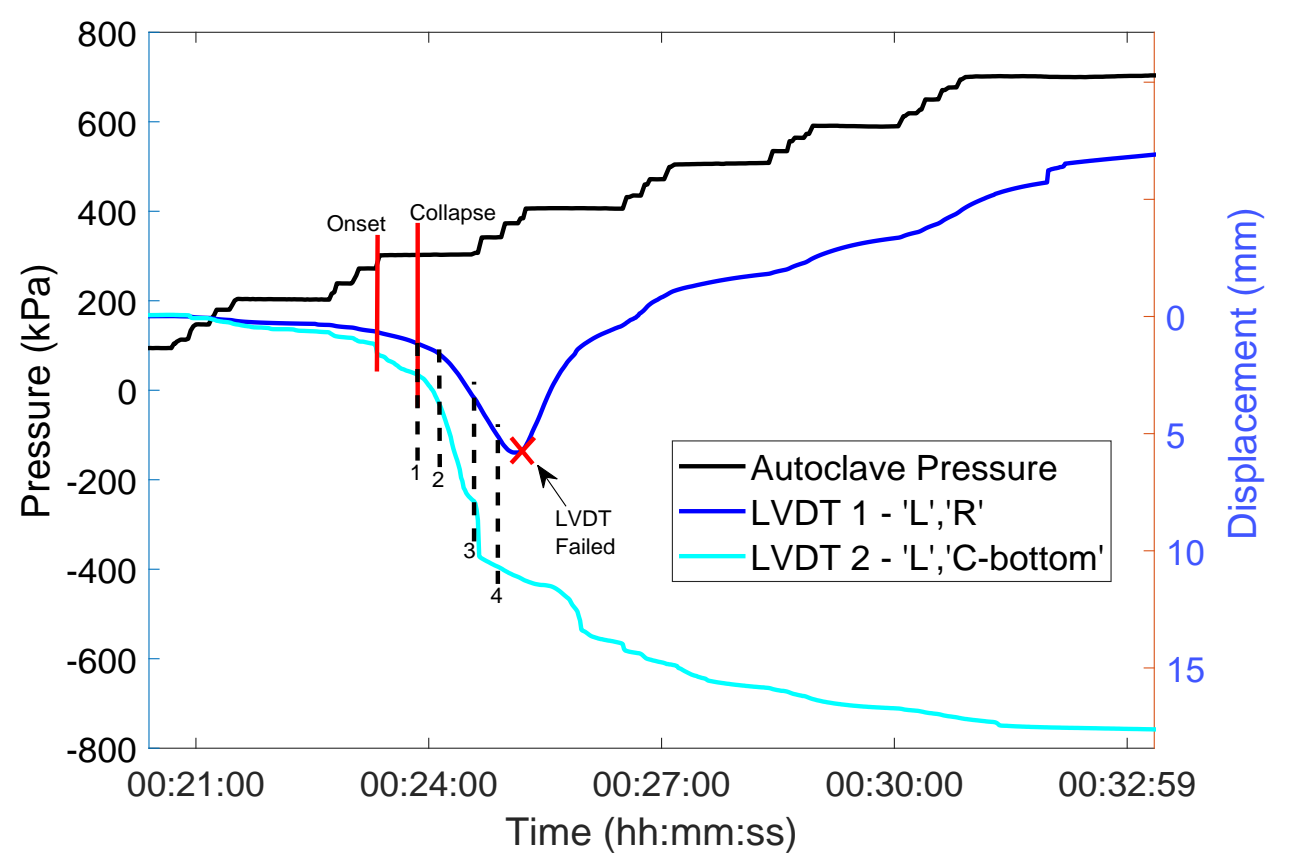

(a) In-situ LVDT data

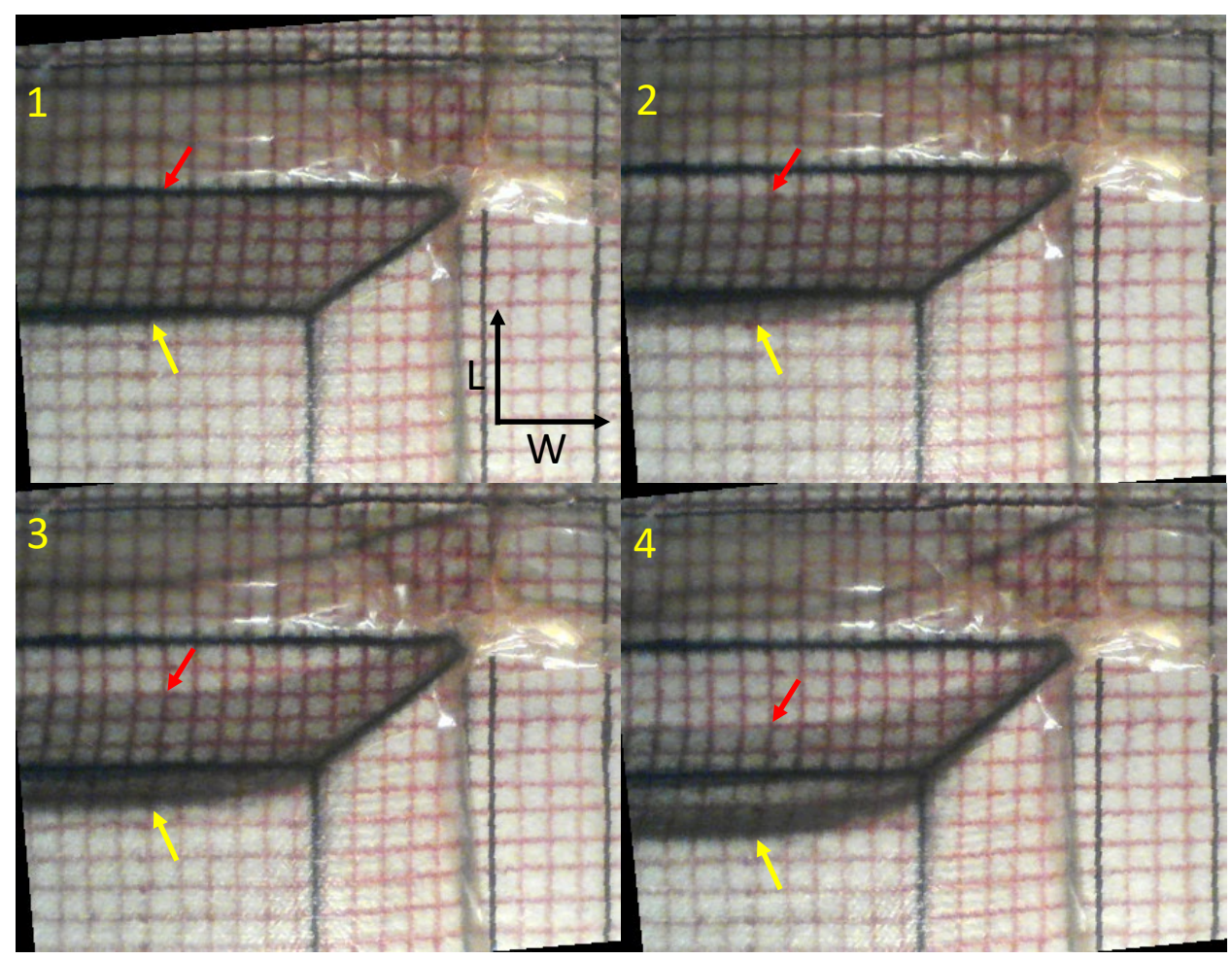

(b) In-situ video camera footage

Figure 5.29: Rate of deformation of the top and bottom of a $20^{\circ}$ chamfer edge witnessed through (a) in-situ sensors and (b) in-situ camera footage. Red arrows points to the bottom of the chamfer edge while yellow arrows points to the top. Each square in (b) is 12.7 x $12.7 \mathrm{~mm}$, allowing for the crush rate to be determined. 


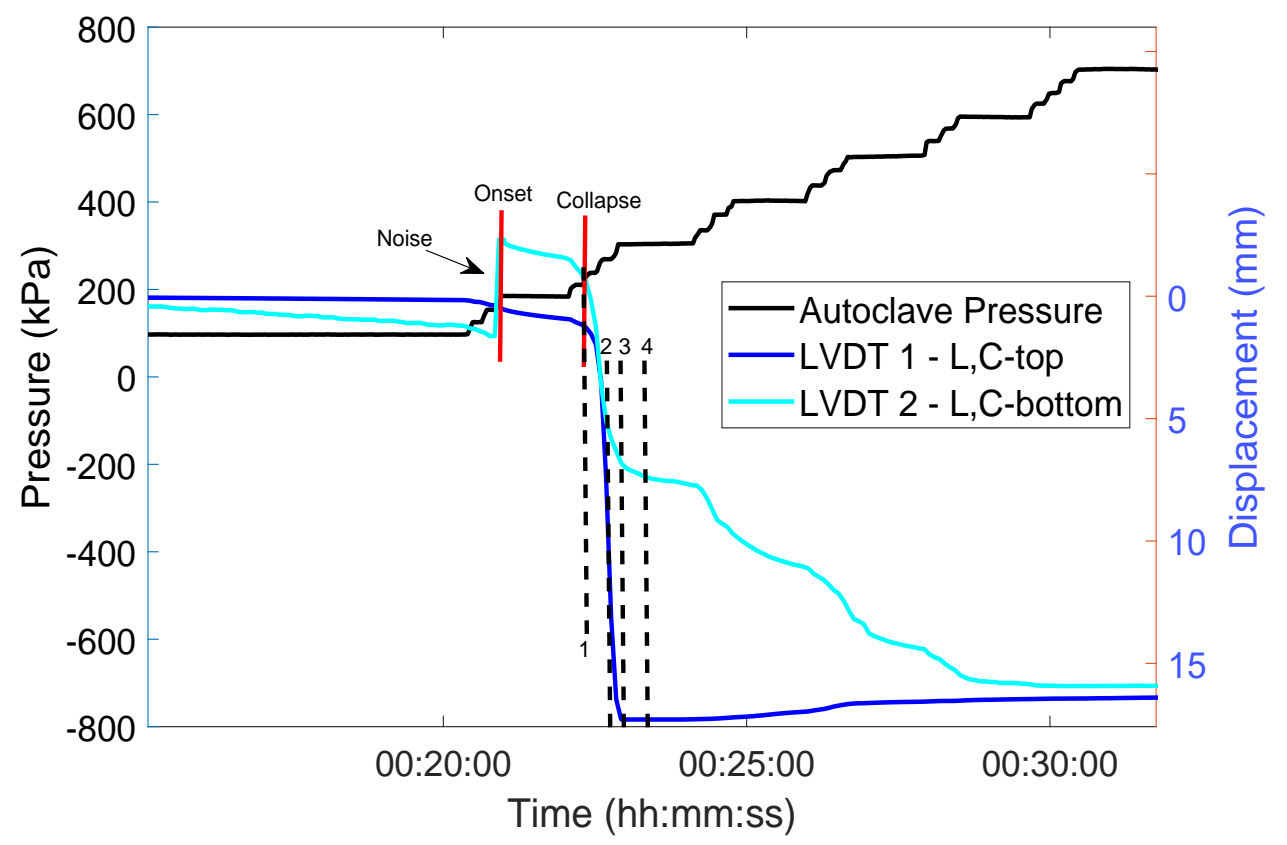

(a) In-situ LVDT data

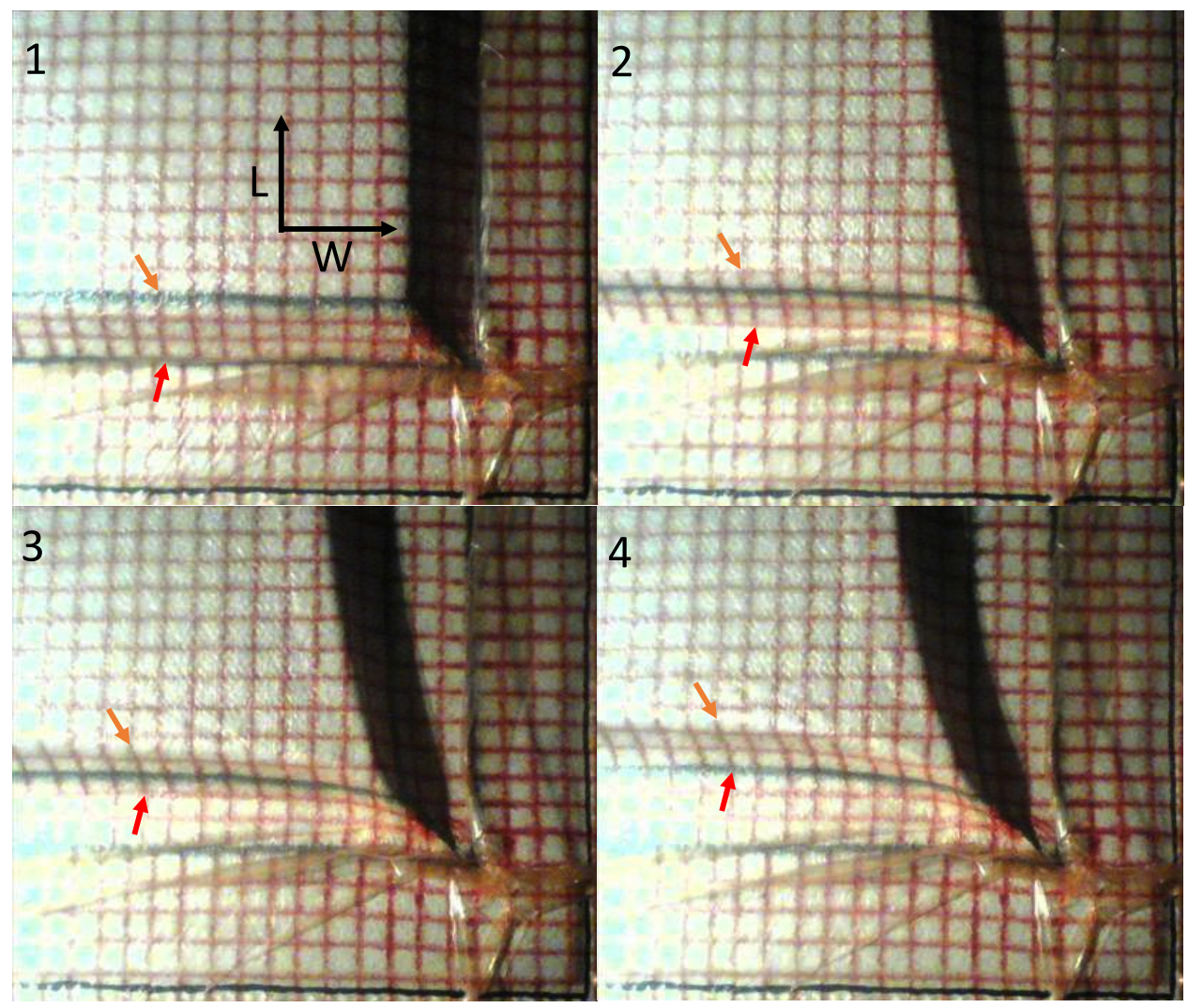

(b) [In-situ video camera footage

Figure 5.30: Rate of deformation of the top and bottom of a $45^{\circ}$ chamfer edge witnessed through (a) in-situ sensors and (b) in-situ camera footage. Red arrows points to the bottom of the chamfer edge while orange arrows points to the top. Each square in (b) is $12.7 \times 12.7 \mathrm{~mm}$, allowing for the crush rate to be determined. 
seen in Figure 5.29a, the initial rate of deformation is equivalent between the chamfer radius and bottom of the chamfer. Unfortunately, the positioning of the LVDTs is such that the LVDT located on the chamfer radius is at a steeper angle than than the LVDT on the bottom of the chamfer. Therefore, for an equivalent displacement, the upper LVDT measures significantly more movement. Moreover, the core quickly pulls away from the upper LVDT whereas the lower LVDT maintains contact for longer. Hence, other than the initial pattern of movement after collapse, the LVDT data can not be used to measure the rate of deformation of the two edges for this sample. The video footage has poor contrast, but it can still be used to approximate the rate of deformation of the chamfer. The radius is marked by an orange arrow and is distinguishable as the point where the squares begin to lay flat. The same is true for the bottom of the chamfer edge, which is marked by a red arrow. Figure 5.30b shows that between photo 1 and 2, the bottom and top edge each displace approximately one square as evidenced by the number of squares above and below the dark lines. Between photos 2 and 3 the bottom edge displaces one square while the top edge displaces less than one square. Between photos 3 and 4 both edges displace one square. The pattern is such that both edges initially deform at the same rate; the bottom edge then begins to displace at a faster rate than the top edge, before both then start moving at the same rate again. This matches with the pattern of collapse seen for the $20^{\circ}$ chamfer.

This is in line with the aforementioned observation that crush originates at the chamfer radius and spreads inward. Assuming crush originates at the chamfer radius and propagates toward the center of the panel, it follows that, initially, the bottom chamfer edge matches the displacement of the top edge; the entire chamfer will pull inward as a unit. However, as crush extends down the chamfer edge, only the bottom of the chamfer edge will displace. In other words, the bottom edge will deform at a faster rate than the top. This results in a steeper chamfer angle within the crush zone. Figure 5.31 provides a schematic explaining this. The first two illustrations show the difference in displacement for crush occuring just beyond the radius versus crush occuring along the chamfer edge. The third illustration is representative of the crush pattern exhibited in the experiments. Originally, crush occurs at the radius, dragging both the bottom and top edge in. As the chamfer itself crushes, the bottom edge begins to displace at a faster rate than the radius creating a concave surface. Further crush proceeds at a similar rate among the top and bottom edges as cells beyond the radius continue to collapse. For a $20^{\circ}$ chamfer angle, crush does not extend fully down the chamfer edge, preserving the original angle in the lower portion of the chamfer. However, for a $45^{\circ}$ chamfer, crush extends along the entire chamfer edge. Therefore, the difference in velocity between the bottom and top edge is more drastic than for a $20^{\circ}$ chamfer. The amount of deformation the bottom edge must undergo to entirely crush the $45^{\circ}$ chamfer edge is less than for a $20^{\circ}$ chamfer, since the $45^{\circ}$ chamfer is shorter (both cores are the same height). Hence, for an equivalent relative deformation between bottom and top edge, the change in slope angle is more severe for a steeper core.

The magnitude of the velocity vectors is affected by the level of densification; therefore, as the crush front progresses, the rate of crush decreases. In contrast, as pressure increases, deformation velocity increases. As such, the rate of core movement changes throughout the cure cycle and depends whether the part failed on a pressure hold or a ramp. To reduce complications associated with acceleration due 


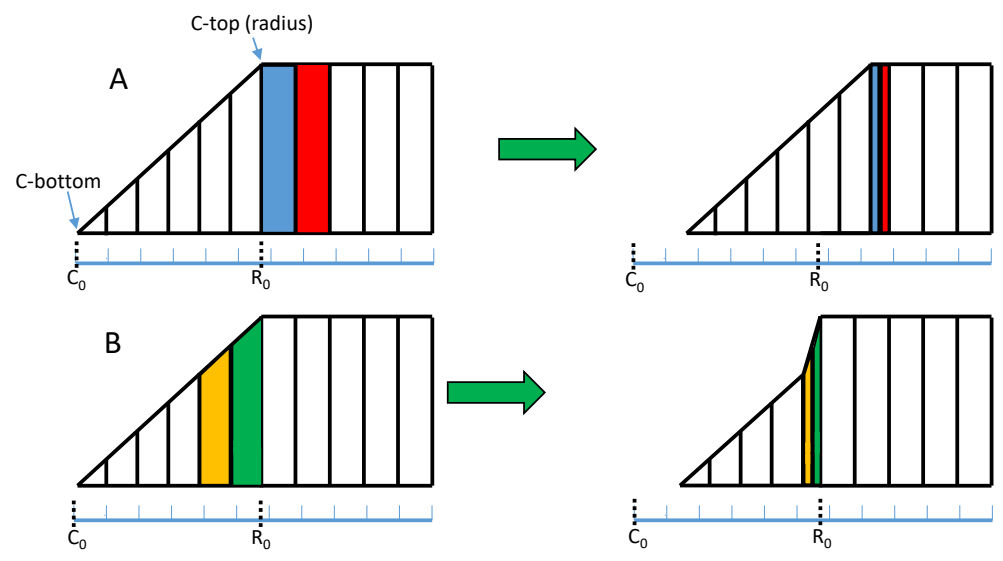

C
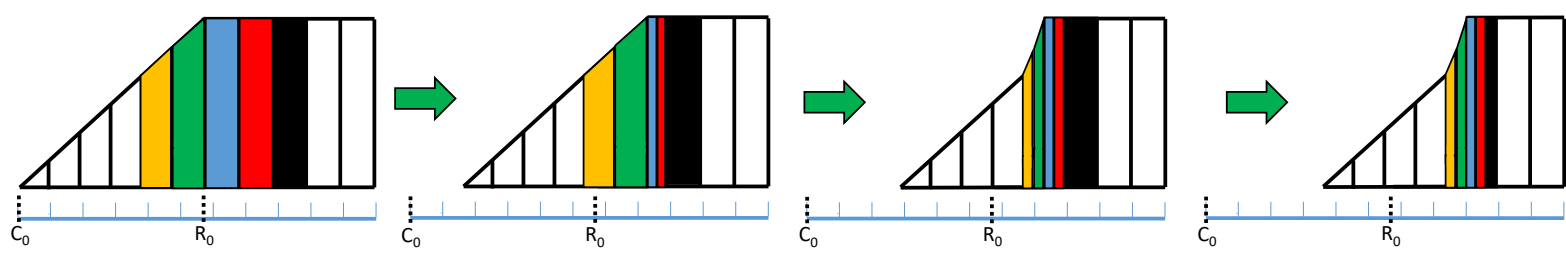

Figure 5.31: Schematic showing the manner of crush progression through the core. A: Crush initiates at chamfer radius and progresses inward - bottom and top of chamfer move at same rate. B: Crush initiates at chamfer radius and progresses down chamfer edge - bottom of chamfer displaces quicker than top creating a concave surface. C: Actual manner of crush progression through core - combination of A-B-A. 1 - Crush initiates at chamfer radius and progresses inward (similar to A). 2 - Chamfer edge crushes quicker than cells beyond radius (similar to B). 3 - Crush switches again to collapse cells further within core (similar to A).

to densification and increasing pressure, an instantaneous rate of core movement can be approximated according to the time it takes the chamfer to displace $12.7 \mathrm{~mm}$ (i.e. one square). While not a true instantaneous velocity, this measurement provides an estimate as to the relative rate of collapse for the two types of panels. For a chamfer angle of $20^{\circ}$ the rate of movement was determined to be $2.8 \mathrm{~mm} / \mathrm{s}$, whereas for the $45^{\circ}$ panel the rate of movement was $3.4 \mathrm{~mm} / \mathrm{s}$.

Intuitively it makes sense that collapse originates just beyond the chamfer radius. At this location the core is at maximum height, hence the effective area over which the horizontal component of pressure acts is also maximum. So too then is the applied force. Renn et al [42] also noted this. It therefore, follows that this would be the first location of cell collapse. As the collapsed cells increase in density, the force required to collapse cells farther ahead of the crush front would increase. Thus, the location of collapse shifts to along the chamfer edge. As densification increases along the chamfer edge, collapse occurs further within the core again and vice versa. The steeper the chamfer angle, the easier it is to crush cells along the chamfer edge. 

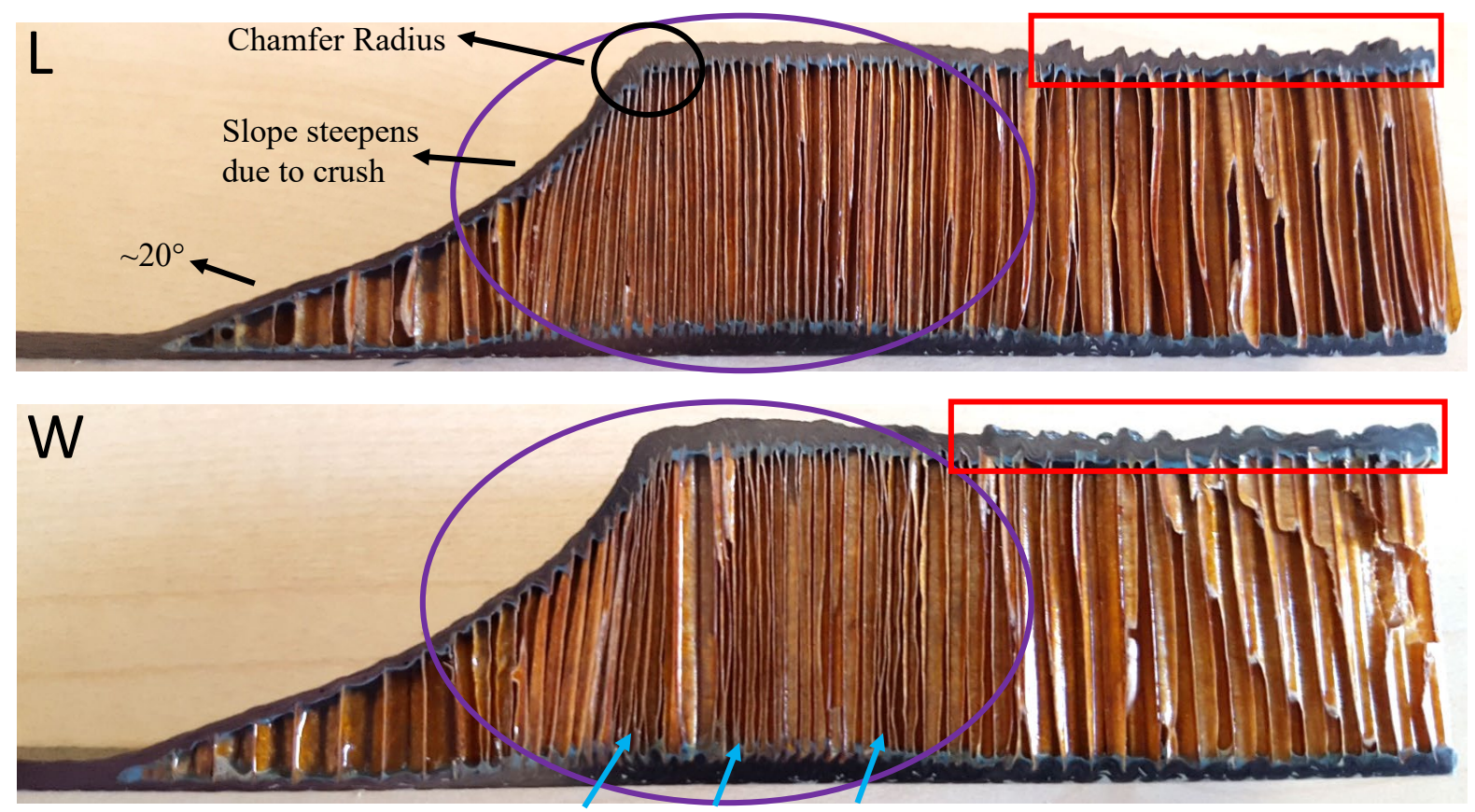

(a) Standard
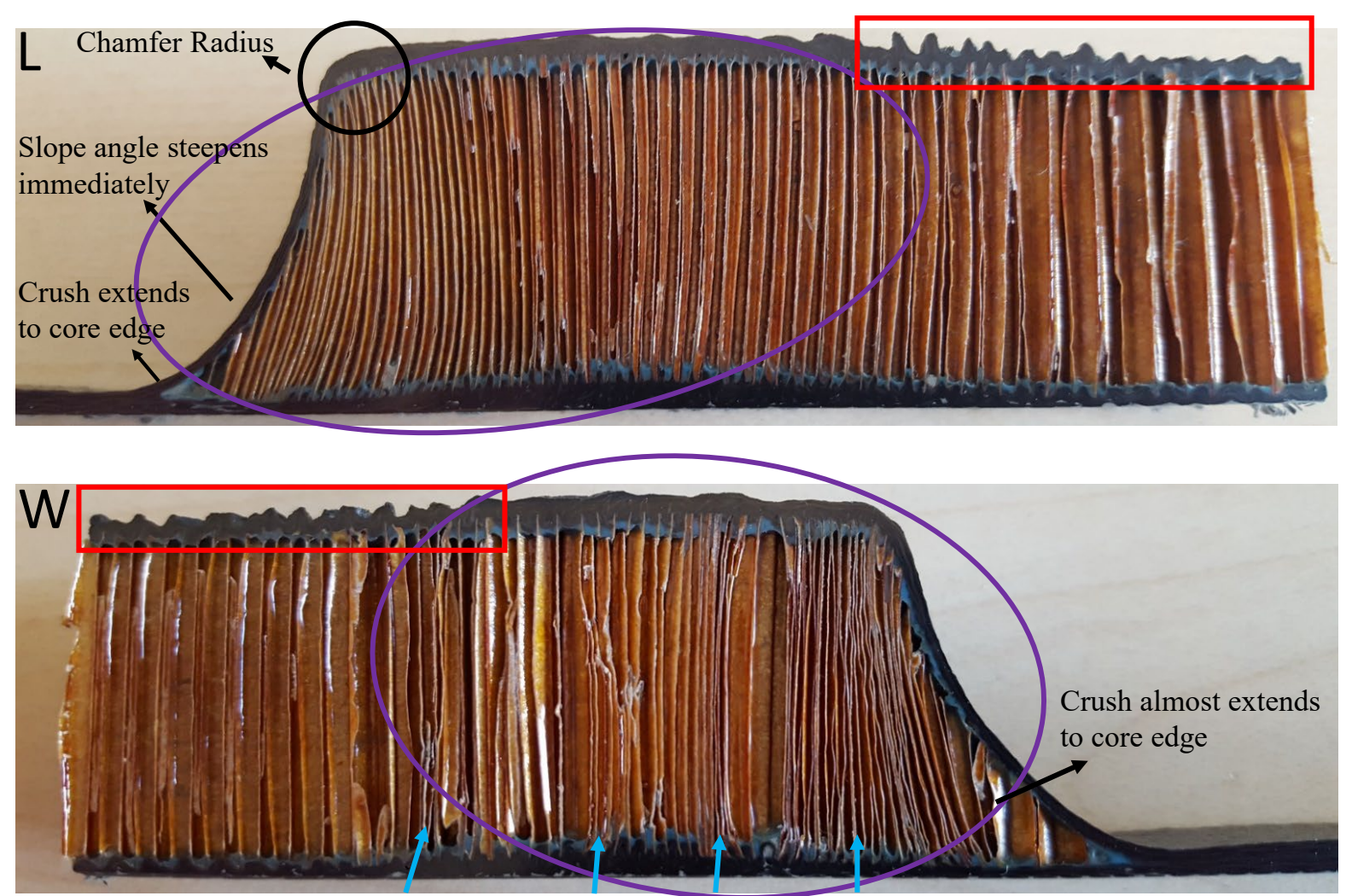

(b) $45^{\circ}$ chamfer

Figure 5.32: Through-thickness section cuts of processed samples. Purple indicates the crush zone, blue the densified regions, and red the bagside wrinkles. Additional features are outlined in black. (a) The $120^{\circ} \mathrm{C}$, standard layup and (b) 45 chamfer samples are provided here. Other samples are shown in the following images. 

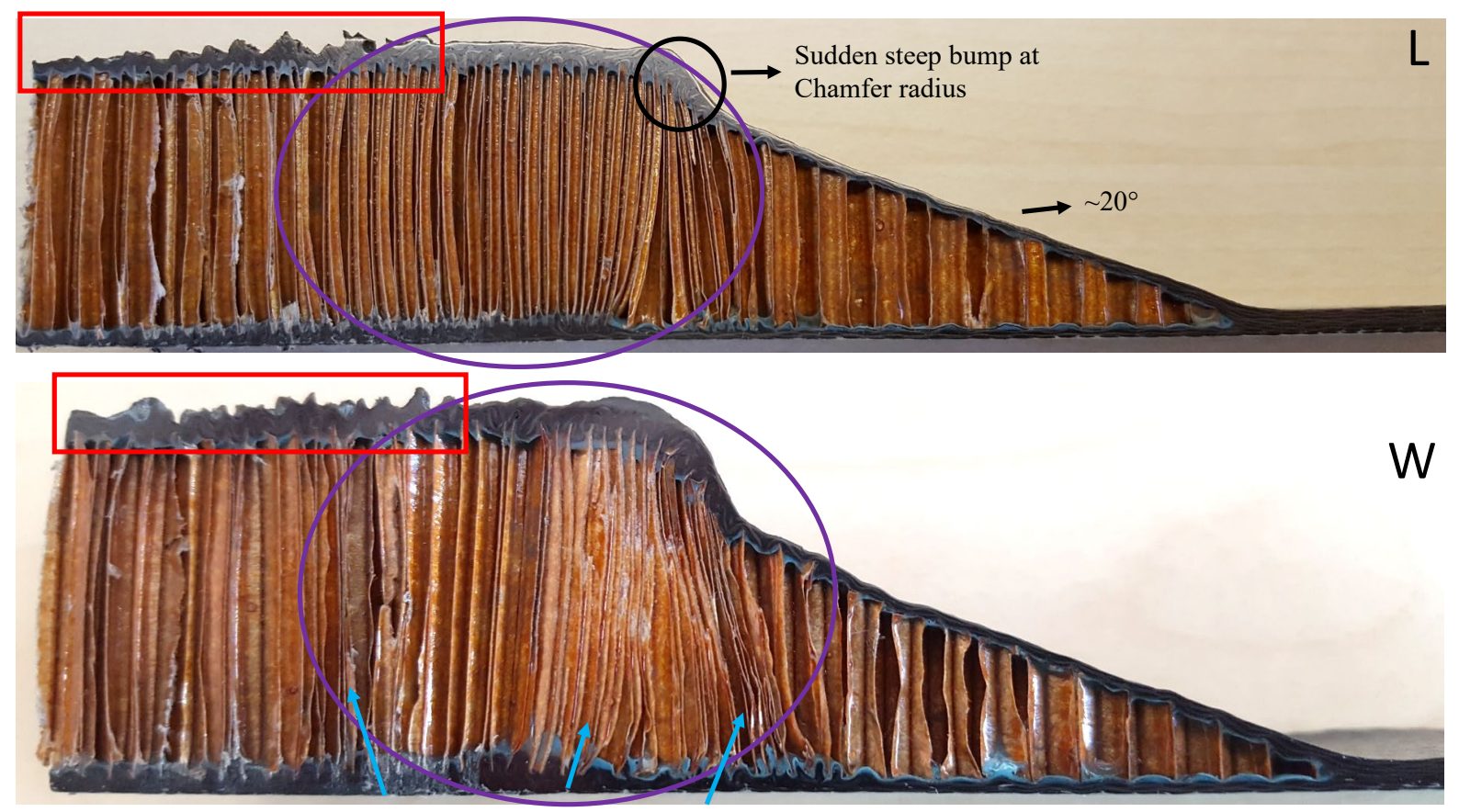

(a) CMS

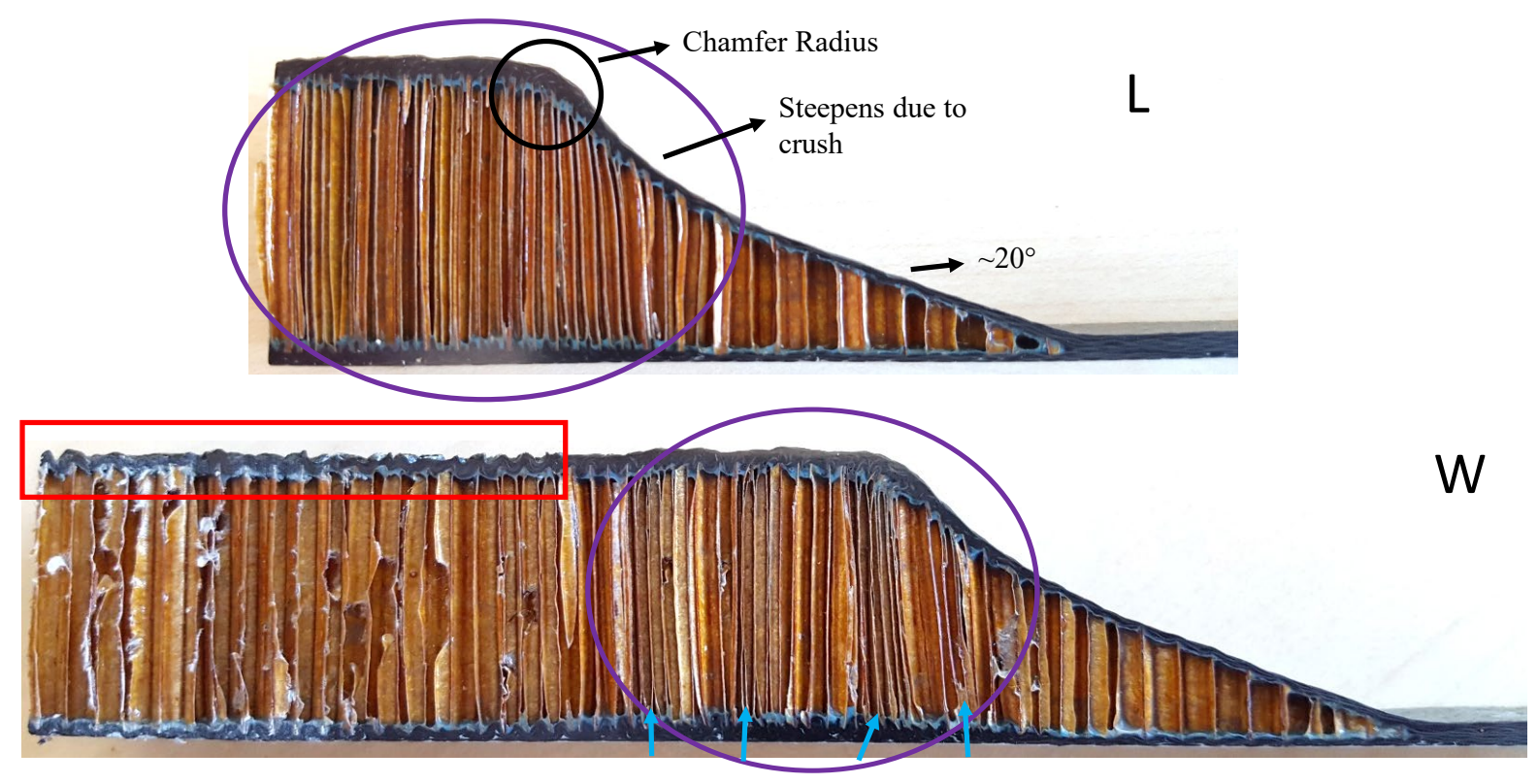

(b) Half tie-downs

Figure 5.33: (a) CMS and (b) half tie-down samples. 

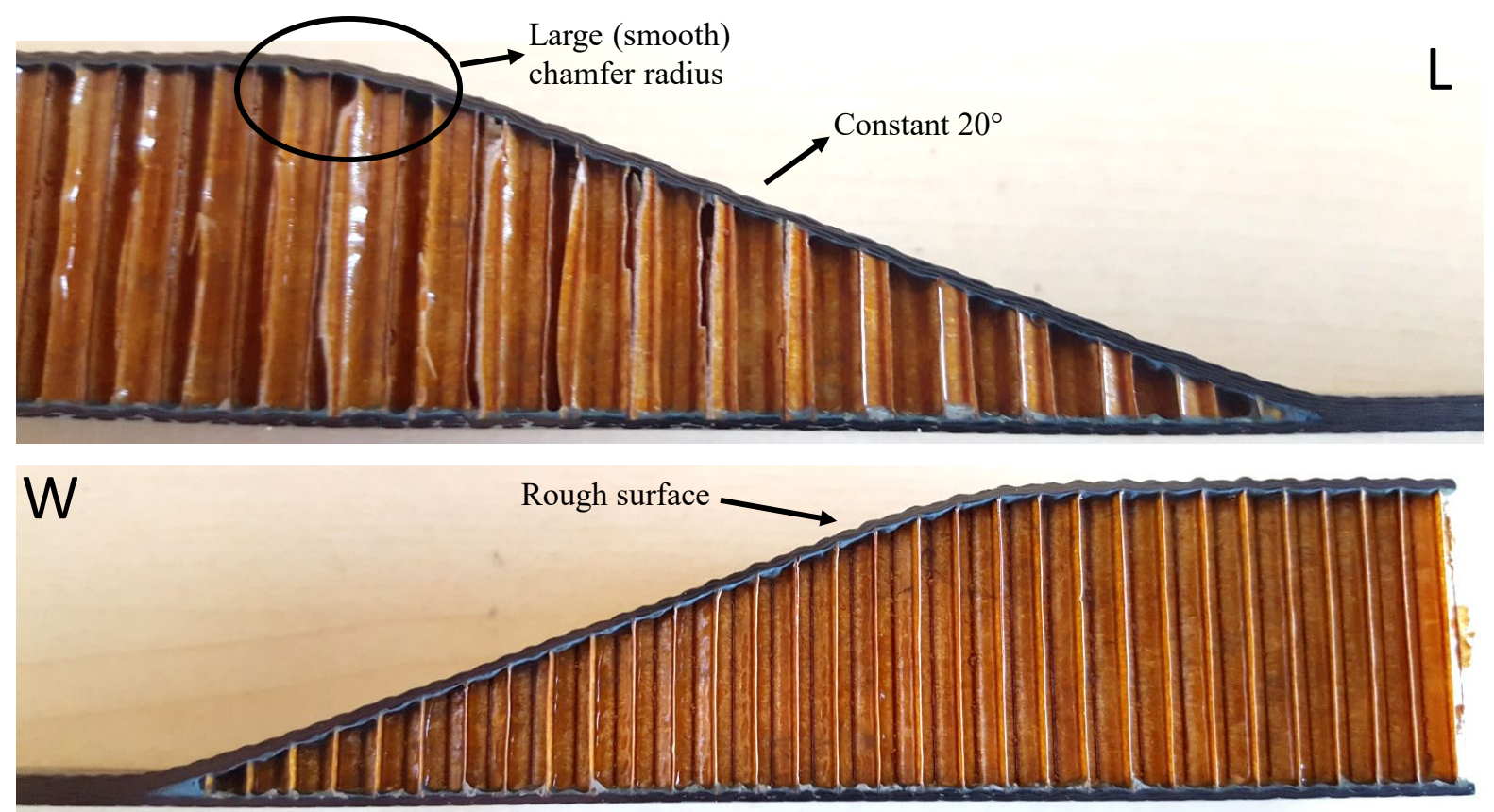

(a) Full tie-downs

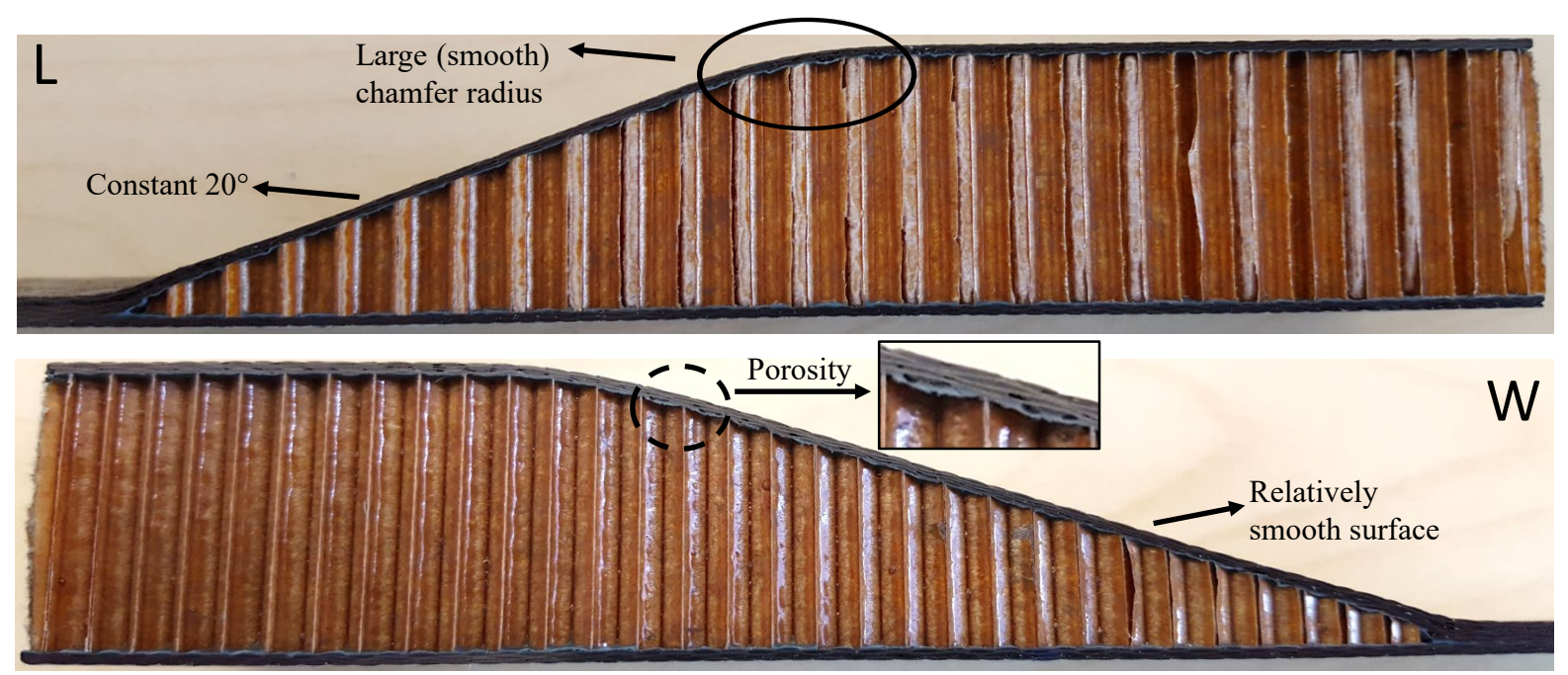

(b) Room temperature

Figure 5.34: Full tie-down and room temperature samples. 


\section{Through-thickness section cuts}

The specific patterns of crush between the $\mathrm{L}$ and $\mathrm{W}$ directions are highlighted in through-thickness cross sections, as shown in Figures 5.32 to 5.34. Samples from Section 5.3 are provided to represent the patterns observed during core movement, as changing the layup can alter the mechanics of the response. Equivalent images for the experiments outlined in Section 5.1 and Section 5.2 are given in Appendix B Generally speaking, the crush pattern for these latter samples show similar results to that of the $120^{\circ} \mathrm{C}$, standard layup test, with the primary difference being the size of crush zone.

The crush zone is defined as the area where beyond which densification decreases drastically to the point of not having collapsed. In the L direction, densification in the crush zone varies linearly, with the level of densification decreasing away from the chamfer radius. In the $\mathrm{W}$ direction, however, several unique highly densified regions are seen within the crush zone. Typically those nearest the radius exhibit the greatest densification. Between each densified region, the level of densification is reduced, indicating those rows have not fully collapsed. This pattern of crush fits with the propagation behaviour observed in the video footage and matches the expected collapse mode associated with the two directions. That is, homogeneous collapse in the L direction produces a uniform crush zone with a densification gradient that decreases in magnitude away from the initiation point. Row-by-row collapse in the $\mathrm{W}$ direction produces a crush zone with scattered regions of high densification throughout. It follows that if crush originates at the radius, densification should be highest at this location, as observed.

The specific pattern of core movement associated with each of the samples in Figures 5.32 to 5.34 , match with the results seen in Figure 5.26. Both the standard layup (Figure 5.32a) and the half tie down experiment (Figure 5.33b) display a uniform chamfer angle away from the radius. Closer to the chamfer radius the chamfer angle begins to steepen as you enter the crush zone. With a pre-processing chamfer angle of $45^{\circ}$ (Figure 5.32b), however, the crush zone extends to the core edge in the L direction and near the core edge in the $\mathrm{W}$ direction. As a result, the chamfer angle immediately begins to increase at the edgeband transition. Conversely, as previously mentioned, CMS (Figure 5.33a) virtually eliminates crush from occurring along the chamfer edge. The crush zone does, however, extend to the radius resulting in a steep bump at the chamfer radius. The full tie down experiment (Figure 5.34a) does not display visible core movement. However, as noted in Section 5.5.1, the surface is notably rougher than the standard layup sample processed at room temperature (Figure 5.34b) due to minor bending of the cell walls. In contrast, the room temperature sample experienced no core movement whatsoever, but displayed a large amount of porosity. In both cases, the chamfer edge remains at a constant angle equivalent to the pre-processing angle of the panels. The chamfer radii are also much larger as compared with the crushed samples, providing a smooth transition to the flat, bagside section of the panels.

Note that a smaller cut was made for the half tie down sample in the $\mathrm{L}$ direction. The cut does not display the area of core beyond the crush zone; hence, why no bagside wrinkles are seen in $5.33 \mathrm{~b}$

Figure 5.35 is a through thickness cut of the $120^{\circ} \mathrm{C}$, standard layup experiment. The cut is made along the entire length of the panel in the $\mathrm{W}$ direction such that the crush zone on either side of the panel is evident. The figure provides a visual for the general pattern of core movement as the crush front progresses through the panel from opposite sides. The pattern of movement is similar in the $\mathrm{L}$ direction 


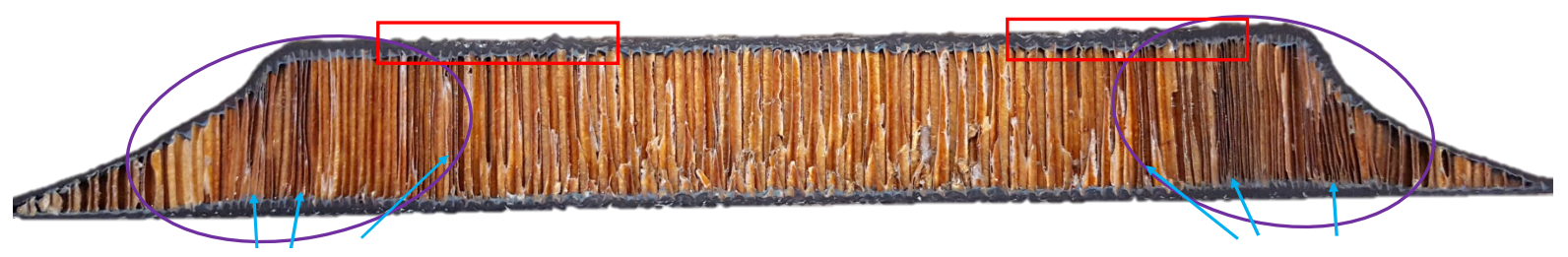

Figure 5.35: General crush pattern through panel in the $\mathrm{W}$ direction. Image is taken from the $120^{\circ} \mathrm{C}$, standard layup experiment. Purple indicates the crush zone, red the bagside wrinkles, and blue the densified regions.

Table 5.5: Average panel height within the crush zone compared with the degree of core movement in the $\mathrm{L}$ direction.

\begin{tabular}{|c|c|c|}
\hline Test & Height in L (mm) & Displacement in L (mm) \\
\hline Standard, room temp & 27.0 & 0 \\
\hline Standard, $95^{\circ} \mathrm{C}$ & 30.2 & 108 \\
\hline Standard, $120^{\circ} \mathrm{C}$ & 29.1 & 100 \\
\hline Standard, $180^{\circ} \mathrm{C}$ & $28.1^{*}$ & 41 \\
\hline Standard, $325 \mathrm{kPa}$ & 29.3 & 78 \\
\hline Standard, $600 \mathrm{kPa}$ & 29.2 & 85 \\
\hline $45^{\circ}$ chamfer & 29.0 & 128 \\
\hline CMS & 28.7 & 88 \\
\hline Half tie-down & 28.8 & 109 \\
\hline Full tie-down & 27.1 & 0 \\
\hline
\end{tabular}

without the intermittent, highly densified regions as shown in Figures 5.32 to $5.34 \mathrm{a}$. The cut was not made along the same line as that displayed in Figure 5.32a, although both sections are from the same sample.

In each case, immediately beyond the crush zone, large bagside wrinkles are seen. The wrinkles are a result of plies pulling in with the core and then buckling under compression to absorb the extra length that is generated. Within the crush zone, the plies nearest the core are compressed with the collapsed honeycomb and buckle with the cells themselves. Figure 5.36 shows a magnified image of this for the $45^{\circ}$ sample. Similar buckling is seen on the toolside surface of the crush zone. This indicates that upon collapse, instead of cellular compression occurring independent of plies, the immediate core-side plies buckle with the core. The plies buckle over the span of the underlying honeycomb cells. In other words, the section of ply over the honeycomb cell acts as an unsupported Euler column with a length equal to the honeycomb cell size. Given that plies nearest the core experience the greatest movement under standard processing conditions, the core-side plies likely move relative to other plies at first.

As the crush front progresses, bagside wrinkles coalesce together, leaving the crush zone relatively smooth. As a result, the panel height along this zone is markedly higher as compared with an undeformed panel. Figure 5.37 shows the comparison in height between an undeformed and severely deformed sample. The images were generated using data from CMM scans, allowing height at any given point to be extracted. The white bars indicate the crush zone in the $\mathrm{L}$ and $\mathrm{W}$ directions. Interestingly, a 


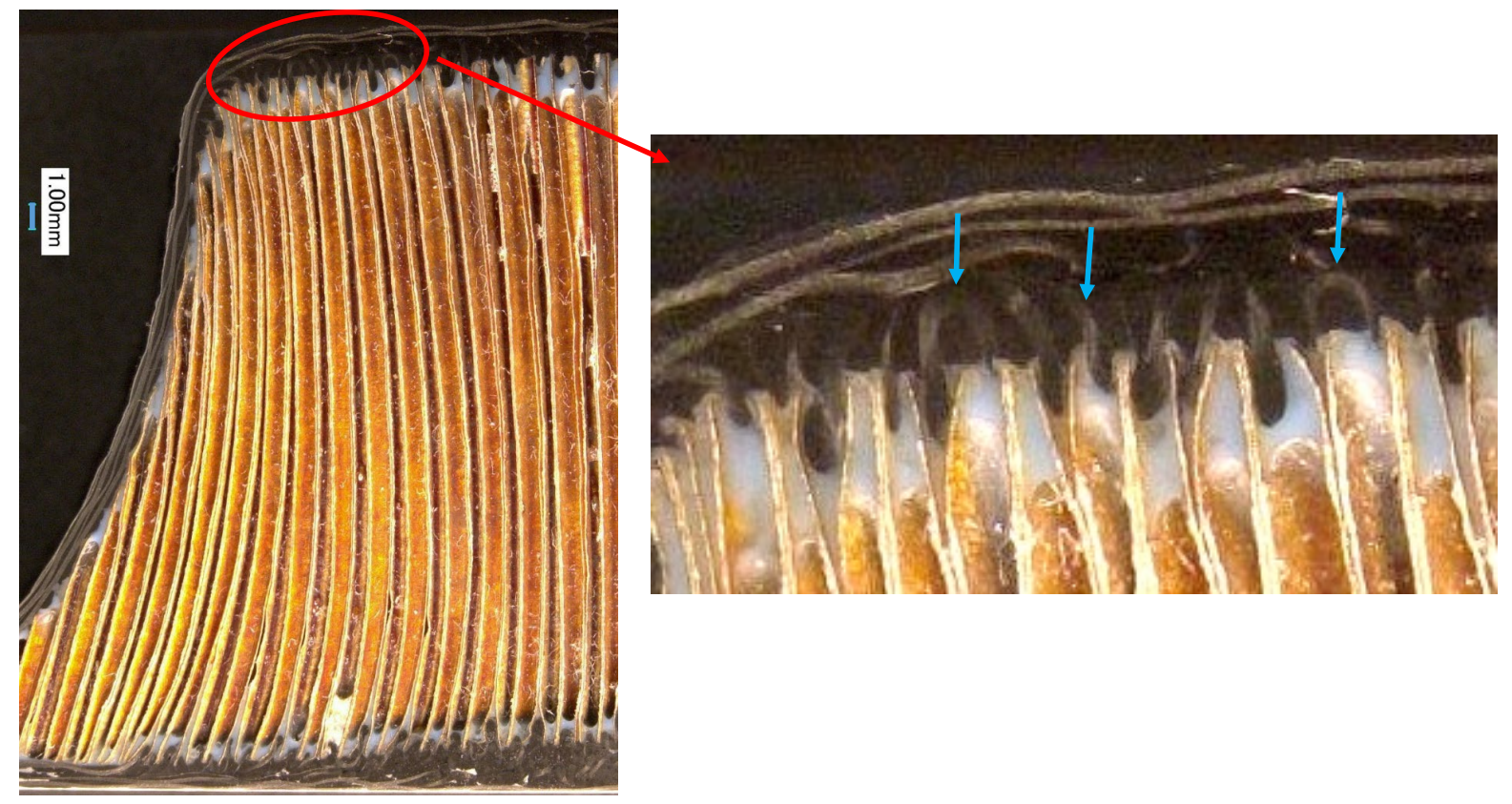

(a) Bagside ply buckling - taken from the $45^{\circ}-\mathrm{W}$ sample

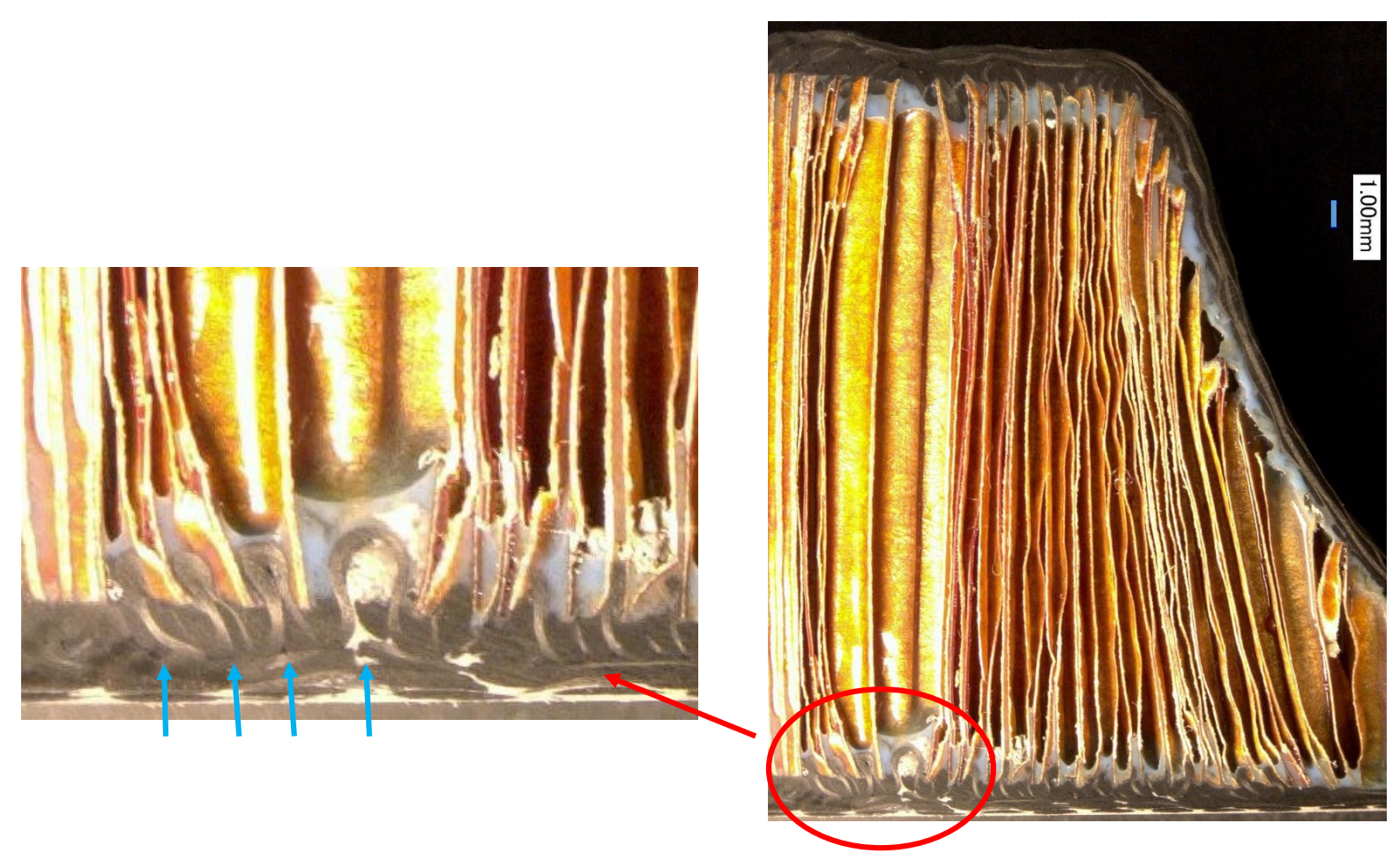

(b) Toolside ply buckling - taken from the $45^{\circ}-\mathrm{L}$ sample

Figure 5.36: Buckling of plies nearest the core (blue arrows) on the (a) bagside and (b) toolside surfaces within the crush zone. 


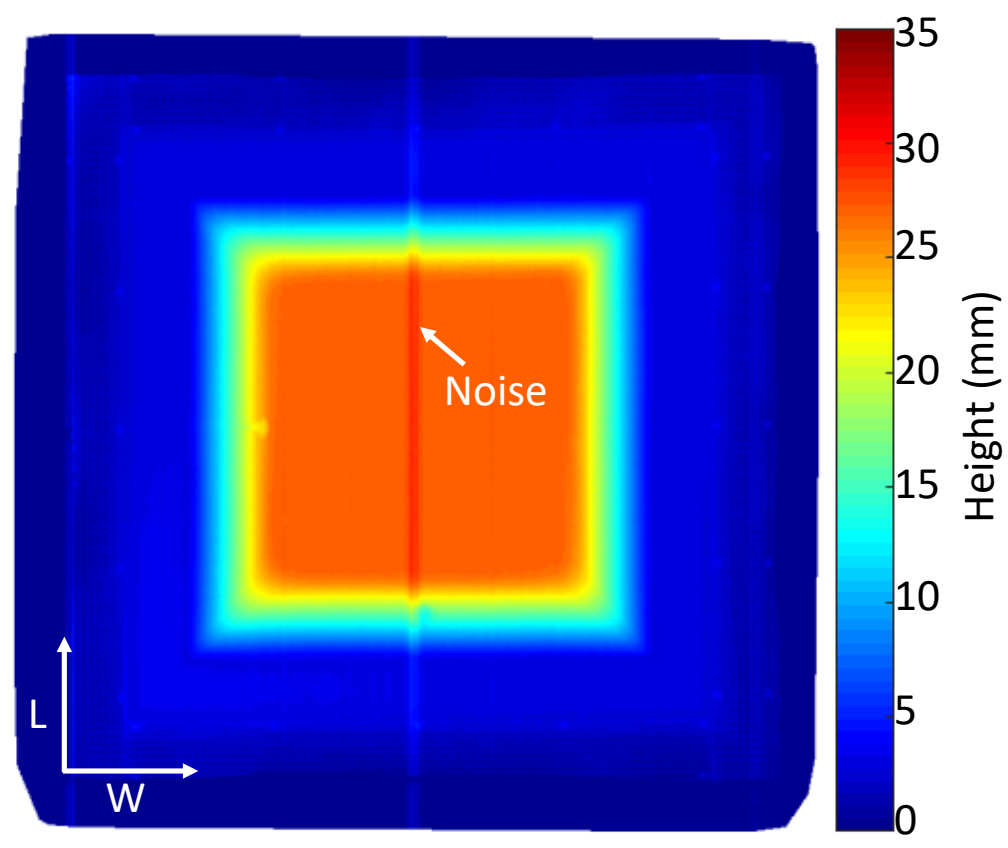

(a) No core movement - Full tie downs

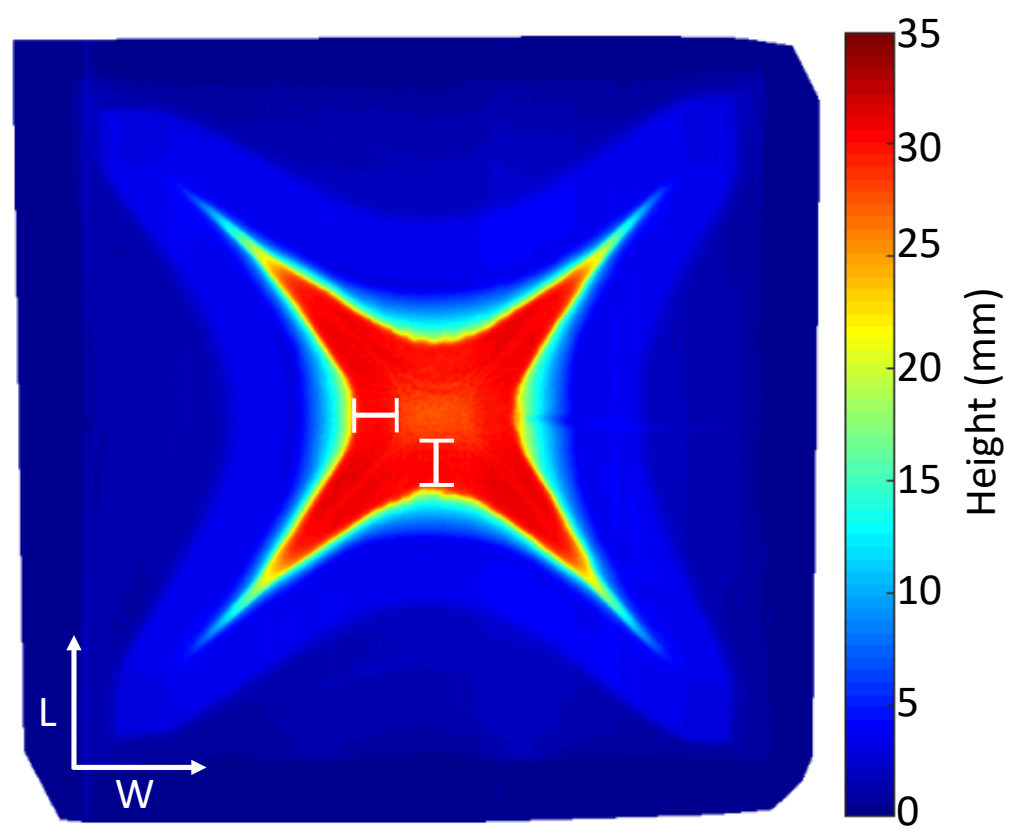

(b) Drastic core movement - Standard, $95^{\circ} \mathrm{C}$

Figure 5.37: Map of panel height extracted from CMM data for (a) no core movement and (b) drastic core movement. White bars in (b) represent the length of the crush zone in L and W. There is a notable increase in height along the crush zone. 
Table 5.6: Average panel height within the crush zone compared with the degree of core movement in the $\mathrm{W}$ direction.

\begin{tabular}{|c|c|c|}
\hline Test & Height in W $(\mathbf{m m})$ & Displacement in W (mm) \\
\hline Standard, room temp & 27.0 & 0 \\
\hline Standard, $95^{\circ} \mathrm{C}$ & 29.9 & 104 \\
\hline Standard, $120^{\circ} \mathrm{C}$ & 29.7 & 86 \\
\hline Standard, $180^{\circ} \mathrm{C}$ & $28.1^{*}$ & 16 \\
\hline Standard, $325 \mathrm{kPa}$ & 28.8 & 52 \\
\hline Standard, $600 \mathrm{kPa}$ & 29.6 & 94 \\
\hline $45^{\circ}$ chamfer & 28.8 & 124 \\
\hline CMS & 28.6 & 82 \\
\hline Half tie-down & 28.7 & 99 \\
\hline Full tie-down & 27.1 & 0 \\
\hline
\end{tabular}

greater degree of core movement does not necessarily result in a higher average panel height throughout the crush zone. This is shown in Tables 5.5 and 5.6. A possible explanation for this is that it is not the extent of crush that matters, but rather the level of densification within the crush zone. Assuming the section of ply over each honeycomb cell buckles with collapse of the cell, then the level of buckling, and therefore height, will correspond to the degree of densification of that cell. As wrinkles coalesce, the average height within the crush zone will correspond to the average level of densification. At high levels of densification, the average increase in height of the first bagside ply within the crush zone should approach half the honeycomb cell size. As additional plies buckle, the total increase in height of the panel may be even greater. In these experiments, the cell size was $3.175 \mathrm{~mm}$, with the average height of undeformed panels around $27 \mathrm{~mm}$. This gives a minimum average height of approximately $28.6 \mathrm{~mm}$ within the crush zone for panels exhibiting core movement. Most of the crushed panels display a total height near or above this value. The $95^{\circ} \mathrm{C}$ test displays the greatest total height within the crush zone. This may be attributed to a greater degree of densification or simply be the result of inconsistencies in the amount of paint used during CMM scanning. Paint film thickness was not measured for the panels.

Note that because the $180^{\circ} \mathrm{C}$ test did not allow much time for progression of core movement, wrinkles within the crush zone did not yet coalesce. This is particularly true in the L direction. Hence, the height varies considerably between points, thus skewing the average height. In the $\mathrm{W}$ direction, few wrinkles were picked up. Therefore, the height presented in Table 5.6 is the max amplitude of an observed wrinkle. Values displayed in Tables 5.5 and 5.6 were taken along the center lines of the panels.

\section{In-plane section cuts}

The progression of core movement can also be observed from in-plane section cuts, similar to those presented in Figure 5.25. Figure 5.38 shows two different samples displaying drastic core movement. In each case, the sample has undergone densification. Figure $5.38 \mathrm{a}$ is from the $120^{\circ} \mathrm{C}$, standard layup sample and is representative of the typical pattern of deformation seen amongst all samples exhibiting core movement. Figure $5.38 \mathrm{~b}$ is from the $600 \mathrm{kPa}$ sample, and represents the only case where the $\mathrm{W}$ 


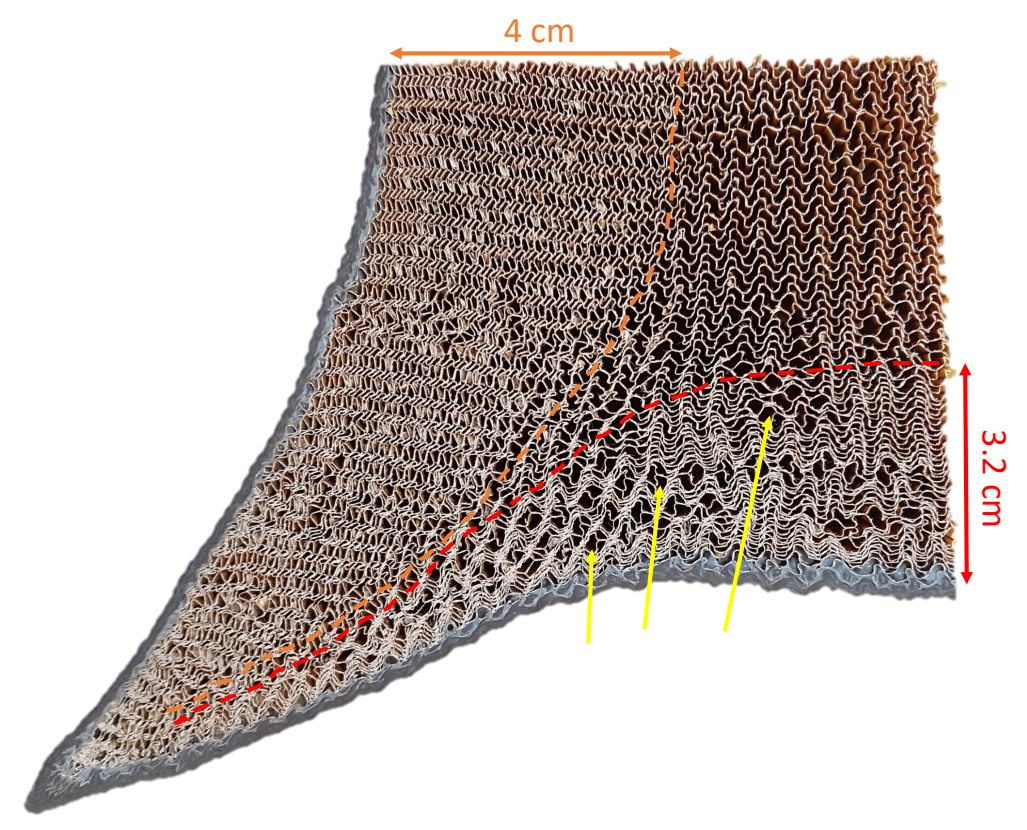

(a) $120^{\circ} \mathrm{C}$, standard layup

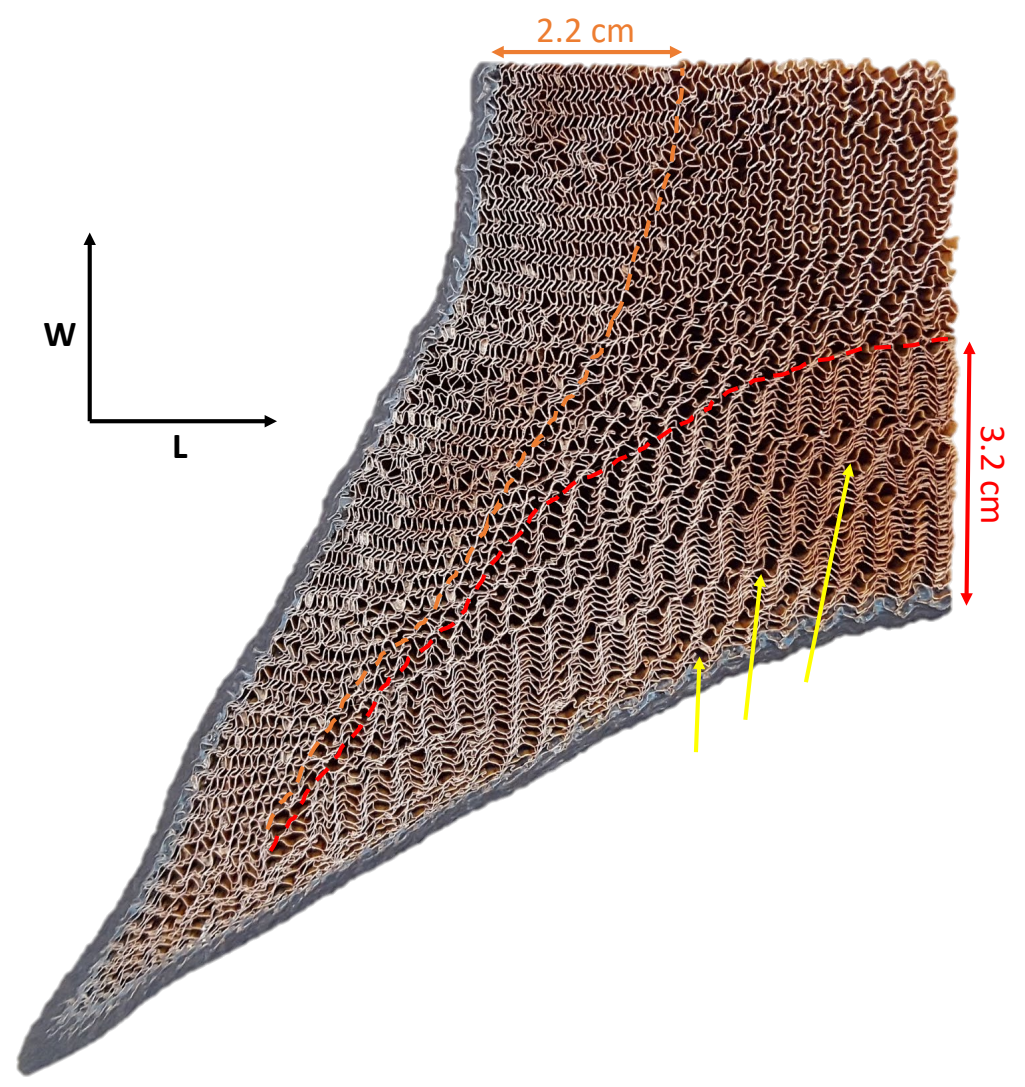

(b) $600 \mathrm{kPa}$, standard layup

Figure 5.38: In-plane section cuts of processed panels showing densification along the $\mathrm{L}$ and $\mathrm{W}$ directions. The orange line represents the $\mathrm{L}$ crush front whereas the red line represents the $\mathrm{W}$ crush front. Yellow arrows point to regions of low densification in the non-ribbon direction 
direction exhibited a higher degree of core movement than the L direction. In both images, the pattern of collapse in the $\mathrm{L}$ and $\mathrm{W}$ directions is apparent. Just as observed in the through-thickness sections, the L direction cells show fairly uniform densification within the crush zone. A slight gradient is observed, with the highest level of densification occuring at the core edge and decreasing towards the center of the panel. For the most part, however, the level of densification remains constant until a point, signifying the edge of the crush zone (i.e. the crush front), where after densification is significantly reduced. In the $\mathrm{W}$ direction, regions of high densification are intermittent with regions of less densification. This creates low density pockets within the crush zone that have not fully collapsed yet. The crush front is identified by the last region of high densification. Beyond this point, densification is reduced. Along their respective edges, the unique collapse pattern in the $\mathrm{L}$ and $\mathrm{W}$ directions is apparent. However, towards the center of the panel, the patterns interfere with one another creating a mixed response. This is common in biaxial compression of naked honeycomb (i.e. without facesheets) [22]. With the sandwich panels presented here, often one collapse pattern will dominate the other. Typically, this is the L collapse pattern.

In Figure 5.38a, densification in the $\mathrm{L}$ direction extends considerably further into the panel than in the $\mathrm{W}$ direction. This is true for all other panels except that shown in Figure 5.38b, where the crush zone is larger in the $\mathrm{W}$ direction. This implies that the two crush fronts vie for progression through the core. If one crush front progresses faster than the other, the faster collapse mode will constitute the majority of the densification pattern. The slower crush front will eventually be arrested by the pre-collapsed cells of the faster crush front. Given that in every test but one, the L direction exhibited greater core movement than the $\mathrm{W}$ direction, it can be concluded that, in general, homegenous buckling in L presents a faster collapse mode than row-by-row plastic deformation in $\mathrm{W}$. That said, any given row in $\mathrm{W}$ at its bending limit could collapse [23]. It is therefore possible that rows further in the core could collapse before rows nearer the radius. As a result the crush zone could be extended beyond that of the $\mathrm{L}$ crush zone, thus "winning" the biaxial response. Any un-collapsed cells within the crush zone are isolated from failure in $\mathrm{L}$ and may collapse later, thus still allowing for significant core movement in the $\mathrm{W}$ direction. This a possible explanation as to the failure pattern exhibited in the $600 \mathrm{kPa}$ sample, where $\mathrm{W}$ deformation was more extensive. In fact, several low density regions are seen within the sample's crush zone (Figure 5.38b). Moreover, the densified regions that are present are less dense as compared with equivalent regions in Figure 5.38a. This suggests an extended crush projection of the nature described above.

In the $120^{\circ} \mathrm{C}_{\text {orig }}$ and $180^{\circ} \mathrm{C}$ tests core movement progressed quicker in the $\mathrm{L}$ direction; likely due to a faster rate of collapse. However, as shown for the $95^{\circ} \mathrm{C}$ and $600 \mathrm{kPa}$ samples, the rate of progression of core movement does not necessarily correspond to the rate of progression of the crush front. Unfortunately, for many of the experiments one LVDT sensor failed. Thus, the rate of progression of core movement in the two directions is indeterminable for the other tests. Video footage in general does not provide clear enough contrast to accurately measure the rate of core movement (excluding the two tests where a grid pattern was implemented).

Additional in-plane section cuts are provided in Appendix B. 
Table 5.7: In-core pressure at onset and collapse.

\begin{tabular}{|c|c|c|}
\hline Test & Onset [sensor 1, sensor 2] (kPa) & Collapse [sensor 1, sensor 2] (kPa) \\
\hline Standard, $95^{\circ} \mathrm{C}$ & 77.1 & 74.1 \\
\hline Standard, $120^{\circ} \mathrm{C}$ & 114 & 113.5 \\
\hline Standard, $180^{\circ} \mathrm{C}$ & $116.4,66.3$ & $* 179.8,86.6$ \\
\hline Standard, $325 \mathrm{kPa}$ & $102.4,81.42\left(\right.$ at $\left.55^{\circ} \mathrm{C}\right)$ & $113.3,92.4\left(\right.$ at $\left.82^{\circ} \mathrm{C}\right)$ \\
\hline Standard, $600 \mathrm{kPa}$ & $102.5\left(\right.$ at $\left.34^{\circ} \mathrm{C}\right)$ & $97.1\left(\right.$ at $\left.43^{\circ} \mathrm{C}\right)$ \\
\hline $45^{\circ}$ chamfer, $120^{\circ} \mathrm{C}$ & $* 24.9$ & $* 59.8$ \\
\hline CMS, $120^{\circ} \mathrm{C}$ & Failed & Failed \\
\hline Half tie-down, $120^{\circ} \mathrm{C}$ & Failed & Failed \\
\hline
\end{tabular}

\subsubsection{In-core pressure}

During vacuum application within the autoclave, the in-core pressure sensors often showed slightly higher pressure than vacuum. Upon venting of the vacuum bag, the in-core pressure increased fairly rapidly to a level similar to that of the bag. This is shown in Figure 5.39. The magnitude of in-core pressure at onset and collapse for each sample displaying core movement is provided in Table 5.7. In most cases, onset and collapse occurred after venting, with an in-core pressure of around $1 \mathrm{~atm}$. The $45^{\circ}$ chamfer displays considerably lower in-core pressure since onset occurred prior to venting of the bag and collapse occurred immediately after venting; before the core pressure was able to stabilize (Figure 5.15). Interestingly, there is no significant change in in-core pressure between onset and collapse, as well as following collapse for most tests. It is expected that in-core pressure would increase as cells are compressed and volume is reduced. The fact that this was not seen indicates that gas is vented as the honeycomb compresses. It is possible that as cells fail, new air paths are created through which gas can flow easily. More likely, however, is that the pressure sensor wires provide a continual path for gas flow. In addition, a cut-line was made in the core around the wires in order to firmly seat the sensors. This too would have promoted gas exchange between cells. With this setup, pressure sensors cannot be used to track core movement on their own. Furthermore, in each case, the sensors failed as crush progressed. The pressure at collapse for the $180^{\circ} \mathrm{C}$ sample demonstrates the expected result of a pressure increase through cell volume reduction.

The results suggest that the contribution of in-core pressure to core movement is similar to the level of vacuum application. This matches results presented by Alteneder et al and Renn at al [6, 42]. Gas paths introduced by insertion of the sensor wires exaggerated this effect. Therefore, the measured incore pressure is not necessarily indicative of the in-core pressure further within the core (away from the sensors). Prior to venting of the bag, the samples displayed an in-core pressure of $10-20 \mathrm{kPa}$ on average. It is possible that the internal pressure further within the core remained closer to this range of pressure. This would exacerbate the force acting on the core. However, as the panels were never kept under active vacuum for extenuating periods of time, it is also possible that the center region of the core never achieved depressurization in the first place; thus, remaining near $1 \mathrm{~atm}$ throughout processing.

Under typical cure cycles, pressure and temperature are applied simultaneously. Once the autoclave pressure reaches $1 \mathrm{~atm}$ gauge, the vacuum bag is vented. If the consolidation pressure is less than 125 


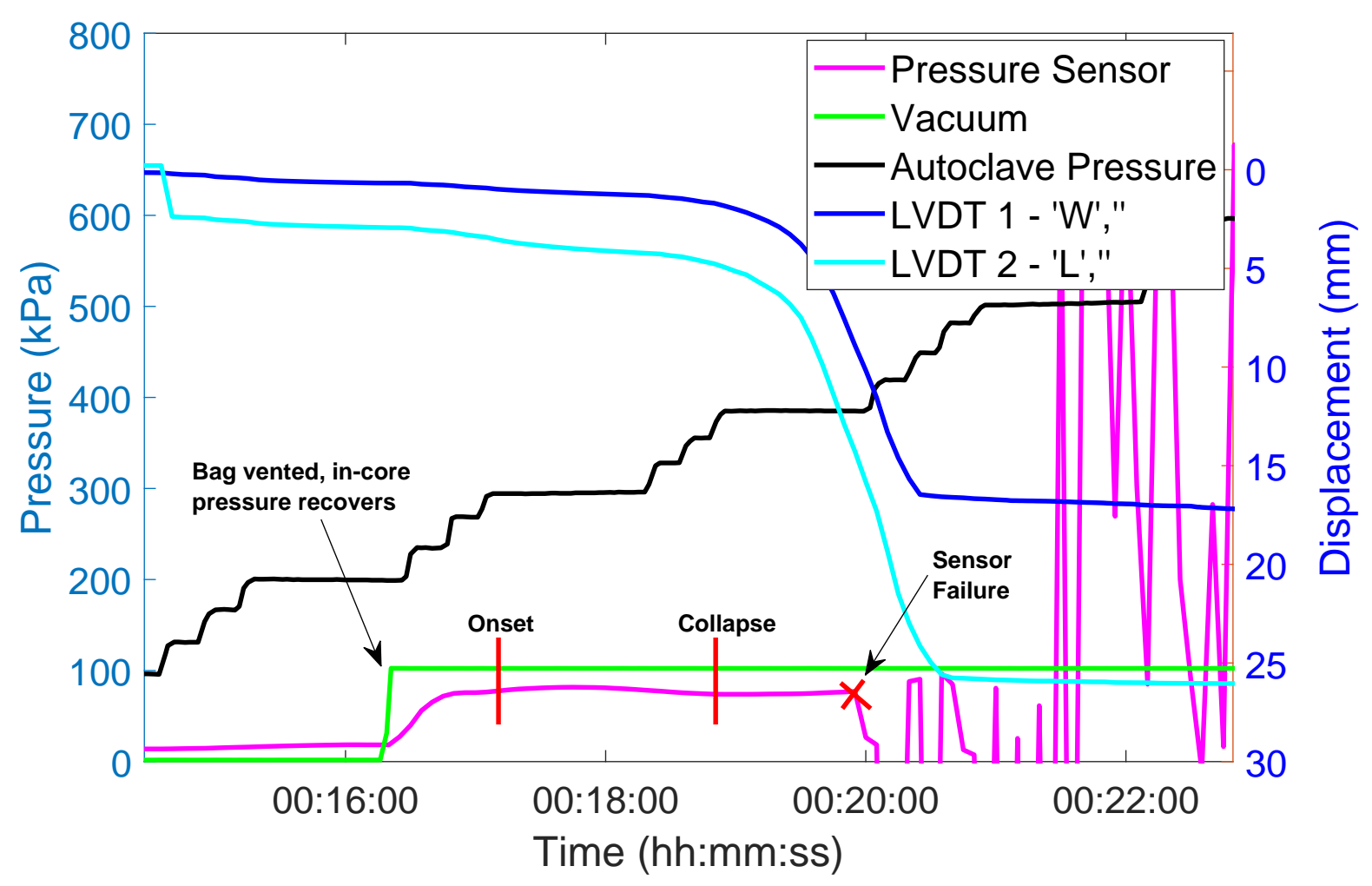

Figure 5.39: In-core pressure sensor response to core movement taken from the standard, $95^{\circ} \mathrm{C}$ sample. Pressure recovery is captured upon release of vacuum. No difference in in-core pressure is observed between onset and collapse. Following collapse, considerable noise is seen due to pulling of the sensor wires.

$\mathrm{kPa}$, the prepreg remains relatively porous allowing gas to flow in an out of the core according to the level of vacuum bag pressure [6]. As consolidation pressure is increased, gas becomes entrapped within the core. This gas then expands with temperature resulting in an increase of in-core pressure as the cure cycle progresses $[6,8]$. In this study, however, pressure build up in the core was not a reality for most experiments. For the constant temperature experiments, those in Section 5.1 and Section 5.3, vacuum was continually applied during the heating phase. Therefore any buildup of pressure was constantly being vented while the prepreg remained porous. Eventually the set temperature was achieved and autoclave pressurization began. At a compaction pressure of $1 \mathrm{~atm}$, the bag was vented to atmosphere, resulting in a rise of in-core pressure to a similar level. Since temperature was held constant for the remainder of the cycle, the in-core pressure would not have increased further. In the case of the constant pressure experiments (Section 5.2), heating was applied after venting of the vacuum bag. However, core movement occurred only minutes later, therefore in-core pressure was not allowed to significantly increase. This matches results presented by Brayden and Darrow [8], where it was shown that core movement will preferentially occur early in the cure cycle before in-core pressure is allowed to sufficiently build up. In fact, a slight increase in in-core pressure is observed between onset and collapse for the $325 \mathrm{kPa}$ experiment. This may be due to an increase in temperature between the two points or a reduction in cell volume due to crush. The same result is not seen for the $600 \mathrm{kPa}$ sample. The change in temperature 
between onset and collapse is much less for this sample however. 


\section{Chapter 6}

\section{Summary and Improved Model}

This body of work aims to investigate a variety of conditions involved in the processing of sandwich panels that may influence core movement. The benefit to this research over previous studies is the ability to capture core movement as it happens in-situ. Video footage provides a direct look into how core movement initiates and then progresses through the panel. Considering the results and observations outlined in the previous section, an improved mechanical model is suggested.

\subsection{Drawback of current models}

Previous work mentioned in Section 2.3.1 list three major factors that are involved in core movement. They are friction, core and ply stiffness, and the geometry of the core. Core stiffness is a combination of the material stiffness as well as in-core pressure. Friction is broken down into friction between prepreg plies $\left(F_{p-p}\right)$, friction between prepeg and the tool $\left(F_{p-t}\right)$, and friction between prepreg and the bag $\left(F_{p-b}\right)$. Geometry constitutes the chamfer angle and the height of the core. As presented in this study, there are a number of considerations that should be considered in the core movement phenomena. They are outlined below.

\section{Temperature effects on friction}

In previous studies [28, 35, 42], the prepreg-bag friction term has always been negated. However, in Section 5.1, it is shown that the processing temperature affects the frictional interfaces. At high temperature, the slip interfaces shift from between prepreg plies to between the immediate bag and toolside surfaces. Therfore prepreg-bag friction becomes of concern. That said, as noted in Section 5.2, if pressure is increased immediately, core movement is likely to occur before high temperature conditions are achieved. Therefore, temperature-induced changes in the frictional resistance of core movement only become of concern if panels are brought to high temperature prior to pressure application. For typical cure cycles where high pressure is introduced early on, temperature affects on core movement can be negated. 


\section{Ply buckling}

An important factor to consider is wrinkle formation through ply buckling. As crush progresses, plies within, and ahead of, the crush front buckle out of plane along the bag and toolside surfaces. In other words, the compressive strength of the plies offer additional resistance to crush which has largely been glanced over. Previously this has been combined with the material resistance of the core and in-core pressure under the overarching term $F_{\text {stiffness }}[28,42]$. However, since the bending modulus of plies evolves with cure to become a significant value, it is separated here. Assuming the length of ply across each honeycomb cell acts as an unsupported Euler beam, then the resistance to crush offered by the plies can be approximated according to the Euler buckling criterion shown below.

$$
P_{c r i t}=\frac{\pi^{2} E_{p l y} I}{\left(K c^{2}\right)}
$$

Where $c$ is the unsupported length of ply, equivalent to the honeycomb cell size, and $\mathrm{K}$ is the column effective length factor determined by the end condition of the plies at the cell walls. $E_{p l y}$ of an uncured ply is quite low. However, as cure progresses this factor becomes more significant. Therefore, the resistance offered by ply buckling increases with the DOC. In fact, the reason the $180^{\circ} \mathrm{C}$ experiment does not continue crushing is because ply stiffness increases significantly.

\section{Use of a surfacing film}

Unlike in previous models, slippage across the prepreg-tool interface is less likely than slippage between prepreg layers for the layup used. This is evidenced by the fact that ply 1 (the first ply on the toolside) remains stationary in all but two tests (i.e. the $600 \mathrm{kPa}$ and $180^{\circ}$ tests). Furthermore, in the $600 \mathrm{kPa}$ test, ply 1 moves considerably less than the other plies. This suggests that surfacing film increases the frictional resistance across the toolside interface. Recall also that vacuum was applied following placement of ply 1 during layup. This too, may have acted to increase the frictional resistance across the ply 1 -surfacing film interface. In any case, the prepreg-tool friction term, $F_{p-t}$, can be ignored in determining the onset of core movement. Just as in the Hsiao model, slippage across the bagside surface seems to preferentially occur between prepreg layers rather than across the prepreg-bag interface. Therefore, the prepreg-bag friction term, $F_{p-b}$ can also be disregarded. These statements are no longer true at high processing temperatures, however.

Technically, an additional frictional interface exists between the surfacing film and the tool, $F_{s f-t}$. However, in no case did the surfacing film move. Therefore, it can be said that for tacky surfacing films, such as the one used here, the friction between surfacing film-tool is much greater than between prepreg-surfacing film. Thus, $F_{s f-t}$ can be neglected and only the interface between prepreg-surfacing film need be considered along the tool. For simplicity, the prepreg-surfacing film interface is referred to as the prepreg-tool interface, $F_{p-t}$. For boardy surfacing films (i.e. not tacky) this relation may no longer apply and $F_{s f-t}$ should be considered. 


\section{Core-ply friction}

Friction between the core and prepreg is typically ignored, as the plies adjacent to the core are assumed to be bonded with the core. However, as seen for for the $45^{\circ}$ chamfer and CMS samples in Figure 5.17, it is possible for slip to occur across this interface. That said, slippage across the core-prepreg interface was not seen in any of the other samples. Moreover, considering that the core did not exhibit notable movement in the full tie-down case shows that interfacial slip between core and prepreg is unlikely; at least for unstabilized chamfer angles of $20^{\circ}$ and less. If friction between the core and prepreg was not significant, then despite restraining adjacent plies, the core should have collapsed in this experiment. Therefore, in considering the conditions necessary for the initiation of core movement, friction between core and prepreg $\left(F_{p-c}\right)$ is unlikely to be a factor for standard panel layups. Even in the case of CMS and steeper chamfer angles, it is unlikely that initiation of core movement occurs along the core-prepreg interface. Rather, given the extent of relative ply movement, slippage likely occurs first across a prepregprepreg interface followed later by slippage of the core itself as the crush front progresses. Video footage confirms this as the plies can be seen moving as soon as core movement initiates. That said, the core-ply interface may need to be considered for certain stabilization techniques or steeper chamfer angles.

\section{Straining of plies}

Ply slippage during core movement has traditionally been treated as a series of rigid members sliding past one another. However, the elastic modulus of uncured carbon-epoxy prepreg is relatively low. Therefore, the plies are compliant and may experience some level of strain as the core attempts to pull inward. This would result in a tension force that resists core movement. Moreover, if it assumed that all plies are strained equally prior to core movement initiation, then resistance to core movement would increase further from the core, where more plies must be considered. Hence, if the coefficient of friction is equivalent among plies, then slip will occur across the nearest ply-ply interface to the core. At this location, only one ply contributes to core movement resistance through tension. The problem can be likened to springs resting on top of one another in parallel. The resistive force due to strain energy is equal to the combined force exerted on each engaged spring. This is modeled in Figure 6.1 for the bagside. A similar scenario occurs on the toolside, but is not shown as the problem can be assumed symmetric. The core is pulled with force $F_{x}$. At each interface, there exists a friction force $F_{12}-F_{B a g}$. In addition, each ply experiences some level of strain resulting in tensile forces $F_{1}-F_{4}$. The further up slip occurs, the more plies (springs) become engaged. The force required to cause core movement is therefore a function of the magnitude of friction across the slip interface and the number of plies engaged at that interface. Considering only these two forces, the following general relation can be made in regards to core movement initiation:

$$
F_{x}>2\left[\left(F_{1}+F_{2}+\ldots+F_{n}\right)+F_{n, n+1}\right]
$$

Where, $F_{n, n+1}$ represents the magnitude of friction across the slip plane. The ' 2 ' comes from the fact that the problem is symmetric and a similar scenario develops in the toolside plies. Assuming the 


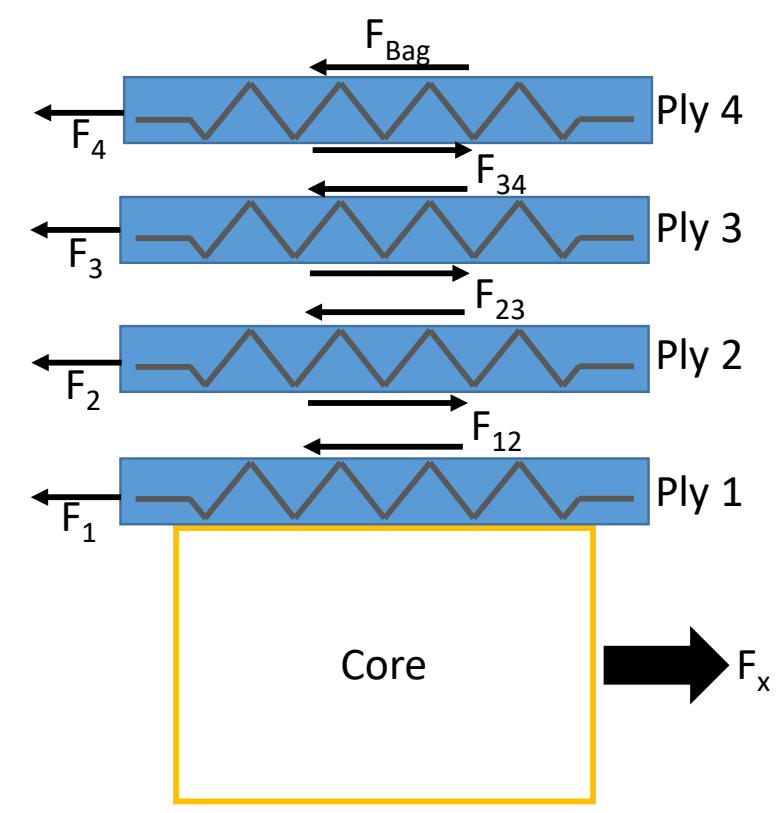

Figure 6.1: Schematic showing the tension $\left(\mathrm{F}_{1}-\mathrm{F}_{4}\right)$ developed in bagside plies as core deforms. Friction $\left(\mathrm{F}_{12}-\mathrm{F}_{\text {Bag }}\right)$ at the interfaces is provided. The plies can be approximated as springs slipping relative to one another.

tension in each ply is the same $\left(F_{T}\right)$, then the expression can be simplified to:

$$
F_{x}>2\left(n F_{T}+F_{n, n+1}\right)
$$

Where $n$ represents the number of plies above or below the slip interface.

Thus, the failure scenario requiring the least energy is slippage across the ply 1-2 interface, where only tension in ply 1 is of concern. Under this scenario, ignoring all other factors (material stiffness, incore pressure, etc.), core movement initiates when $F_{x}>2\left(F_{T}+F_{12}\right)$. Once slippage occurs, the strained plies will unload somewhat and friction will dominate.

Under high temperature conditions, slippage occurs across the bag and tool interfaces. Therefore, according to Equation 6.3, all plies are engaged and $F_{x}>8 F_{T}+F_{B a g}+F_{T o o l}$. Since the force required to cause core movement does not change much at high temperature, it implies that either the friction between prepreg-bag and prepreg-tool decreases drastically compared to prepreg-prepreg friction or the tensile contribution is minimal at high temperatures. It is likely that the latter case is the predominant factor. In the $180^{\circ} \mathrm{C}$ test, the $\mathrm{DOC}$ was quite advanced at the moment of pressure application. Therefore, plies are no longer compliant and will preferentially slip rather than experience much (if any) strain. 


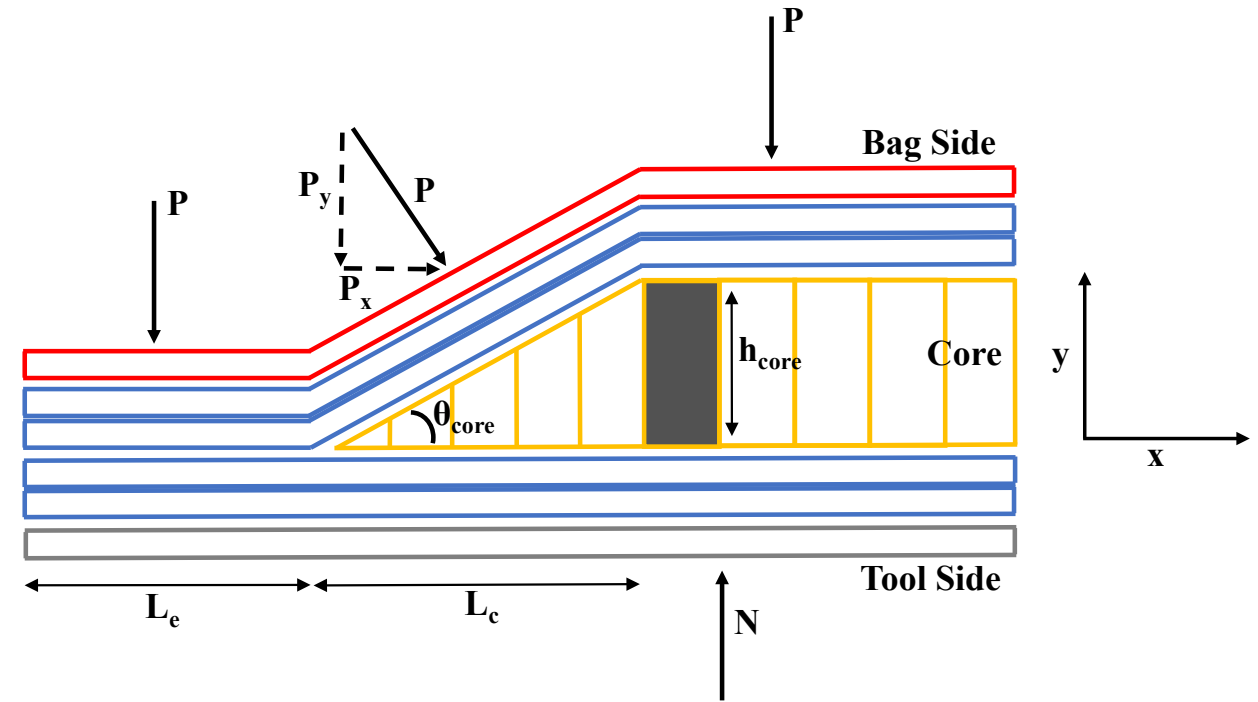

(a) Location of initial cellular collapse (shaded area).
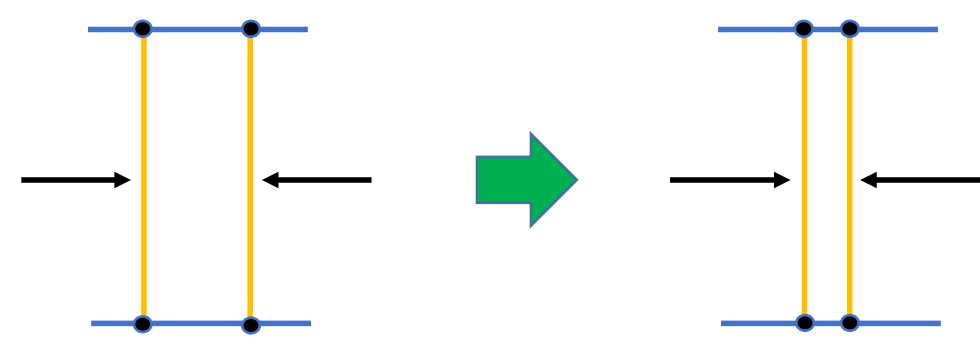

\section{Slipping}

\section{OR}
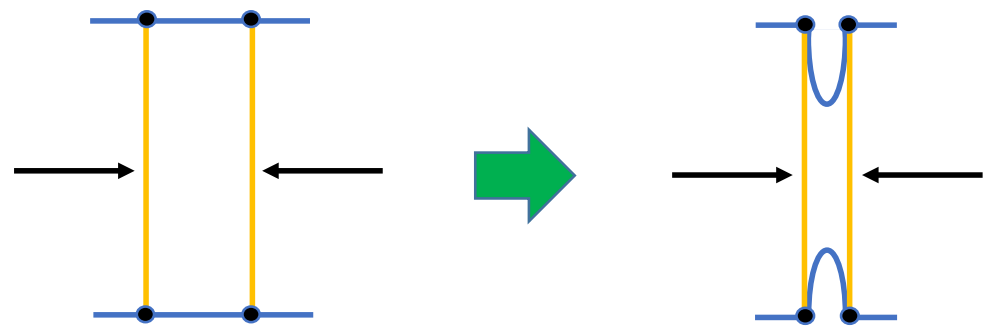

\section{Buckling}

(b) Condition for initial cellular collapse.

Figure 6.2: Schematic showing the first zone of resistance against core movement (i.e. the chamfer radius). In order for the cells to collapse, they must either slide relative to the under and overlying plies or the plies must buckle with the cells. 


\subsection{Core movement process}

\subsubsection{Conditions for initiation}

During bending, cell distortion is exhibited throughout the core. The prepreg responds in kind by deforming and creating a rough surface. Once collapse occurs the core and plies begin dragging inward. The same forces are involved in both cases, yet initiation of collapse is a localized response. To conceptualize the forces involved in core movement, it is best to look at the problem at the moment of cell collapse. It was shown that collapse of the core seems to initiate just beyond the radius. This represents the first zone of influence for resistive forces. At this location, the effective collapse area is constant and at a maximum. Looking at the problem from a $2 \mathrm{D}$ perspective, pressure is distributed per unit length rather than per unit area. Therefore, the width of the core is not important. The chamfer angle of the core only serves to increase the horizontal component of pressure. This is presented in $6.2 \mathrm{a}$ with the location of initial cell collapse shaded.

Focusing on this single cell, it can be said that in order for collapse to initiate the cell must slide relative to the adjacent ply or the ply must buckle with the cell (6.2b). In each experiment performed, buckling seems to be the preferred mode of initial collapse. Relative sliding only becomes of concern for steep chamfer angles or CMS during progression of core movement. Therefore, the forces of concern in this zone are those generated from the core, $F_{c o r e}$, and buckling resistance of the plies. The core resists crush by means of material stiffness and in-core pressure.

During buckling, plies along the laminate edge are pulled inward. This gives rise to resistance through frictional interactions and ply tension, as mentioned previously. Either the plies slip in a purely frictional manner or the plies are strained. In the later case, slippage must still occur along the adjacent surfaces to allow the plies to deform. A combination of the two modes is also possible. The laminate edge can therefore be taken as the second zone of influence for resistive forces. This is highlighted in Figure 6.3.

Therefore, the condition for core movement initiation can be summarized as follows:

$$
F_{\text {applied }}>\left(F_{\text {resist }}\right)_{\text {Initiation }}
$$

Where,

$$
F_{\text {applied }}=f\left(P_{\text {net }}, h_{\text {core }}, \theta_{\text {core }}\right)
$$

And,

$$
\left(F_{\text {resist }}\right)_{\text {Initiation }}=f\left(\text { Friction }, F_{T}, F_{\text {core }}, P_{\text {crit }}\right)
$$

If the laminate edge is taken to represent the zone of primary influence for frictional interactions, then the length $\left(L_{e}\right)$ of the laminate edge becomes important. Ignoring gravitational effects, the normal force acting on this zone $\left(N_{\text {lam }}\right)$ is equal to the net pressure multiplied by the laminate edge length. 


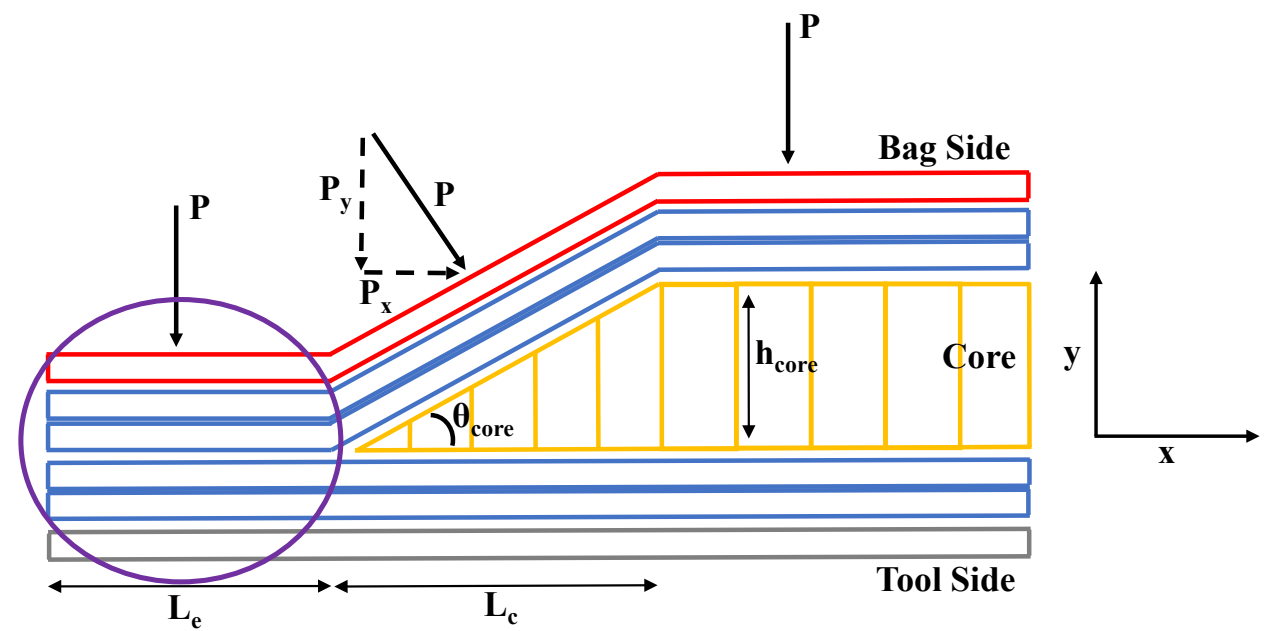

(a) Resistance develops along the laminate edge (purple circle) as plies are pulled inward.

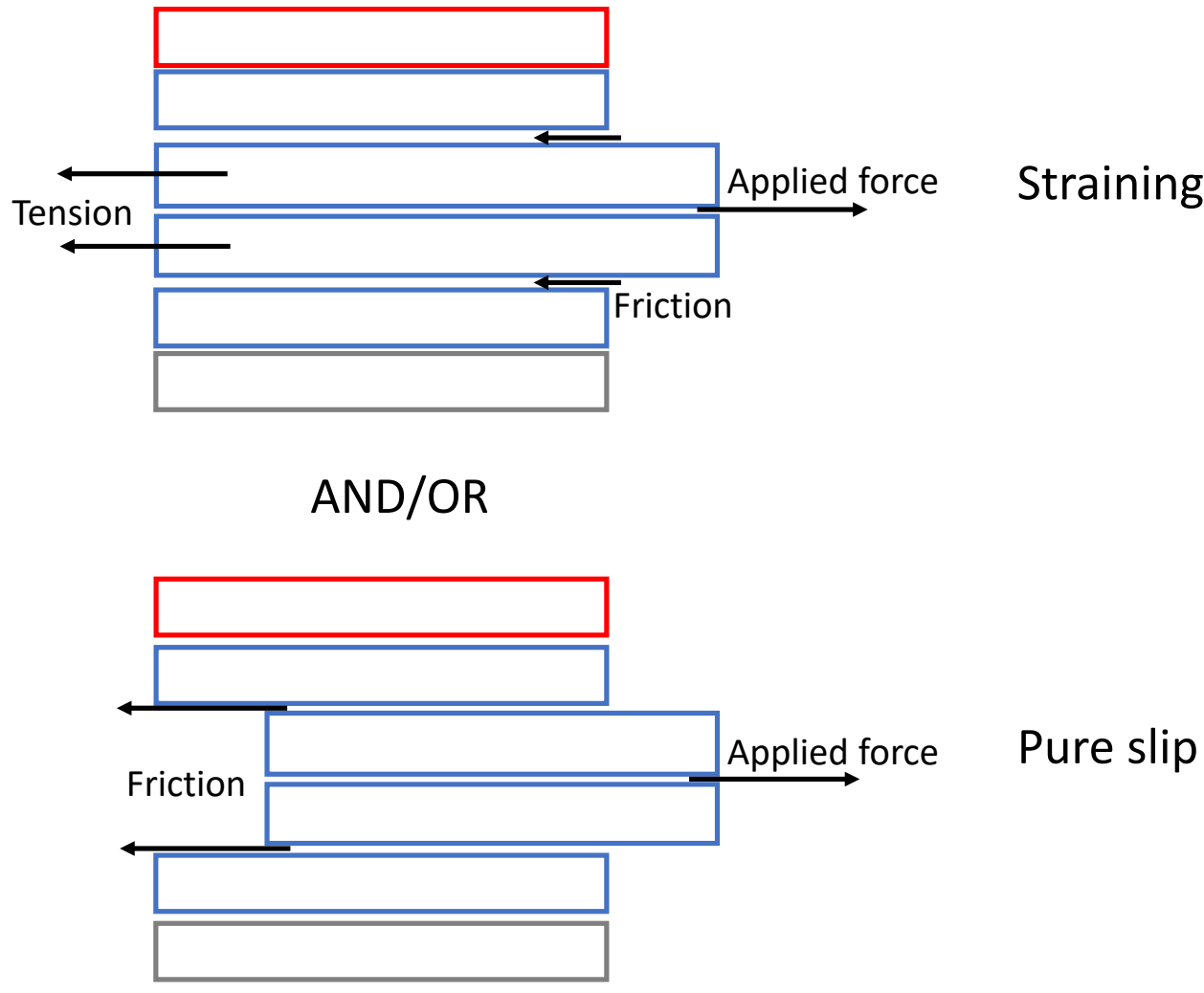

(b) Condition for ply movement.

Figure 6.3: Schematic showing the second zone of resistance against core movement (i.e. the laminate edge). In order for the plies to move, they must either stretch and/or slip across an interface. 


$$
N_{\text {lam }}=P_{\text {net }} L_{e}
$$

Coloumb fricton is proportional to the normal force according to Equation 2.16. Therefore Equation 6.7 is important in understanding the magnitude of frictional resistance between prepreg layers as well as across the prepreg-bag interface and across the prepreg-tool interface. It also highlights the importance of geometry of the panel itself. A larger laminate edge allows for an increase in frictional resistance. Each one of these friction regimes can therefore be said to be a function of the net pressure, laminate edge length, and their respective friction coefficient. In considering only the conditions necessary for the initiation of core movement, hydrodynamic shear stress can be assumed negligible. Hydrodynamic effects are proportional to the relative velocity between layers. Therefore, prior to movement, $\tau_{\text {hyd }}$ does not influence frictional resistance. That is not to say that viscous effects do not contribute to frictional resistance pre-movement, however. Rather, an adapted friction coefficient is used that evolves with cure. It is itself a function of viscosity and dry friction and must be experimentally determined for the given processing conditions. Once movement initiates, hydrodynamic effects should be considered. The following relations can, therefore, be made concerning the frictional interfaces during initiation of core movement:

$$
\begin{aligned}
& F_{p-b} \approx P_{n e t} L_{e} \mu_{p-b} \\
& F_{p-p} \approx P_{n e t} L_{e} \mu_{p-p} \\
& F_{p-t} \approx P_{n e t} L_{e} \mu_{p-t}
\end{aligned}
$$

Equations 6.8.6.10 show that while pressure is the driving force for core movement, it also contributes to core movement resistance by increasing the normal force along the laminate edge. However, increasing compaction pressure has also been shown to reduce the coefficient of friction [34, 35]. Regardless, a longer laminate edge results in an increase in friction force. An infinitely long laminate edge therefore represents a system with infinite friction. Tie-down plies can be represented in this way. That is, using tie-downs is mathematically similar to drastically increasing the laminate edge length. The equations are listed as approximate, as frictional interactions across the length of the chamfer edge, $L_{c}$, are ignored.

Core movement initiation can, therefore, be simplified in an easy-to-understand way. Since movement originates at the radius, the core can be represented as a single cell of height $h_{\text {core }}$. The plies can be thought of as a single entity sliding against two fixed interfaces, wherein the interfaces depends on the processing conditions. In order for the cell to crush it must overcome the resistive forces outlined in Equation 6.6. That is, frictional resistance, ply tension, resistance of the core, and buckling resistance across the bag and toolside surfaces. Such a model is presented in Figure 6.4. The magnitude of the friction forces are dependent on the pressure, laminate edge length, and friction coefficient according to 


\section{Zones of resistance}

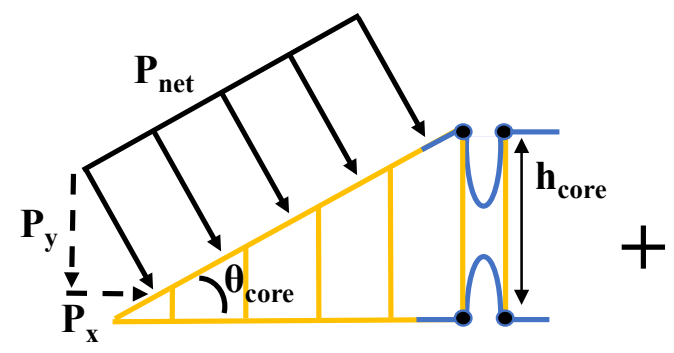

1. Chamfer radius

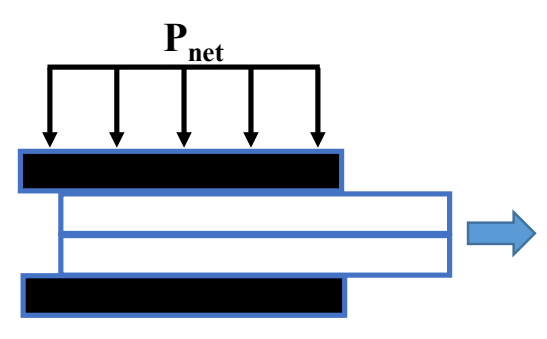

2. Laminate edge

\section{Combined resistive force response}
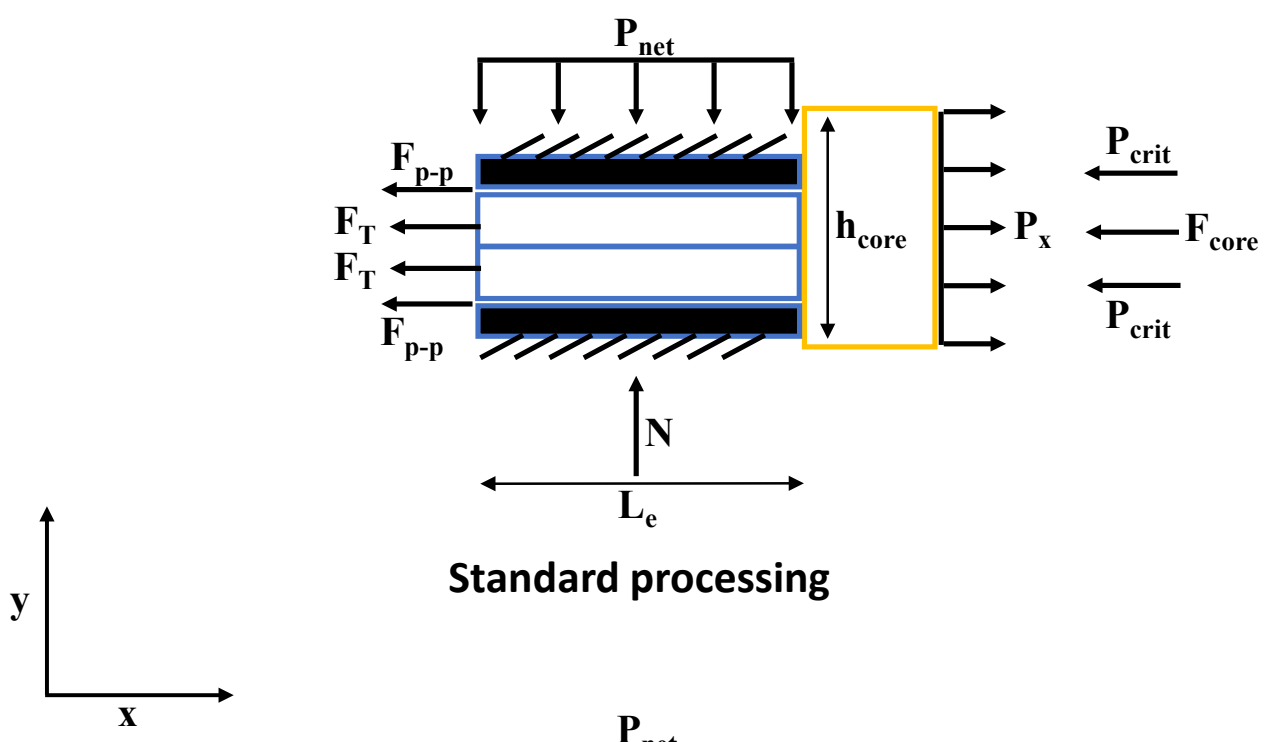

\section{Standard processing}

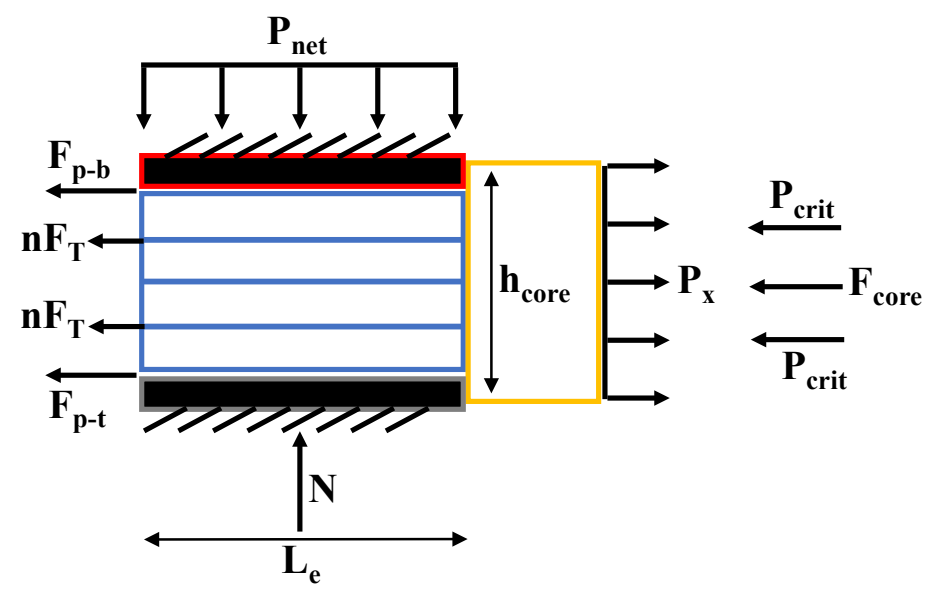

High temp processing

Figure 6.4: Core movement initiation for standard and high temperature processing. Each model incorporates the use of bagside PTFE release film and a tacky surfacing film across the tool. 
Equations 6.8 6.10. The driving force comes from the horizontal component of pressure, $P_{x}$, and can be denoted as follows:

$$
F_{x}=P_{\text {net }} \sin \theta_{\text {core }}\left(h_{\text {core }}\right)
$$

Using this model, the condition for core movement initiation becomes:

\section{Standard processing}

$$
P_{\text {net }} \sin \theta_{\text {core }}\left(h_{\text {core }}\right)>2 F_{T}+2 F_{p-p}+2 P_{\text {crit }}+F_{\text {core }}
$$

$* P_{c r i t}$ is likely negligible.

\section{High temperature processing}

$$
P_{\text {net }} \sin \theta_{\text {core }}\left(h_{\text {core }}\right)>2 n F_{T}+F_{p-b}+F_{p-t}+2 P_{\text {crit }}+F_{\text {core }}
$$

$* F_{T}$ is likely negligible.

$F_{\text {core }}$ is the combination of resistive forces due to material stiffness of the honeycomb and entrapped gas within the honeycomb. $P_{\text {crit }}$ is the force required to cause buckling of the plies, described in Equation 6.1. Figure 6.4 assume the use of bagside PTFE release film and a tacky surfacing film along the tool. If a boardy surfacing film is implemented instead, the models may need to be updated to include an additional friction term, $F_{s f-t}$, immediately adjacent to the tool. In this scenario, $F_{p-t}$ becomes $F_{p-s f}$ (i.e. friction between prepreg-surfacing film). Similarly, if the core angle surpasses $45^{\circ}$ or if stabilization techniques are used, friction between the core and prepreg may need to be considered (see Section 6.2.2).

It may be useful to define core movement as a strain requirement. Equations 6.12 and 6.13 are setup based on collapse of a single cell (i.e. core movement initiation). However, if tolerances allow for greater deformation, additional forces may need to be considered as mentioned in Section 6.2.2.

\subsubsection{Conditions for progression}

The factors concerning core movement progression are more complicated than for initiation. Since video footage does not capture individual ply movement as it happens, patterns can only be inferred post-processing. The focus of this paper is not to delve into the physics of core movement progression. As such, this section is kept brief with key points highlighted.

Following initiation, as the crush front progresses through the core, hydrodynamic effects need to be considered. This is particularly true for low temperature processing, where resin viscosity contributes significantly to core movement resistance. At high temperature, hydrodynamic lubrication does not seem to play as much of a role, and can likely be negated. 


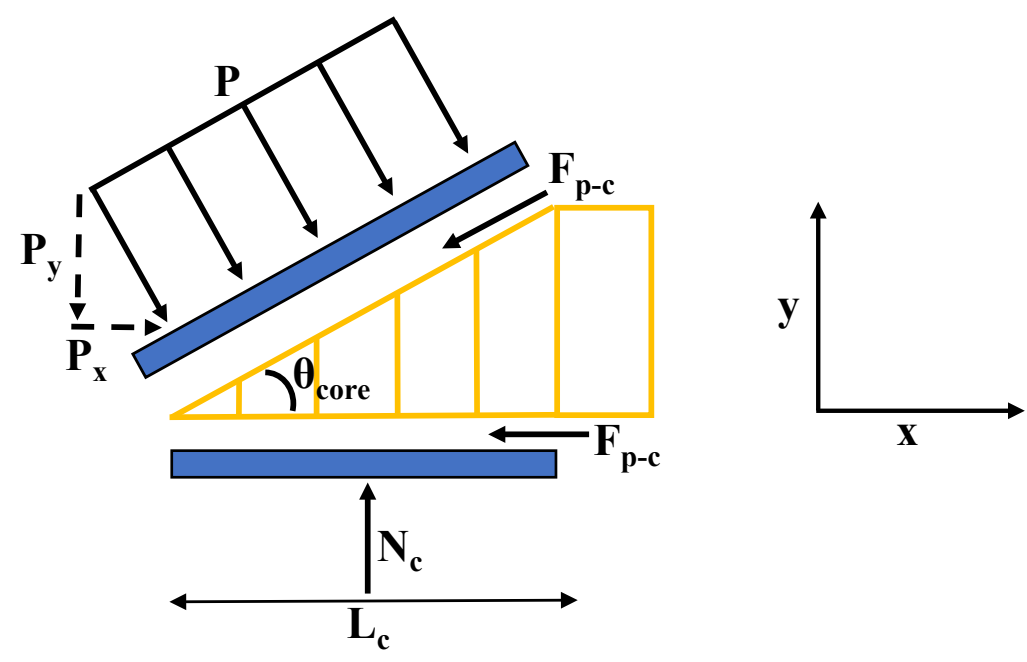

Figure 6.5: Schematic showing friction across the core-ply interface.

As described in Figure 5.31, cell collapse originates at the radius and moves inward. The crush front then shifts to along the chamfer edge before shifting back to beyond the radius. This continual shifting of collapse location can likely be attributed to densification increasing the resistance to crush. Relative density of cells then becomes an important attribute in considering core movement progression, as it effectively changes the honeycomb's stiffness.

Therefore, in general, the resistive forces are not only a function of friction, ply tension, core material stiffness/in-core pressure, and bending strength of plies, as is the case for initiation; but also hydrodynamic shear force and relative density. This is outlined in Equation 6.14. The factors controlling the applied force don't change.

$$
\left(F_{\text {resist }}\right)_{\text {Progression }}=f\left(\text { Friction }, F_{T}, F_{\text {core }}, P_{\text {crit }}, \tau_{\text {hyd }}, \frac{\rho}{\rho_{s}}\right)
$$

Ply tension likely plays less of a role in progression, as plies may unload once slippage occurs. It is still considered in Equation 6.14, however. The "friction" term includes the same three frictional interfaces as for initiation. However, for steep chamfer angles $\left(>20^{\circ}\right)$ or CMS, core-ply friction $\left(F_{p-c}\right)$ must also be considered, as the core can slide relative to the plies. In the case of CMS, this is only true along the toolside interface. Friction between the core and adjacent plies is of concern over the chamfer edge. The toolside effective length in this zone is represented as $L_{c}$ in Figure 6.2a. Bagside friction over the chamfer edge differs from toolside friction as the interface between core and prepreg is not under pure shear as is the case on the toolside. The normal force $\left(N_{c}\right)$ acts across the horizontal length of the chamfer edge for the toolside plies, and across the chamfer itself for the bagside plies. A schematic is provided in Figure 6.5.

$$
\left(N_{c}\right)_{\text {toolside }}=P_{\text {net }} \cos \left(\theta_{\text {core }}\right) L_{c}
$$




$$
\left(N_{c}\right)_{\text {bagside }}=\frac{P_{\text {net }} L_{c}}{\cos \left(\theta_{\text {core }}\right)}
$$

Therefore,

$$
\begin{gathered}
\left(F_{p-c}\right)_{\text {toolside }} \approx P_{\text {net }} \cos \left(\theta_{\text {core }}\right) L_{c} \mu_{p-c} \\
\left(F_{p-c}\right)_{\text {bagside }} \approx \frac{P_{\text {net }} L_{c} \mu_{p-c}}{\cos \left(\theta_{\text {core }}\right)}
\end{gathered}
$$

Just as with initiation, only the weakest frictional interfaces above and below the core are responsible for slippage. Therefore, for standard processing, either "friction" refers to two prepreg-prepreg interfaces, a single prepreg-prepreg interface and single core-prepreg interface, or both core-prepreg interfaces. As crush progresses down the chamfer edge, the effective length of the chamfer edge is reduced.

Finally, directionality should also be considered in dealing with core movement progression. The mode of collapse is different between the ribbon and non-ribbon directions, such that ribbon deformation typically progresses faster. As a result, the level of densification and corresponding stiffness vary along the two directions. Ribbon and non-ribbon effects can be negated in considering initiation of collapse as the effects are small compared to other terms. Moreover, in-situ data confirms that collapse occurs simultaneously in both directions.

\subsection{Processing window}

It is beneficial for manufacturers to understand the processing conditions that lead to core movement. While only one of each type of experiment was performed in this study, the results are still useful in understanding the approximate range of pressure and temperature at which core movement may occur. The model outlined in Figure 6.4 allows for speculation into the failure conditions outside of the experimental results. Figure 6.6 is an attempt to capture the processing conditions necessary for core movement for a chamfer angle of $20^{\circ}$. The curves were generated using results from Section 5.1 and Section 5.2 even though the experiments were conducted slightly differently (i.e. holding pressure vs holding temperature). Vertices represent actual data points along the curves. The bottom and top lines represent onset and collapse respectively. Between these two lines is the critical zone, wherein core movement initiates. Additional experimental results from Section 5.3 are provided at $120^{\circ} \mathrm{C}$.

Referencing the empirical failure envelope for a $20^{\circ}$ chamfer, an expected failure envelope can be generated for other chamfer angles using Equation 6.11. Figure 6.7 shows the expected failure envelope for a chamfer angle of $45^{\circ}$. The black dots represent the experimental data points at $120^{\circ} \mathrm{C}$ for the $45^{\circ}$ chamfer test. The fact that the expected curves for the $45^{\circ}$ chamfer do not fit with the experimental results at $120^{\circ} \mathrm{C}$ may infer that the resistive forces are dependent on the chamfer angle. It is possible that the strain developed in the plies is greater for the $45^{\circ}$ sample due to the increase in horizontal pressure. This would increase the tensile force component in Equation 6.12, resulting in a vertical 


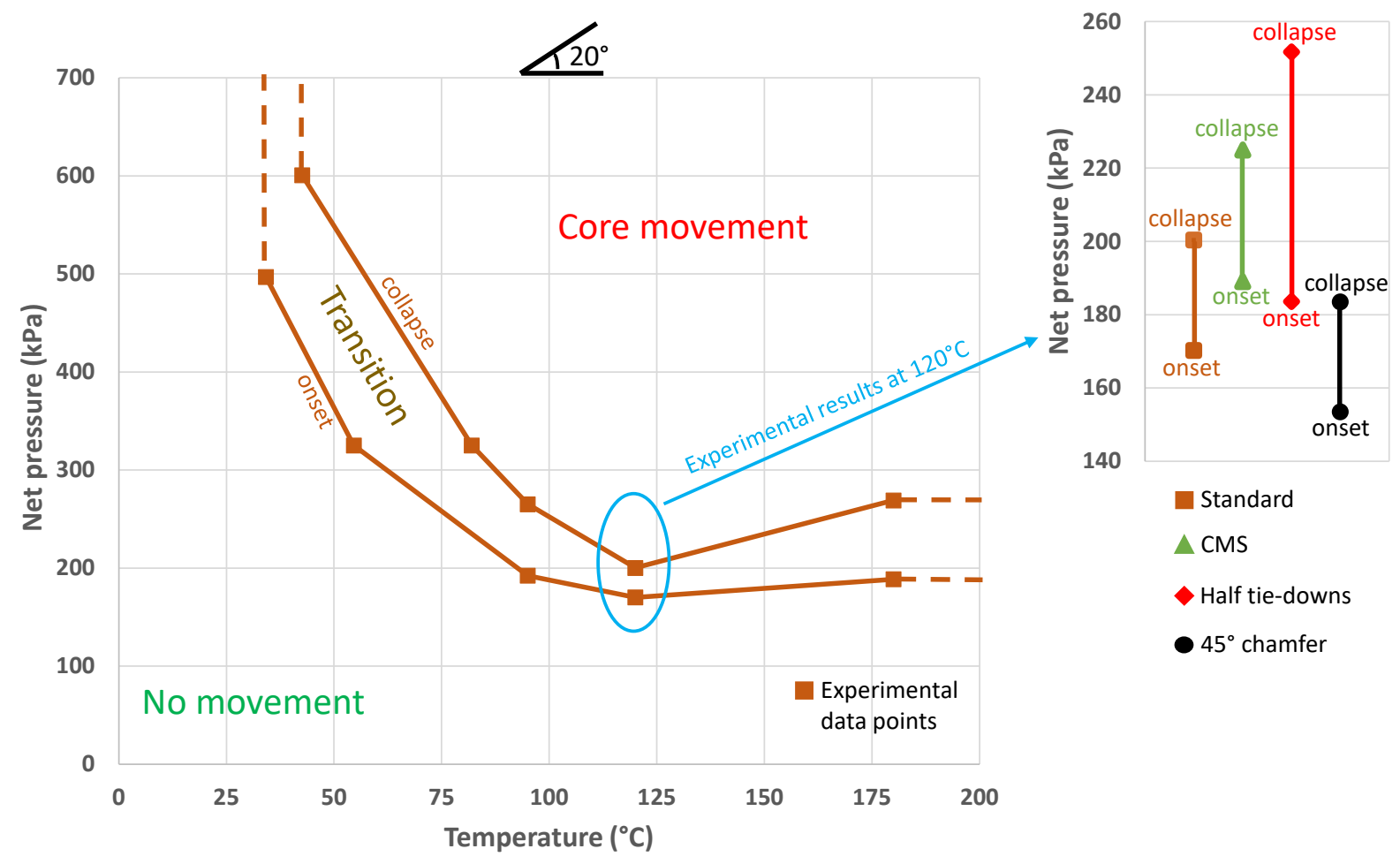

Figure 6.6: Empirical failure envelope outlining the processing conditions necessary for core movement. The curves are based on experimental results for a chamfer angle of $20^{\circ}$. Dotted lines are assumed values. Additional experimental results concerning a change in layup are provided at $120^{\circ} \mathrm{C}$.

shift in the curve of the manner seen. It is also possible that $F_{p-c}$ does actually play a role in core movement initiation for steep chamfer angles and is the source of this discrepancy (i.e. the frictional failure mode changes at steeper angles). However, more data needs to be collected in order to confirm that it isn't experimental variation. In any case, the theoretical failure envelope seems to be conservative in predicting core movement.

In order to build processing maps with predictive capabilities, it is necessary to understand how the forces involved in core movement evolve during processing. The factors relating to core movement initiation are outlined in Equations 6.12 and 6.13. Many of the resistive forces depend on temperature directly or indirectly through advancement of the DOC. Figure 6.8 outlines this dependence. The curves demonstrate the general relation that friction, core resistance, ply tension, and ply buckling have with temperature.

\section{Friction}

In these experiments prepreg-prepreg friction represents the weakest frictional interface at low temperatures. However, at higher temperatures $\left(>120^{\circ} \mathrm{C}\right)$ the interfaces of least friction shift to the prepreg-bag and prepreg-tool interfaces. This is similar to results presented by Martin et al [35], where they showed that both prepreg-prepreg friction and prepreg-tool friction decrease with temperature before leveling 


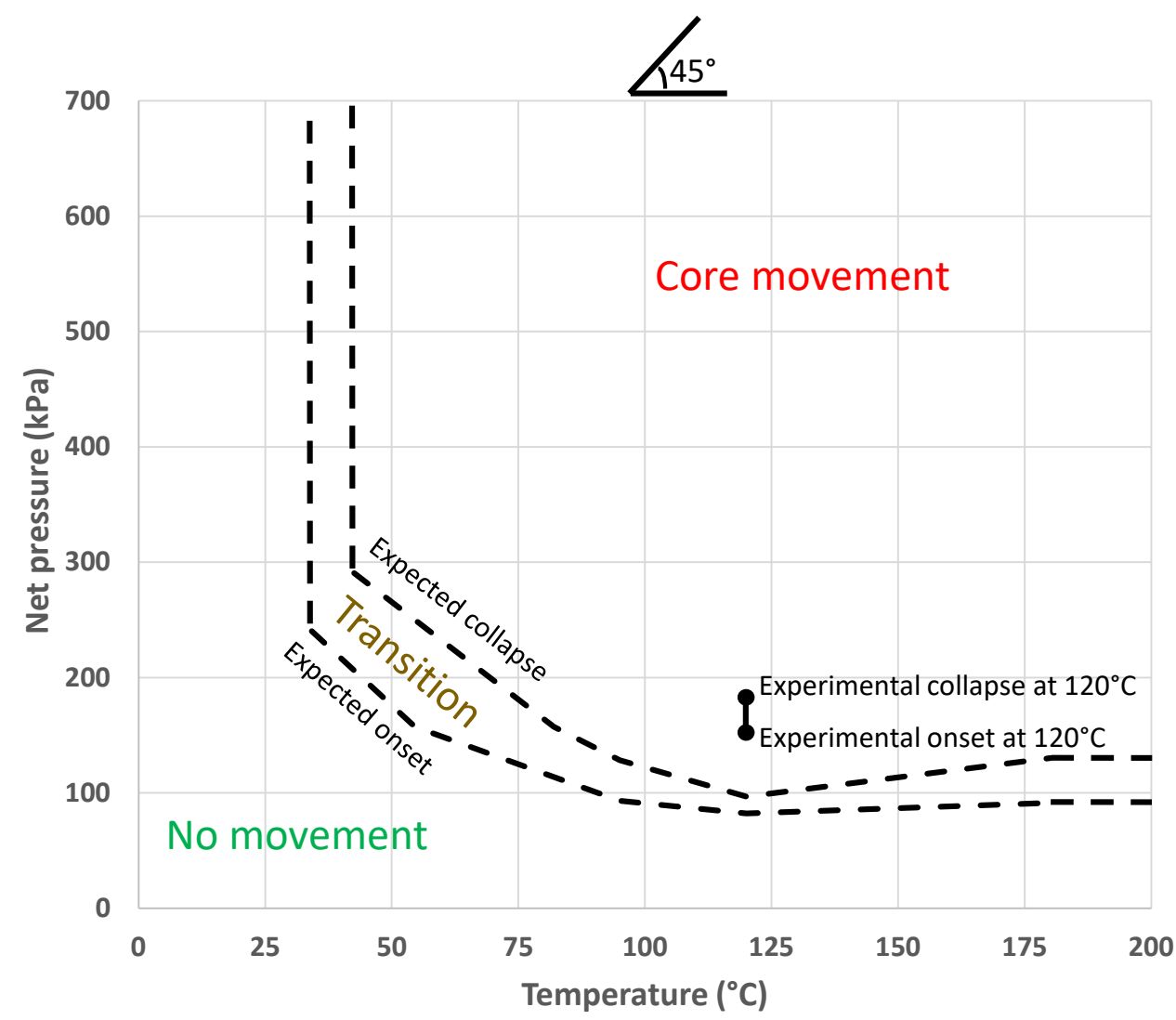

Figure 6.7: Expected failure envelope for a chamfer angle of $45^{\circ}$. The experimental results at $120^{\circ} \mathrm{C}$ are also provided.

off or slightly increasing due to fiber intermingling. However, the leveling of the curve occurs earlier for prepreg-prepreg friction than for prepreg-tool friction. As a result, at high temperature, prepreg-tool friction is considerably less than prepreg-prepreg friction. They did not measure friction between the prepreg and bag however. Combining this understanding with the results presented in this body of work gives a friction curve as presented in Figure 6.8a.

\section{Core resistance}

As mentioned in Section 5.4, in-plane mechanical properties of NOMEX ${ }^{\circledR}$ honeycomb are low. Hence, the majority of core resistance offered during core movement comes from the in-core pressure. Figure $6.8 \mathrm{~b}$ outlines the shape of the resistance curve. The material resistance of the core is denoted as $F_{\text {honeycomb. }}$. As temperature is increased, gas pressure within the core $\left(F_{\text {in-corepressure }}\right)$ increases linearly according to the ideal gas law. In reality, the slope of the curve does vary slightly due to evaporation of moisture [6, 42]. 


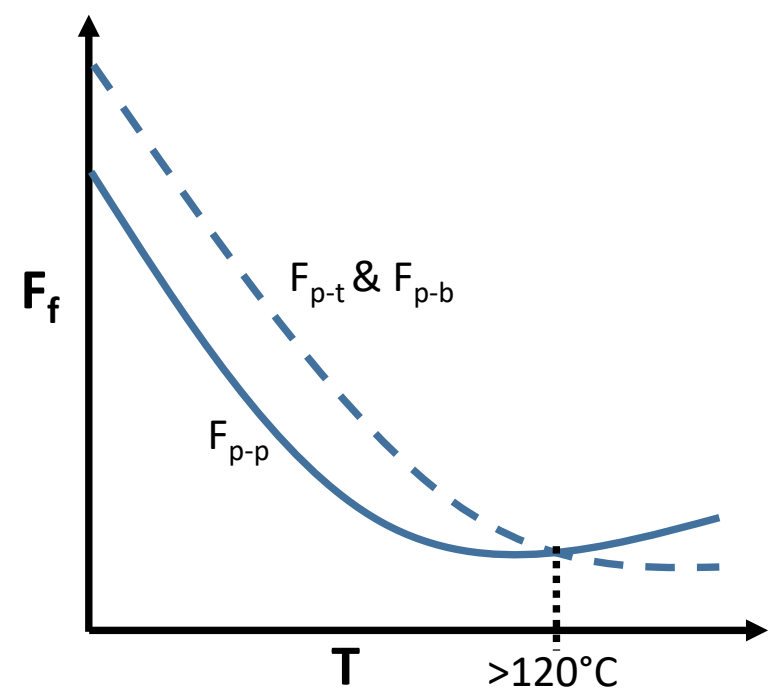

(a) Influence of temperature on frictional resistance

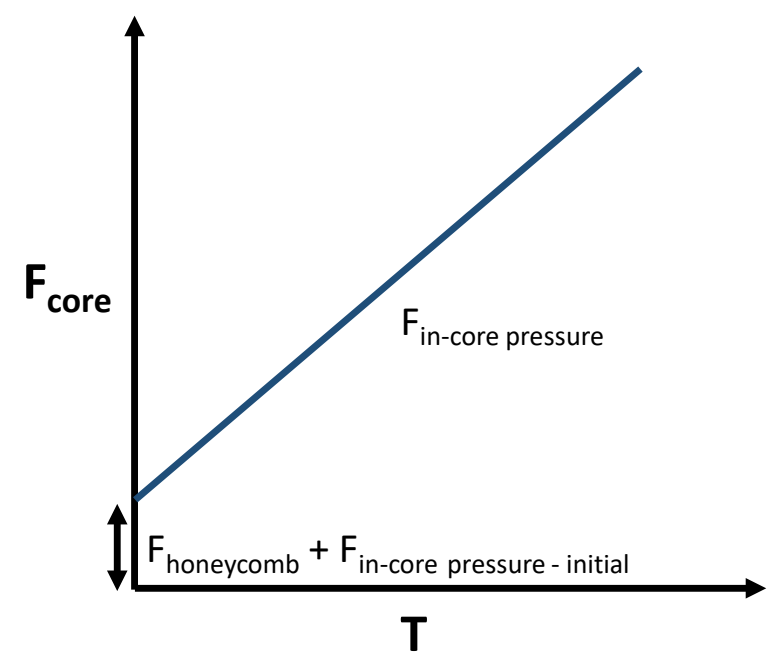

(b) Influence of temperature on core resistance
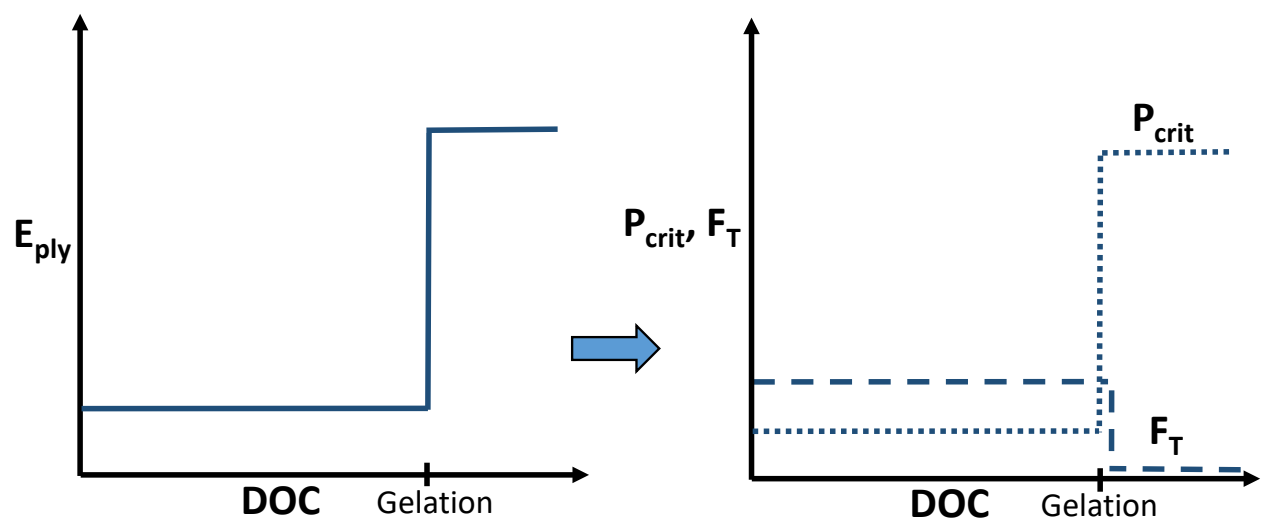

(c) Influence of DOC on tensile and buckling resistance offered by plies

Figure 6.8: (a-b) General evolution of resistive forces with temperature and (c) DOC 


\section{Ply tension and buckling}

Temperature does not affect the modulus of the ply itself, but rather through advancing the DOC. It is generally accepted that the bending and tensile modulus of an uncured ply is low. Upon gelation, the moduli increase rapidly to approach that of the tensile modulus of the fibers. This can be shown using simulation software [3]. This has opposing effects for resistance due to strain buildup and resistance due to ply buckling. Initially, when the modulus is low, the plies experience strain which contributes to core movement resistance by means of a tensile force. However, this force is proportional to the modulus of the plies. Therefore, while tensile force contributes to core movement resistance, its contribution per ply remains relatively low. As the $\mathrm{DOC}$ advances, plies become less compliant and will preferentially slip rather than experience strain during core movement. However, with stiffer fibers, the resistance offered by ply buckling increases drastically. This is reflected in Figure $6.8 \mathrm{c}$.

In the experiments performed, failure of the panels typically occurs before gelation. As such, the resistance offered by ply buckling is minimal and can likely be negated in most cases. However, in the $180^{\circ} \mathrm{C}$ test the $\mathrm{DOC}$ is around $0.5-0.6$, indicating gelation has already occured. This shows that it is possible for core movement to occur after gelation and that in high temperature cases, ply buckling cannot be negated; however, ply tension likely can. In-core pressure also becomes more significant with temperature. That said, it should not be negated at low temperatures. The initial internal core pressure may still be relatively high depending on the level of vacuum pressure applied during layup and processing. Regardless, at low temperatures friction dominates the resistive response. As temperature rises, friction decreases but remains a considerable factor. The value of ply tension is constant for low DOCs and likely lower in magnitude than that of the friction response. In fact, the failure envelope outlined in Figure 6.6 follows a similar profile to the friction model shown in Figure $6.8 \mathrm{a}$, implying core movement is largely dictated by the response of the frictional interfaces regardless of the processing conditions. However, as explained in Section 5.5.3 the manner of vacuum application prevented buildup of in-core pressure for constant temperature tests. Therefore, the heightened in-core pressure at higher temperatures did not manifest itself. In general, under typical processing cycles where pressure is set and temperature is increased, core movement occurs early enough in the cure cycle that in-core pressure does not become significant. This was shown here in the 325 and $600 \mathrm{kPa}$ tests and has been previously reported in literature [6, 8]. The significance of this is that, unless in-core pressure is specifically manipulated or the $\mathrm{DOC}$ is allowed to advance significantly before pressure application, friction will control core movement resistance; particularly prepreg-prepreg friction if a tacky surfacing film is implemented. The resistance offered by in-core pressure will, more or less, be equal to the vacuum pressure. Since ply tension does not evolve with temperature, it will only serve to shift the processing window up or down. The shape of the failure curve will still be dictated by the friction response, however.

Assuming this statement holds true, then by knowing the level of strain in the plies, the coefficient of friction can be approximated at each point on Figure 6.6. Of particular interest is the coefficient of friction at $120^{\circ} \mathrm{C}$, where the failure pressure is at an apparent minimum. An approximate upper bound is determined below by setting the strain in the plies to be zero (i.e. $F_{T}=0$ ). The values from Table 5.7 
are used for in-core pressure.

$$
P_{\text {net }} \sin \theta_{\text {core }}\left(h_{\text {core }}\right)>2{F_{T}^{*}}^{0}+2 F_{p-p}+2 \vec{P}_{\text {crit }}^{\sigma^{0}}+F_{\text {core }} \approx 2 F_{p-p}+F_{\text {in-core pressure }}
$$

Ignoring ply stiffness, material stiffness of the honeycomb, and substituting Equation 6.9 for $F_{p-p}$ yields,

$$
\left(P_{\text {net }}-P_{\text {in-core }}\right) \sin \theta_{\text {core }}\left(h_{\text {core }}\right)>2\left(P L_{e} \mu_{p-p}\right)
$$

Subbing in the appropriate values at onset and collapse gives,

$$
\left(\mu_{p-p}\right) 120^{\circ} \mathrm{C} \text { onset }=0.028
$$

And,

$$
\left(\mu_{p-p}\right)_{120^{\circ} \mathrm{C} \text { collapse }}=0.037
$$

Taking the coefficient of friction at $120^{\circ} \mathrm{C}$ as the average of these two values yields,

$$
\left(\mu_{p-p}\right)_{120^{\circ} \mathrm{C}} \approx 0.032
$$

This represents the upper bound of prepreg-prepreg friction at $120^{\circ} \mathrm{C}$ if ply tension is assumed negligible. If initiation of core movement is taken as collapse of the first cell then this seems a fair approximation, as the maximum deformation experienced by the plies is small $(\approx 3.2 \mathrm{~mm})$ relative to their length. However, this wrongly assumes no strain builds up in the plies during the bending phase of honeycomb compression.

The above friction value is equivalent to the minimum prepreg-prepreg friction value reported by Martin et al [35] for another material system (Hexcel F-593) qualified to BMS8-256. In their study, the coefficient of friction was measured under a compaction pressure of $394 \mathrm{kPa}$ with a minimum value occurring around $71^{\circ} \mathrm{C}$, versus $170-200 \mathrm{kPa}$ compaction pressure at $120^{\circ} \mathrm{C}$ in this work.

\subsection{Design improvements}

The implication of friction dominating the resistive response under standard processing is that relatively simple design changes can be made to mitigate the risk of core movement. Table 6.1 labels the factors involved in core movement that influence the applied force as well as the resistive forces. The three terms, $\theta_{\text {core }}, h_{\text {core }}$, and $L_{e}$ are geometrical in nature, which would mean the processing cycle need not be altered. Of these terms, $h_{\text {core }}$ and $\theta_{\text {core }}$ may be restricted by dimensional tolerances. However, the laminate edge can be trimmed after processing to meet design specifications. It was previously shown that prepreg-prepreg, prepreg-bag, and prepreg-tool friction are proportional to the laminate edge length. Therefore, increasing the length of the laminate edge seems a viable option for improving core movement resistance without altering the processing cycle. Larger tools would need to be implemented 
Table 6.1: Main parameters involved in the initiation of core movement.

\begin{tabular}{|c|c|}
\hline $\mathbf{F}_{\text {applied }}$ & $\mathbf{F}_{\text {resist }}$ \\
\hline$P_{\text {net }}$ & $P_{\text {net }}$ \\
\hline$\theta_{\text {core }}$ & $L_{e}$ \\
\hline$h_{\text {core }}$ & $\mu$ \\
\hline & $P_{\text {in-core }}$ \\
\hline & $E_{\text {honeycomb }}$ \\
\hline & $E_{\text {ply }}$ \\
\hline
\end{tabular}

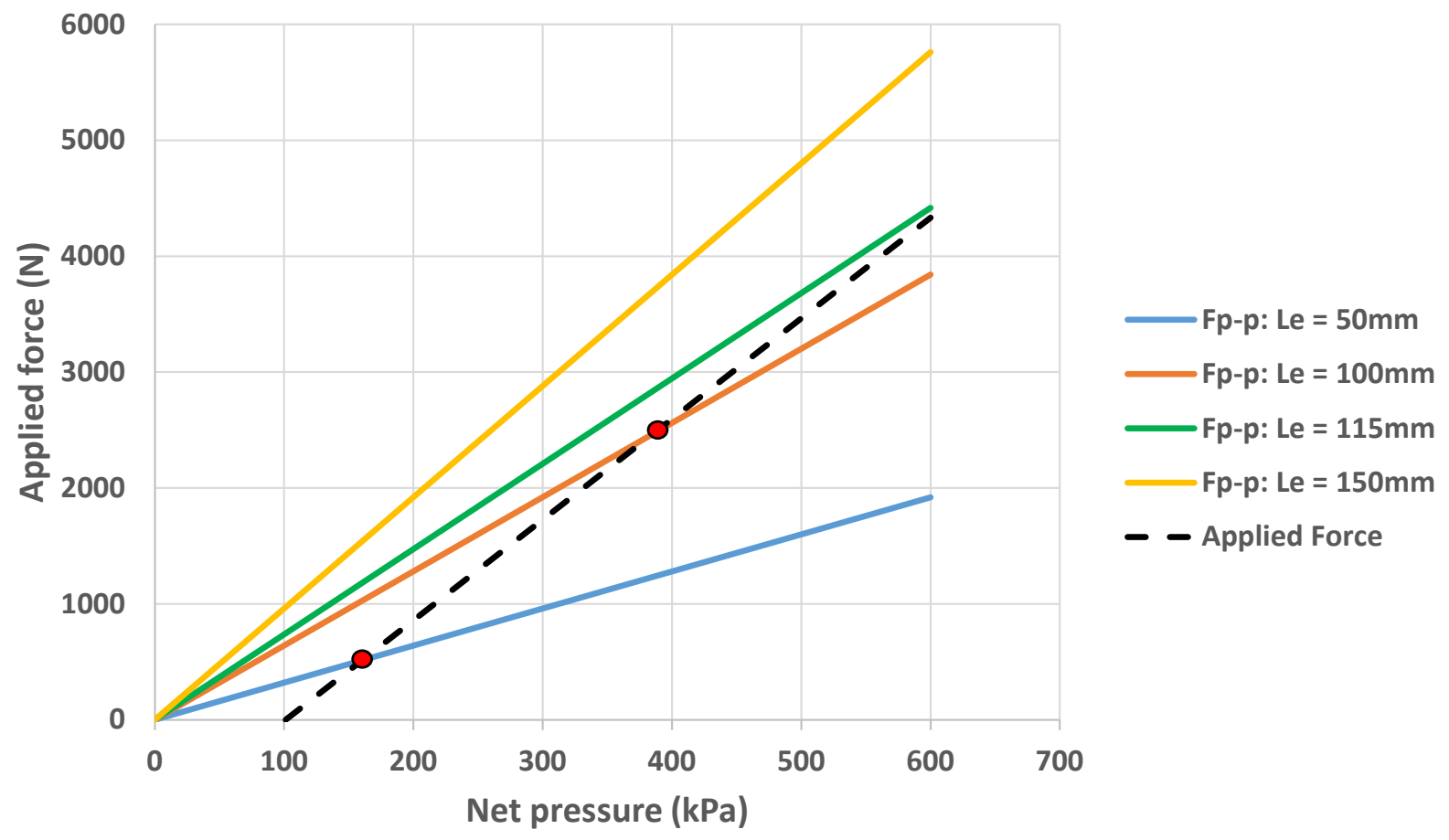

Figure 6.9: Effect of altering the laminate edge length on prepreg-prepreg friction at $120^{\circ} \mathrm{C}$. Red dots represent core movement initiation. In-core pressure is assumed to be equal to $1 \mathrm{~atm}$.

to account for the size increase; however, the idea is that grit strips and tie-downs no longer become necessary.

Figure 6.9 shows the effect increasing the laminate edge has on prepreg-prepreg friction at $120^{\circ} \mathrm{C}$. The curves were generated assuming a coefficient of friction equal to 0.032 and an in-core pressure value of $1 \mathrm{~atm}$. Buckling resistance of plies, ply tension, and material resistance of the core were assumed negligible. The point at which the applied force crosses the friction line(s) represents a conservative estimate of core movement initiation. In order to prevent core movement from occurring up to net pressures of $600 \mathrm{kPa}$, the laminate edge length would need to be at least $115 \mathrm{~mm}$; more than double the laminate edge length used in this study.

Previous attempts [6] have been made to understand the scale-up effect of a longer laminate edge on sandwich panel quality. However, the focus of these studies was in determining the viability of in-core pressurization techniques for lab-scale and large-scale parts. An understanding of how an increase in 
the laminate edge length affects core movement resistance on its own has not yet been reported.

Unlike increasing the laminate edge length, increasing the panel height and chamfer angle would act to promote core movement. The latter effect was shown in the $45^{\circ}$ chamfer test, albeit not to the extent predicted. Figures $6.10 \mathrm{a} \cdot 6.10 \mathrm{~b}$ show the expected effect of increasing these two parameters on the applied force. This is contrasted with the effect of increasing the laminate edge length. Again, the curves were generated assuming a coefficient of friction equal to 0.032 and an in-core pressure value of $1 \mathrm{~atm}$. The point(s) where the applied force lines (dotted) cross the friction lines (solid) represent the initiation of core movement for the given geometry. Maps such as these are useful in making design decisions as they allow manufacturers to construct panels to meet the processing conditions.

Increasing the core height is less severe than increasing the chamfer angle in terms of the applied force generated. That said, as the chamfer angle approaches $90^{\circ}$ the change in applied force peters out. In both cases, a laminate edge length of $150 \mathrm{~mm}$ is insufficient to prevent core movement in the most extreme cases. If both an increase in height and an increase in chamfer angle is implemented, the promotion of core movement would be even more significant. Similarly, decreasing both the core height and chamfer angle would significantly demote core movement initiation. Curves showing the effect of changing both parameters together are not provided.

\subsubsection{Recommendations}

In order to build accurate predictive maps such as those provided in Figures 6.9 and 6.10 , it is necessary to understand the exact coefficient of friction under different processing conditions. Frictional characterization tests need to be carried out at a variety of temperatures to develop a friction model for the given material system. This includes prepreg-prepreg friction, prepreg-bag friction, and prepreg-tool friction. From this, more sandwich panel tests at specific temperatures should be conducted wherein the panel is brought to failure and the failure pressure measured. Strain gauges (or alternate methods) should be implemented to measure the strain developed in the plies at initiation of core movement. This will determine the contribution ply tension plays in regards to core movement resistance. If it is insignificant in comparison to friction then it can be disregarded. At the moment, the extent of strain in the plies prior to movement is relatively unknown. The process map provided in Figure 6.6 is a good starting point, but only represents a few tests, some of which implemented a different cure cycle. If the process map can be shown to correlate well with the friction model, then it can be said that friction dominates the core movement response unless in-core pressure (or other properties) are specifically manipulated. In other words, the mechanical model would boil down to applied pressure, vacuum pressure, panel geometry, and frictional resistance. If this is the case, then accurate predictive failure maps can easily be made for a wide range of processing temperatures and pressures under standard cure cycles. This will prove a useful tool for manufactures in allowing them to design for the processing cycle, rather than make empirical based choices.

With a baseline friction model and understanding of the primary forces involved in core movement, further experiments should be conducted for specialized panels to understand their exact effect on the mechanical response at different temperatures. That is, implementation of CMS, tie-downs, steeper 


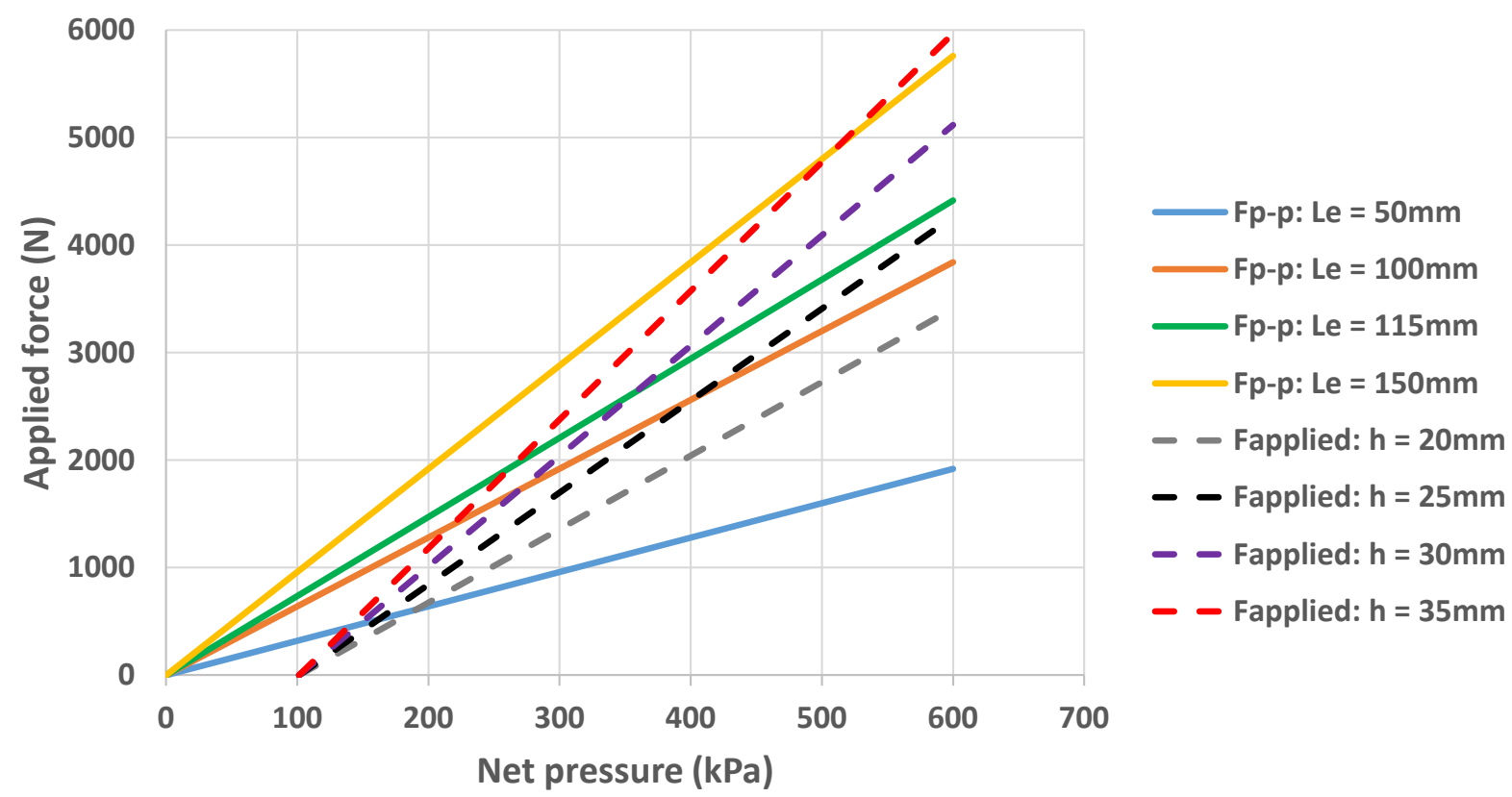

(a) Increasing $h_{\text {core }}$

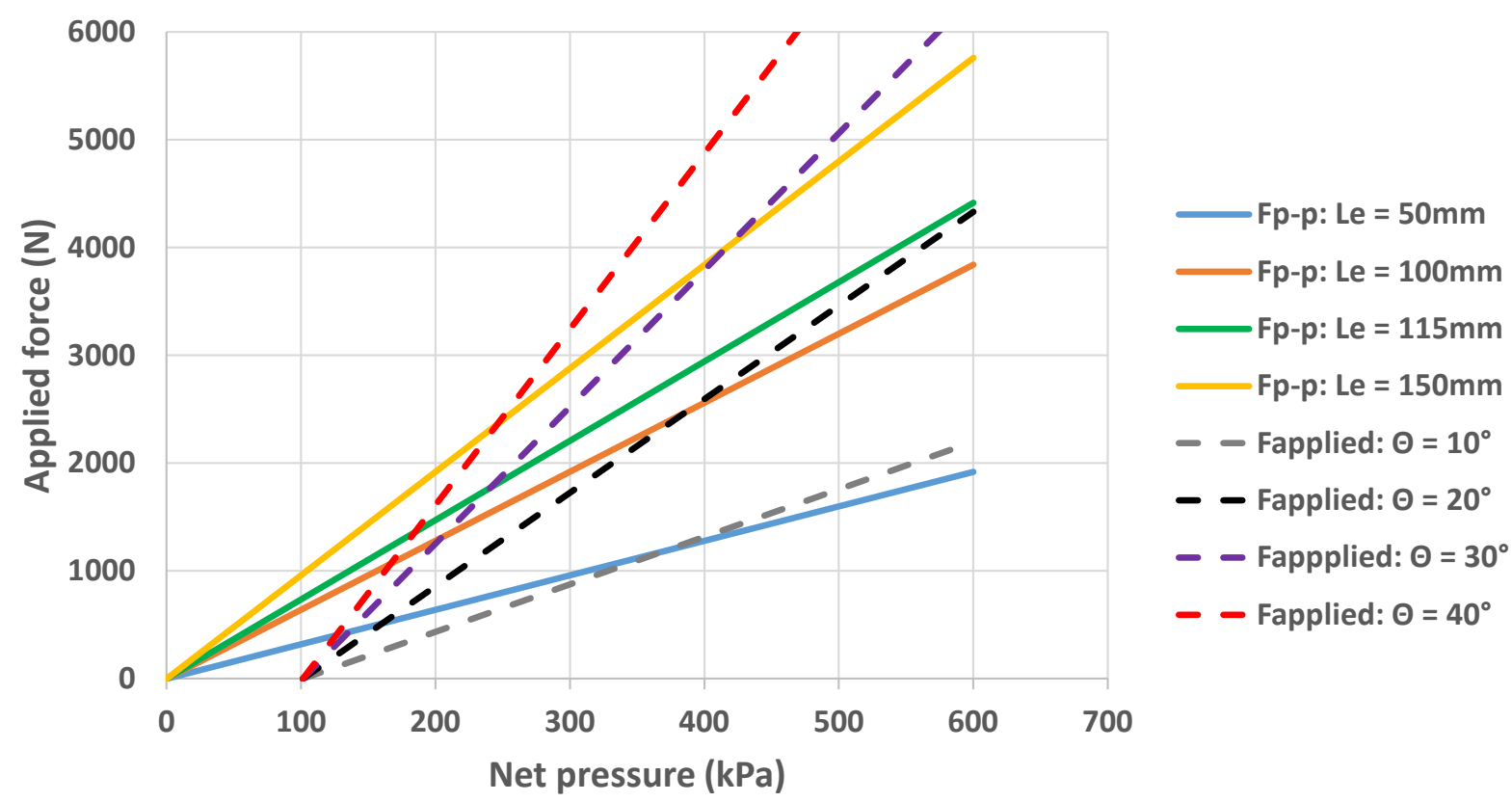

(b) Increasing $\theta_{\text {core }}$

Figure 6.10: Effect of increasing the (a) core height and (b) chamfer angle for various laminate edge lengths at $120^{\circ} \mathrm{C}$. In-core pressure is assumed to be $1 \mathrm{~atm}$. 
chamfer angles, combinations of these, etc. The results shown in this study provide a first look at how the mechanics are altered at $120^{\circ} \mathrm{C}$. However, it is relatively unknown whether the same relations would hold true at other temperatures. Moreover, it would interesting to see the results for panels with different surfacing films and film adhesives. It is suspected that changing from a tacky surfacing film to a boardy one will alter the slip interfaces. Other studies of interest would be understanding exactly when slippage occurs between core and ply for steeper chamfer angles. If it turns out that this is the first layer to slide, then altering the chamfer angle has a more significant effect than simply changing the applied force; it would also change the slip interface. The same goes for CMS.

Finally, scale-up studies investigating the effect of the laminate edge length on core movement resistance should be conducted. This may prove a relatively simple and inexpensive technique to combat core movement. 


\section{Chapter 7}

\section{Conclusions}

\subsection{Summary of results}

Core movement is a multi-faceted phenomenon that plagues sandwich panel production during the autoclave processing stage. The initiation, progression, and extent of core movement are highly dependent on the manner of panel construction and the processing conditions. This body of work investigated three main parameters influencing core movement. Namely, processing temperature, pressure, and alterations in the panel layup. Novel techniques were used to track core movement evolution in-situ, including filming the process in real time. Experimental results and observations were analyzed and a simple mechanical model was proposed.

Resistance to core movement seemed to match previous friction models, wherein at lower temperatures $\left(95^{\circ}-120^{\circ} \mathrm{C}\right)$ resistance to core movement is dependent on viscosity and decreases with temperature. At high temperature $\left(180^{\circ} \mathrm{C}\right)$, resistance to core movement changes irrespective of viscosity implying a shift from a hydrodynamic friction regime to one exhibiting boundary lubrication. In the latter scenario, the slip interfaces shift from between prepreg plies to between the tool and bag interfaces. This results in bulk movement of plies as opposed to relative movement as exhibited in the lower temperature samples. However, unless the set temperature is first achieved prior to increasing pressure, core movement will occur before high temperature conditions are reached. In fact, at high pressure (500 kPa) core movement will occur if temperature rises to just $34^{\circ} \mathrm{C}$. That said, if temperature is kept below $30^{\circ} \mathrm{C}$, core movement is unlikely to occur even for pressures around $600 \mathrm{kPa}$. This demonstrates the need for strict tolerances during processing. Similarly, pressure fluctuations of just $30 \mathrm{kPa}$ can result in drastic core movement if the processing conditions approach those necessary for core movement initiation.

Tie-downs proved effective at mitigating core movement. Single ply tie-downs were enough to eliminate the vast majority of core movement for a core angle of $20^{\circ}$, with only minor bending seen. It is suspected that utilizing double ply tie-downs will prevent all deformation. Interestingly, restraining only two edges increased the resistance to movement along the unrestrained edges. This implies that deformation along the ribbon and non-ribbon direction are somewhat coupled. Implementing Core Machining Stabilization (CMS) slightly increased the panel resistance; however, it also allowed for slippage 
across the toolside core-ply interface in the non-ribbon direction. It is suspected that further stabilization may result in increased slippage and possibly even decrease the panel's resistance to core movement. That said, collapse of the cellular structure was prevented along the stabilized zone. Finally, a steeper chamfer angle $\left(45^{\circ}\right)$ increased the applied force, resulting in earlier onset of core movement and considerable relative movement of plies. Notable slip between core and prepreg was also observed on both bagside and toolside interfaces.

Core movement initiates near the radius of the chamfer edge where the core height is maximum. From there collapse progresses inward. As densification increases beyond the radius, crush extends down the chamfer edge. Similarly as densification increases along the chamfer, cells further in the core begin collapsing at a faster rate again. This results in a concave-like chamfer edge following processing. Typically the original angle is persevered further down the chamfer. In the case of CMS, the angle is preserved along the entirety of the chamfer edge. For steeper angles, crush extends along the entire length of the chamfer edge.

Generally speaking, deformation in the ribbon direction proved greater than in the non-ribbon direction, with the exception of one sample. Ribbon deformation allows for quick progression of the crush front through the core by means of homogenous collapse. Non-ribbon deformation, however, exhibits row-by-row collapse resulting in high localized densification but typically slower progression through the core. Surprisingly, initiation of core movement occurs simultaneously in the two directions.

A simple mechanical model was created based on the observations and results attained. It includes the influence of temperature on frictional resistance and the addition of ply tension which was not previously included in core movement models. Moreover, the importance of geometry is highlighted. The sequence of events leading to core movement are also captured. First, cell collapse occurs at the radius, buckling the adjacent plies. In doing, so plies are dragged inward across the laminate edge. Resistive forces can be grouped into two categories, those occurring in the crush zone and those arising from interactions across the laminate edge. They are broken down as follows:

1. Crush zone (chamfer radius)

- Material strength of core

- In-core pressure

- Buckling strength of plies

2. Laminate edge

- Friction across the slip interface

- Tension in plies

Considering that the driving force is a function of applied pressure and panel geometry, that gives six major factors that contribute to core movement. Namely, net pressure across the vacuum bag, core strength (including in-core pressure), buckling strength of the plies, friction, ply tension, and geometry of the panel. At low DOCs, prepreg-prepreg friction seems to be the dominant mechanism controlling 
core movement resistance. If true, friction models can be used to predict the initiation of core movement. That said, ply tension has largely been overlooked and may prove an important parameter to consider. In order to truly predict the initiation of core movement for any DOC, the magnitude of each of these six parameters need to be known throughout the cure cycle for the given material system. In any case, altering geometrical features such as the length of the laminate edge in order to increase frictional resistance seems a promising method to combat core movement.

\subsection{Future work}

In order to affirm whether friction is the dominant mechanism in the core movement process, friction models need to be developed for a variety of material systems. Sandwich panels of the same material systems should be built and processed at a variety of temperatures. If core movement is shown to match the friction behavior then it can be said that friction dictates core movement unless other factors, such as in-core pressure, are artificially manipulated. Ply strain should also be measured in the sandwich panel tests to understand the level of resistance offered by ply tension. If significant, the processing window will shift upward from a purely friction-based response. Understanding ply strain prior to movement will allow for friction and tension effects to be decoupled. It is suspected that ply strain contributes to core movement resistance, however, less so than friction. As our understanding of pre-gelation behaviour improves, an accurate representation of the resistance offered by the plies can be realized. Currently, it is assumed that the plies themselves offer little resistance to core movement at low DOC;.

With regards to layup effects, cores of different geometries should be investigated. This body of work only dealt with cores of a single height. Furthermore only two chamfer angles were investigated, $20^{\circ}$ and $45^{\circ}$. It would be beneficial to evaluate the core movement model against panels with a wide range of geometries. That is, combinations of cores with varying heights and chamfer angles, as well as panels with differing laminate edge lengths. In addition, it would be useful to understand how effective tie-downs are for steeper chamfer angles such as $45^{\circ}$. Moreover, it would be interesting to note how the use of $45^{\circ}$ doubler plies influence core movement. Finally, differing surfacing films should be investigated. It is suspected that the use of boardy surfacing films will give drastically different results into where slippage initiates and how ply movement progresses. Similarly, adjusting the extent of CMS is suspected to yield different results in regards to core-ply slippage. On that same note, changing the film adhesive system will likely alter the frictional resistance between core and ply as well. Investigating each of these parameters will likely involve several studies with multiple experiments pertaining to each topic.

It is recommended that future experiments should be run under constant vacuum pressure to eliminate any effects associated with venting. This may include not applying any vacuum pressure at all or running the tests at full vacuum. Furthermore, it is suggested that ply 1 should not be debulked during layup, as this likely alters the frictional resistance across the ply 1-surfacing film interface. Although cumbersome and time-consuming, including filler plies in the layup is useful as they allow for easy tracking of relative movement between the core and prepreg. Finally, in order to eliminate gas pathways during core movement, high gauge pressure sensors wires should be used. In order to get more accurate results, 
multiple sensors should be placed throughout the core as gas pressure can vary from cell to cell. They should be placed on the underside of the core, to prevent intrusion of the film adhesive. Slicing the honeycomb to seat the pressure sensors should be avoided if possible. The duration of time which the panel is held under vacuum prior to processing should also be measured as this will influence the level of in-core pressure. 


\section{Bibliography}

[1] Daytronic, retrieved from: http://www.daytronic.com. 2019. $\rightarrow$ page 121

[2] Candylabs, retrieved from: http://www.candylabs.com. 2019. $\rightarrow$ page 123

[3] Convergent manufacturing technologies, retrieved from: http://www.convergent.ca. 2019. $\rightarrow$ pages 28, 107, 123

[4] L. Aktay, A. F. Johnson, and B.-H. Kröplin. Numerical modelling of honeycomb core crush behaviour. Engineering Fracture Mechanics, 75(9):2616-2630, 2008. $\rightarrow$ page 2

[5] H. G. Allen. Analysis and design of structural sandwich panels: the commonwealth and international library: structures and solid body mechanics division. Elsevier, 2013. $\rightarrow$ page 2

[6] A. Alteneder, D. Renn, J. Seferis, and R. Curran. Processing and characterization studies of honeycomb composite structures. In INTERNATIONAL SAMPE SYMPOSIUM AND EXHIBITION, pages 1034-1034. SAMPE SOCIETY FOR THE ADVANCEMENT OF MATERIAL, 1993. $\rightarrow$ pages 14, 17, 19, 69, 89, 90, 105, 107, 109

[7] M. F. Ashby and R. M. Medalist. The mechanical properties of cellular solids. Metallurgical Transactions A, 14(9):1755-1769, 1983. $\rightarrow$ page 7

[8] T. Brayden and D. Darrow. Effect of cure cycle parameters on $350^{\circ} \mathrm{F}$ cocured epoxy honeycomb core panels. In Proceedings of the Society for the Advancement of Material and Process Engineering - Thirty-fourth Technical Conference, 1989. $\rightarrow$ pages 3, 14, 15, 17, 18, 90, 107

[9] J. Brown and B. Ennis. Thermal analysis of nomex $\AA$ and kevlar $\AA$ fibers. Textile Research Journal, 47(1):62-66, 1977. $\rightarrow$ page 10

[10] F. C. Campbell Jr. Manufacturing processes for advanced composites. elsevier, 2003. $\rightarrow$ pages 5., 17,59

[11] Q. Chen and N. M. Pugno. In-plane elastic buckling of hierarchical honeycomb materials. European Journal of Mechanics-A/Solids, 34:120-129, 2012. $\rightarrow$ pages 7, 10

[12] D. H. Corbett and S. A. Smith. Tiedown ply for reducing core crush in composite honeycomb sandwich structure, Apr. 20 1999. US Patent 5,895,699. $\rightarrow$ page 17

[13] D. A. Crump, J. M. Dulieu-Barton, and J. Savage. The manufacturing procedure for aerospace secondary sandwich structure panels. Journal of Sandwich Structures \& Materials, 12(4): 421-447, 2010. $\rightarrow$ page 17

[14] Daytronic. 5M30 AC LVDT Conditioner Module product manual, . $\rightarrow$ pages Xv, 121, 122 
[15] Daytronic. LVDT AC, short stroke, unguided, [SUH Series] product datasheet, . $\rightarrow$ page 121

[16] C. Duffner, N. Zobeiry, and A. Poursartip. Examination of pre-gelation behavior in as4/8552 prepreg composites. In Proceedings of the American Society for Composites-Thirty-third Technical Conference, 2018. $\rightarrow$ page 14

[17] J. Dulieu-Barton, D. Crump, and D. Griffith. Effect of core crush on honeycomb sandwich panels. In Proceedings of the 9th International Conference on Sandwich Structures, 2010. $\rightarrow$ page 17

[18] DuPont ${ }^{\mathrm{TM}}$. Nomex ${ }^{\circledR}$ history, retrieved from: http://www.dupont.com/public_sector_er/en_gb/nomex_the_fibre/nomex_history.html. 2011. $\rightarrow$ page 2

[19] S. Erland, T. Dodwell, and R. Butler. Characterisation of inter-ply shear in uncured carbon fibre prepreg. Composites Part A: Applied Science and Manufacturing, 77:210-218, 2015. $\rightarrow$ pages 13,14

[20] N. Ersoy, K. Potter, M. R. Wisnom, and M. J. Clegg. An experimental method to study the frictional processes during composites manufacturing. Composites Part A: Applied science and manufacturing, 36(11):1536-1544, 2005. $\rightarrow$ page 13

[21] C. C. Foo, G. B. Chai, and L. K. Seah. Mechanical properties of nomex material and nomex honeycomb structure. Composite structures, 80(4):588-594, 2007. $\rightarrow$ page 64

[22] L. Gibson and M. Ashby. Cellular Solids: Structure and Properties. Pergamon Press, 1988. $\rightarrow$ pages $\mathrm{X}, 5,7,8,9,10,11,88$

[23] S. Heimbs, S. Schmeer, P. Middendorf, and M. Maier. Strain rate effects in phenolic composites and phenolic-impregnated honeycomb structures. Composites Science and Technology, 67(13): 2827-2837, 2007. $\rightarrow$ pages 9, 73, 88

[24] C. Hexcel. Hexweb honeycomb attributes and properties. 1999. $\rightarrow$ page 64

[25] HEXCEL ${ }^{\circledR}$. HexWeb ${ }^{\circledR}$ HRH-10, Aramid Fiber/Phenolic Resin Honeycomb, Product Data Sheet, 2017. $\rightarrow$ pages 5, 24, 64

[26] W. B. Hopkins and D. E. Hartz. Adhering tiedown plies in composite construction, Nov. 111997. US Patent 5,685,940. $\rightarrow$ page 17

[27] H.-M. Hsiao, S. M. Lee, R. A. Buyny, and C. J. Martin. Core-crush resistant fabric and prepreg for fiber reinforced composite sandwich structures, Dec. 16 2003. US Patent 6,663,737. $\rightarrow$ page 18

[28] H.-M. Hsiao, S. Lee, and R. Buyny. Core crush problem in the manufacturing of composite sandwich structures: Mechanisms and solutions. AIAA journal, 44(4):901-907, 2006. $\rightarrow$ pages $\mathrm{x}, 2,3,14,15,16,18,92,93$

[29] T. R. Kain Jr. Composite honeycomb sandwich panel for fixed leading edges, Jan. 30 2001. US Patent 6,180,206. $\rightarrow$ page 17

[30] N. A. Kalebek and O. Babaarslan. Effect of weight and apllied force on the friction coefficient of the spunlace nonwoven fabrics. Fibers and Polymers, 11(2):277-284, 2010. $\rightarrow$ page 13 
[31] J. Klintworth and W. Stronge. Elasto-plastic yield limits and deformation laws for transversely crushed honeycombs. International Journal of Mechanical Sciences, 30(3-4):273-292, 1988. $\rightarrow$ pages 9,11

[32] J. Kratz and P. Hubert. Processing out-of-autoclave honeycomb structures: Internal core pressure measurements. Composites Part A: Applied Science and Manufacturing, 42(8):1060-1065, 2011. $\rightarrow$ page 19

[33] J. Kratz and P. Hubert. Anisotropic air permeability in out-of-autoclave prepregs: Effect on honeycomb panel evacuation prior to cure. Composites Part A: Applied Science and Manufacturing, 49:179-191, 2013. $\rightarrow$ pages 18, 19

[34] Y. R. Larberg and M. Åkermo. On the interply friction of different generations of carbon/epoxy prepreg systems. Composites Part A: Applied Science and Manufacturing, 42(9):1067-1074, 2011. $\rightarrow$ pages $\mathrm{X}, 12,13,14,16,99$

[35] C. Martin, J. Seferis, and M. Wilhelm. Frictional resistance of thermoset prepregs and its influence on honeycomb composite processing. Composites Part A: Applied Science and Manufacturing, 27(10):943-951, 1996. $\rightarrow$ pages 13, 15, 16, 17, 18, 38, 92, 99, 104, 108

[36] C. Martin, J. Putnam, B. Hayes, J. Seferis, M. Turner, and G. Green. Effect of impregnation conditions on prepreg properties and honeycomb core crush. Polymer composites, 18(1):90-99, 1997. $\rightarrow$ page 15

[37] C. J. Martin and J. C. Seferis. Effect of prepreg resin composition on honeycomb core crush. Materials and process affordability- Keys to the future, pages 366-375, 1998. $\rightarrow$ pages 15, 17, 18

[38] S. D. Papka and S. Kyriakides. In-plane compressive response and crushing of honeycomb. Journal of the Mechanics and Physics of Solids, 42(10):1499-1532, 1994. $\rightarrow$ pages 7, 9

[39] S. D. Papka and S. Kyriakides. Experiments and full-scale numerical simulations of in-plane crushing of a honeycomb. Acta materialia, 46(8):2765-2776, 1998. $\rightarrow$ pages 7, 9

[40] T. Pelton, T. Schneider, R. Martin, and B. C. Airplane. Material factors influencing composite part producibility in relation to prepreg frictional measurement. In INTERNATIONAL SAMPE TECHNICAL CONFERENCE, volume 31, pages 463-477, 1999. $\rightarrow$ pages 15, 17, 18

[41] D. D. Pham, M. W. Tollan, R. M. Outzen, J. C. Lockleer, and C. G. Harris. Composite ply stabilizing method, July 23 2013. US Patent 8,491,743. $\rightarrow$ page 17

[42] D. Renn, T. Tulleau, J. Seferis, R. Curran, and K. Ahn. Composite honeycomb core crush in relation to internal pressure measurement. Journal of advanced materials, 27(1):31-40, 1995 . $\rightarrow$ pages $14,17,69,78,89,92,93,105$

[43] G. S. Springer. Resin flow during the cure of fiber reinforced composites. Journal of Composite Materials, 16(5):400-410, 1982. $\rightarrow$ page 14

[44] G. Stachowiak and A. W. Batchelor. Engineering tribology. Butterworth-Heinemann, 2013. $\rightarrow$ page 12

[45] J. Sun, M. Li, Y. Gu, D. Zhang, Y. Li, and Z. Zhang. Interply friction of carbon fiber/epoxy prepreg stacks under different processing conditions. Journal of Composite Materials, 48(5): 515-526, 2014. $\rightarrow$ page 12 
[46] S. S. Tavares, V. Michaud, and J.-A. Månson. Through thickness air permeability of prepregs during cure. Composites Part A: Applied Science and Manufacturing, 40(10):1587-1596, 2009. $\rightarrow$ pages 18,19

[47] S. S. Tavares, N. Caillet-Bois, V. Michaud, and J.-A. Månson. Non-autoclave processing of honeycomb sandwich structures: Skin through thickness air permeability during cure.

Composites Part A: Applied Science and Manufacturing, 41(5):646-652, 2010. $\rightarrow$ page 19

[48] S. S. Tavares, N. Caillet-Bois, V. Michaud, and J.-A. Månson. Vacuum-bag processing of sandwich structures: Role of honeycomb pressure level on skin-core adhesion and skin quality. Composites Science and Technology, 70(5):797-803, 2010. $\rightarrow$ pages 18, 19

[49] J. Zhang and M. Ashby. The out-of-plane properties of honeycombs. International journal of mechanical sciences, 34(6):475-489, 1992. $\rightarrow$ pages 5, 7

[50] N. Zobeiry and C. Duffner. Measuring the negative pressure during processing of advanced composites. Composite Structures, 203:11-17, 2018. $\rightarrow$ page 14 


\section{Appendix A}

\section{Appendix A Data Acquisition}

\section{A.1 LVDTs}

The LVDT's were AC-Operated Short-Stroke Unguided Armature models. They are part of Daytronic's SUH LVDT series. The specific models were DS1000SUH. "Unguided" refers to the fact that the core rod was not spring loaded within the solenoid housing. Rather, it relied on gravity for displacement. LVDT included a two meter cable containing four separate wire types. Namely, a wire pair for excitation voltage, a ground wire pair, a signal wire pair, and a sensor wire pair. The wires were connected to ports within the autoclave which fed to the outside of the autoclave to connect to the AC conditioner module.

\section{A.1.1 Background}

The LVDT; consist of a small rod that slides within a cylindrical housing. The rod itself is nonmagnetic except for the tip which consists of a nickel-iron core. The housing has one primary solenoid and two secondary solenoids. Excitation voltage is applied to the primary coils which induces a voltage in the secondary coils (connected in series opposition). The secondary coils provide the AC signal that is transmitted to the conditioner module. When the core is in the "null" position, the induced voltages cancel and the output voltage is zero (when calibrated with the conditioner module). However, as the core displaces relative to the coils, the induced voltage will increase in one of the secondary coils and decrease in the other. The magnitude of the net voltage is proportional to the displacement of the rod. Phase polarity is referenced with the excitation voltage which determines the displacement direction. This is transmitted to the conditioner module by sensor wires connected to the positive and negative terminals of the primary solenoid. The AC conditioner module is necessary to distinguish between positive and negative displacements and filter out residual null voltage effects [1, 14, 15].

\section{A.1.2 AC conditioner calibration}

The LVDT conditioner was calibrated prior to experimentation according to the calibration step outlined in the 5M30 AC LVDT Conditioner product manual [14]. Calibration was performed such that a $6 \mathrm{~V}$ 


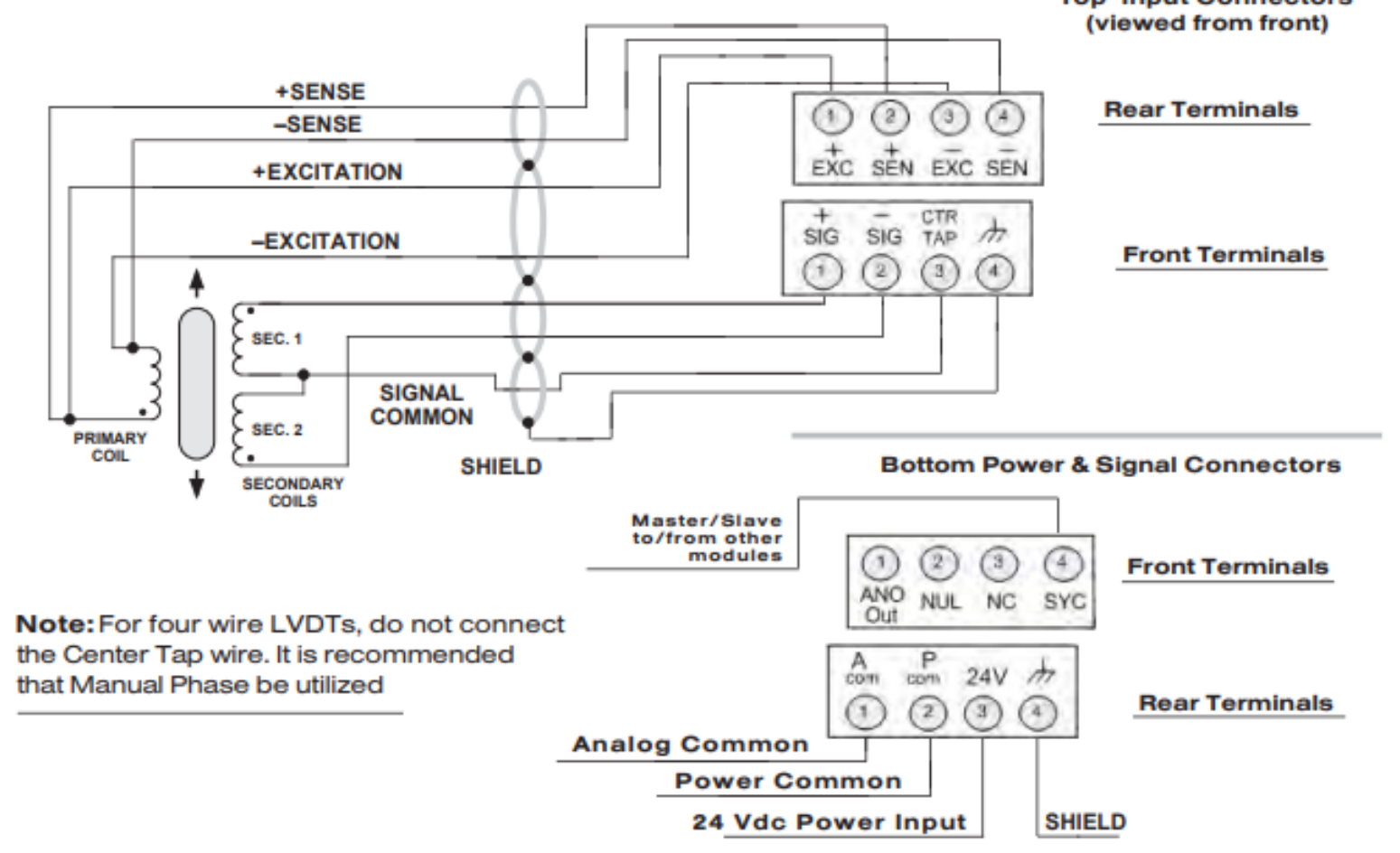

Figure A.1: LVDT wiring to conditioner module. Taken from [14].

output signal corresponded to 1 in $(25.4 \mathrm{~mm})$ of displacement. The switch states on the conditioner module were as follows:

- Switch 1 - Mode: Voltage

- Switch 2 - Volts: $5.0 \mathrm{Vdc}$

- Switch 3 - Current: Set to $4 \mathrm{~mA}$ but NA since mode is voltage

- Switch 4 - Filter: $100 \mathrm{~Hz}$

- Switch 5 - Filter: $100 \mathrm{~Hz}$

- Switch 6 - Sync: Master for LVDT 1 and Slave for LVDT 2

- Switch 7 - Zero Adj.: Normal

- Switch 8 - Range: Low (160-1600 mV/V)

- Switch 9 - Phase: Automatic

The schematic used for wiring is shown in Figure A.1. 


\section{A.2 In-core pressure sensors}

The in-core pressure sensors are developed by Convergent Manufacturing Technologies [3]. They are piezoresistive MEMS pressure sensors utilizing a Wheatstone bridge setup. Specs are provided below. Owing to proprietary reasons, limited information is available.

- Bridge resistance: $3.4 \mathrm{k} \Omega( \pm 0.4 \mathrm{k} \Omega)$

- Operating pressure: 7 bar

- Operating temperature: $-40^{\circ} \mathrm{C}$ to $180^{\circ} \mathrm{C}$

- Repeatability: $\pm 0.2 \%$ F.S.

The sensors comprise the pressure transducer with four wires corresponding to excitation voltage, ground, positive output voltage, and negative output voltage. Excitation voltage is 5 volts. Output voltage corresponds to the level of pressure application and is measured across the Wheatstone bridge. The output voltage is temperature dependent and is correlated to pressure through a specific transfer function. Each sensor is individually calibrated by Convergent Manufacturing Technologies.

\section{A.3 Autoclavable camera}

The in-autoclave camera was developed by Convergent Manufacturing Technologies [3]. Owing to proprietary reasons, limited information is available. The camera was mounted on an 80/20 aluminum frame to capture in-situ video footage of the core movement process. Video data was passed through a port within the autoclave directly to an external Laptop. The free software, VideoVelocity, developed by CandyLabs [2] was used to record and export video footage and real time images. The images were later compiled on MATLAB and correlated with the sensor data to produce synchronized, real time video footage with sensory display. An example of the synchronized footage is provided in Figure A.2.
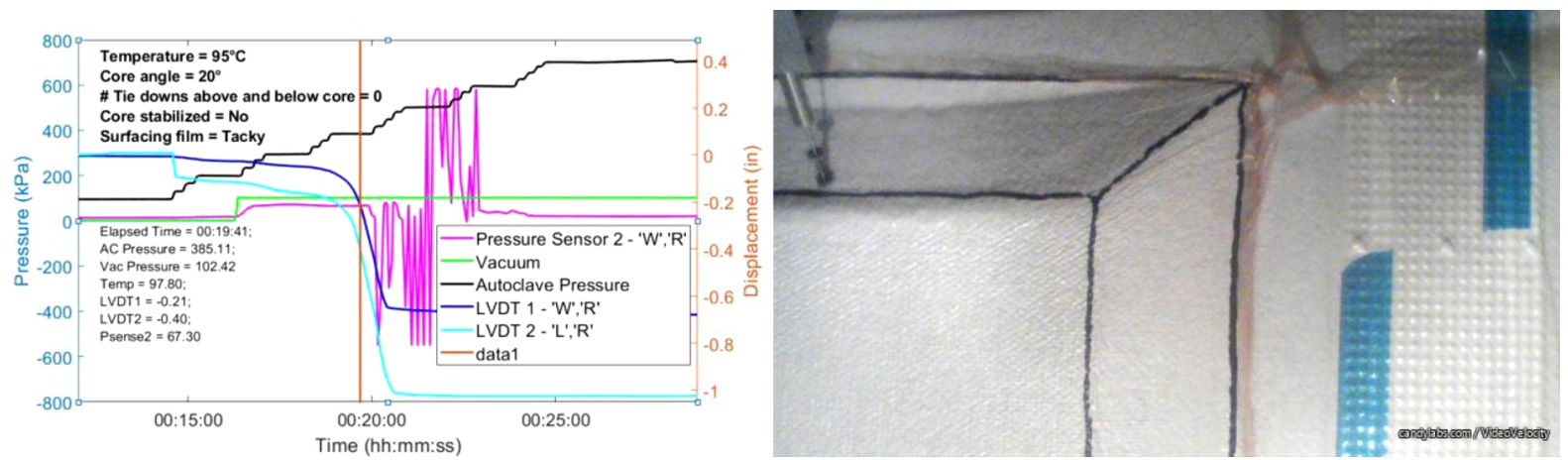

Figure A.2: Video footage synchronized with in-situ sensor data. $95^{\circ} \mathrm{C}$ experiment. 


\section{Appendix B}

\section{Appendix B Additional Post-processing Images}

\section{B.1 Post-processed panels}

Images of each of the panels post processing are provided the below. They are organized according to their experimental group. That is, the top row represents the "Effect of Temperature" tests, the middle row "Effect of Pressure", and the bottom row "Effect of Layup". 


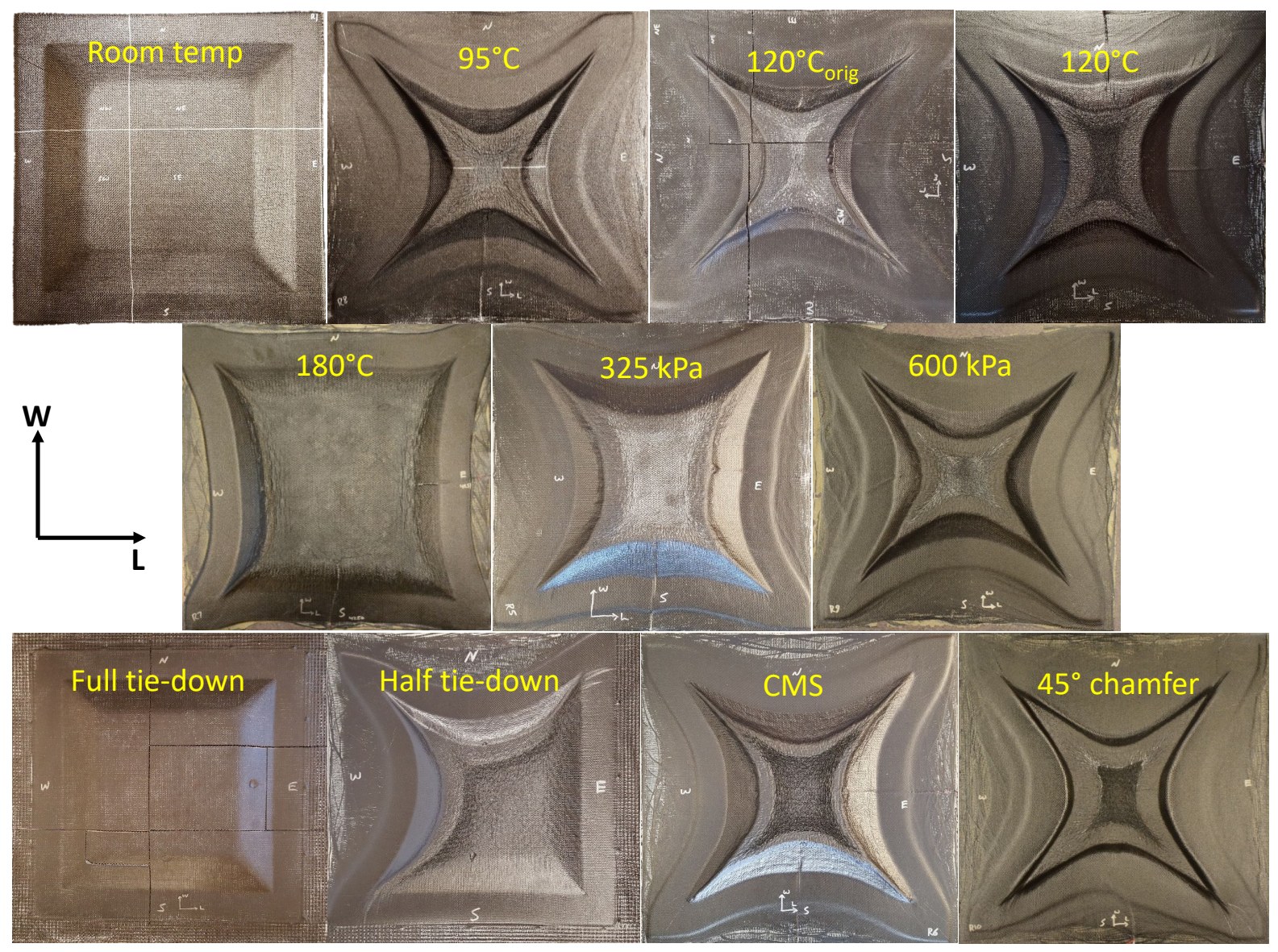

Figure B.1: Top-down view of processed panels.

\section{B.2 Slope gradient maps}

Slope gradient maps are provided below for each sample. Unfortunately, the room temperature sample was not scanned on the CMM. As such, no slope gradient map exists for this sample. 


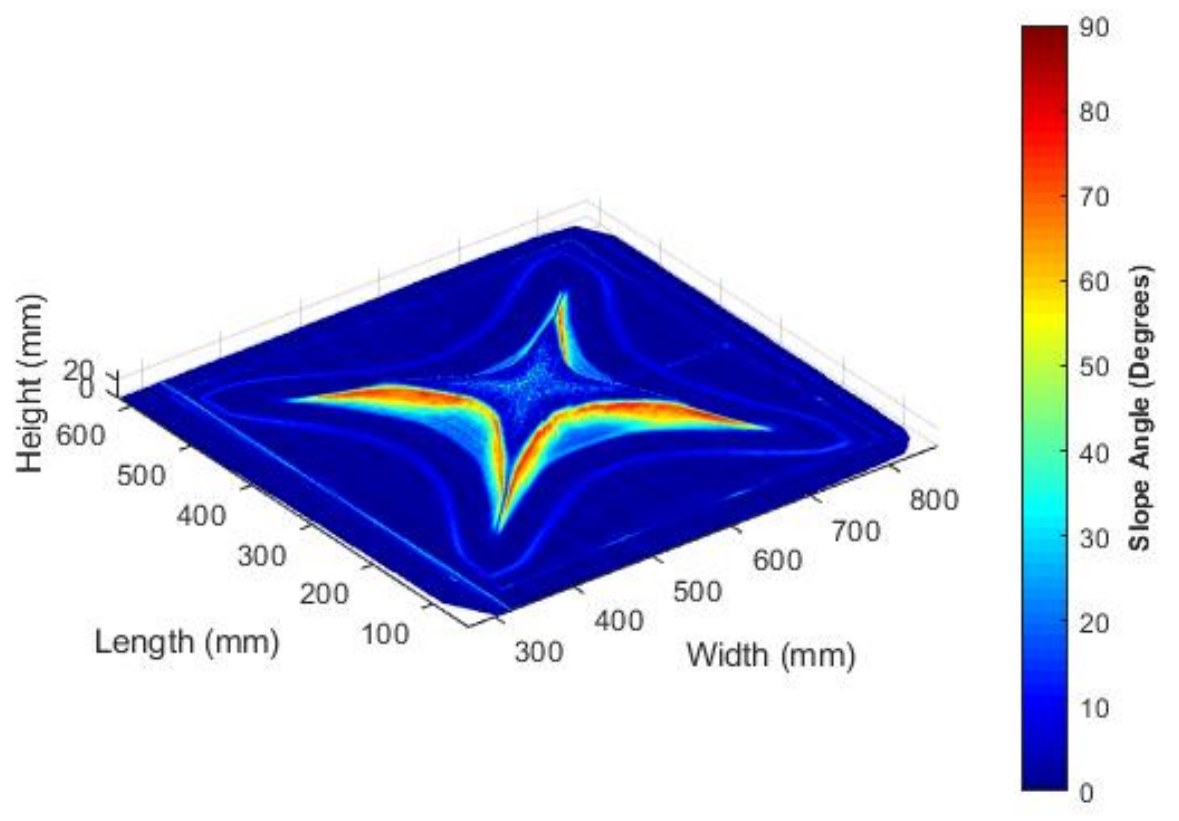

Figure B.2: Slope gradient map for $95^{\circ} \mathrm{C}$ sample. Although not shown, the colour bar applies to each of the following images. Noise is seen in some of the samples which can be disregarded.

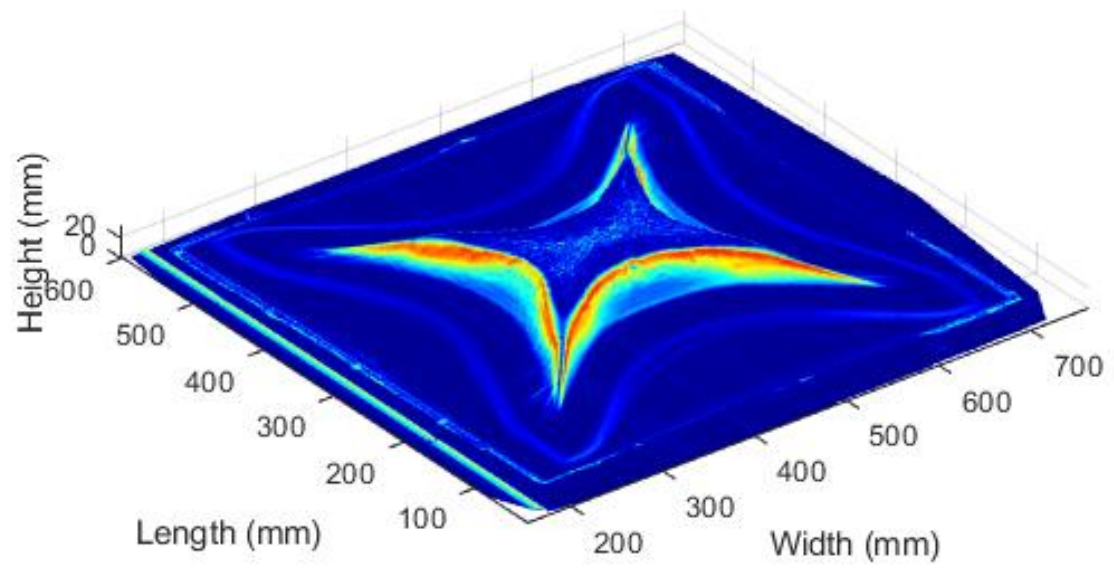

Figure B.3: Slope gradient map for $120^{\circ} C_{\text {orig }}$ sample. 


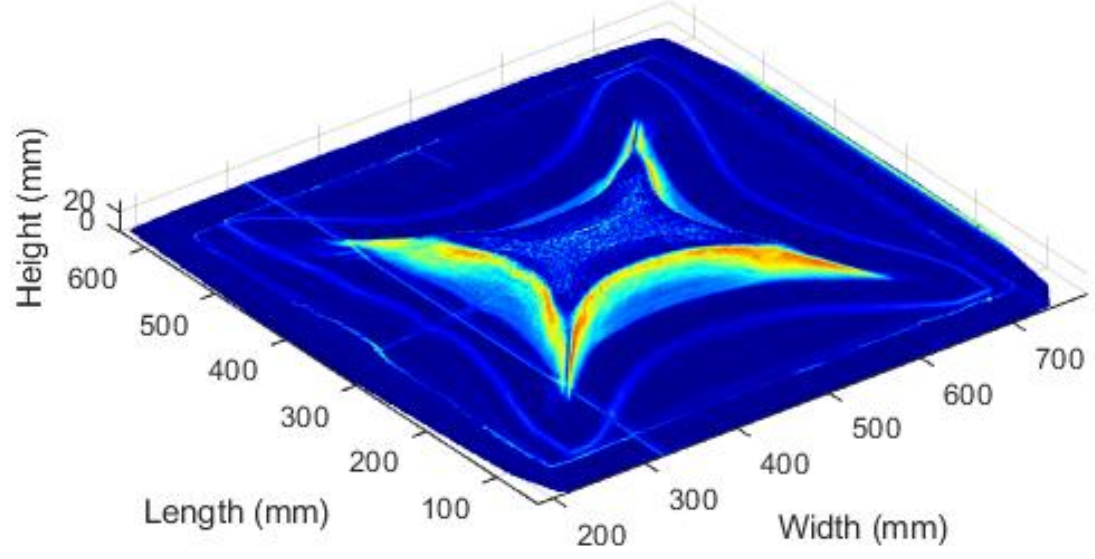

Figure B.4: Slope gradient map for $120^{\circ} \mathrm{C}$ sample.

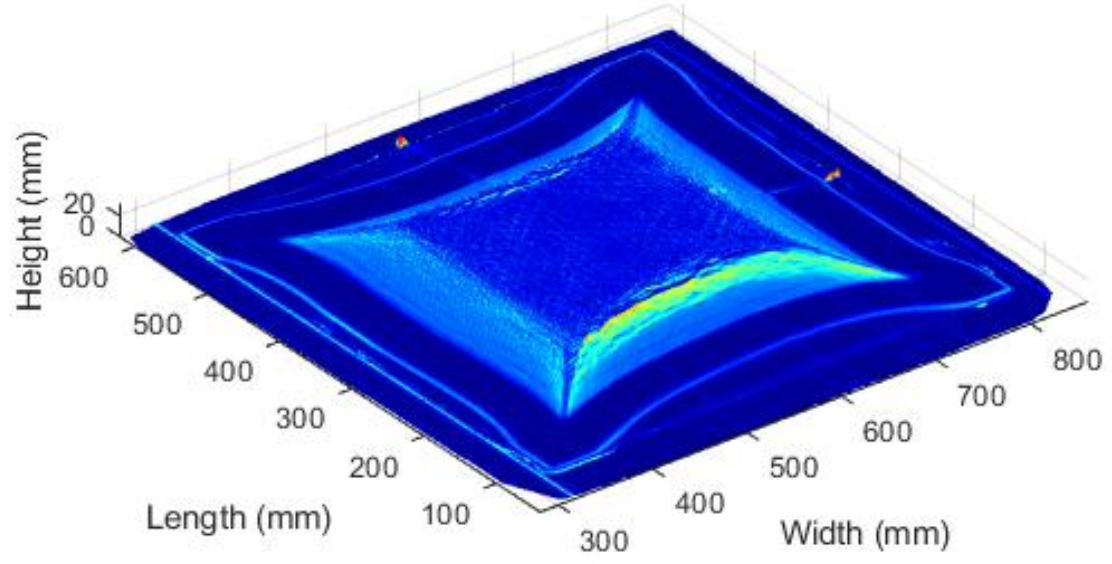

Figure B.5: Slope gradient map for $180^{\circ} \mathrm{C}$ sample. 


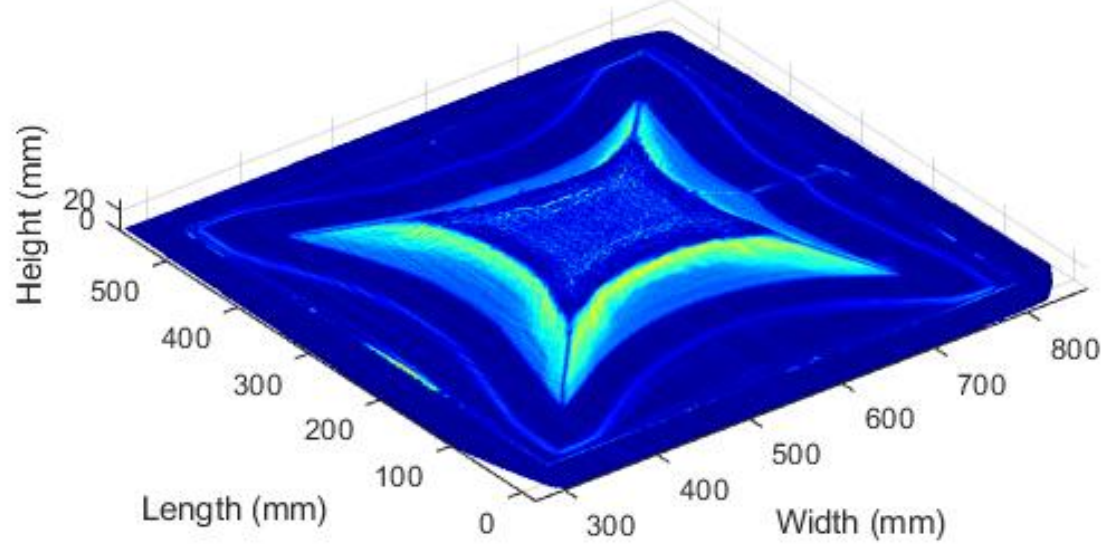

Figure B.6: Slope gradient map for $325 \mathrm{kPa}$ sample.

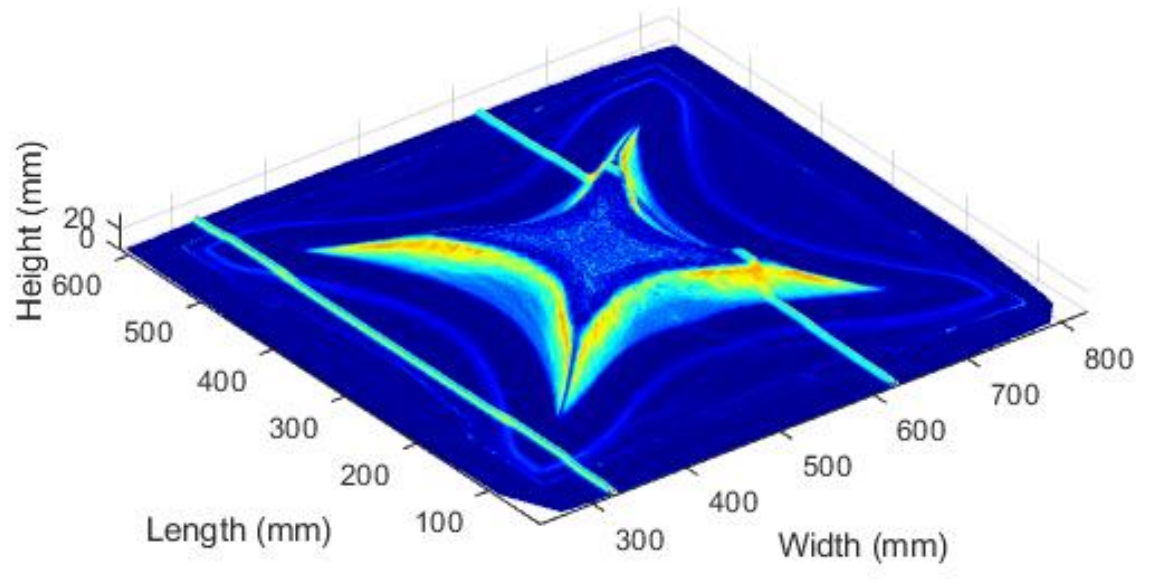

Figure B.7: Slope gradient map for $600 \mathrm{kPa}$ sample. 


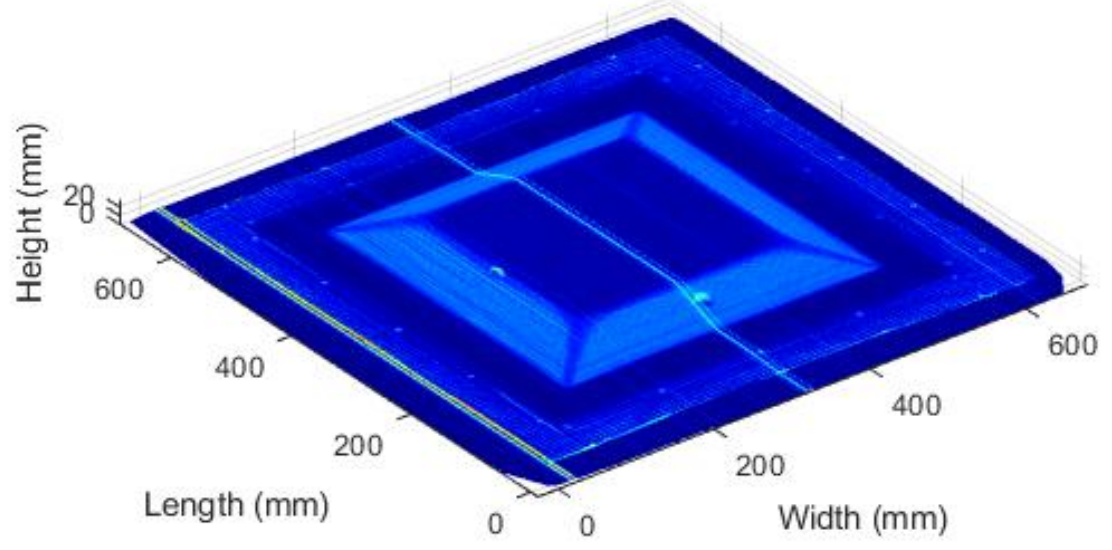

Figure B.8: Slope gradient map for full tie-down sample.

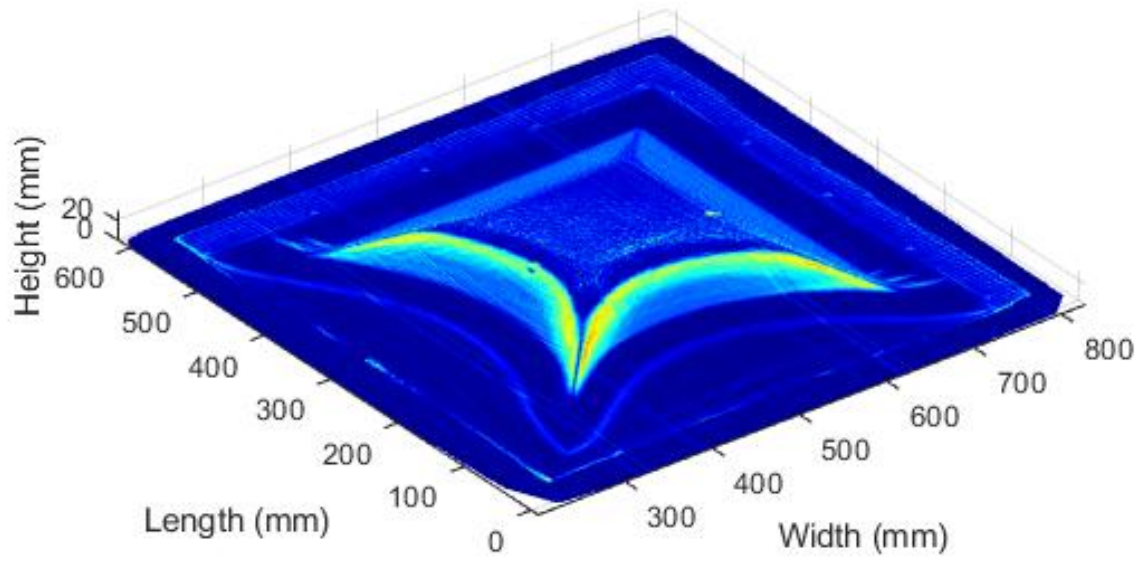

Figure B.9: Slope gradient map for half tie-down sample. 


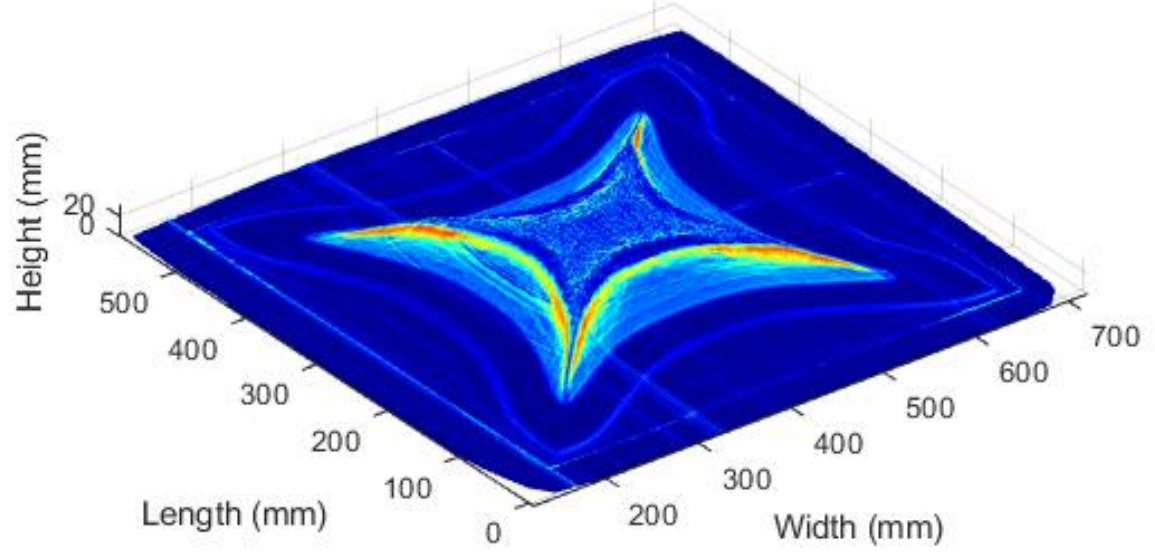

Figure B.10: Slope gradient map for CMS sample.

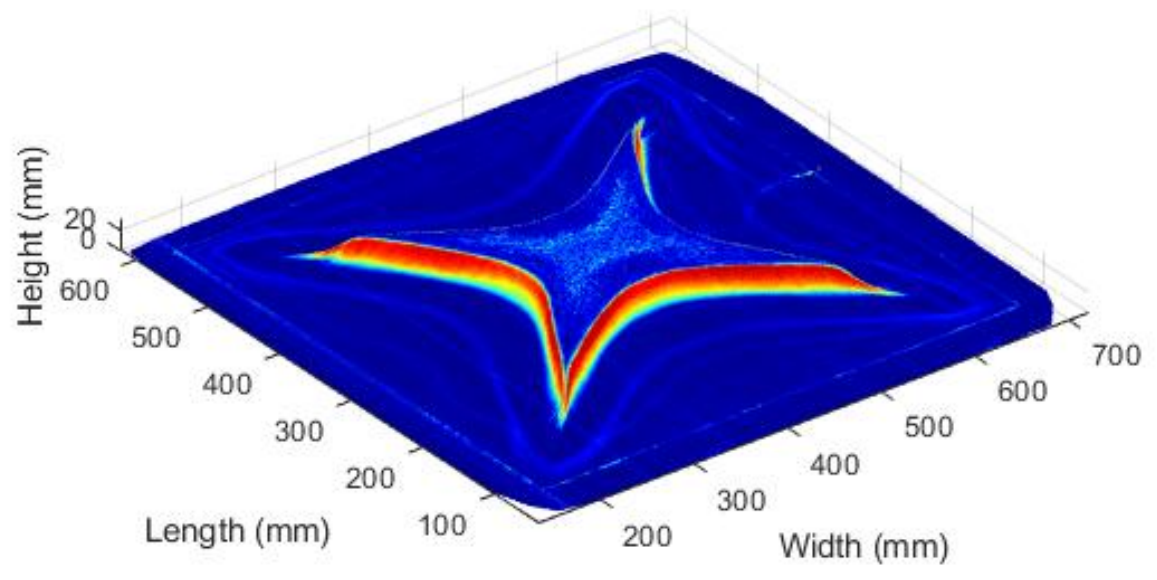

Figure B.11: Slope gradient map for $45^{\circ}$ chamfer sample. 


\section{B.3 Through-thickness section cuts}

Images of through-thickness section cuts in the $\mathrm{L}$ and $\mathrm{W}$ direction for each sample are provided below, including those shown previously. Crush features are not identified. 


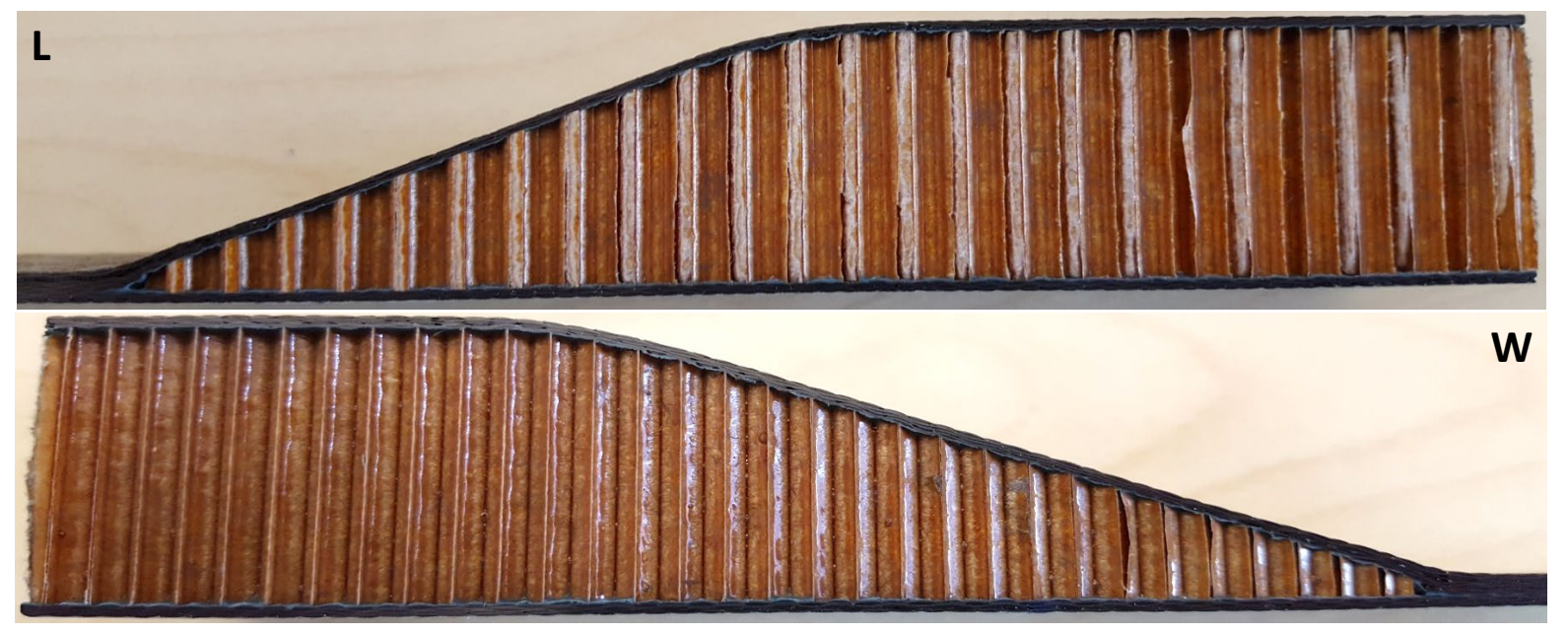

Figure B.12: Through-thickness section cut of room temperature sample.

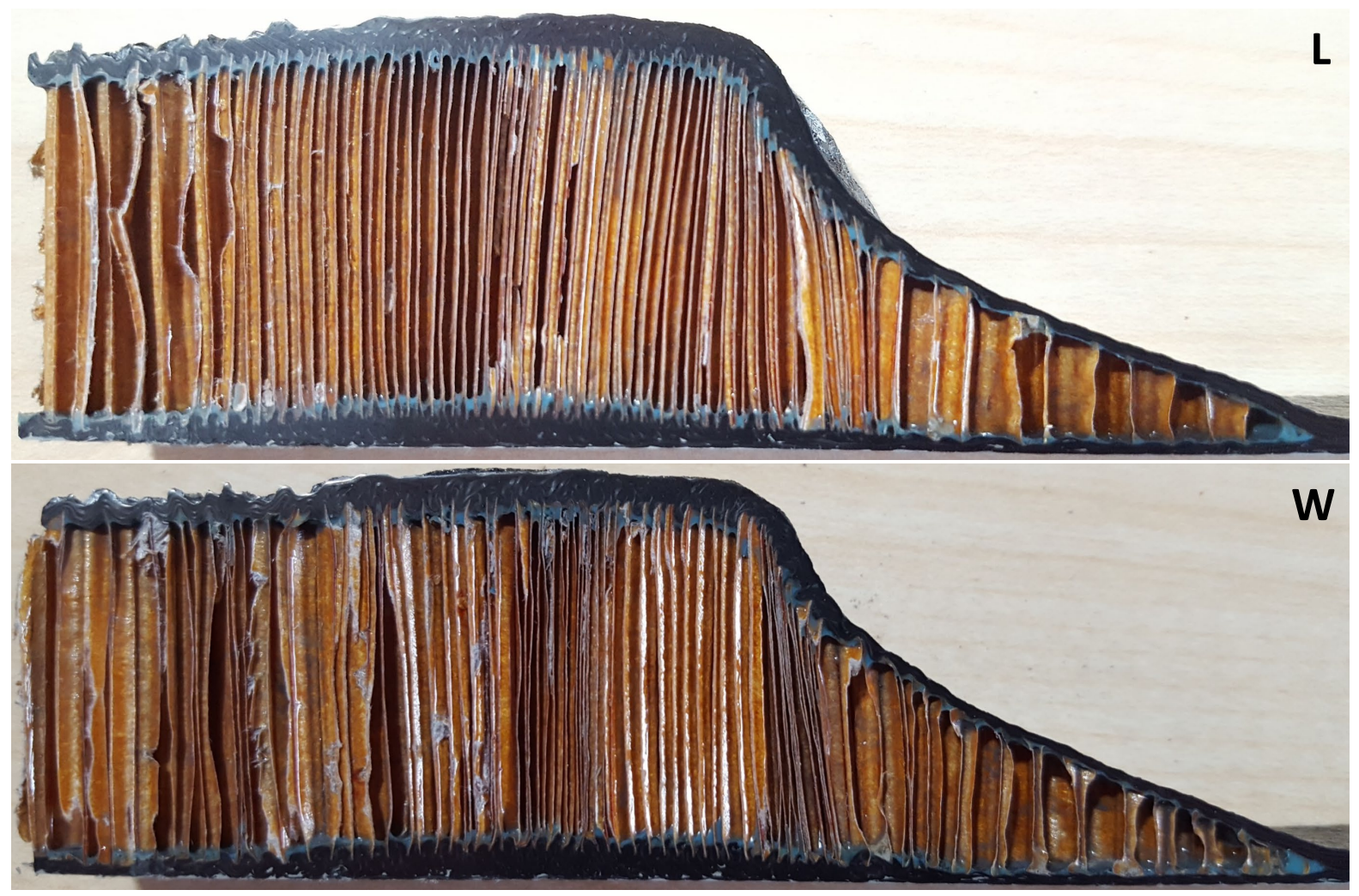

Figure B.13: Through-thickness section cut of $95^{\circ} \mathrm{C}$ sample. 

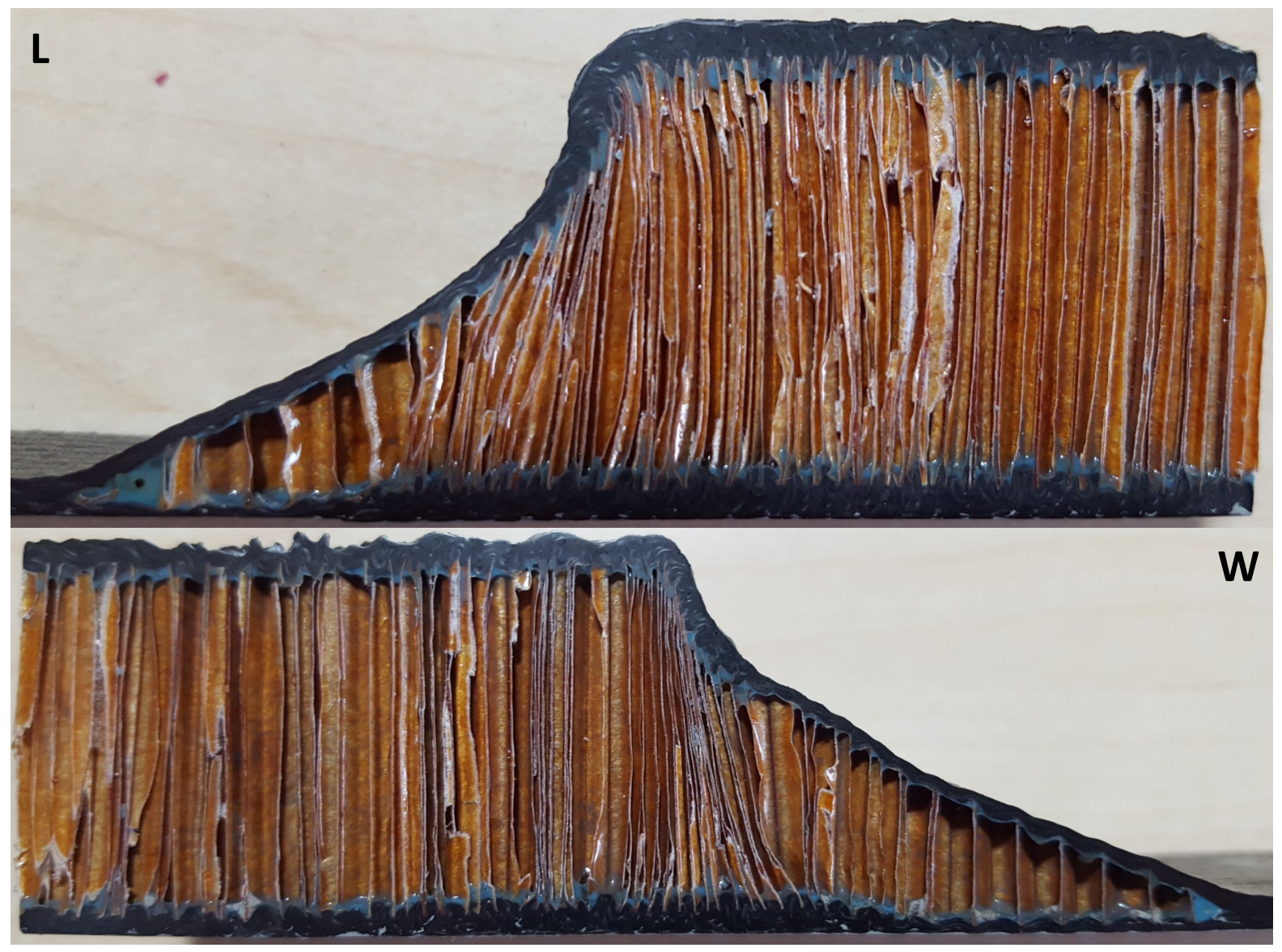

Figure B.14: Through-thickness section cut of $120^{\circ} \mathrm{C}_{\text {orig }}$ sample.
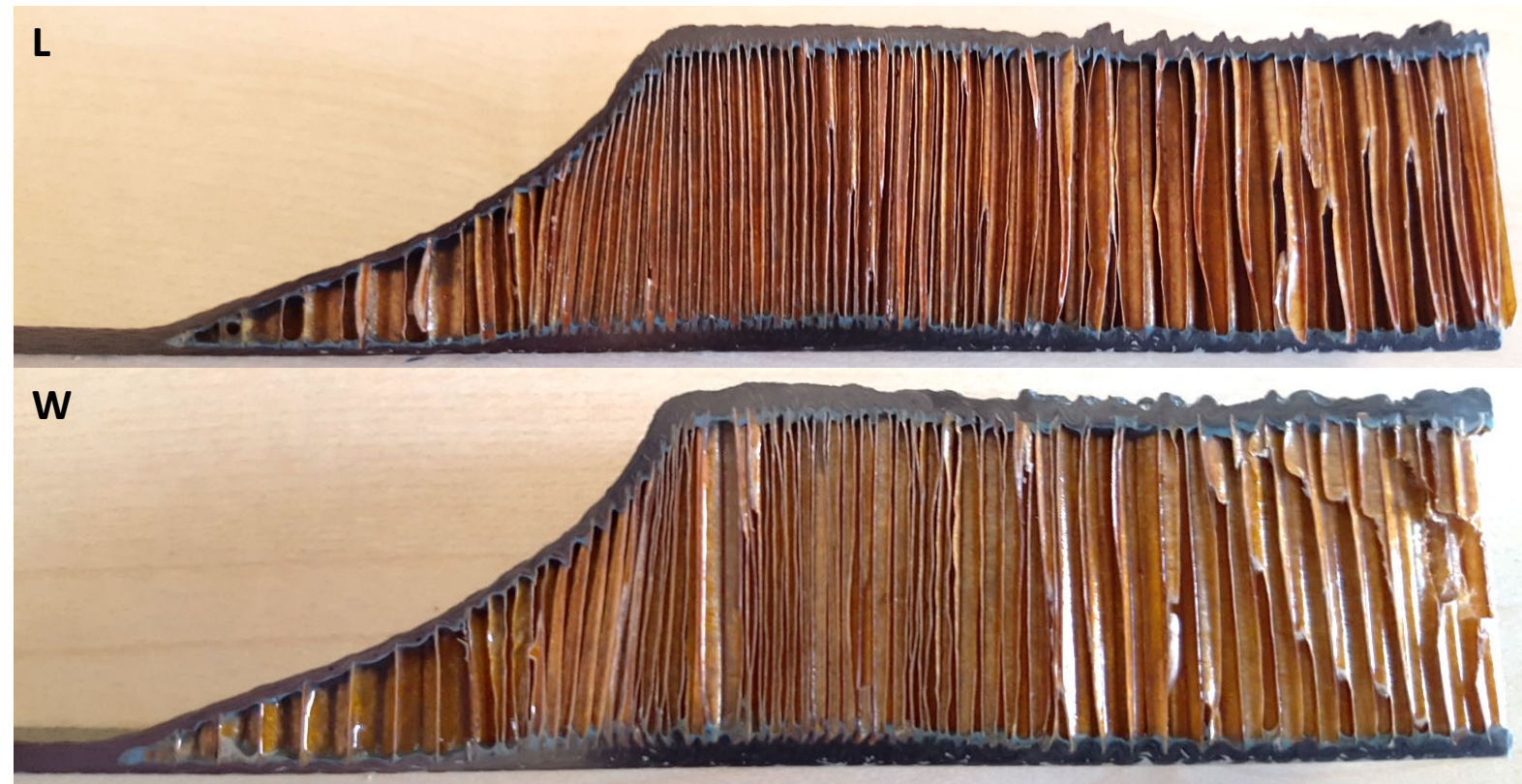

Figure B.15: Through-thickness section cut of $120^{\circ} \mathrm{C}$ sample. 

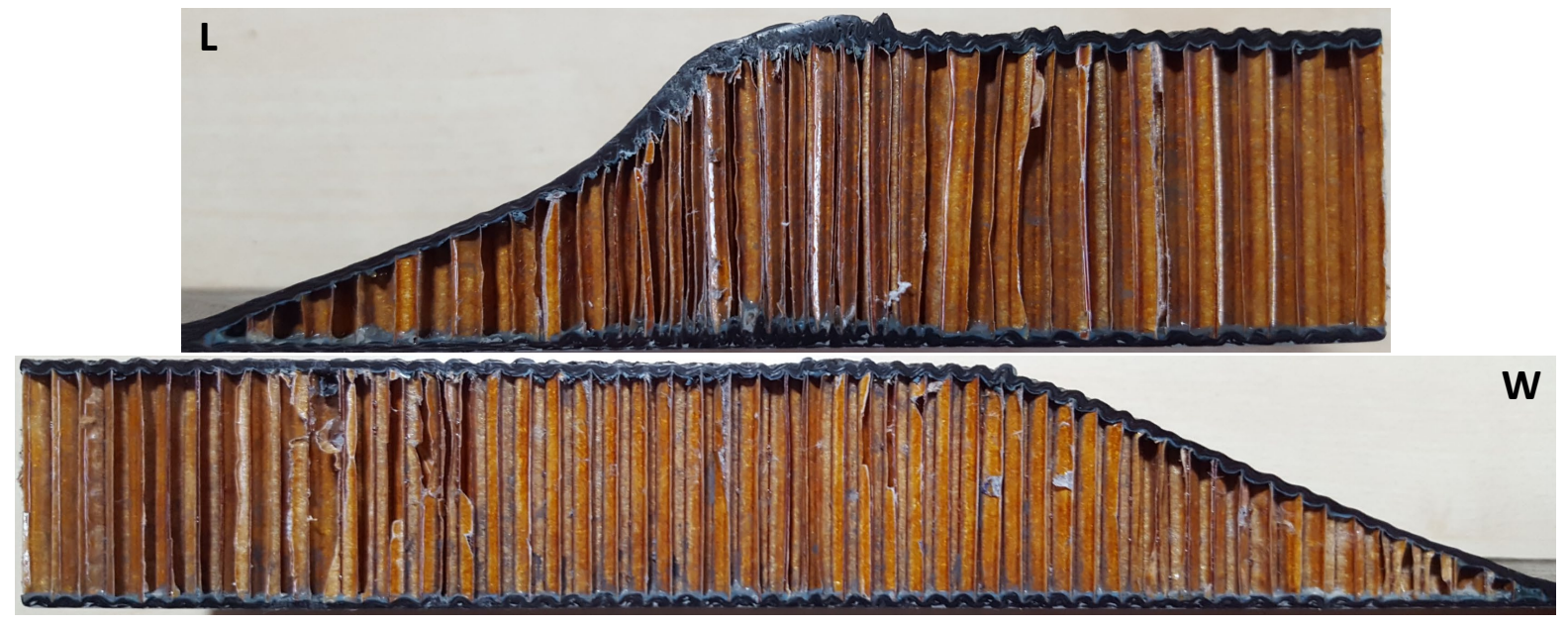

Figure B.16: Through-thickness section cut of $180^{\circ} \mathrm{C}$ sample.

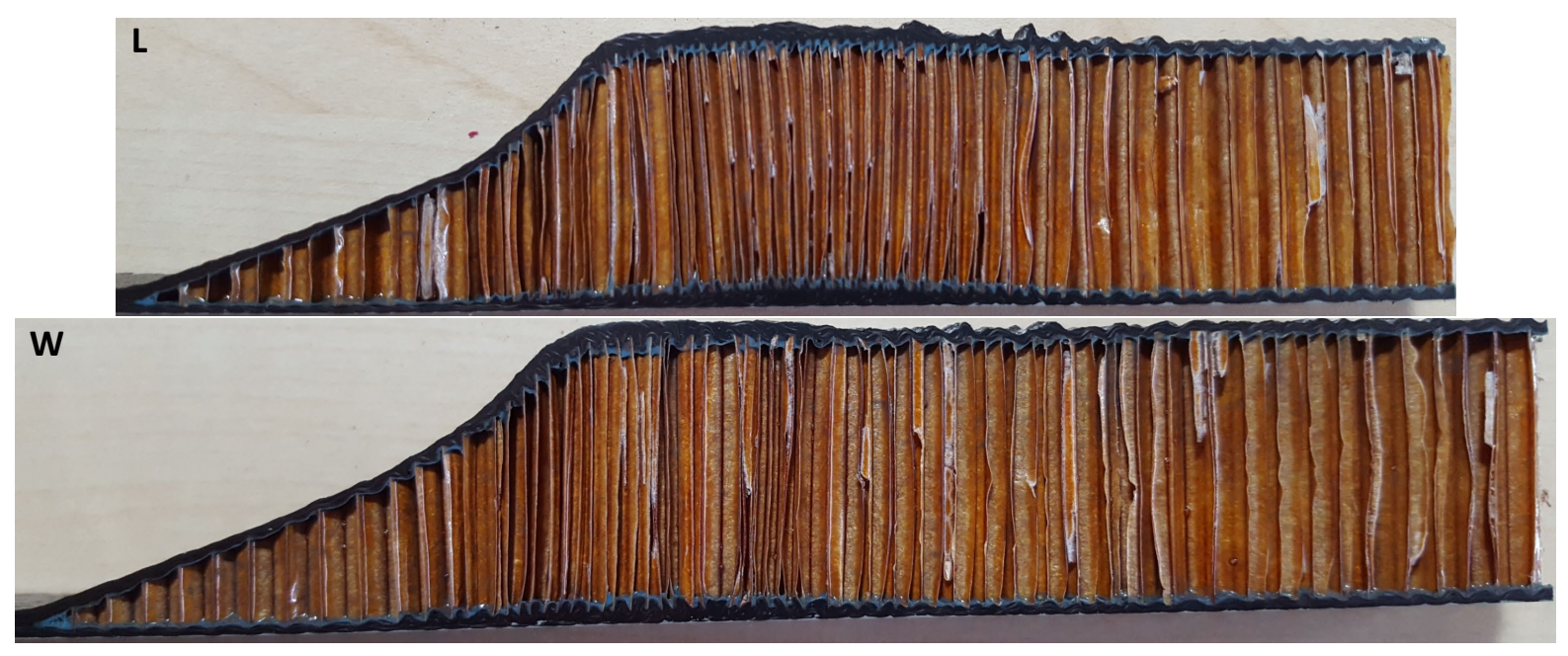

Figure B.17: Through-thickness section cut of $325 \mathrm{kPa}$ sample. 


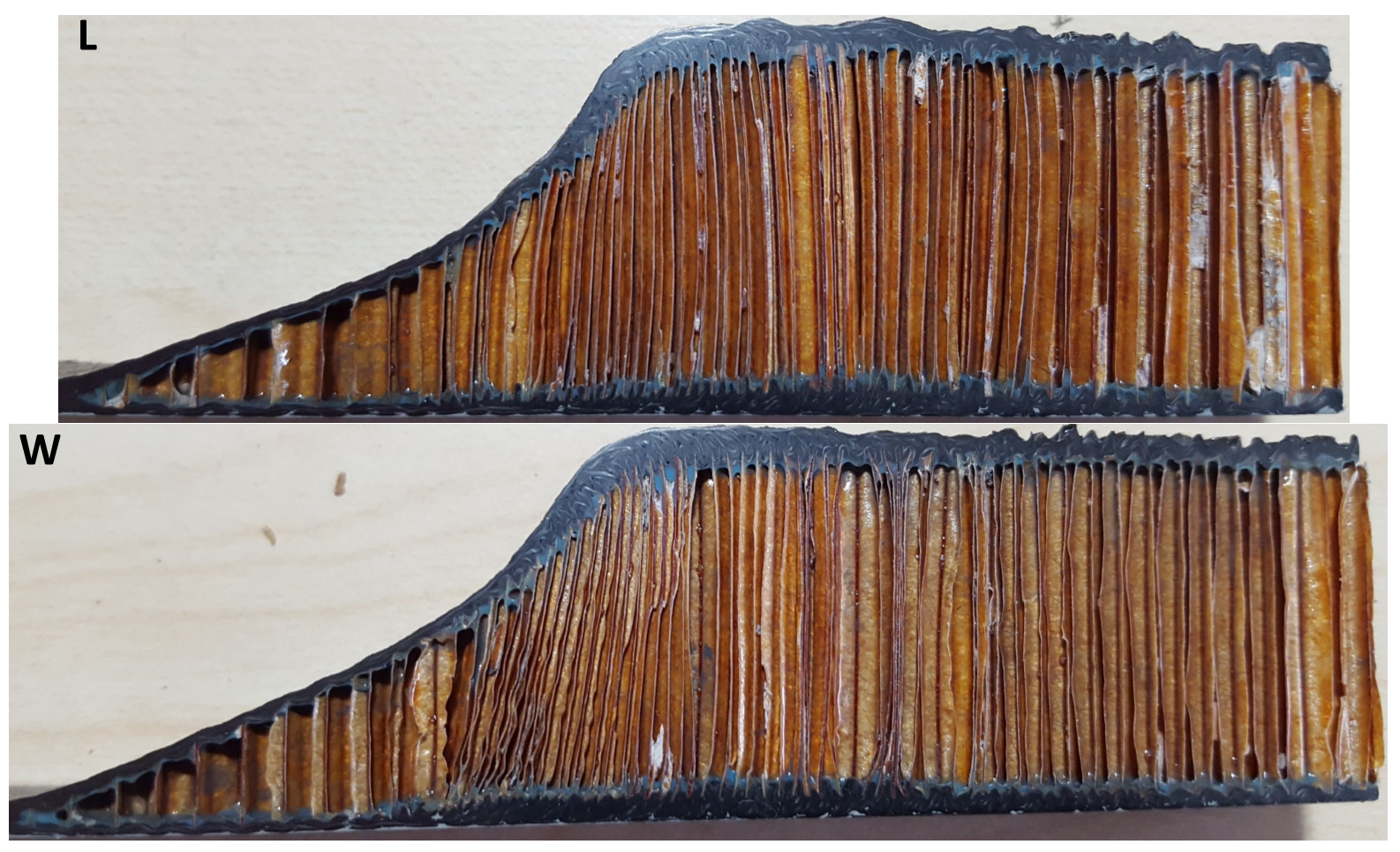

Figure B.18: Through-thickness section cut of $600 \mathrm{kPa}$ sample.
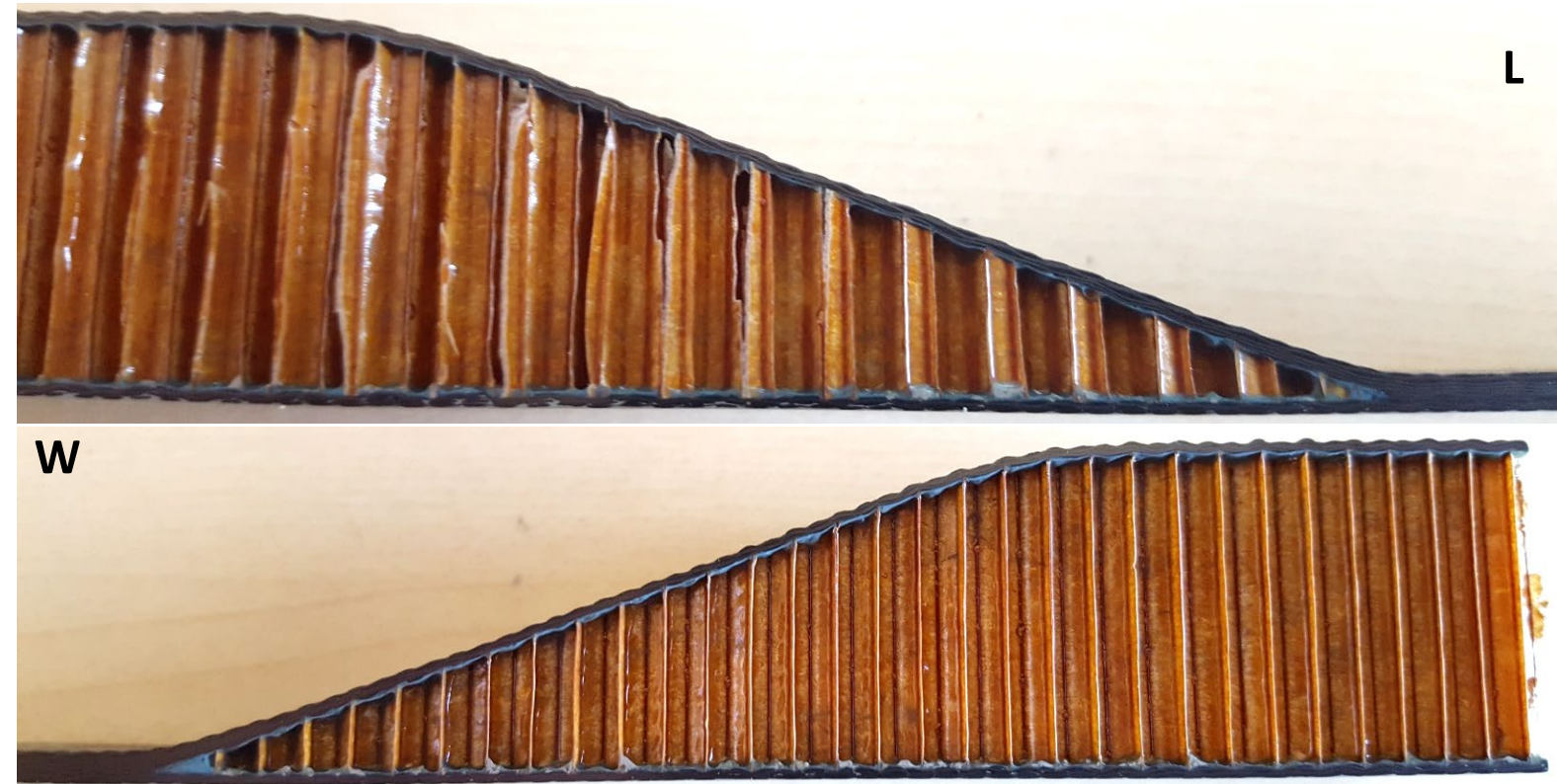

Figure B.19: Through-thickness section cut of full tie-down sample. 


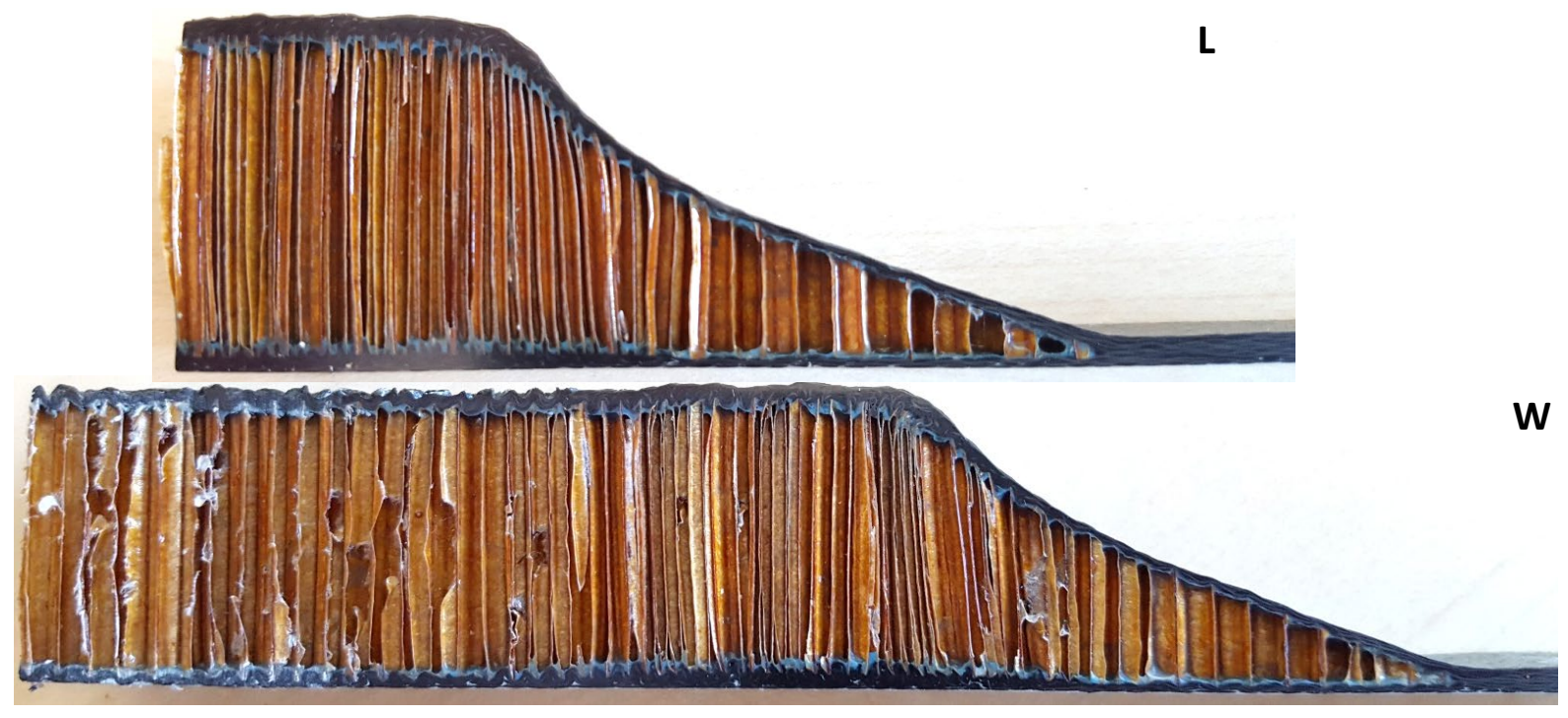

Figure B.20: Through-thickness section cut of half tie-down sample.

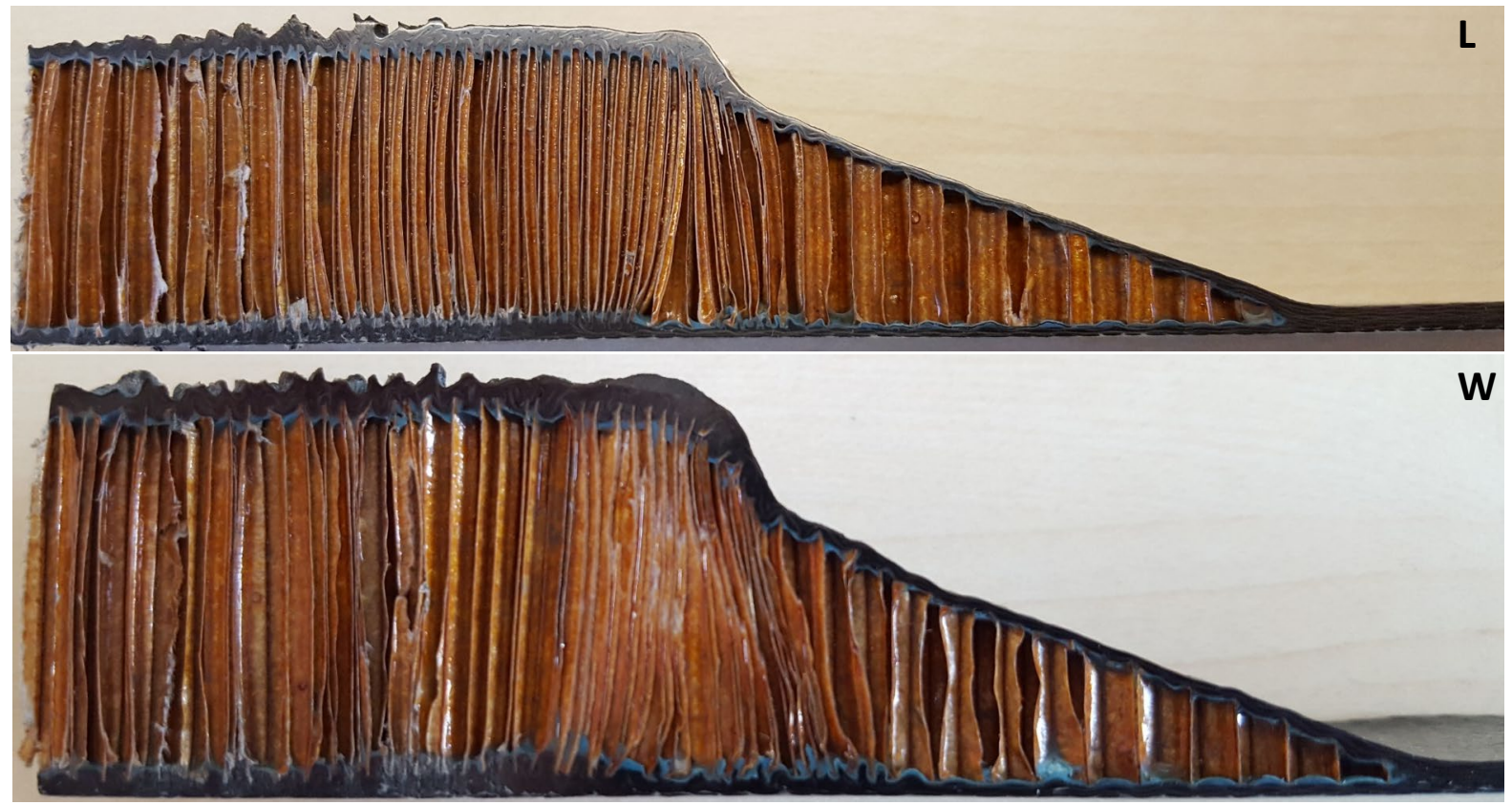

Figure B.21: Through-thickness section cut of CMS sample. 


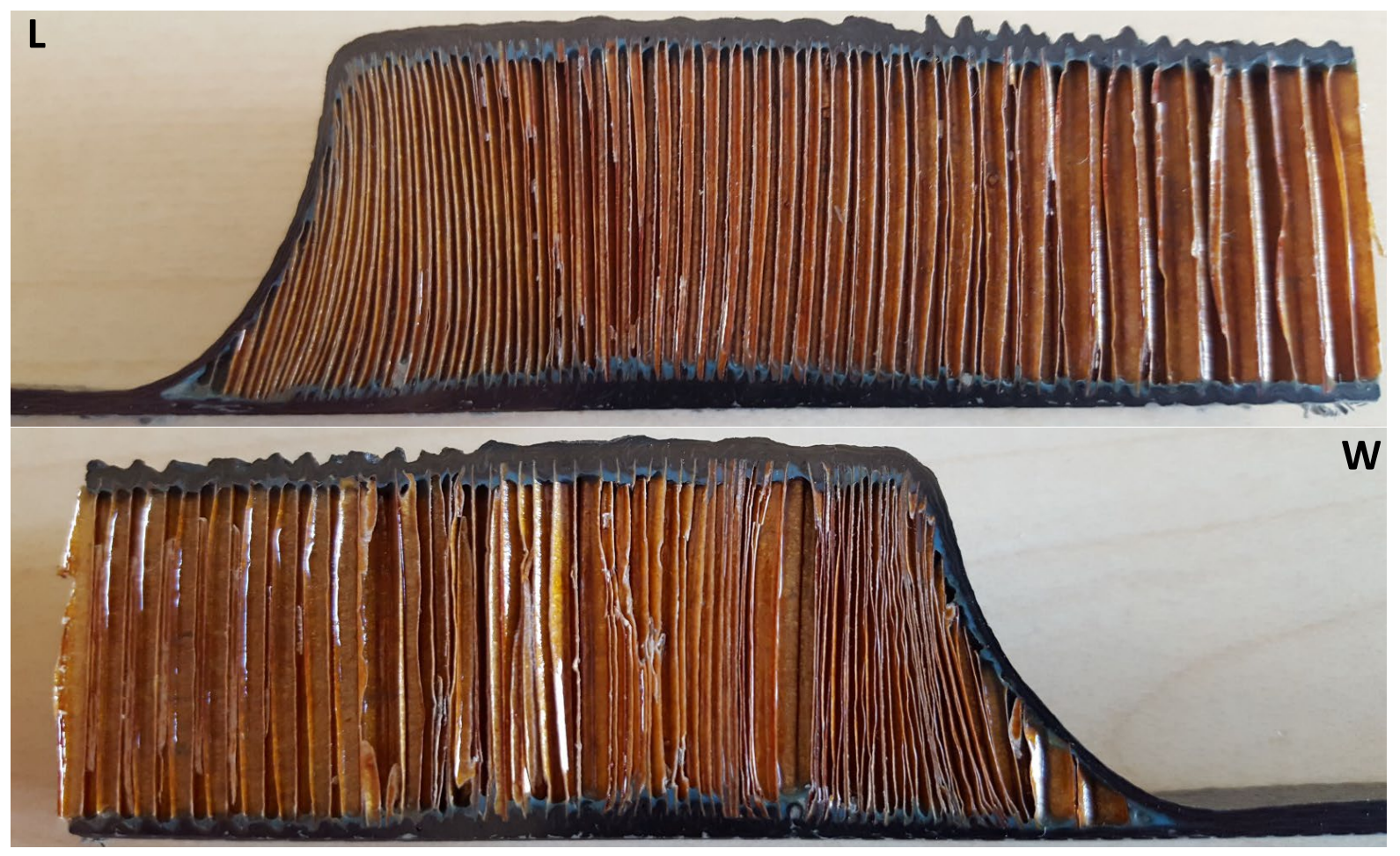

Figure B.22: Through-thickness section cut of $45^{\circ}$ chamfer sample. 


\section{B.4 In-plane section cuts}

The following section provides additional images of the in-plane section cuts made. Note that cuts were not made for each sample. All cuts made are displayed below including those previously shown.

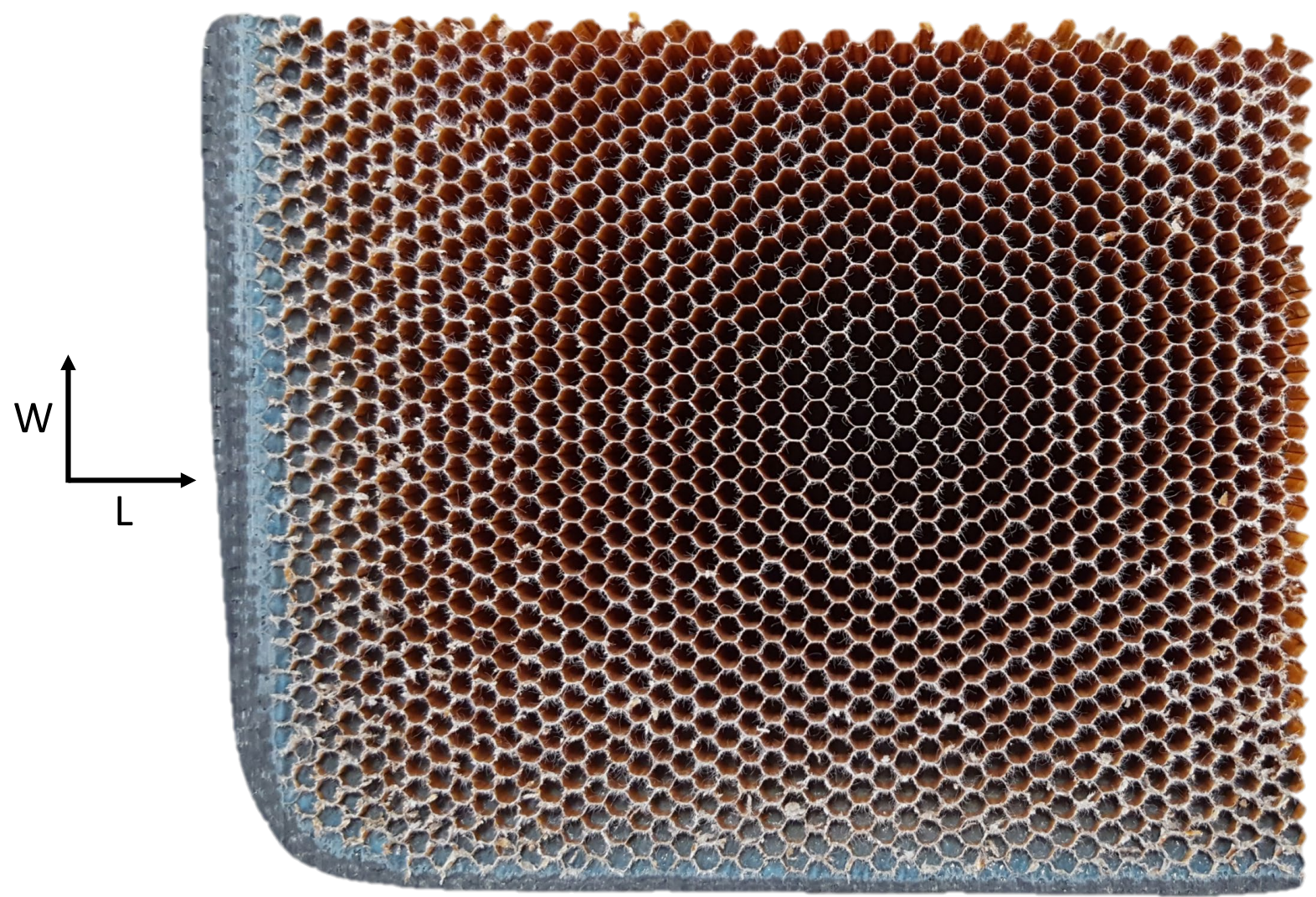

Figure B.23: In-plane section cut of room temperature sample. 


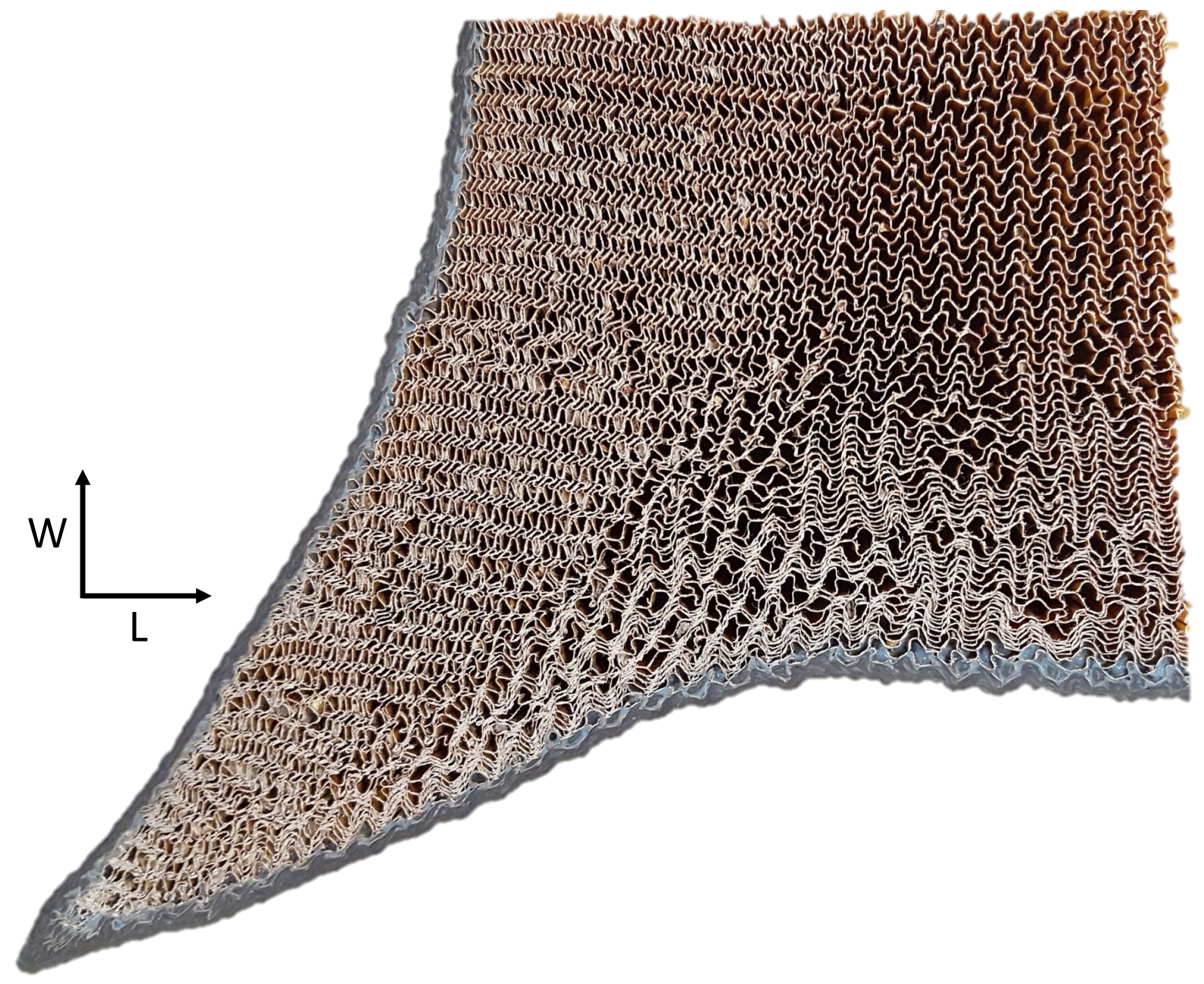

Figure B.24: In-plane section cut of $120^{\circ} \mathrm{C}$ sample. 


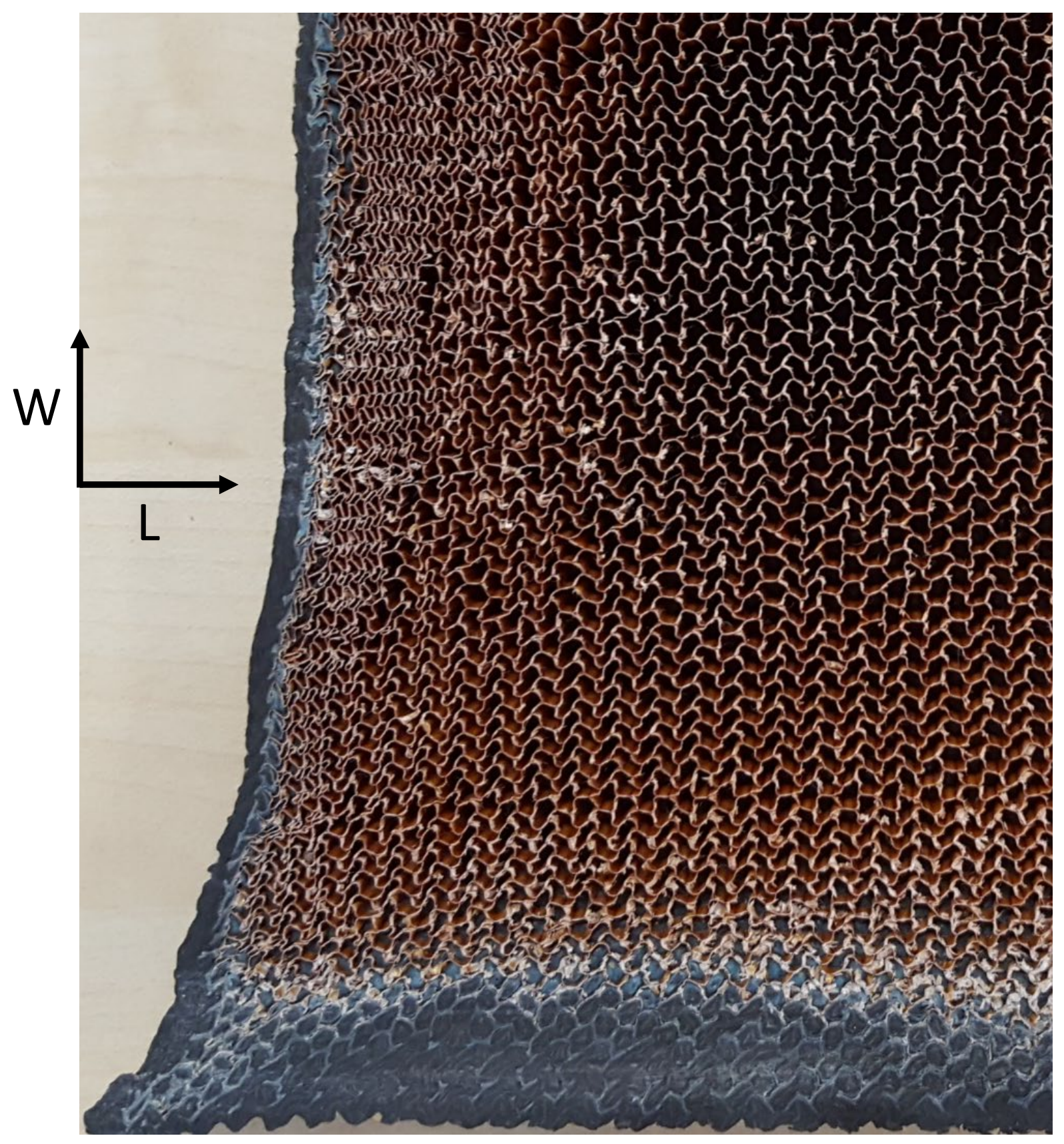

Figure B.25: In-plane section cut of $180^{\circ} \mathrm{C}$ sample. 


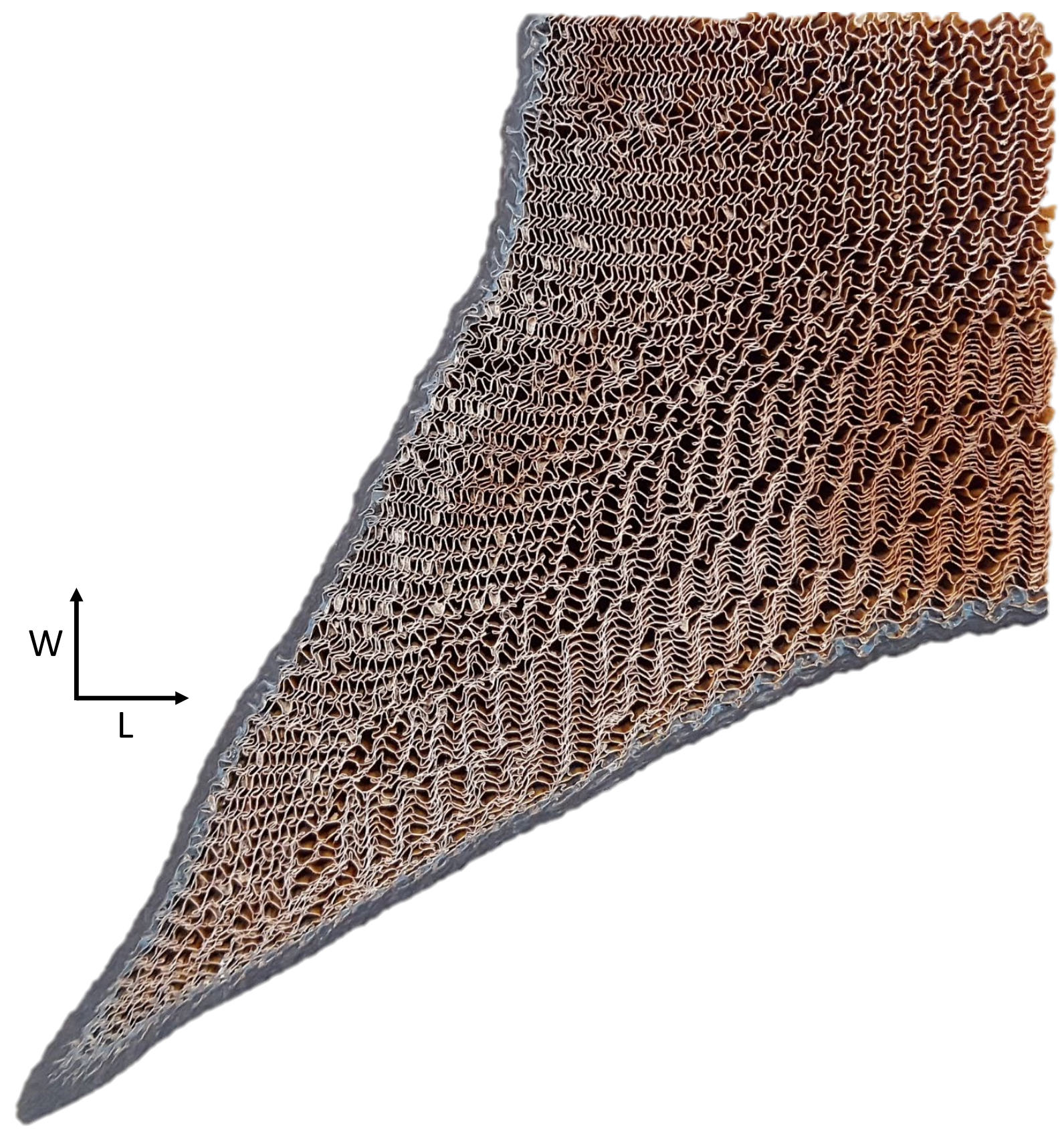

Figure B.26: In-plane section cut of $600 \mathrm{kPa}$ sample. 


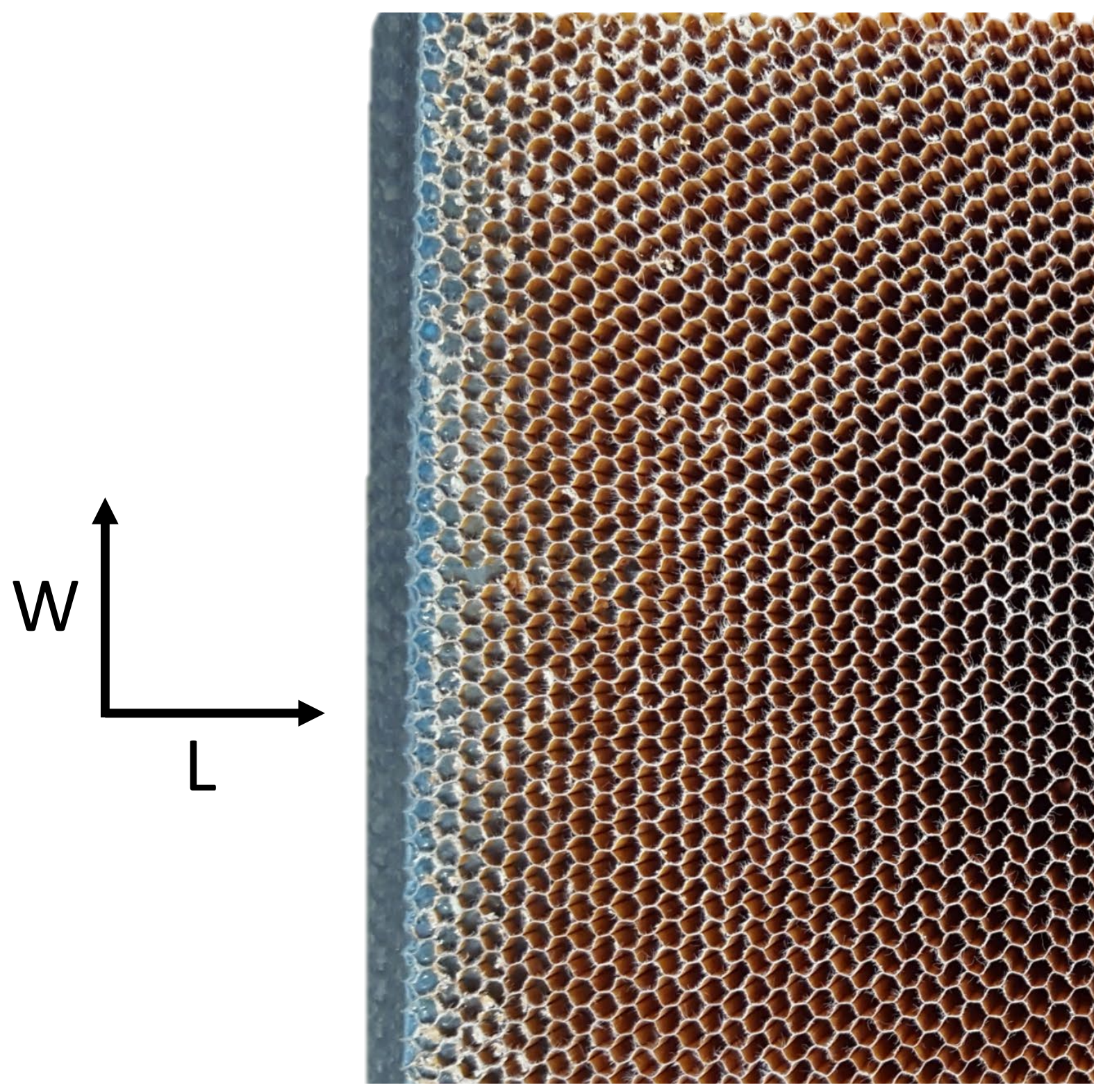

Figure B.27: In-plane section cut of full tie-down sample. 

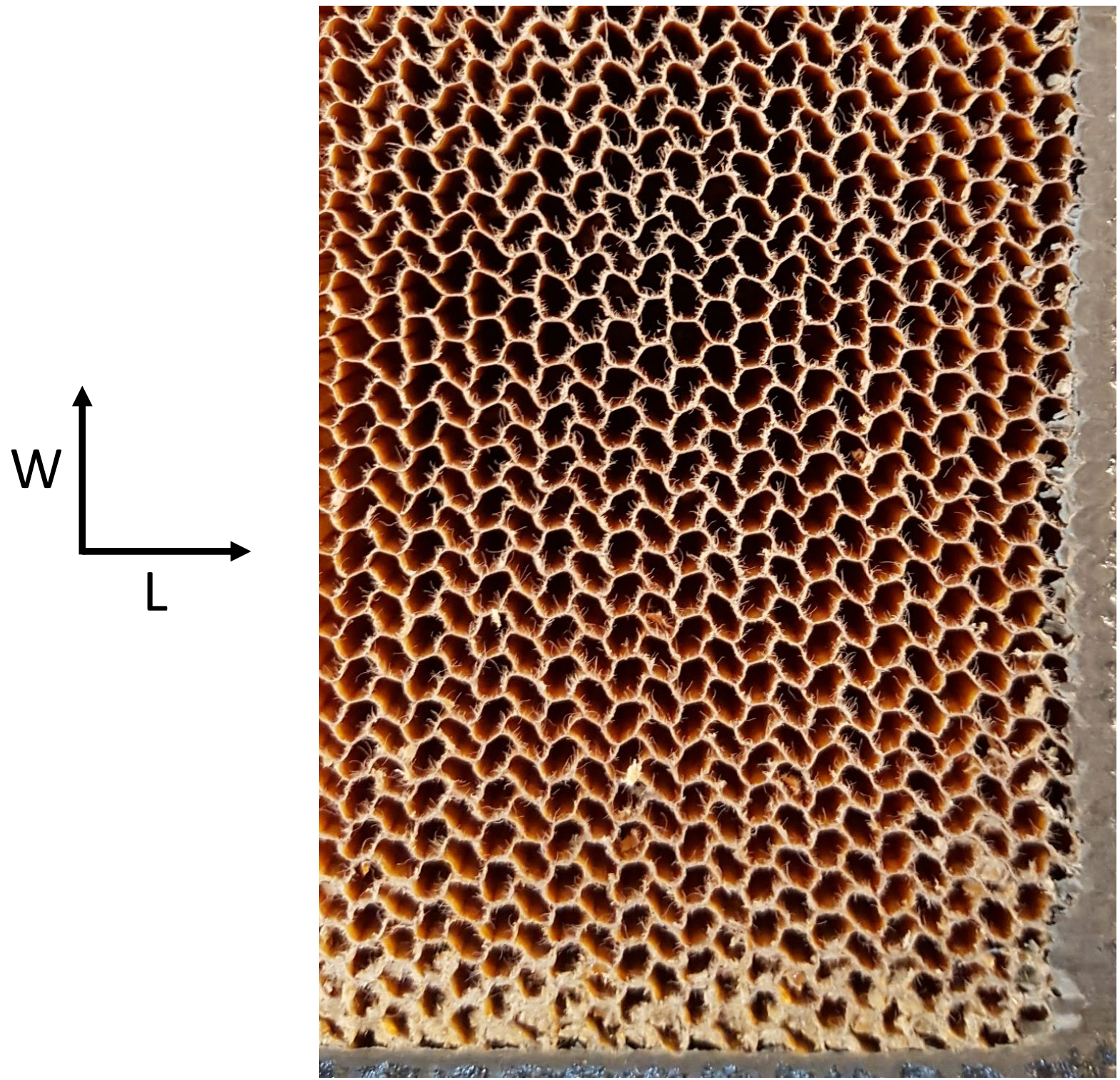

Figure B.28: In-plane section cut of half tie-down sample between two restrained edges. 


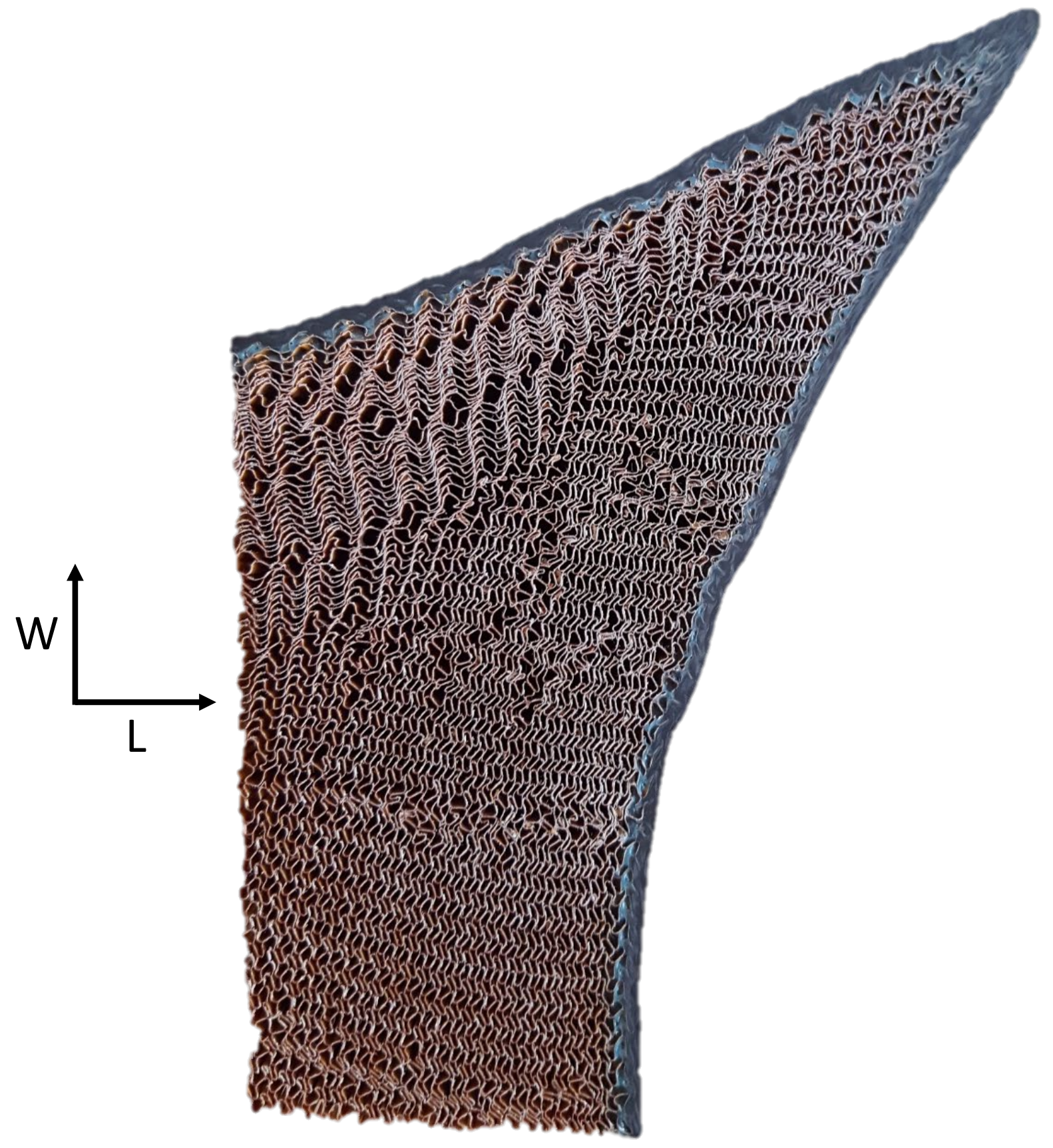

Figure B.29: In-plane section cut of half tie-down sample between two unrestrained edges. 


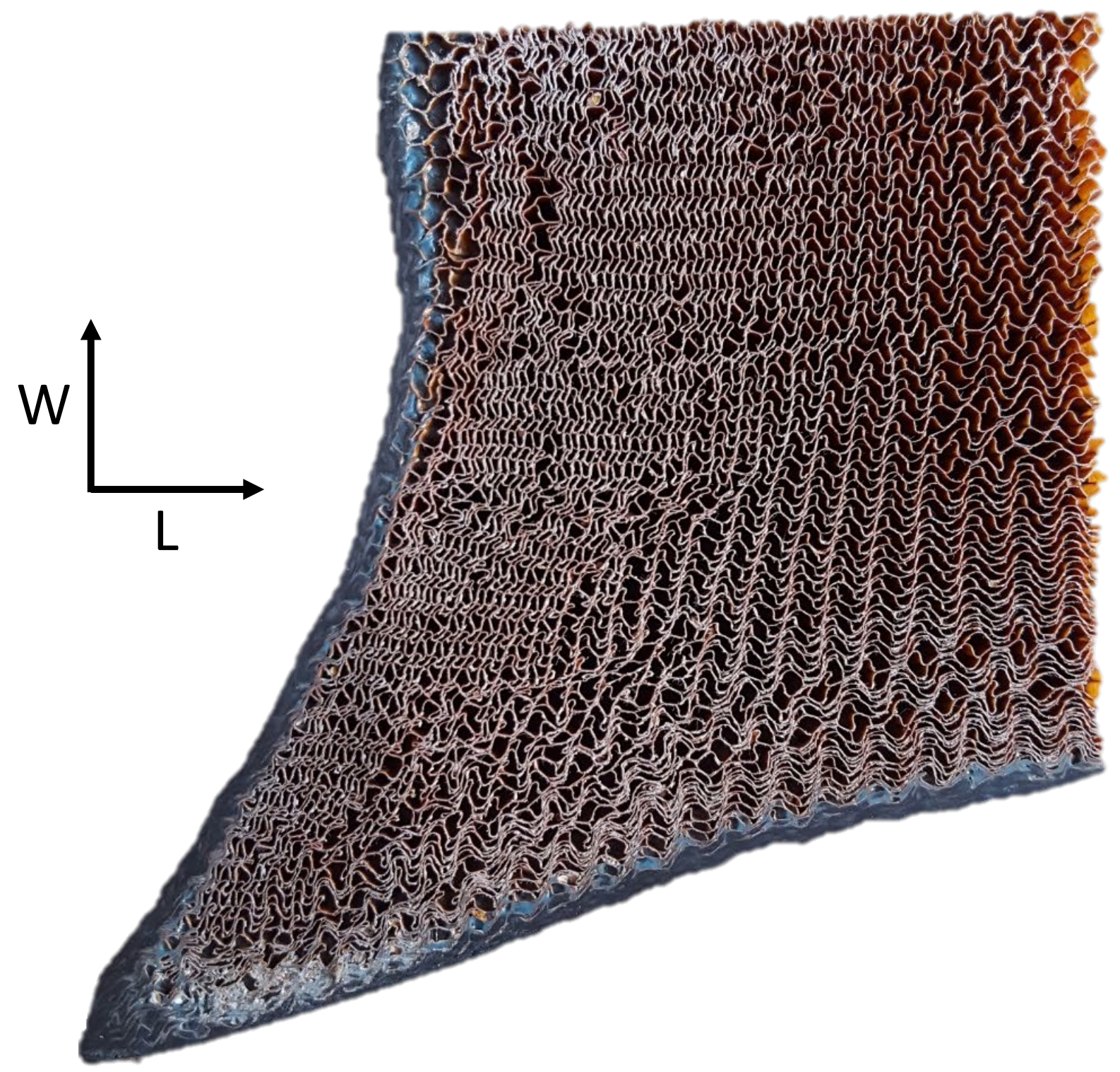

Figure B.30: In-plane section cut of CMS sample. 


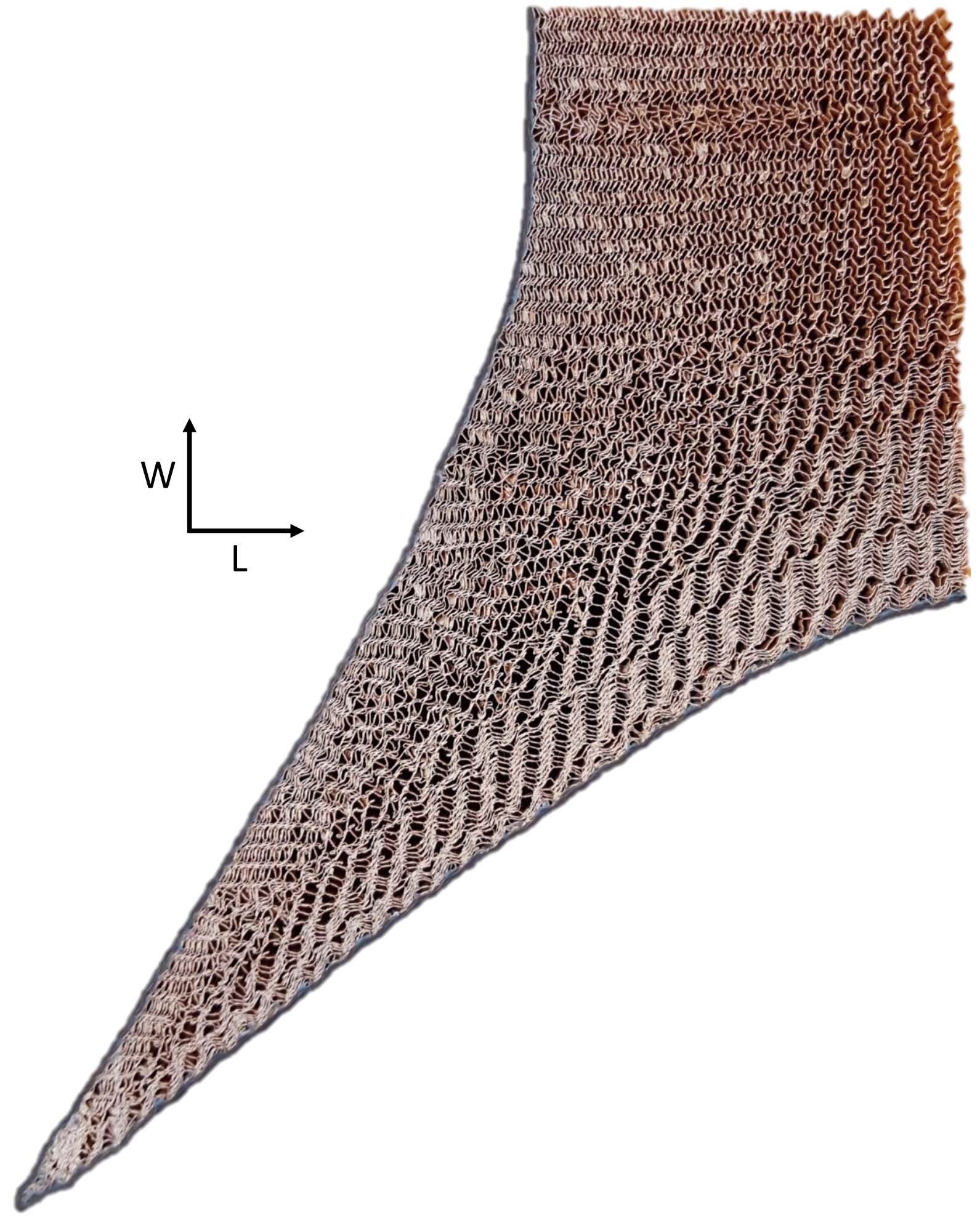

Figure B.31: In-plane section cut of $45^{\circ}$ sample. 


\section{Appendix C}

\section{Appendix C Additional Data Sets}

\section{C.1 $\quad \mathbf{1 2 0}^{\circ} \mathrm{C}_{\text {orig }}$ results}

The following dataset represents the results from the $120^{\circ} \mathrm{C}_{\text {orig }}$ experiment (refer to Section 4.5.2). Unfortunately, LVDT 2 failed during crush resulting in premature flattening out of the $\mathrm{W}$ curve. However, the rate of deformation in $\mathrm{W}$ can be inferred from the original slope of LVDT 2 following collapse. This figure shows a quicker rate of deformation in L compared to $\mathrm{W}$.

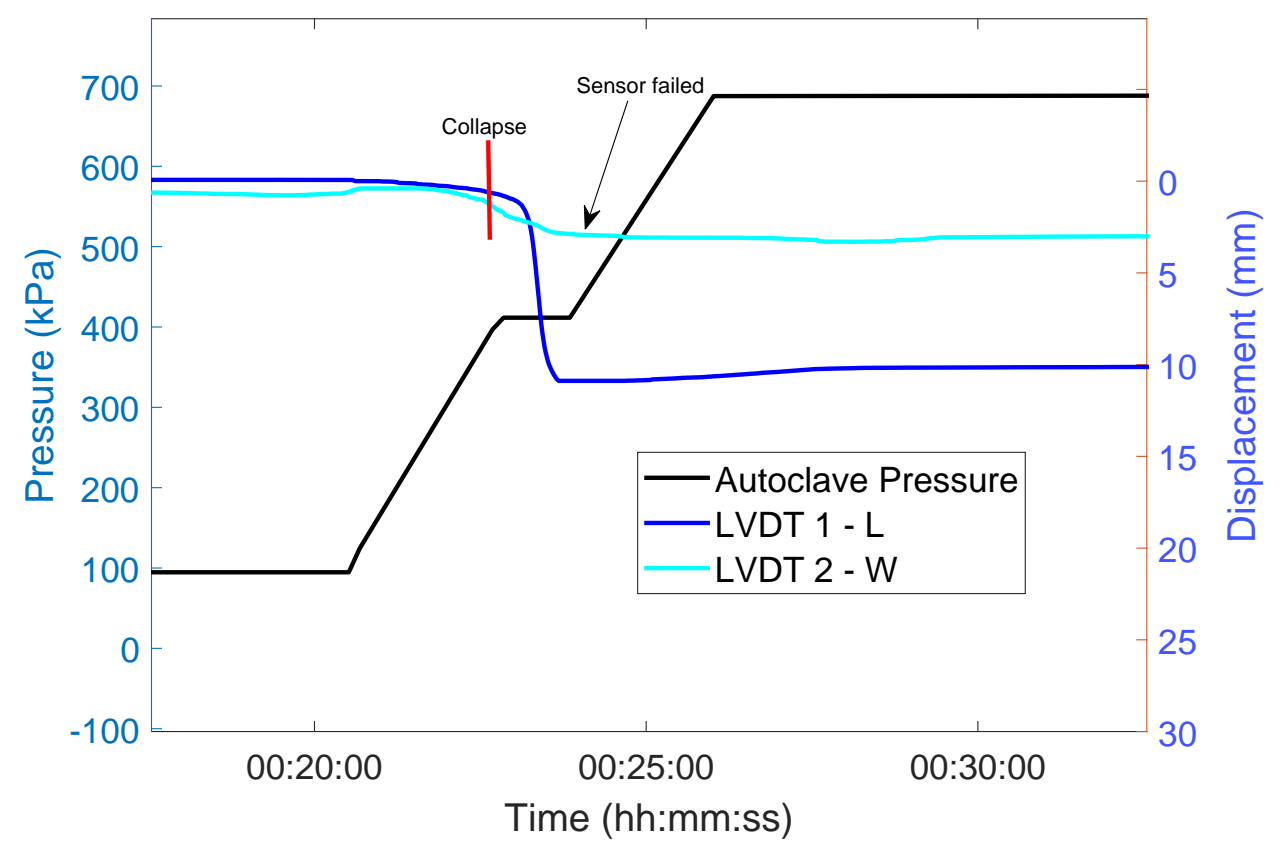

Figure C.1: Core movement in ribbon and non-ribbon direction for $120^{\circ} \mathrm{C}_{\text {orig }}$ sample. Autoclave pressure is absolute external pressure. Vacuum pressure is not shown. 